\title{
Hybrid Organic-Inorganic Solar Cells
}

by

Svetlana Demtchenko, B.Eng.

A thesis submitted to the Faculty of Graduate and Postdoctoral Affairs in partial fulfilment of the requirements for the degree of

Doctor of Philosophy

in

Electrical and Computer Engineering

Ottawa Carleton Institute for

Electrical and Computer Engineering

Department of Electronics

Carleton University

Ottawa, Ontario, Canada

December 2016

Copyright (c) 2016

Svetlana Demtchenko 


\section{Abstract}

This thesis explores the feasibility of a hybrid metal-insulator-semiconductor (MIS) diode with poly(3,4-ethylendioxythiophene) (PEDOT) conductor for photovoltaic (PV) applications and advances the understanding of conductive polymers and their effects on hybrid systems.

In a proof-of-concept experiment it was demonstrated that a photosensitive MIS junction can be created on $n$-type silicon with thermally-grown oxide and a transparent conductive polymer, PEDOT doped with polystyrene sulfonate (PSS). The non-optimized measured devices had an estimated efficiency of $1.65 \%$ under simulated solar illumination. The predicted efficiency for an optimized device was estimated at nearly $12 \%$. A variety of PEDOT formulations were characterized and their suitability for photovoltaic cell fabrication assessed. An in situ polymerized PEDOT dispersed in a silica matrix prepared in a sol-gel process was deemed to be

the best candidate due to its superior adhesion to a $\mathrm{SiO}_{2}$ surface, compatibility with nanostructured surfaces and highly adjustable conductivity and transparency.

For the first time the operation of a hybrid MIS diode with PEDOT:PSS conductor was compared to an inorganic equivalent with gold. For this purpose two sets of devices $\mathrm{Au} / \mathrm{SiO}_{2} / \mathrm{Si}$ and PEDOT:PSS $/ \mathrm{SiO}_{2} / \mathrm{Si}$ were fabricated in parallel and analyzed using a purpose-built, physics-based simulator. Measurement of these devices reveal lower current densities in the hybrid devices as compared to the inorganic ones. It is sometimes proposed in the literature that a formation of a dipole at the 
PEDOT:PSS/silicon interface effectively lowers the work function of the conductive polymer. However, the fitting of the measured data supports the theory of similar work functions of the two conductors. The simulations reveal that the lower current density in the hybrid devices is not due to effective lowering of the work function, but rather due to the limited extent of the energy bands in the conductive polymer, PEDOT:PSS, which cannot be ignored for predicting device performance in photovoltaics as well as organic and hybrid electronics.

The feasibility of nanostructuring the silicon substrate in the MIS device architecture for the purpose of increasing the photovoltaic efficiency was also investigated. The possibility of the effective increase in surface recombination due to surface nanostructuring hindering device performance was assessed. It was determined that the PV efficiency increase due to the enhanced light capture outweighs the surface effects. In the context of a hybrid MIS device, conductive polymer transparency was identified as the factor limiting potential efficiency improvement. A transparency/conductivity optimization scheme is proposed for optimizing the nanostructured hybrid MIS design. 


\section{Acknowledgments}

Had it not been for the support of family, friends, and colleagues, this thesis would not have come to be completed.

To my supervisor, Dr. Steven McGarry, I thank you for sticking by my side through a process that seemed arduous at times.

One exceptionally bright moment during my time as a graduate student was meeting my husband Ryan in the cubicle next door. He taught me that it's OK to be

silly, brought me to tears in laughter, and continues to support me with love and understanding.

To my parents, Dmitri and Galina, thank you for raising me to be stubborn enough to see this undertaking through. Thank you for being so understanding and for always wanting me to be happy.

Carol, Rob, Rick, and Angela, thank you for your expertise and resourcefulness in the fab. You are an indispensable part of research at Carleton.

For many inspirational discussions, thank you Garry and Al. Your kindness and support are greatly appreciated.

Thank you to the administrative staff of the Electronics Department, Blazenka, Sylvie, and Anna, for looking out for all their students. They made the office a home away from home.

Grammar is not my strong suit, "as such" Louise and Dylan's help for editing my thesis was most appreciated. "Thus" I thank you for managing to read all of it. 


\section{Table of Contents}

Abstract $\quad$ ii

Acknowledgments $\quad$ iv

Table of Contents $\quad$ v

List of Tables $\quad$ ix

List of Figures $\quad$ xi

List of Acronyms $\quad$ xxi

List of Symbols $\quad$ xxiv

1 Introduction 1

1.1 Motivation . . . . . . . . . . . . . . . . . . 1

1.2 Objectives . . . . . . . . . . . . . . . . . . . . 3

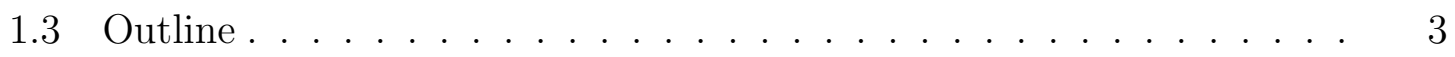

2 Background 5

2.1 Photovoltaics .......................... 5

2.1.1 Figures of Merit . . . . . . . . . . . . 6

2.1.2 Grid Parity . . . . . . . . . . . . . . 12

2.1.3 State of the Industry . . . . . . . . . . . . . . . . . . . . . . . 12 
2.2 MIS Devices . . . . . . . . . . . . . . . . . . . . 13

2.3 Si Nanostructuring . . . . . . . . . . . . . . . . . . . . . . . . . . . . 19

2.4 Insulator Layer . . . . . . . . . . . . . . . . . . . . . . . 23

2.5 Organic Conductors . . . . . . . . . . . . . . . 25

2.6 Transport Models . . . . . . . . . . . . . . . . . . . . . . . . 30

2.6.1 Silicon ........................... 31

2.6.2 Tunneling through Dielectric . . . . . . . . . . . . . . . 32

2.6.3 Polymeric Conductor . . . . . . . . . . . . . . . . . . . . . . 37

2.6 .4 Interface States . . . . . . . . . . . . . . . . . . . . . . 37

2.7 Conclusion . . . . . . . . . . . . . . . . . . . 38

$\begin{array}{lll}3 & \text { PEDOT } & 40\end{array}$

3.1 PEDOT:PSS . . . . . . . . . . . . . . . . . 42

3.1.1 Deposition ....................... 44

3.1.2 Characterization ................. . . . 44

3.1.3 Devices ........................... 45

3.1.4 Compatibility with Nanostructures . . . . . . . . . . 52

3.2 In situ PEDOT . . . . . . . . . . . . . . . . . . . . . 53

3.2.1 Deposition ................... . . 53

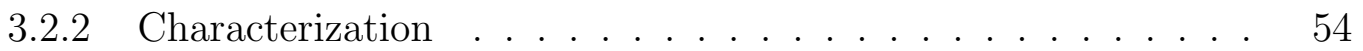

3.2.3 Compatibility with Nanostructures . . . . . . . . . 56

3.3 PEDOT/Silica Composite . . . . . . . . . . . . . . 57

3.3.1 Deposition ........................ 57

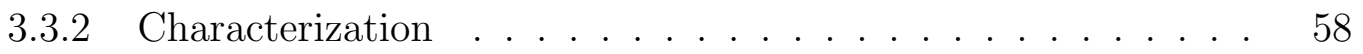

3.3.3 Devices ........................ 60

3.3.4 Compatibility with Nanostructures . . . . . . . . . . . 62

3.4 Conclusion . . . . . . . . . . . . . . . . 63 
$\begin{array}{lll}4 & \text { Nanostructuring } & 67\end{array}$

4.1 Structure Requirements . . . . . . . . . . . . . . . . . 70

4.2 Nanostructuring Techniques . . . . . . . . . . . . . . . 70

4.2.1 RIE silicon grass . . . . . . . . . . . . . . . . 71

4.2.2 Gold-catalized wet etch . . . . . . . . . . . . . . . . . 72

4.3 Atomic Layer Deposition . . . . . . . . . . . . . . . . . . . . . 73

4.4 Problems and Solutions . . . . . . . . . . . . . . . . 76

4.5 Devices . . . . . . . . . . . . . . . . . . . . 79

4.5.1 Fabrication . . . . . . . . . . . . . . . . . 79

4.5.2 Analysis ..................... 80

4.6 Conclusion . . . . . . . . . . . . . . . . . . . . . . 82

5 Semiconductor Solver $\quad 86$

5.1 Physics Covered and Assumptions Made . . . . . . . . . . . . 86

5.2 Methods and Algorithms . . . . . . . . . . . . . . . . . . . . . . 90

5.3 Verification of the Simulator . . . . . . . . . . . . . . 96

$\begin{array}{lll}6 & \text { PEDOT versus } \mathrm{Au} & 98\end{array}$

6.1 Processing . . . . . . . . . . . . . . . . . . . . . . . . . 99

6.2 Comparison . . . . . . . . . . . . . . . . . 100

6.3 Difference in Current Densities . . . . . . . . . . . . . . . . . . 102

6.4 Simulated versus Measured Data . . . . . . . . . . . . . 105

6.4.1 $\mathrm{Au} / \mathrm{SiO}_{2} / \mathrm{Si} \ldots \ldots \ldots . \ldots . \ldots . \ldots 106$

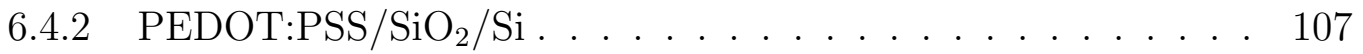

6.5 Conclusion . . . . . . . . . . . . . . . . . . . . 112

7 Conclusions $\quad 113$

7.1 Future Work . . . . . . . . . . . . . . . . . . . . . 115 
7.2 Disseminations . . . . . . . . . . . . . . . . . 116

$\begin{array}{ll}\text { List of References } & 118\end{array}$

Appendix A CV Measurement of an MIS Diode and Work Function Extraction 133

A.1 Work Function Extraction for Diodes with PEDOT:PSS . . . . . . 134

A.2 Work function Extraction for Diodes with PEDOT:PSS/Silica Composite136

Appendix B Computation of Reflectivity for Nanostructured Silicon $\begin{array}{ll}\text { Substrates } & 138\end{array}$

Appendix C PlasmaTherm SLR-772 ECR Etcher Recipe for Black Silicon.

Appendix D Increase in Recombination Current due to Surface Nanostructuring

Appendix E Optimal Resistivities/Transparencies for two Phase filling of Nanostructured Substrates with PEDOT/Silica

Appendix F A Comparative Study of Minimum Power Loss for the Planarized and Structured Devices 


\section{List of Tables}

3.1 Comparison of transparent conductors relevant to choosing the top electrode for a minority-carrier MIS device. . . . . . . . . . . . . . . . 41

3.2 The extracted oxide thicknesses and effective work functions of MIS devices on $n$-type silicon with PEDOT:PSS. . . . . . . . . . . . 46

3.3 Substrate doping densities measured with a four-point probe as well as extracted from the CV measurements of the hybrid diodes made with

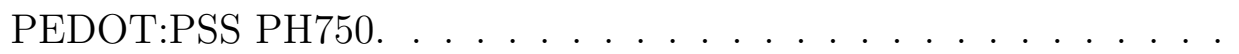

3.4 Ratios by weight of sol-gel and PEDOT mixtures for the composite as well as ratios by weight of various constituents of the in situ polymerization mixture for PEDOT. . . . . . . . . . . . . . . . . 59

3.5 Conductivities and thicknesses of various PEDOT/silica samples. . . 59

5.1 Parameters used in device simulations. . . . . . . . . . . . . . . . . 91

A.1 Quantities involved in extracting the conductor work function of MIS diodes from the CV characteristics. . . . . . . . . . . . . 135

A.2 The relevant quantities for extraction the work function of the conductor from the CV characteristics of a Schottky junction. The resistivity of the substrate was taken to be $1.5 \Omega \mathrm{cm}$ for these calculations. This assumption adds an uncertainty of only $0.01 \mathrm{eV}$ to the extracted work function value. . . . . . . . . . . . . . . . . . 
B.1 Parameters of the harmonic oscillator model for the permittivity of silicon when the wavelength is expressed in nm. . . . . . . . . . . 139

C.1 PlasmaTherm SLR-772 ECR etcher recipe for black silicon. . . . . . 143 


\section{List of Figures}

2.1 Representation of a metal-semiconductor (Schottky) diode solar cell (a) pictorial (b) band structure. The incident light is absorbed by the substrate, generating an electron-hole pair. The electric field in the depletion region pulls the charges apart and sweeps them to the contacts for collection. . . . . . . . . . . . . . . . .

2.2 Dependence of the maximum single junction solar cell efficiency on the material bandgap. The actual device efficiency is likely to be lower due to various optical and electrical losses. Efficiencies for irradiation at the Earth's surface (AM1.5) are shown. . . . . . . . . . . . .

2.3 Bandstructures and absorption coefficients for direct and indirect semiconductors. . . . . . . . . . . . . . . .

2.4 Dark and illuminated IV characteristics for a solar cell with $I_{s c}, V_{o c}$ and $F F$ indicated. . . . . . . . . . . . . . . . . . . . . . . 11

2.5 The 2016 NREL survey of the state of the photovoltaic industry. . . . 14

2.6 Band diagram of an MIS device with various relevant variables labelled. 15

2.7 Energy-band diagram at the surface of an $n$-type semiconductor. The reference for the potential energy is taken to be the intrinsic Fermi level in the bulk of Si. Weak inversion occurs when the surface potential, $\phi_{S}$, exceeds $\phi_{B}$ as defined in this figure. Strong inversion is said to occur for $\phi_{S}>2 \phi_{B}$. Illustration based on Sze. . . . . . . . . . . . . 17 
2.8 Band diagram of an MIS device showing possible current paths (1) metal - conduction band tunneling (2) metal - valence band tunneling (3) metal - surface states tunneling (4) surface states - conduction/valence band recombination (5) drift - diffusion. . . . . . . . . . . . 18

2.9 Sketch of a nanostructured surface with relevant variables labelled. . 20

2.10 Effect of insulator layer thickness on the parameters of a photovoltaic

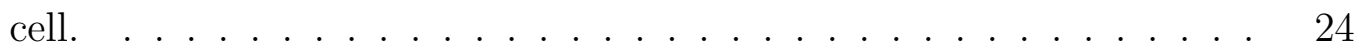

2.11 Illustration of an ALD process. . . . . . . . . . . . . . 26

2.12 Conduction in polymers. (a) Formation of thin energy bands in polymeric semiconductors. The thickness of the bands depends on the degree of interaction between the molecules in the solid. (b) Inter-chain hopping. . . . . . . . . . . . . . . . 27

2.13 Band diagrams of polymeric semiconductors in various states of doping. 28

2.14 Chemical structure of PEDOT:PSS. . . . . . . . . . . . . . . 28

2.15 Band diagram of an MIS devise showing possible current paths. $J_{T C}$ : metal - conduction band tunneling; $J_{T V}$ : metal - valence band tunneling; $J_{T S}$ : metal - surface states tunneling; $J_{R C}$ : surface states conduction band recombination; $J_{R V}$ : surface states - valence band recombination; $J_{n} / J_{p}$ : drift - diffusion. . . . . . . . . . . .

2.16 Tunneling current paths in (a) conventional MIS device (b) MIS device with a polymeric conductor. . . . . . . . . . . . . .

3.1 Transmission of $340 \mathrm{~nm}$ of dry Clevios PH750 PEDOT:PSS film relative to a clean glass substrate. . . . . . . . . . . . . . 45

3.2 Desired structure for experimental hybrid MIS diodes. . . . . . . . . . 46 
3.3 Capacitance-voltage (CV) characteristics of MIS devices on $n$-type silicon with thermally-grown oxide and PEDOT:PSS conductor. The measurements are normalized to the active device area extracted from the CV measurements. The inset zooms in on the voltage intercepts of the presented linear fits, from which the PEDOT:PSS work function is calculated. . . . . . . . . . . . . . . .

3.4 Current-voltage (IV) characteristics of MIS devices on $n$-type silicon with thermally-grown oxide and PEDOT:PSS conductor. (a) Dark reverse bias current (b) Dark forward bias current (c) Dark currentvoltage characteristic on a logarithmic scale with the curves offset from one another for better visibility (d) Photoresponce under halogen light illumination. . . . . . . . . . . . . . . . .

3.5 Explanation given by Card and Rhoderick for a higher reverse bias current density over a range of biases for an MIS diode with a thicker tunneling oxide (a) Card's data for two MIS diodes with different oxide thicknesses (b) thick oxide interfacial layer allows the metal Fermi level to better align with the conduction band; higher availability of electrons in the metal at the band edge overcompensates for the thicker tunneling barrier (c) thin oxide does not support as much of a voltage drop across it and the metal Fermi level cannot pull up to the conduction band edge. . . . . . . . . . . . . . . .

3.6 Current-voltage characteristics of a flat diode on $n$-type silicon with no intentional interfacial oxide and PEDOT:PSS conductor. The measurements are performed in a solar simulator with 0.8 sun illumination. 52 
3.7 Scanning electron microscope (SEM) image of reactive ion etched silicon grass (a) as fabricated (b) with vacuum-deposited Clevios PH750. The details of the deposition process are explained in Chapter 4. The scale bar is $1 \mu \mathrm{m} . \ldots \ldots \ldots \ldots \ldots \ldots \ldots \ldots \ldots$

3.8 Transmission spectrum of $306 \mathrm{~nm}$ thick in situ PEDOT. . . . . . . . 55

3.9 SEM image of reactive-ion-etched silicon grass with in situ deposited PEDOT . . . . . . . . . . . . . . . . . . . .

3.10 Transmission spectrum of PEDOT/silica composites. The irregularities in the spectrum at $380 \mathrm{~nm}, 560 \mathrm{~nm}, 690 \mathrm{~nm}$, and $810 \mathrm{~nm}$ are measurement artifacts.

3.11 CV characteristics of Schottky junctions made with $n$-type silicon (1-2 $\Omega \mathrm{cm}$ ) and PEDOT/silica composites of slightly different compositions. The areas of the devices are different. The resistivity of the substrate was taken to be $1.5 \Omega \mathrm{cm}$ for these calculations. This assumption adds an uncertainty of only $0.01 \mathrm{eV}$ to the extracted work function value. . 61

3.12 Sample D in the dark and under halogen lamp illumination. . . . . . 62

3.13 SEM image of reactive-ion-etched silicon grass with deposited PEDOT/silica composite. . . . . . . . . . . . . . .

3.14 Electrical (a) and optical (b) properties of the PEDOT/silica composites showing increasing conductivity and optical loss with increasing PEDOT content. . . . . . . . . . . . . . . . . . . .

3.15 Sketch of a nanostructured solar cell that uses two types of PEDOT: one for best optical transparency without compromising the transition of charge up to the top of the nanostructure and another, more conductive, thin layer for horizontal transmission of charge to the finger grid electrodes for collection. . . . . . . . . . . . . . . . . . . 
3.16 Sketch of a nanostructured solar cell with a highly conductive conformal layer of PEDOT. The spacing of the finger grid is limited by the conductivity of PEDOT. . . . . . . . . . . . . . .

4.1 Absorption coefficient of silicon. The light at lower wavelengths (higher energies) is absorbed more readily then at higher wavelengths (lower energies). . . . . . . . . . . . . . . .

4.2 (a) SEM image of pyramidal structure in silicon created with an anisotropic alkaline etch (b) Reflectivity of such a structure with the reflectivity of polished silicon surface shown for reference. . . . . . . .

4.3 Simulated reflectivity of a nanostructured surface in air with various feature sizes and heights (a) approximation to the nanostructure geometry used in the numerical calculations (b) resultant reflectivities. . 71

4.4 Formation of silicon grass by reactive ion etching in $\mathrm{SF}_{6} / \mathrm{O}_{2}$ environment. 72

4.5 RIE silicon grass nanostructuring and corresponding reflection spectrum; (a) SEM image of RIE silicon grass (b) reflectivity of silicon grass at a single angle of $20^{\circ}$ measured with a Lamda900 spectrometer. 73

4.6 Gold-assisted chemical wet etch of silicon (a) process illustration (b) SEM image of the result. . . . . . . . . . . . . . . . . . . 74

4.7 Illustration of the ALD process. First precursor is adsorbed to the surface until a complete monolayer is formed, then evacuated with a purging gas. The second precursor reacts with the new surface until a complete monolayer of the desired material in produced, $\mathrm{Al}_{2} \mathrm{O}_{3}$ in this case. The rest of the precursor is, again, purged. The cycle is repeated until the desired thickness is reached. . . . . . . . . . . . . . . . 75

4.8 Effective surface area increase due to nanostructuring for reflection minimization. . . . . . . . . . . . . . . . . 77 
4.9 Filling of RIE Si nanostructure with a PEDOT/silica composite. (a) Composite was spin cast and dried on the hotplate in air. (b) Composite was deposited on the substrate and dried in vacuum. The scale

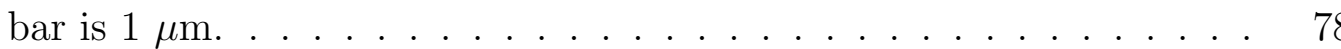

4.10 PEDOT deposition schemes for solving the PEDOT shading problem (a) two types of PEDOT: one for best optical transparency without compromising the transition of charge up to the top of the nanostructure and another, more conductive, thin layer for horizontal transmission of charge to the finger grid electrodes for collection. (b) thin highly conductive conformal layer of PEDOT; the spacing of the finger grid is limited by the conductivity of PEDOT. . . . . . . . . . . .

4.11 Current-voltage characteristics of the two best performing solar cells constructed with $\mathrm{Al}_{2} \mathrm{O}_{3}$ insulator on RIE silicon substrate in the dark and under halogen lamp illumination. The recorded efficiencies are $1.6 \% \pm 0.4 \%$ and $1.7 \% \pm 0.4 \% \ldots \ldots \ldots$

4.12 Measured efficiencies of MIS solar cells fabricated on RIE etched $n$ type silicon substrates with $\mathrm{ALD}$ deposited $\mathrm{Al}_{2} \mathrm{O}_{3}$ insulator and in situ PEDOT (a) table of calculated efficiencies (b) average measured efficiencies as a function of dielectric thickness. . . . . . . . . . .

4.13 Relative power conversion efficiencies of flat versus nanostructured MIS diodes with ALD deposited $\mathrm{Al}_{2} \mathrm{O}_{3}$ insulator and PEDOT:PSS conductor. The error on the efficiency shown is mostly due to the uncertainty in the incident light intensity, as the irradiation varies widely with lateral lamp alignment as well as its distance away from the sample. .

5.1 Algorithm used by the simulator to solve the continuity equation. Modifications included in the model for PEDOT are highlighted in gray. 
5.3 Comparison of simulated and calculated current-voltage characteristics of Schottky diodes (a) $N_{D}=5 \times 10^{14} \mathrm{~cm}^{-3}, \phi_{m}=4.9 \mathrm{eV}$ (b) $N_{D}=5 \times 10^{15} \mathrm{~cm}^{-3}, \phi_{m}=4.8 \mathrm{eV} \ldots \ldots \ldots \ldots$

6.1 Samples of top conductor patterning for hybrid and inorganic diodes. The light blue window is the opening in the isolating $100 \mathrm{~nm}$ oxide, where only a thin tunneling oxide is present. The materials are pattered such that there is no overlap with the thick oxide. The markings in the middle of the pattered squares are spots where the probe was landed.(a) Example of scratch-patterned PEDOT. (b) Example of pattered Au. . 100

6.2 Average dark IV characteristics of several inorganic $\mathrm{Au} / \mathrm{SiO}_{2} / \mathrm{Si}$ and hybrid PEDOT:PSS/SiO $/ 2 / \mathrm{Si}$ diodes. The error bars represent the standard deviations in the measured current values of several identical

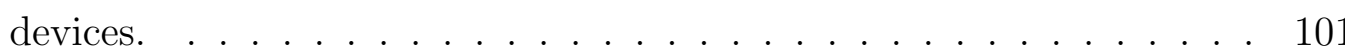

6.3 Energy band diagrams for (a) an inorganic $\mathrm{Au} / \mathrm{SiO}_{2} / \mathrm{Si}$ diode (b) a hybrid PEDOT:PSS/SiO $2 / \mathrm{Si}$ diode. The thin energy bands of the conductive polymer may limit the current density in the hybrid diode. No such limitation exists in the inorganic device. . . . . . . . . . . . . 103

6.4 Density of states (DOS) for materials of different dimensionality. . . . 104

6.5 Density of states used for PEDOT in simulation. . . . . . . . . . . . 105

6.6 Origin of PEDOT band structure (a) PEDOT:PSS absorption spectrum (b) possible PEDOT:PSS band structure with various doping levels. . . . . . . . . . . . . . . . . . . 106

6.7 Simulated fit for the measured data of a $\mathrm{Au} / \mathrm{SiO}_{2} / \mathrm{Si} \mathrm{MIS}$ diode. The simulation parameters used are $N_{D}=6.71 \times 10^{14} \mathrm{~cm}^{-3}, \phi_{m}=4.8 \mathrm{eV}$, $t_{o x}=1.63 \mathrm{~nm}$, and electron and hole effective masses in oxide of $0.4 \mathrm{~m}_{e}$ and $0.3 \mathrm{~m}_{e}$ respectively. The distribution of surface states used in this simulation is shown in Figure 6.8. . . . . . . . . . . . . . . 108 
6.8 Interface state density at the $\mathrm{Si} / \mathrm{SiO}_{2}$ interface as defined by the DIGS model. The left-hand y-axis corresponds to the surface state profile that gave the best fit for $\mathrm{Au} / \mathrm{SiO}_{2} / \mathrm{Si}$ diodes. The right-hand $\mathrm{y}$ axis corresponds to the surface state profile that gave the best fit for PEDOT:PSS/SiO $/ \mathrm{Si}_{2} / \mathrm{Si}$ diodes. . . . . . . . . . . . . . . 108

6.9 Measured and simulated current voltage characteristics of a typical PEDOT-based MIS diode. The simulation parameters used are $N_{D}=6.95 \times 10^{14} \mathrm{~cm}^{-3}, \phi_{m}=4.76 \mathrm{eV}, t_{o x}=1.93 \mathrm{~nm}$, and electron and hole effective masses in oxide of $0.4 \mathrm{~m}_{e}$ and $0.3 \mathrm{~m}_{e}$ respectively. The distribution of surface states used in this simulation is shown in Figure

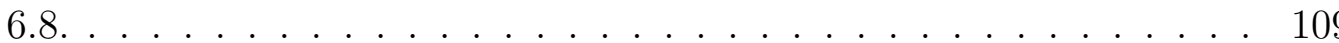

6.10 Simulated PEDOT:PSS bands at $0 \mathrm{~V}$ applied bias. The density of states is adjusted such that the carrier density of $N_{\text {holes }}=$ $3 \times 10^{20} \mathrm{~cm}^{-3}$. The red lines indicate the edges of the conduction and valence bands of the silicon substrate. The black line indicates the position of the Fermi level. . . . . . . . . . . . . . . . .

A.1 CV characteristics of several MIS devices with PEDOT:PSS PH750. The device areas vary. The labels indicate the oxidation temperature for interfacial oxide growth and the least squares linear fit to the data. 135

A.2 CV characteristics of Schottky junctions made with $n$-type silicon (1$2 \Omega \mathrm{cm})$ and PEDOT/silica composites of slightly different compositions. The areas of the devices are different. . . . . . . . . . . . . 136

B.1 Numerical fit for the complex permittivity of silicon modelled as a sum of two harmonic oscillators (a) real part of the relative permittivity (b) imaginary part of the relative permittivity. . . . . . . . . . . . . . . 139

D.1 Effective surface area increase due to nanostructuring for reflection

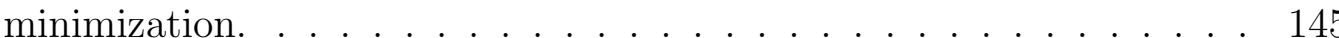


E.1 Sketch of a nanostructured solar cell that uses two types of PEDOT: one for best optical transparency without compromising the transition of charge up to the top of the nanostructure and another, more conductive, thin layer for horizontal transmission of charge to the finger grid electrodes for charge collection. . . . . . . . . . . . . . . . . . . 147

E.2 Geometry for estimating PEDOT resistivity. . . . . . . . . . . . . . . 148

E.3 Properties of PEDOT/silica composites as reported by Lee (blue); results obtained in this work (sample IDs are defined in Chapter 3) are included for reference but were not used to calculate the best fits (red) (a) conductivity vs PEDOT content for composites with EDOT/FTS $=1 / 2.25 \mathrm{~mol}$ ratio $(\mathrm{b})$ transmittance vs PEDOT content for composited with $\mathrm{EDOT} / \mathrm{FTS}=1 / 2.25 \mathrm{~mol}$ ratio $(\mathrm{c})$ transmittance $v$ PEDOT thickness for composites with PEDOT ratio of 0.8 . . . . . . . . . . 151

E.4 Power loss optimization for PEDOT/silica filled nanostructure $1000 \mathrm{~nm}$ high based on the composite parameters of Lee et al. . . . . . . . . . 152

F.1 PEDOT deposition schemes for solving the PEDOT shading problem (a) two types of PEDOT: one for best optical transparency without compromising the transition of charge up to the top of the nanostructure and another, more conductive, thin layer for horizontal transmission of charge to the finger grid electrodes for collection. (b) thin highly conductive conformal layer of PEDOT; the spacing of the finger grid is limited by the conductivity of PEDOT. . . . . . . . . . . . . . . . 154

F.2 Series of optical and resistive loss curves as function of finger grid spacing for various PEDOT thicknesses. The minimum power loss for each PEDOT thickness is indicated with an "o". . . . . . . . . . . . . 155 
F.3 Surface structure parameters for minimizing power loss with $100 \mu \mathrm{m}$ wide current collection fingers and $1485 \mathrm{~S} / \mathrm{cm}$ PEDOT. (a) minimum power loss achieved with optimal finger spacing (b) optimal finger spacing for minimizing power loss. . . . . . . . . . . . . . . . . 156

F.4 Simulated reflectivities of a triangular silicon structures $1 \mu \mathrm{m}$ deep. One is empty and the other is filled with PEDOT. . . . . . . . . . 157 


\section{List of Acronyms}

\begin{tabular}{|c|c|}
\hline Acronym & Definition \\
\hline AFM & Atomic Force Microscopy \\
\hline ALD & Atomic Layer Deposition \\
\hline $\mathrm{AM}$ & Air Mass \\
\hline $\mathrm{BP}$ & Bipolaron \\
\hline CIGS & Cadminum Indium Galium Selenide \\
\hline $\mathrm{CV}$ & Capacitance Voltage \\
\hline CVD & Chemical Vapour Deposition \\
\hline $\mathrm{CZ}$ & Czochralski \\
\hline DI & Deionized \\
\hline DMSO & Dimethyl Sulfoxide \\
\hline DOS & Density of States \\
\hline EDOT & 3,4-ethylenedioxythiophene \\
\hline EDS & Energy-dispersive X-Ray Spectroscopy \\
\hline
\end{tabular}




\begin{tabular}{|c|c|}
\hline $\mathrm{FF}$ & Fill Factor \\
\hline FTS & Fe(III)-tosylate \\
\hline GLAD & Glancing Angle Deposition \\
\hline $\mathrm{HF}$ & Hydrofluoric Acid \\
\hline HMDS & Hexamethyldisilazane \\
\hline HOMO & Highest Occupied Molecular Orbital \\
\hline IPA & Isopropanol \\
\hline ITO & Indium Tin Oxide \\
\hline IV & Current Voltage \\
\hline LUMO & Lowest Unoccupied Molecular Orbital \\
\hline MIS & Metal Insulator Semiconductor \\
\hline MOS & Metal Oxide Semiconductor \\
\hline MOSFET & Metal Oxide Semiconductor Field Effect Transistor \\
\hline PANI & Polyaniline \\
\hline PEDOT & Poly(3,4-ethylenedioxythiophene) \\
\hline PSS & Polystyrene sulfonate \\
\hline PV & Photovoltaic \\
\hline RIE & Reactive Ion Etch \\
\hline $\mathrm{RPM}$ & Revolutions Per Minute \\
\hline
\end{tabular}


SEM Scanning Electron Microscope

SRH Schokley-Read-Hall

TCO Transparent Conductive Oxide

TEOS Tetraethyl orthosilicate

TMAH Tetramethylammonium Hydroxide

UPS Ultraviolet Photoelectron Spectroscopy

VPP Vapour Phase Polymerized 


\section{List of Symbols}

\section{Symbols Definition}

\begin{tabular}{ll}
\hline \hline$A$ & Area \\
$A^{* *}$ & Richardson constant \\
$\alpha$ & Attenuation coefficient \\
$c$ & Speed of light \\
$C_{D}$ & Depletion capacitance \\
$C_{o x}$ & Oxide capacitance \\
$\chi_{S i}$ & Electron affinity of silicon \\
$\chi_{o x}$ & Electron affinity of silicon dioxide \\
$D_{i t}$ & Interface state density \\
$D_{n}$ & Diffusion constant for electrons \\
$D_{p}$ & Diffusion constant for holes \\
$E$ & Electric field or Energy \\
$E_{B P_{1}}$ & Energy level of the anti-bonding bipolaron band \\
& \\
&
\end{tabular}




\begin{tabular}{|c|c|}
\hline$E_{B P_{2}}$ & Energy level of bonding bipolaron band \\
\hline$E_{C}$ & Conduction band energy level \\
\hline$E_{f n}$ & Quasi-Fermi level for electrons \\
\hline$E_{f p}$ & Quasi-Fermi level for holes \\
\hline$E_{G}$ & Bandgap Energy \\
\hline$E_{\text {НОМО }}$ & Energy level of the highest occupied molecular orbital \\
\hline$E_{L U M O}$ & Energy level of the lowest unoccupied molecular orbital \\
\hline$E_{T}$ & Trap energy level \\
\hline$E_{V}$ & Valence band energy level \\
\hline$\eta$ & Efficiency \\
\hline$\epsilon$ & Permittivity \\
\hline$f_{s s}$ & Occupational probability for electrons in the surface states \\
\hline$f_{m}$ & Occupational probability for electrons in the top electrode \\
\hline$f_{s}$ & Occupational probability for electrons in the substrate \\
\hline$f_{m}^{\prime}$ & Occupational probability for holes in the top electrode \\
\hline$f_{s}^{\prime}$ & Occupational probability for holes in the top substrate \\
\hline$F F$ & Fill factor \\
\hline$G$ & Generation rate \\
\hline$h$ & Planck's constant \\
\hline
\end{tabular}




\begin{tabular}{|c|c|}
\hline$\hbar$ & $h / 2 \pi$ \\
\hline$I$ & Current \\
\hline$I_{o}$ & Reverse saturation current \\
\hline$I_{s c}$ & Short circuit current \\
\hline$J$ & Current density \\
\hline$J_{g}$ & Generation current density \\
\hline$J_{n}$ & Electron current density \\
\hline$J_{p}$ & Hole current density \\
\hline$J_{r}$ & Recombination current density \\
\hline$J_{R C}$ & $\begin{array}{l}\text { Generation/recombination current density between con- } \\
\text { duction band and surface states }\end{array}$ \\
\hline$J_{R V}$ & $\begin{array}{l}\text { Generation/recombination current density between valence } \\
\text { band and surface states }\end{array}$ \\
\hline$J_{T C}$ & Conduction band tunneling current density \\
\hline$J_{T S}$ & Tunneling current density between metal and surface states \\
\hline$J_{T V}$ & Valence band tunneling current density \\
\hline$k_{B}$ or $k$ & Boltzmann's consant \\
\hline$k_{i}$ & Wave vector of charge carriers in the insulator \\
\hline$k_{m}$ & Wave vector of charge carriers in the metal \\
\hline
\end{tabular}


$k_{s} \quad$ Wave vector of charge carriers in the semiconductor

$L_{D} \quad$ Debye length

$m_{d e}^{*} \quad$ Density of states effective mass of an electron

$m_{d h}^{*} \quad$ Density of states effective mass of a hole

$m_{e}^{*} \quad$ Effective mass of an electron

$m_{h}^{*} \quad$ Effective mass of a hole

$m_{i} \quad$ Effective mass of charge carriers in the insulator

$m_{m} \quad$ Effective mass of charge carriers in the metal

$m_{s} \quad$ Effective mass of charge carriers in the semiconductor

$\mu_{n} \quad$ Electron mobility

$\mu_{p} \quad$ Hole mobility

$n \quad$ Diode ideality constant or electron concentration

$N_{A} \quad$ Acceptor doping density

$N_{D} \quad$ Donor doping density

$n_{i} \quad$ Intrinsic carrier density

$N_{n} \quad$ Density of state for electrons

$N_{p} \quad$ Density of state for holes

$N_{p o l y, n} \quad$ Density of state for electrons in the polymer

$N_{\text {poly }, p} \quad$ Density of state for holes in the polymer 


\begin{tabular}{|c|c|}
\hline$n_{s}$ & Donor concentration at the surface \\
\hline$N_{s s}$ & Surface state density \\
\hline$p$ & Hole concentration \\
\hline$p_{g}$ & Carrier density at surface by illumination \\
\hline$P_{\text {optical }}$ & Optical power \\
\hline$p_{s}$ & Acceptor concentration at the surface \\
\hline$\phi_{B}$ & Potential barrier of a Schottky diode \\
\hline$\phi_{m}$ & Metal work function \\
\hline$\phi_{n}$ & Difference between conduction band edge and Fermi level \\
\hline$\phi_{o x}$ & Potential drop across the oxide \\
\hline$\phi_{s}$ & Surface potential \\
\hline$\psi_{B i}$ & Built-in voltage \\
\hline$q$ & Electronic charge \\
\hline$R_{C}$ & Contact resistance \\
\hline$R_{S}$ & Series resistance \\
\hline$\rho$ & Resistivity \\
\hline$S$ & Surface recombination velocity \\
\hline$S_{\text {eff }}$ & Effective surface recombination velocity \\
\hline$S_{\text {solar }}$ & Solar spectrum \\
\hline
\end{tabular}




\begin{tabular}{|c|c|}
\hline$s_{w}$ & Finger spacing \\
\hline$\sigma$ & Capture cross section or conductivity \\
\hline$T$ & Temperature \\
\hline$T_{q}$ & Quantum tunneling coefficient \\
\hline$T_{o p t}$ & Optical transmission \\
\hline$t_{o x}$ & Oxide thickness \\
\hline$\tau$ & Capture and release time constant of surface states \\
\hline$\tau_{n}$ & Electron lifetime \\
\hline$\tau_{p}$ & Hole lifetime \\
\hline$\tau_{r}$ & Recombination time constant of surface states \\
\hline$\tau_{T}$ & Tunneling time constant of surface states \\
\hline$U$ & Recombination rate \\
\hline$U_{s}$ & Surface recombination rate \\
\hline$V$ & Voltage \\
\hline$V_{o c}$ & Open circuit voltage \\
\hline$v$ & Velocity \\
\hline$v_{n}$ & Electron velocity \\
\hline$v_{p}$ & Hole velocity \\
\hline$v_{t h}$ & Thermal velocity \\
\hline
\end{tabular}

xxix 
W Depletion region width

$w_{f} \quad$ Finger width

$\xi \quad$ Ratio between PEDOT and sol-gel silica by weight 


\section{Chapter 1}

\section{Introduction}

The work described in this thesis was performed to study the fabrication and operation of hybrid metal-insulator-semiconductor diodes for photovoltaic applications. In these devices, an organic polymeric conductor was used instead of a metal in conjunction with conventional dielectrics and silicon semiconductor. At the beginning of this research project no reports of such devices were found. To advance the state of the art in understanding of conductive polymers and their effects on the operation of hybrid devices, an experimental and theoretical comparison of inorganic and hybrid MIS diodes was carried out in this work for the first time. Nanostructuring the substrate surface for enhanced photovoltaic efficiency of hybrid MIS solar cells was also explored as a logical extension of this work, as such structures were known to be explored for conventional inorganic solar cells.

\subsection{Motivation}

In this time of transition from depleting and polluting conventional fuels such as coal and petroleum, solar energy conversion is becoming a staple in renewable green energy portfolios around the world $[1,2]$. With a quarter of all energy consumed being in

the form of electrical power [2], the photovoltaic conversion of optical power directly 
into electricity is a very attractive option.

In the past, the prohibitive costs of materials and fabrication kept solar cells reserved for space and niche consumer applications. However, with the cost advantages of large scale production of electrical grade silicon and government sponsored subsidies, solar cells are finally breaking into the consumer market for power generation. At the same time a variety of new materials and device architectures are being developed to bring the costs down even further.

For decades the field of photovoltaic research was divided into two camps: the quest for higher efficiency inorganic solar cells and the search for cost-saving materials and architectures of organic solar cells. The inorganic materials confer stability, fast charge transfer, and high carrier mobility needed for high-efficiency devices. However, the high temperatures and costly equipment needed for their processing drive up the cost. The organic materials, on the other hand, provide cost-effective, lowtemperature processing techniques, but suffer from high carrier recombination rates and low mobilities. The material degradation is also a concern.

Recently, a new direction in research has emerged, aiming to combine the advantages of both inorganic and organic materials: hybrid solar cells. Currently, most hybrid designs are based on a dispersion of inorganic nanoparticles in an organic matrix. By virtue of particle size, the effective bandgap of the inorganic inclusions is adjusted. Controlling the particle size allows for a tunable absorption profile. Additionally, the inorganics provide faster charge dissociation. As a result, such device architectures should be able to improve upon purely organic devices [3]. Several research groups are reviving the metal-insulator-semiconductor (MIS) diodes for photovoltaic applications. This design garnered much attention in the 1970s and 1980s but was abandoned primarily due to the non-transparent nature of the top metal layer, which limited device efficiency. With the variety of transparent organic conductors now available, the MIS architecture is once again being studied in the field of 
photovoltaics. Despite a number of groups producing and characterizing such devices, the effects of replacing the conventional metal with an organic conductor are not well understood.

\subsection{Objectives}

Improving the understanding of the electrical structure of organic conductors and their effects on device performance in an MIS architecture as well as exploring the possibilities and limitations of such structures for photovoltaic conversion were the primary goals of this work.

To this end, a variety of formulations of a conductive polymer poly(3,4ethylenedioxythiophene) (PEDOT) were investigated. The operation of PEDOTcontaining MIS diodes was compared to the performance of gold-topped MIS diodes fabricated in parallel. A semiconductor solver program was written in MATLAB to analyse the effects of PEDOT band structure and charge transport properties.

Nanostructuring the substrate surface was also explored as means of increasing the solar cell efficiency in an organic hybrid MIS architecture. The increase in efficiency is expected to come from lowering the reflection losses and increasing light absorption at higher wavelengths due to the increase in the optical path length.

\subsection{Outline}

Chapter 2 provides the background on photovoltaics, explaining the general operation of such devices, as well as the figures of merit used in their evaluation and provides the review of the state of the industry. Chapter 3 describes the various formulations of PEDOT studied in this work and the organic hybrid MIS diodes, fabricated with them. Chapter 4 discusses the nanostructuring of the device surface for improved 
operation efficiency. Chapter 5 elaborates the charge transport models used in simulating the device behaviour. Chapter 6 explores the organic conductors as a substitute for the conventional metal in an MIS device. Chapter 7 summarises the completed work and provides recommendations for possible directions in future research. 


\section{Chapter 2}

\section{Background}

\subsection{Photovoltaics}

A photovoltaic process is an elegant, one-step conversion mechanism of optical energy into electricity. This process requires a photon of a suitable energy to be absorbed by a material promoting electrons from their bound states to higher energy levels at which they can conduct electricity. Thus generated electron-hole pairs ${ }^{1}$ need to be separated before they recombine and must also be guided to corresponding electrodes for collection as shown in Figure 2.1. This is the essence of any solar cell. Therefore, a solar cell structure requires a semiconducting material for photoelectric conversion, and a built-in electric field for charge separation. A diode structure satisfies both of these requirements. The bandgap of the chosen semiconductor will dictate the lower limit on the photon energies that can be absorbed by the substrate. Doping density of the substrate determines the extent of the built-in field, termed the depletion region. Lower doping density results in larger depletion region and more efficient charge separation and collection.

\footnotetext{
${ }^{1}$ In the case of highly spatially-confined systems excitons are generated instead. They are tightly bound electron-hole pairs that travel through the material as a unit. In many organic semiconductors polarons are generated instead, which are charges entangled with the bond structure of the organic material. As a polaron moves through a material, the structure of the bonds in the molecule/polymer distorts to accommodate this movement.
} 


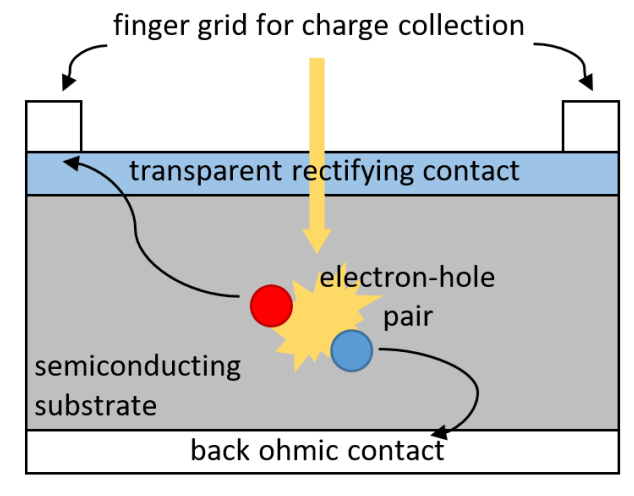

(a)

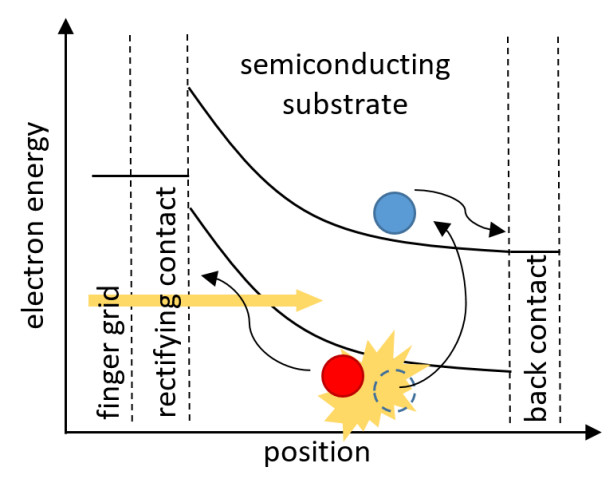

(b)

Figure 2.1: Representation of a metal-semiconductor (Schottky) diode solar cell (a) pictorial (b) band structure. The incident light is absorbed by the substrate, generating an electron-hole pair. The electric field in the depletion region pulls the charges apart and sweeps them to the contacts for collection.

\subsubsection{Figures of Merit}

Solar irradiance varies depending on location, time of day, and weather. This necessitates a clearly defined standard for solar cell testing. The present standard is the solar spectrum on a clear day with the sun at an angle of elevation of $42^{\circ}$, termed AM1.5. This spectrum corresponds to a total irradiance of approximately $970 \mathrm{~W} / \mathrm{m}^{2}$, but is usually normalized to $1000 \mathrm{~W} / \mathrm{m}^{2}$ [4-6]. However, not all this energy can be used. The amount of solar energy that can be converted to electricity by a photovoltaic device is quantified by the efficiency of the device, defined as:

$$
\eta=\frac{P_{\text {electrical-out }}}{P_{\text {optical }- \text { in }}}
$$

Some of the limiting factors for the efficiency of a photovoltaic device are the bandgap of the light absorber used, the reflectivity of the top surface of the cell, and carrier recombination in the bulk and at the interfaces.

The bandgap of the light absorber defines what portion of the solar spectrum can 
be converted to electricity. Light with energies below the bandgap cannot be absorbed and is, therefore, lost. The light with the energies at and above the bandgap can be absorbed, but a portion of the energy above the bandgap is lost to thermalization and is not converted to electricity either. Thus, the efficiency of a single junction solar cell is a function of the bandgap of the light absorber. Too large of a bandgap will lead to large absorption losses; too small of a bandgap results in large thermalization losses. An optimum exists at a bandgap of $1.4 \mathrm{eV}$ with theoretical efficiency of about $33 \%$. Silicon (Si), a commonly used light absorber, on the other hand, has a bandgap of only $1.1 \mathrm{eV}$ and therefore its theoretical efficiency is capped at $29 \%$ for a simple single junction device. Other absorbers exist that are better matched to the solar spectrum, but they are more costly due to the smaller scale of production and availability of materials (see Figure 2.2) [5].

Additionally, silicon still has relatively low absorption, mainly due to it being an indirect bandgap semiconductor (Figure 2.3(c)) [6]. In a direct bandgap semiconductor, such as gallium arsenide (GaAs), the maximum energy of the valence band and the minimum of the conduction band occur at the same wavevector (Figure 2.3(a)), requiring only a photon of an appropriate energy for the electron transition from the valence to the conduction band. For an indirect bandgap semiconductor, such as silicon, the band extrema are offset in wavevector (Figure 2.3(b)), requiring both a photon and a phonon for the transition to occur. As a result, the recombination in $\mathrm{Si}$ is considerably lower than in GaAs, however, the absorption coefficient is also much smaller. Therefore, thicker Si substrates are required to absorb the same amount of solar radiation as compared to those of GaAs. This problem can be effectively mitigated by trapping light in the Si substrate for longer by strategically nanostructuring the surface.

A bare polished $\mathrm{Si}$ wafer has a reflectivity of $>30 \%$ across the visible solar spectrum. Traditionally, anti-reflective coating stacks have been used to minimize this 


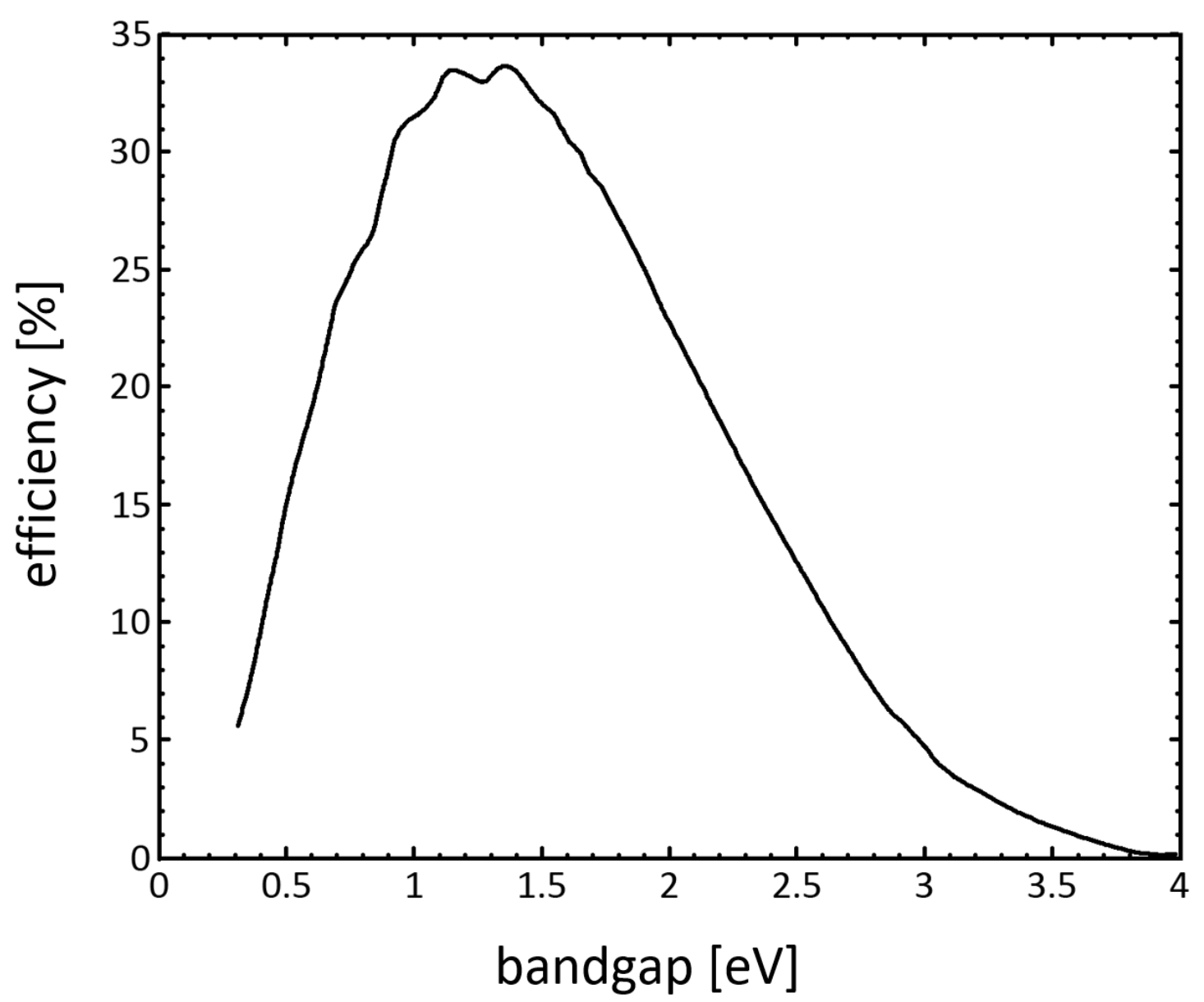

Figure 2.2: Dependence of the maximum single junction solar cell efficiency on the material bandgap. The actual device efficiency is likely to be lower due to various optical and electrical losses. Efficiencies for irradiation at the Earth's surface (AM1.5) are shown. After [7]. 


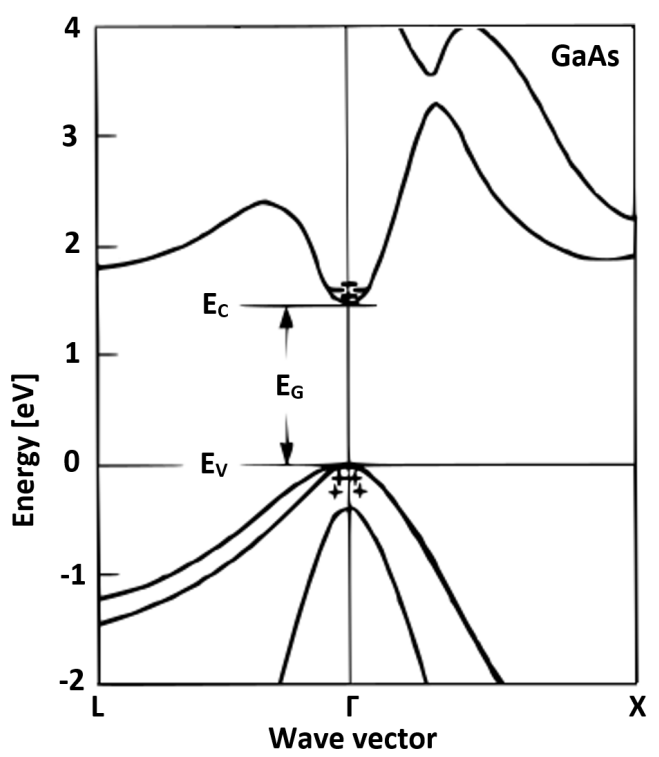

(a) Direct bandgap

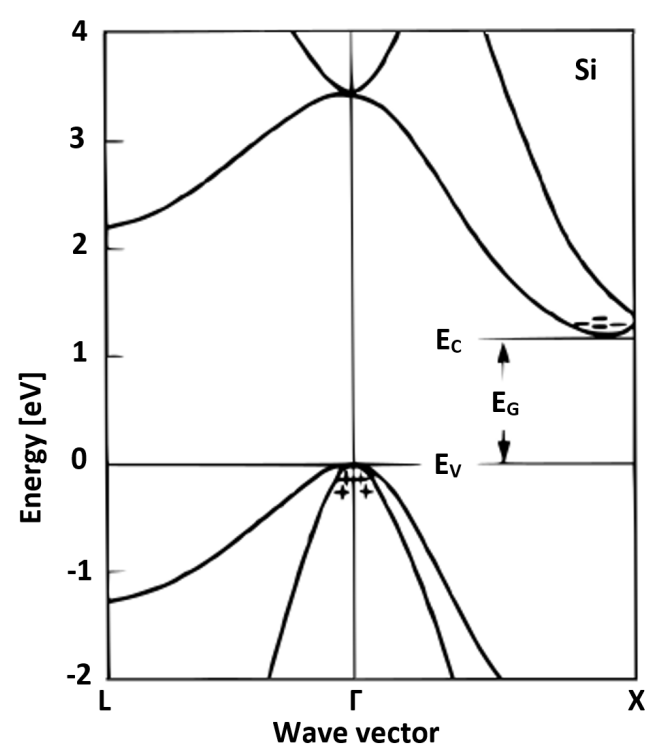

(b) Indirect bandgap

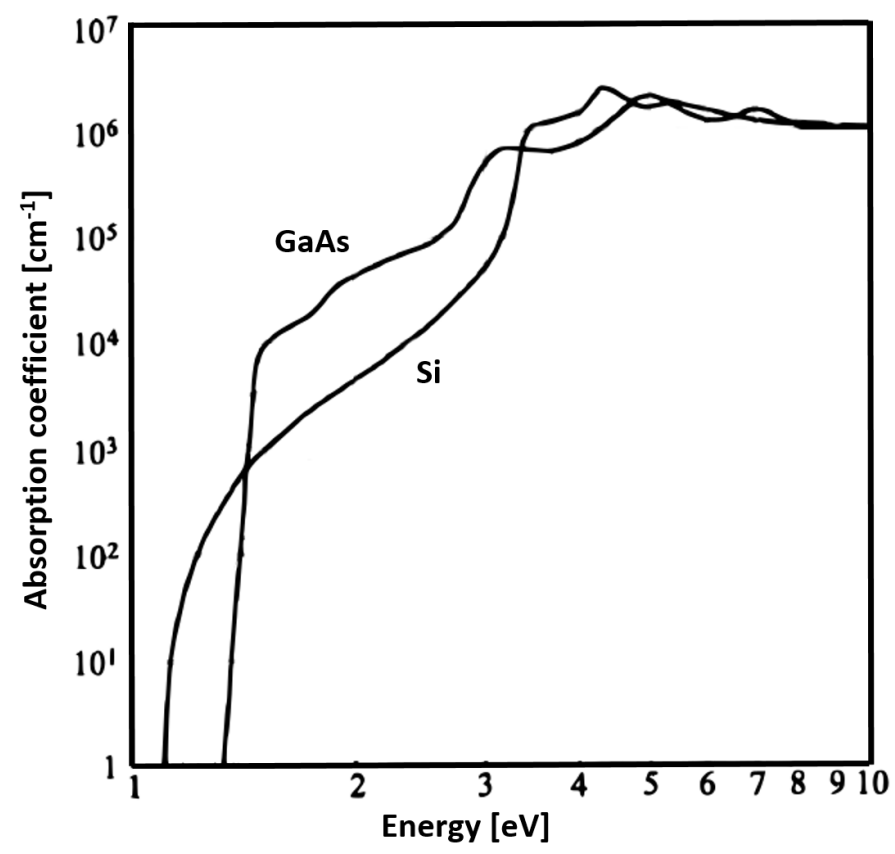

(c) Absorption coefficients for a direct (GaAs) and indirect (Si) semiconductor at $300 \mathrm{~K}$

Figure 2.3: Bandstructures and absorption coefficients for direct and indirect semiconductors. After [8]. 
loss [9]. Anti-reflection coatings are stacks of thin dielectric layers that are deposited onto the solar cell in post processing to better match the refractive index of the ambient to that of the solar cell material. These coatings add both extra processing and material costs in solar cell manufacturing. Nanostructuring the device surface can effectively mitigate this problem as well and will be discussed further in Chapter 4 .

Recombination in $\mathrm{Si}$ is generally facilitated by trap levels in the bandgap caused by impurities or irregularities in the crystal lattice. In the bulk, such defects can be minimized by using Float-zone or Czochralski Si wafers. Both of these Si growth techniques are time and energy intensive, but they produce high-quality crystals. At the surfaces of the wafers, however, irregularities in the lattice, such as dangling bonds are inevitable. An effective counter-measure for surface recombination is tying up these bonds with either elemental hydrogen or a passivating dielectric layer. A thin passivating layer has several other beneficial effects as will be discussed in Section 2.2 .

The solar cell efficiency can be quantified using the current-voltage (IV) characteristics of the cell. In the dark, the IV characteristic of a solar cell is that of a diode. Under illumination, the IV curve is translated down the current axis in proportion to the illumination with the value at the applied voltage of zero volts, termed short circuit current, $I_{s c}$ as represented by equation 2.2 and shown in Figure 2.4. $I_{o}$ is the dark reverse saturation current of the diode; $V$ is the applied voltage; $q$ is the magnitude of the electronic charge; $k$ is the Boltzmann constant; $T$ is the temperature of the system; $n$ is termed the ideality factor and reflects the relative importance of generation/recombination in the depletion region (and other current transport mechanisms) of the diode to drift-diffusion.

$$
I=I_{o}\left(e^{\frac{q V}{n k T}}-1\right)-I_{s c}
$$




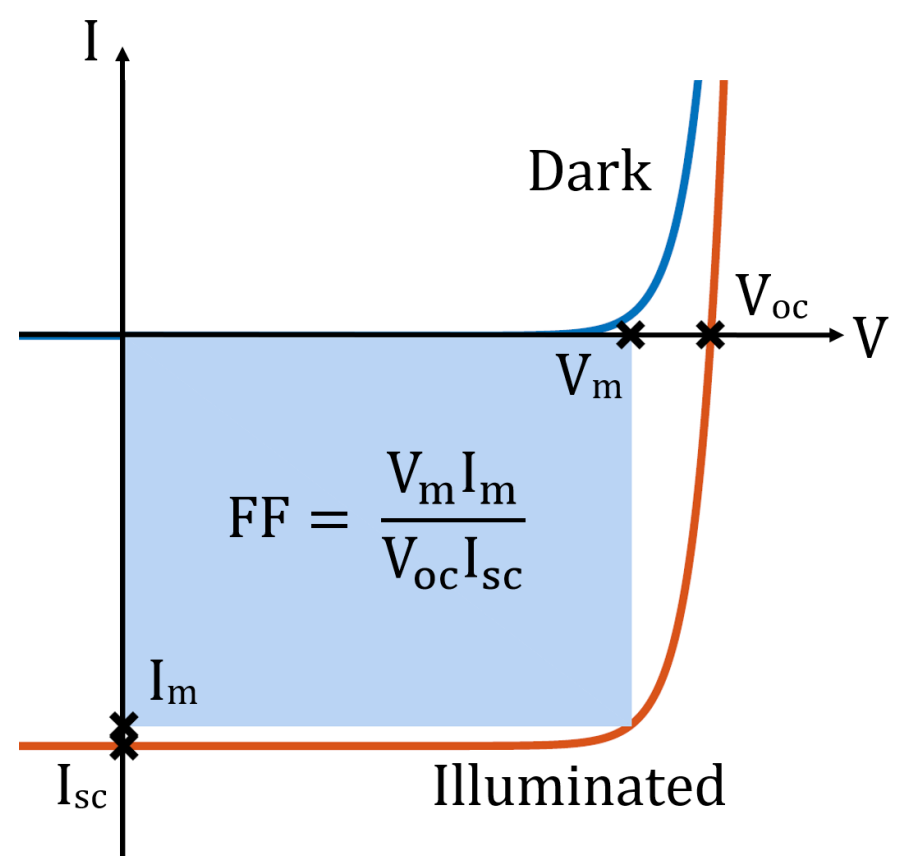

Figure 2.4: Dark and illuminated IV characteristics for a solar cell with $I_{s c}, V_{o c}$ and $F F$ indicated.

The intercept of this curve with the voltage axis gives the open circuit voltage, $V_{o c}$. It should be noted that the open circuit voltage is proportional to the logarithm of the inverse of the saturation current of the photovoltaic diode, $I_{o}$, as shown in equation (2.3). It becomes important to minimize the reverse saturation current to maximize the solar cell efficiency. Even though equation (2.3) implies that the $V_{o c}$ increases with photocurrent, $V_{o c}$ is fundamentally limited by the bandgap of the absorber.

$$
V_{o c}=\frac{n k T}{q} \ln \left(\frac{I_{s c}}{I_{o}}+1\right)
$$

Another figure of merit often quoted in the literature is the fill factor, $F F$, as defined in Figure 2.4. It is susceptible to the shunt and series resistance losses in the cell. The efficiency of the solar cell can be expressed through these parameters as:

$$
\eta=\frac{V_{o c} I_{s c} F F}{P_{\text {optical }}}
$$


In designing a solar cell, the $V_{o c}, I_{s c}$ and $F F$ should be optimized for the maximum device efficiency.

\subsubsection{Grid Parity}

Although the efficiency of the photovoltaic devices is important, the practical quantity of interest is the cost per unit energy produced. Nationwide in Canada, the electricity rates vary between approximately $7 \mathrm{c} / \mathrm{kWh}$ and $18 \mathrm{c} / \mathrm{kWh}[10,11]$. Elsewhere in the world, the electricity prices range from $3 \mathrm{c} / \mathrm{kWh}$ to over $40 \mathrm{c} / \mathrm{kWh}$ [4]. Thus to be viable for terrestrial applications, a photovoltaic technology must approach and beat these prices. For a crystalline-Si solar cell with efficiency of about $10 \%$, the cost of the system is approximately $\$ 3 / \mathrm{W}$, which over 20 years gives an average electricity cost of 7-17 $\mathrm{c} / \mathrm{kWh}$ depending on amount of sunshine received a day. This would make the technology attractive in countries like Denmark and Germany with grid electricity costs over $30 \mathrm{c} / \mathrm{kWh}[4]$. Some improvements in system costs and/or efficiencies are needed to entice North America to adopt photovoltaics. As a result, the photovoltaic research has diverged, some seeking to maximize efficiency, others to minimize cost.

\subsubsection{State of the Industry}

The 2016 overview of the photovoltaic industry by the National Renewable Energy Laboratory (NREL) is shown in Figure 2.5. Currently, the highest efficiency solar cells, aside from using exotic materials, use multi-junction technology. Such a device consists of several cells stacked on top of each other with progressively smaller bandgaps moving from the front to the back of the device. This architecture allows a broader range of the solar spectrum to be used with less thermalization loss and can theoretically be used with any set of material with appropriate bandgaps. However, the additional processing and fabrication equipment cost drives up the price of 
such devices considerably [4]. Of single junction solar cells, it is hard to compete with the efficiency of the direct-bandgap semiconductors like GaAs with a maximum

reported efficiency of $28.8 \%$ (Alta Devices) [12]. Such materials remain relatively expensive and not commercially feasible for terrestrial applications, except maybe in concentrator-based systems. Single-crystal silicon is a close second with $25 \%$ efficiency [12]. As a widely produced material for the integrated circuit industry it is considerably more cost effective. Until recently, silicon-based solar cells could not compete with the price of the energy from the grid in Canada. As a more cost effective alternative, solar cells based on multicrystaline, microcrystalline, and thin-film silicon have been under development [12]. For the past decade, the Cadminum Indium Galium Selenide (CIGS) and cadmium telluride (CdTe) cells have been hailed as the front running candidates to replace Si cells with efficiencies reaching nearly $20 \%$ [12] and commercially friendly roll-to-roll manufacturing process. However, some of the materials required in the manufacturing process are quite toxic and others are in limited supply [13]. Dye sensitized cells, one of the earliest conceived hybrid organic/inorganic photovoltaic technologies has made relatively slow progress since its inception in 1988, with reported efficiencies of nearly $12 \%$. The relatively new area of organic solar cells has been growing rapidly with efficiencies increasing from $3 \%$ to over $10 \%$ in merely a decade $[12,14,15]$. The newcomer on the scene is a Perovskite solar cell with efficiencies of over $22 \%$ [12].

\subsection{MIS Devices}

The device under investigation in this work uses a metal-insulator-semiconductor (MIS) architecture. Such a device is based on a Schottky junction - a rectifying junction created when a metal and a semiconductor are brought into contact. The difference in the Fermi levels in the metal and semiconductor is what creates the 


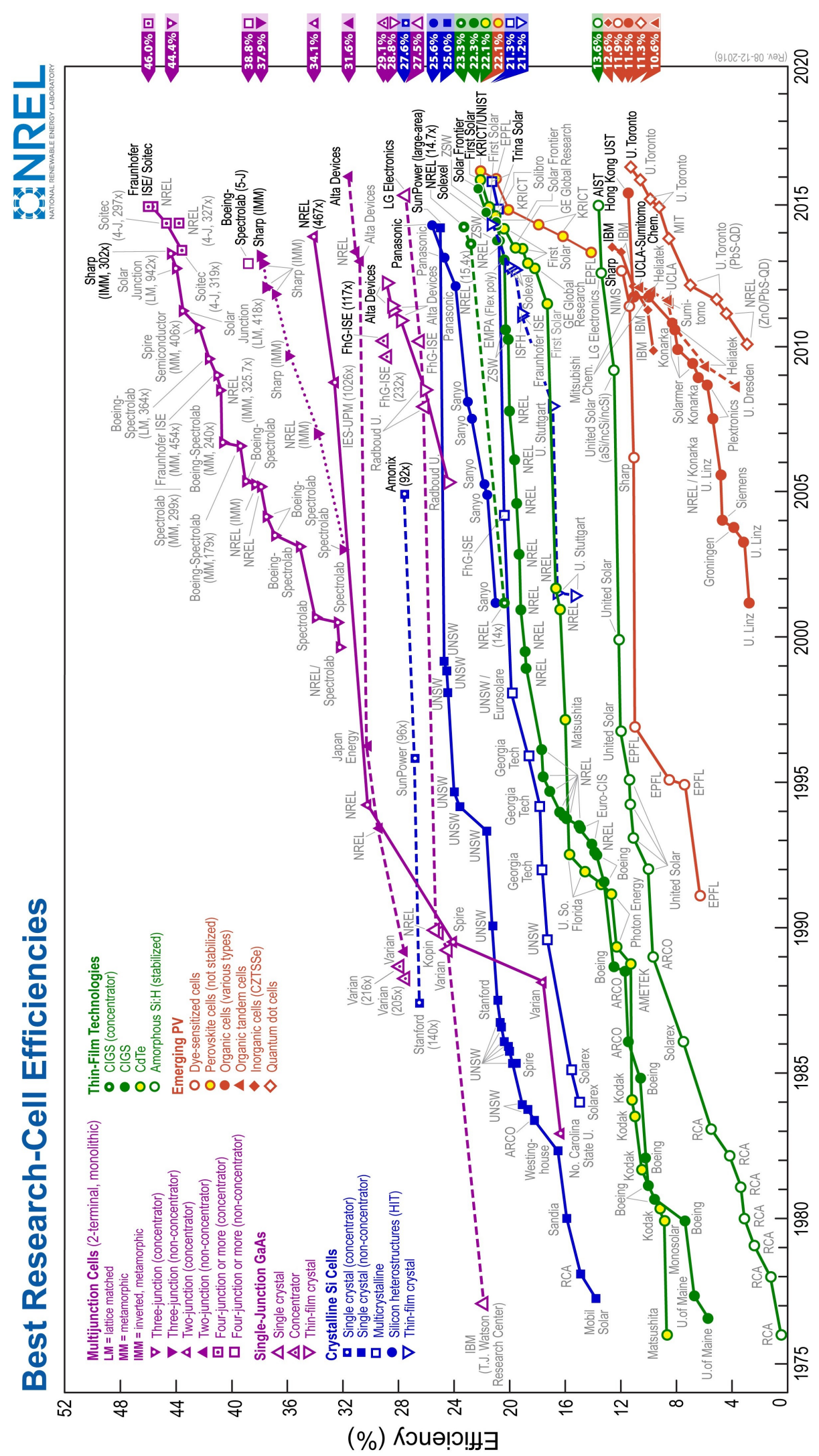

Figure 2.5: The 2016 NREL survey of the state of the photovoltaic industry. 


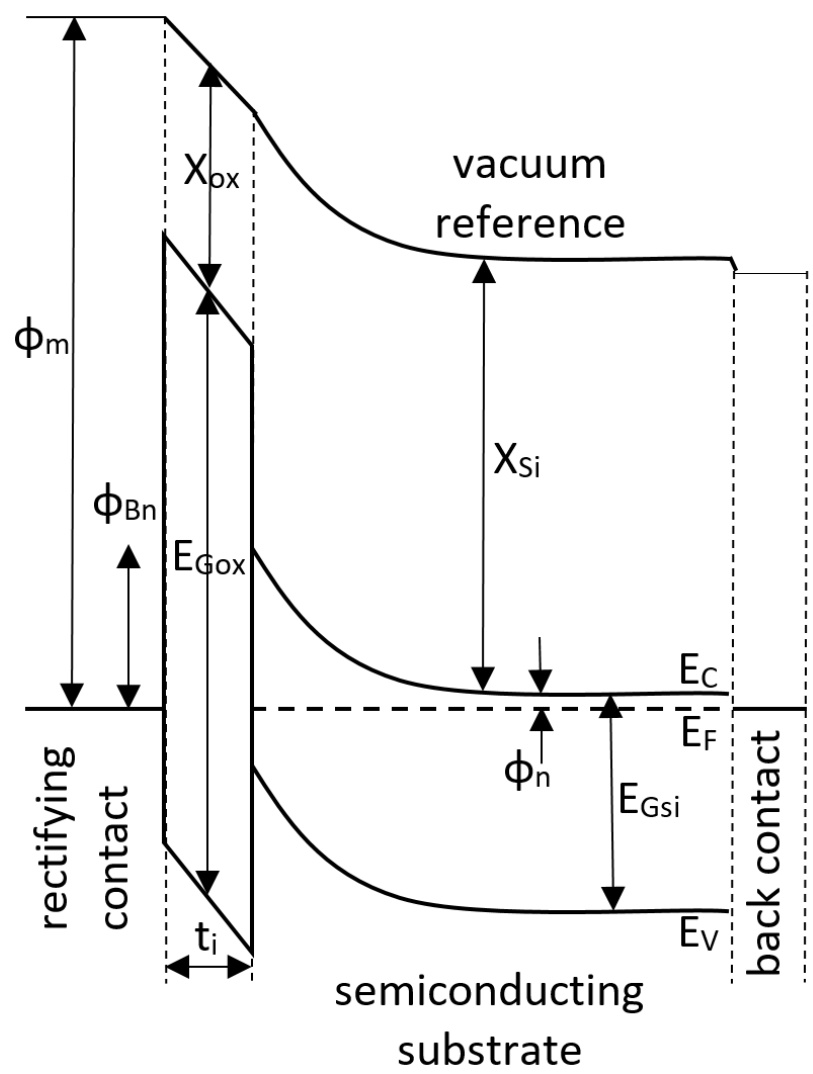

Figure 2.6: Band diagram of an MIS device with various relevant variables labelled.

depletion region. The effect of adding the thin insulator layer is two-fold. It increases the effective built-in voltage of the diode, reducing the dark reverse saturation current. This allows for a higher conversion efficiency. Additionally, the thin dielectric reduces the majority carrier current, allowing the diode to operate as a minority carrier device $[8,16]$. The band diagram of such a device is shown in Figure 2.6.

The operation of these MIS devices is greatly influenced by the work function of the metal, $\phi_{m}$, and the thickness of the insulator, $t_{i}$. Based on the thickness of the insulator the devices can be divided into equilibrium $\left(t_{i}>5 \mathrm{~nm}\right)$ and nonequilibrium $\left(t_{i}<5 \mathrm{~nm}\right)$ devices. The operation of the equilibrium devices is tunnelcurrent limited. This small tunneling current is not enough to disturb the electrostatic potential throughout the diode. Such devices behave like leaky metal-oxidesemiconductor (MOS) capacitors and are not amenable to photovoltaic applications. 
In non-equilibrium devices with sufficiently thin insulators the current becomes driftdiffusion limited [17]. These devices approach the operation of a $p n$ junction, and as such have great potential for photovoltaics [18].

Based on the metal work function, the non-equilibrium devices can be divided into majority and minority carrier diodes. The majority carrier flow will always remain tunnel-limited, since the majority carriers have no difficulty passing through the semiconductor. The minority carrier flow, on the other hand, can be semiconductorlimited. To create a minority carrier MIS device, the work function of the metal needs to be chosen so that the surface of the semiconductor is inverted at thermal equilibrium. At the same time, the insulator needs to be thin enough to prevent tunnel-limiting the minority carrier current over a range of biases. With a proper selection of the metal and insulator thickness an MIS diode with current and capacitance characteristics approaching those of a pn junction can be generated [18].

The condition for weak inversion in a semiconductor is established when the surface potential, $\phi_{S}$, exceeds the potential difference between the intrinsic and doped Fermi levels in the semiconductor in the bulk, $\phi_{B}$ defined by equation 2.5, as shown in Figure 2.7). The onset of strong inversion happens when the surface potential exceeds $2 \phi_{B}$.

$$
\phi_{B}=\frac{k_{B} T}{q} \ln \left(\frac{N_{D}}{n_{i}}\right)
$$

where $k_{B}$ is the Boltzmann constant, $T$ is the temperature of the semiconductor, $q$ is the electronic change, $N_{D}$ is the doping density of the semiconductor, and $n_{i}$ is the intrinsic carrier density [8].

For $n$-type Si with doping of $1 \times 10^{15} \mathrm{~cm}^{-3}$, weak inversion can be established using a metal with a work function $\phi_{m}>4.59 \mathrm{eV}$ and strong inversion can be achieved with a metal work function of $\phi_{m}>4.88 \mathrm{eV}$ (as a coarse estimate, disregarding the potential 


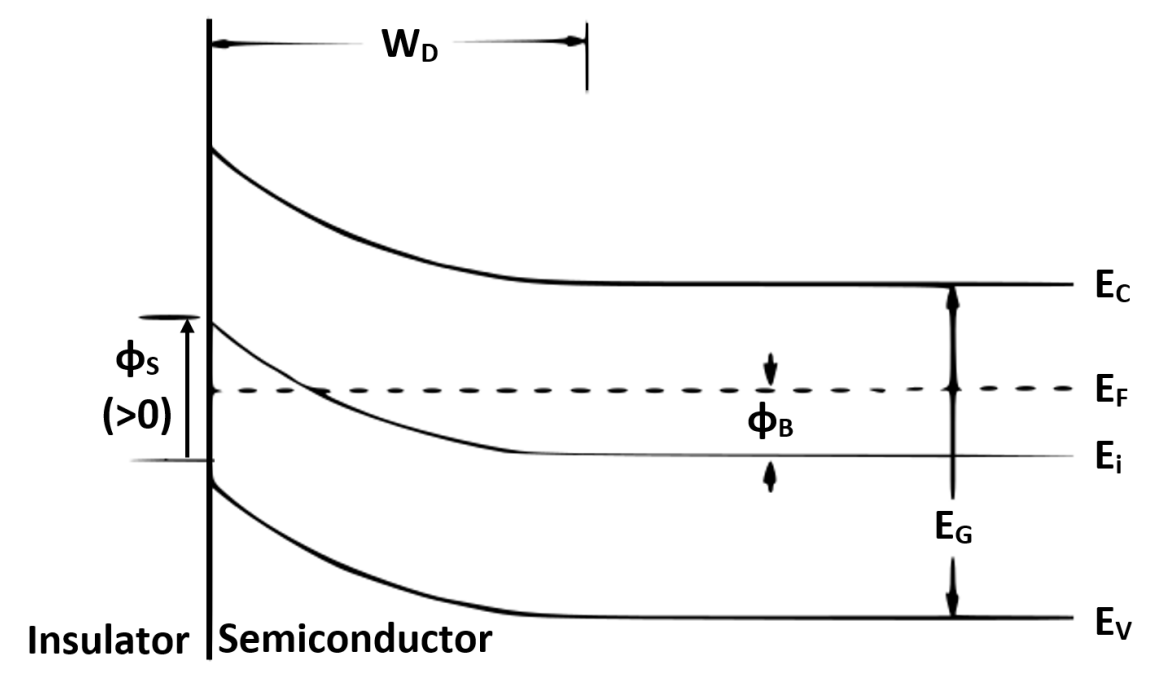

Figure 2.7: Energy-band diagram at the surface of an $n$-type semiconductor. The reference for the potential energy is taken to be the intrinsic Fermi level in the bulk of Si. Weak inversion occurs when the surface potential, $\phi_{S}$, exceeds $\phi_{B}$ as defined in this figure. Strong inversion is said to occur for $\phi_{S}>2 \phi_{B}$. After [8].

drop across the interfacial oxide).

Interface states can also influence the operation of an MIS diode by providing additional paths for current flow between the metal and the semiconductor as shown in Figure 2.8. The presence of surface states results in a higher collected current, which is especially evident in the depletion region of operation of an MIS device, where the tunneling current components are reduced $[18,19]$.

The MIS structures for photovoltaics were a popular research topic in the 1970s and 1980s due to their economical advantage over the conventional $p n$ junction solar cells. However, these structures suffered from poor efficiencies due to the reflection and poor transmission through the top metal contact. The research was all but abandoned until the transparent conductive oxides (TCO) presented a viable alternative. Indium-tin-oxide (ITO) is an obvious choice for a transparent conductive layer for MIS formation. Such devices have indeed been fabricated on crystalline and porous silicon substrates with efficiencies of approximately $10 \%[20,21]$. Aside from evaporation, 


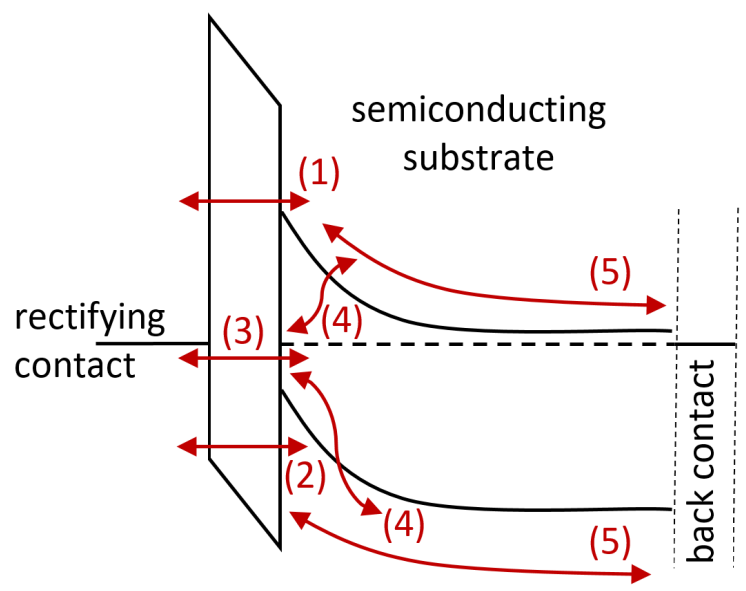

Figure 2.8: Band diagram of an MIS device showing possible current paths (1) metal - conduction band tunneling (2) metal - valence band tunneling (3) metal - surface states tunneling (4) surface states - conduction/valence band recombination (5) drift - diffusion.

sputtering, and chemical vapour deposition (CVD) a variety of low energy, cost effective deposition processes are available for ITO, such as spray pyrolysis, sol-gel spin casting, and nanoparticle spin casting [20-23]. However, the material itself remains expensive with an unreliable supply chain due to the limited supply of indium [14].

Recently, other transparent conductive oxides have been investigated. Some of them do not contain toxic or rare materials [24]. One such material is tin oxide, $\mathrm{SnO}_{2}$. MIS devices of $\mathrm{SnO}_{2} / \mathrm{Si}$ have been reported in the past with efficiencies of $12.5 \%$ and a maximum theoretical efficiency of $20 \%$ [25]. These devices would be direct competitors to hybrid solar cells which use a transparent organic conductor instead. However, there are also processing and production considerations to take into account. Traditionally, transparent conductive oxides have been sputter-deposited in vacuum in batches. The development of roll-to-roll planar magnetron sputtering made the conductive oxides commercially viable, however, vacuumless deposition would be more economical $[26,27]$. Vacuumless deposition of these materials has been explored for years with such techniques as spray pyrolysis and sol-gel technology, however, the conductivity tends to suffer $[20,26,28]$. 


\subsection{Si Nanostructuring}

$\mathrm{Si}$ is quite efficient at absorbing the blue wavelengths of the solar spectrum, however, most of the red spectrum passes through a relatively thin Si device unabsorbed. To make better use of this available energy, the surface of Si can be roughened to create multiple reflections back into the substrate.

The most common and a rather elegant way of nanostucturing a Si surface is an anisotropic wet alkaline etch. A variety of etchants are possible including $\mathrm{NaOH}$, $\mathrm{Na}_{2} \mathrm{CO}_{3}, \mathrm{KOH}$ and tetramethylammonium hydroxide (TMAH) [29]. It is an economical and commercially viable technique of creating a pyramidal structure on the $\mathrm{Si}$ surface. These structures are on the order of microns in size and are generally analysed using geometrical ray tracing techniques. Various sources report the minimum reflection of such a structured surface at approximately $10 \%$ in the optical range. As such, an anti-reflection coating is still used with such pyramidal structures to minimize the reflection even further [6,29-31]. However, high aspect ratio structures with feature sizes on the order of the optical wavelengths can create optically black surfaces without the need for anti-reflection coatings.

When the feature size of the surface structuring is smaller than the wavelength of the incident optical wave, the light only sees the effective permittivity of the surface and not the geometry of the structure. In this case, if the effective permittivity can be varied continuously from that of the ambient to that of the substrate, the reflection can be greatly minimized. This effect can be achieved by continuously increasing the volume fraction of the substrate material from 0 to $100 \%$ throughout the surface structure, effectively producing a small-scale pyramidal structure as illustrated in Figure 2.9.

The transmission and reflection properties of this nanostructured surface can be 


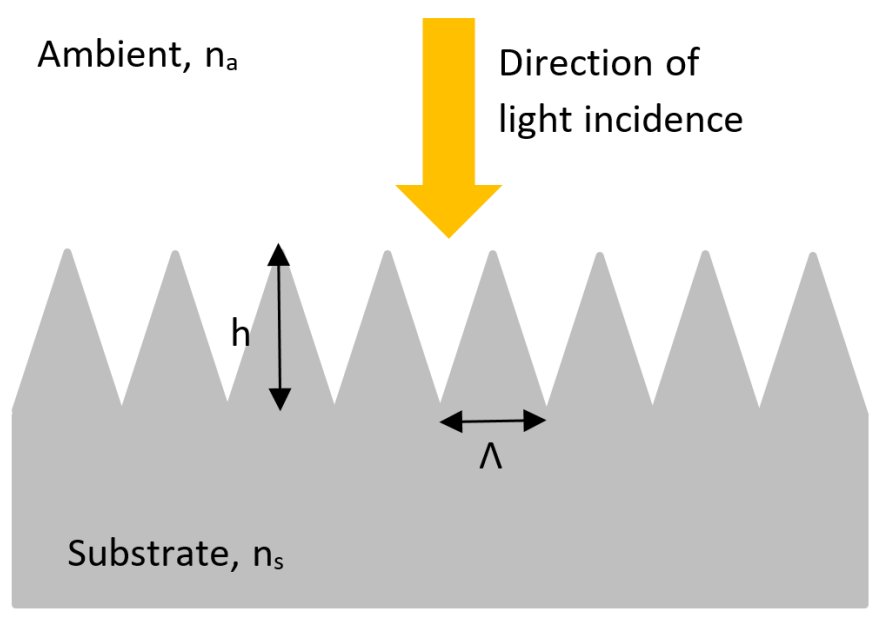

Figure 2.9: Sketch of a nanostructured surface with relevant variables labelled.

well described by the effective medium theory. There are several approaches to estimating the effective medium permittivity.

$$
\begin{array}{lr}
\frac{\epsilon_{e f f}-\epsilon_{2}}{\epsilon_{e f f}+2 \epsilon_{2}}=f(x) \frac{\epsilon_{1}-\epsilon_{2}}{\epsilon_{e f f}+2 \epsilon_{2}} & \text { Maxwell-Garnett } \\
f(x) \frac{\epsilon_{1}-\epsilon_{e f f}}{\epsilon_{1}+2 \epsilon_{e f f}}+(1-f(x)) \frac{\epsilon_{2}-\epsilon_{e f f}}{\epsilon_{2}+2 \epsilon_{\text {eff }}}=0 & \text { Mean-field self-consistent } \\
\epsilon_{\text {eff }}^{(0)}=f(x) \epsilon_{2}+(1-f(x)) \epsilon_{1} & \text { Rytov, } 0^{\text {th } \text { order }} \\
\epsilon_{\text {eff }}^{(2)}=\epsilon_{\text {eff }}^{(0)}\left[1+\frac{\pi^{2}}{3}\left(\frac{\Lambda}{\lambda}\right)^{2} f^{2}(1-f)^{2} \frac{\left(\epsilon_{2}-\epsilon_{1}\right)^{2}}{\epsilon_{e f f}^{(0)}}\right] & \text { Rytov, } 2^{\text {nd }} \text { order }
\end{array}
$$

where $\epsilon_{1}$ and $\epsilon_{2}$ are the permittivities of the inter-penetrating materials, in this case air and silicon respectively; $\epsilon_{e f f}$ is the effective permittivity of the structure; $f$ is the volume fraction of the second material; $\Lambda$ is the periodicity of the structure as defined in Figure 2.9; $\lambda$ is the wavelength of the incident light.

Equations Maxwell-Garnett (2.6) and Rytov, $0^{\text {th }}$ order (2.8) have limited applicability with the Maxwell-Garnett equation being applicable only for low volume 
fractions of the substrate material [32] and Rytovs $0^{\text {th }}$ order equation being applicable only when the structure periodicity is much smaller than the wavelength of interest $(\Lambda<<\lambda)[33-35]$.

For minimizing the reflection of the nanostructured surface over the optical range of interest (around 300-1100 nm), the height of the nanostructure needs to be on the order of $1 \mu \mathrm{m}$. The feature size of this structuring should be on the order of the wavelength or below for the effective medium theory to apply. Ideally the volume fraction of Si would also increase continuously toward the substrate to minimize reflection. An ideal surface structure can be modelled as a forest of cones. This allows the effective surface area to be increased by as many as 5-10 times.

However, the surface inherently contains many more recombination centers than the bulk of the material due to the abrupt end of the crystal lattice and thus many unsatisfied bonds, termed "dangling" bonds. These dangling bonds along with any impurities introduced during processing are responsible for a continuum of interface or surface states that exist in the bandgap. These surface states act as recombination centers reducing the amount of generated photocurrent in a solar cell, and reducing cell efficiency. The surface recombination rate is defined analogously to the ShockleyHall-Read recombination [36]:

$$
U_{s}=\int_{E_{V}}^{E_{C}} \frac{\sigma v_{t h}\left(p_{s} n_{s}-n_{i}^{2}\right) D_{i t}}{n_{s}+n_{i} e^{\left(E_{T}-E_{i}\right) / k T}+p_{s}+n_{i} e^{-\left(E_{T}-E_{i}\right) / k T}} d E
$$

Here $p_{s}$ and $n_{s}$ are the surface hole and electron concentrations respectively; $n_{i}$ is the intrinsic carrier density; $D_{i t}$ is the energy dependent surface state density; $v_{t h}$ is the thermal velocity; and $\sigma$ is the capture cross section. Under one sun illumination and a moderate bias (the expected operation conditions of a solar cell), it can be reasonably expected that only the surface states near the mid-gap will be effective recombination centers [8]. As such, the surface state density can be approximated as 
a constant with the value at mid-bandgap.

$$
U_{s}=\frac{\sigma v_{t h}\left(p_{s} n_{s}-n_{i}^{2}\right) \overline{D_{i t}} E_{G}}{n_{s}+p_{s}+2 n_{i}}
$$

Assuming that the carrier density at the surface is dominated by the photogenerated charge, the recombination rate can be further simplified ${ }^{2}$. A concept of a surface recombination velocity, $S$, is introduced, defined in the equation below:

$$
U_{s}=\frac{S p_{g}}{2}=\frac{\sigma v_{t h} \overline{D_{i t}} E_{G} p_{g}}{2}
$$

Here $p_{g}$, is the carrier density generated at the surface by illumination. The recombination current density, $J_{r}$, then becomes:

$$
J_{r}=q U_{s}
$$

Being directly proportional to the interface state density, recombination current can increase by up to an order of magnitude due to the anti-reflective surface nanostructuring. As such, surface passivation becomes very important. It is possible to minimize loss due to recombination by accumulating charge at the surface (or inverting the surface), thereby reducing the efficiency of the recombination centers at the interface. For $n$-type substrates, this inversion can be enforced by intentionally introducing fixed negative charge into the insulator at the Si surface [37]. The model in equation (2.12) cannot account for this effect. To better model the real behavior of the surface states, a concept of an effective surface recombination velocity is introduced, $S_{\text {eff }}$, defined at the edge of the depletion region, instead of the surface. In this case, the recombination rate depends on this effective surface recombination velocity and the excess charge carriers at the edge of the depletion region:

\footnotetext{
${ }^{2}$ Given the assumptions made, as well as the large range of $D_{i t}$ and $\sigma$ reported in the literature only an order-of-magnitude approximation to the surface recombination rate can be obtained.
} 


$$
U_{s}=S_{e f f} p_{g}(x=W)
$$

A single junction silicon solar cell is theoretically capable of generating a maximum of $33 \mathrm{~mA} / \mathrm{cm}^{2}$. Based on the simulation of an MIS solar cell without interface states under one sun illumination the hole density at the depletion region edge is expected to be below $10^{14} \mathrm{~cm}^{-3}$. The effective surface recombination velocity of an unpassivated $n$-type Si wafer can range between $1000 \mathrm{~cm} / \mathrm{s}$ to $10000 \mathrm{~cm} / \mathrm{s}$ [38], giving a recombination current of $J_{r}$ around $16 \mathrm{~mA} / \mathrm{cm}^{2}-160 \mathrm{~mA} / \mathrm{cm}^{2}$, completely obliterating any short circuit current, and reducing the cell efficiency to 0. However, with an optimized thermally-grown $\mathrm{SiO}_{2}$ passivation with intentional inclusion of fixed negative charge in the oxide $S_{\text {eff }}$ below $1 \mathrm{~cm} / \mathrm{s}$ should be possible [39]. Even $\mathrm{Al}_{2} \mathrm{O}_{3}$ deposited by atomic layer deposition (ALD, discussed later in this chapter) has been shown to result in excellent passivation with $S_{\text {eff }}$ approaching $10 \mathrm{~cm} / \mathrm{s}$ even for film thicknesses of a few nanometers. The passivation tends to be better on $n$-type $\mathrm{Si}$ possibly due in part to the inherent inclusion of negative charge in the $\mathrm{Al}_{2} \mathrm{O}_{3}$ film during the ALD process [40-42]. These passivation levels would lead to recombination currents densities of around $0.016 \mathrm{~mA} / \mathrm{cm}^{2}$ to $0.16 \mathrm{~mA} / \mathrm{cm}^{2}$. Even if increased tenfold due to the surface structuring, a significant portion of the photogenerated charge should still be collected.

\subsection{Insulator Layer}

As mentioned previously, the thickness of the insulator layer is another crucial design parameter of the MIS diode. The insulator needs to be thick enough to increase the effective potential barrier, reducing the reverse saturation current and increasing the open circuit voltage of the solar cell thereby increasing its efficiency. On the other hand, the insulator needs to be thin enough to allow sufficient tunneling current flow. 


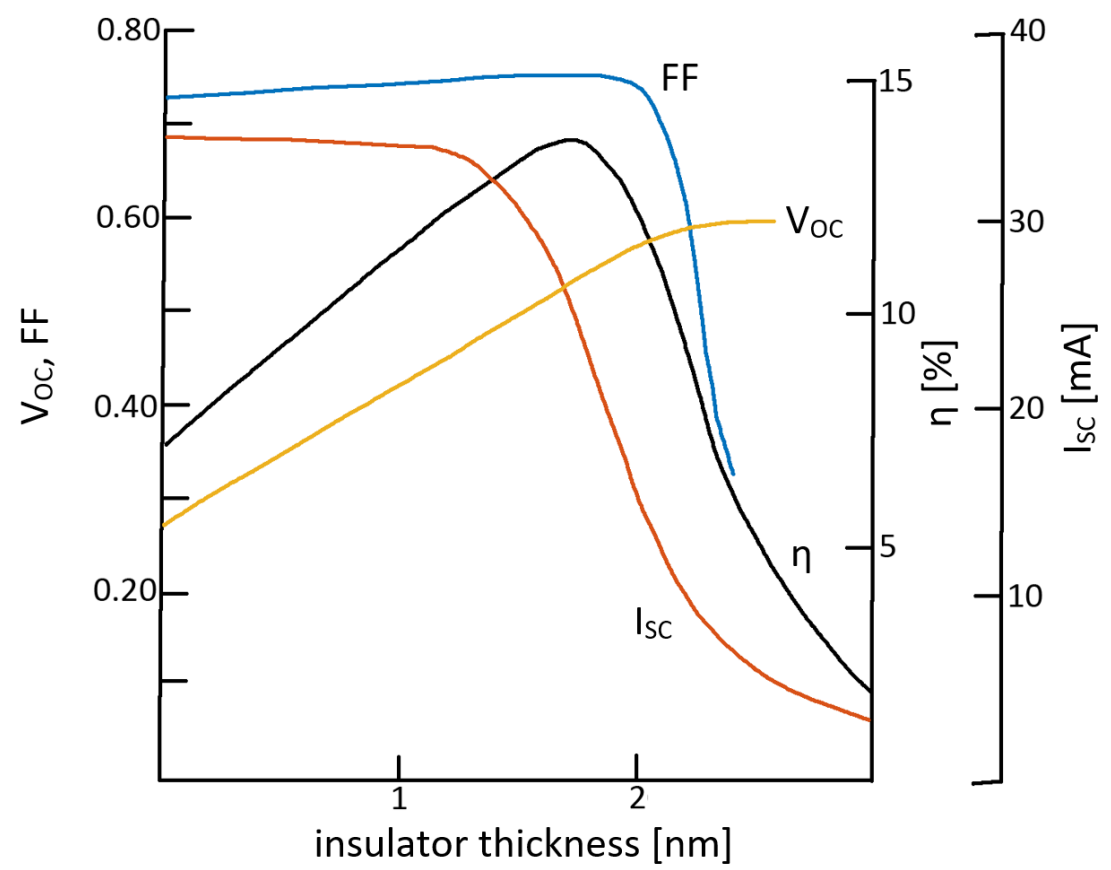

Figure 2.10: Effect of insulator layer thickness on the parameters of a photovoltaic cell. After [43].

It has been found that the required dielectric thickness for $\mathrm{SiO}_{2}$ on a $\mathrm{Si}$ substrate is on the order of 1-2 nm as shown in Figure 2.10 [43].

In early MIS device research, native oxide was used as the tunneling insulator layer because its growth naturally saturates in the required range of a few nanometers [44-46]. However, such a hands-off $\mathrm{SiO}_{2}$ growth method provides little control of the $\mathrm{Si} / \mathrm{SiO}_{2}$ interface and tends to result in high interface state densities [47], which will cause recombination at the surface and reduce the efficiency of the solar cell.

In contrast, thermally-grown dielectrics are known to have excellent passivation properties resulting in low interface state densities $[48,49]$. However, the various existing oxidation models do not have very good predictive power at oxide thicknesses of a few nanometers. Additionally, the initial surface properties (roughness, terminating species, etc.) have a large effect on the oxidation dynamics when the oxide is thin, making it difficult to predict the oxidation rates [49-56]. These limitations may not 
exclude the use of thermally-grown oxide altogether. However, they may warrant a consideration of deposited dielectrics. Additionally, deposited dielectrics afford the use of a much larger variety of insulator materials, possibly allowing for thicker oxides and better fabrication tolerances without compromising carrier tunneling.

For successful MIS devices, the dielectric layer must be continuous and conformal. A growth technique called atomic layer deposition (ALD) might be the key to making structured MIS devices possible, as it provides the needed conformality and continuity of the film with excellent thickness control on the molecular scale even over textured surfaces. It is a self-limiting chemical growth process, which is performed in cycles of four steps as illustrated in Figure 2.11. First, a vapour is introduced over the surface. It is chemically designed such that it will react with the surface but not with itself, so that only a monolayer is deposited on the surface. Excess vapour is removed. Then a second vapour is introduced. It will react only with the monolayer and not with itself producing a layer of target material one molecule thick. It will also reactivate the surface for the first reaction. This cycle can be repeated as many times as necessary to achieve the desired thickness. As an added benefit, ALD is far less energy intensive then thermal growth, requiring about $300^{\circ} \mathrm{C}$ for 5 - 10 minutes to grow 1-2 nm as compared to thermal growth, requiring $500^{\circ} \mathrm{C}-900^{\circ} \mathrm{C}$ for the same duration [57-59]. With plasma-assisted ALD, the temperatures can be brought down even further [60].

\subsection{Organic Conductors}

The current study is particularly focused on the properties of polymeric organic hole conductors. Even though organic small molecule materials capable of conducting electrons or holes exist, only polymeric organic hole conductors will be discussed here. 


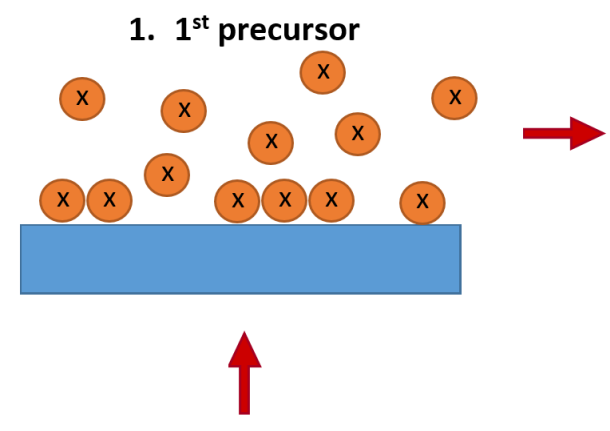

4. Purge

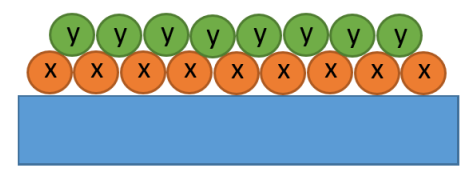

2. Purge

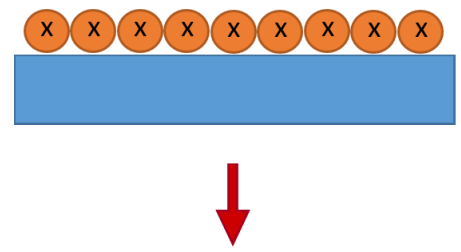

3. $2^{\text {nd }}$ precursor

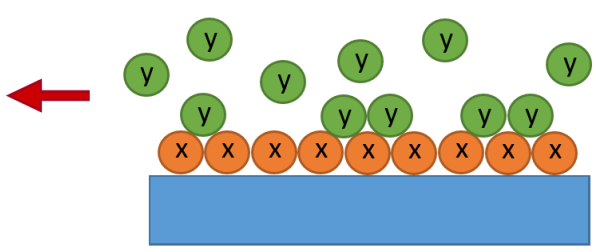

Figure 2.11: Illustration of an ALD process.

The conduction mechanism of an organic conductor is distinctly different from that of inorganic metals and semiconductors. An undoped polymeric chain will form conjugated bonding $\pi$ and anti-bonding $\pi^{*}$ orbitals along its length. These can be modelled as thin valence and conduction bands respectively as shown in Figure 2.12(a).

Organic conductors are expected to have bandwidths on the order of $0.1-0.4 \mathrm{eV}$ depending on the degree of interaction between polymer chains [61]. Since it is not energetically preferable for charge to reside in the anti-bonding orbital, the polymer will conduct only holes. Additionally, unlike in a metal, the carriers (i.e. holes) interact strongly with the polymer chain and deform the chain as they travel along it. This new entity comprised of a hole (or two holes) coupled with a polymer chain distortion is termed a polaron (or a bipolaron). This energy disturbance can be modelled as additional polaron and bipolaron bands that become evident once the polymer is doped [61,62]. Figure 2.13 shows a band diagram sketch of a polymeric conductor. As one might expect, a polaron would move slower along the polymer chain than a hole would travel in a metal due to the interaction with the chain structure. The limiting conduction mechanism does not occur along the polymer 


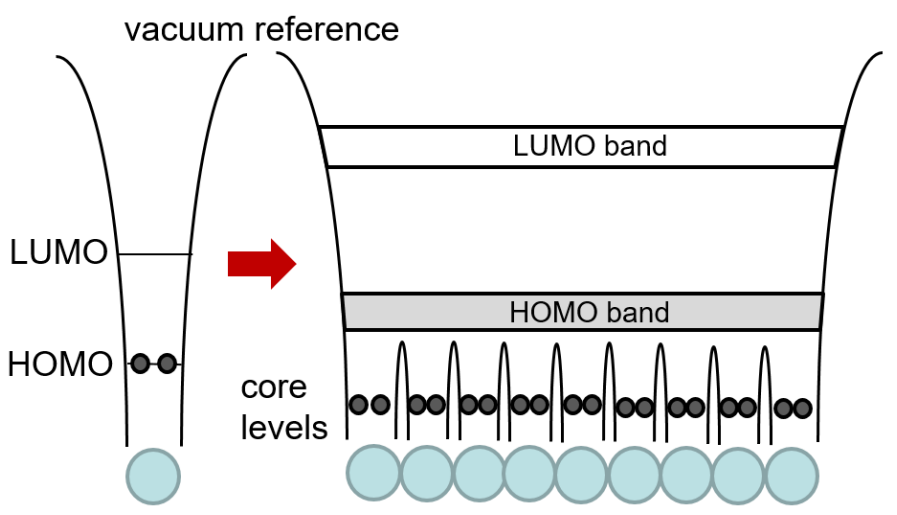

(a)

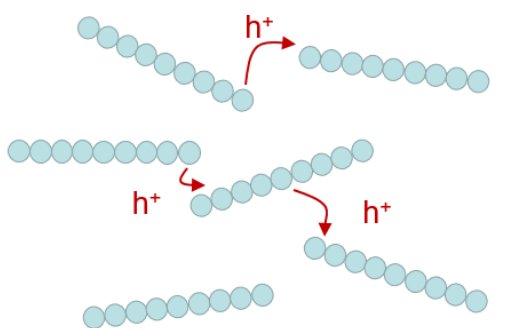

(b)

Figure 2.12: Conduction in polymers. (a) Formation of thin energy bands in polymeric semiconductors. The thickness of the bands depends on the degree of interaction between the molecules in the solid. After [61]. (b) Inter-chain hopping.

chains, but rather between them, where the holes must be transported by a slow, thermally-activated hopping mechanism as shown in Figure 2.12(b).

Despite the above mentioned complexity, polymeric conductors are routinely treated in the literature as conventional metals with overlapping conduction and valence bands $[63,64]$. Not accounting for the finite width of the energy bands inevitably hinders the understanding and predictability of the polymeric device operation.

In this work, the conductive polymer used is poly(3,4-ethylenedioxythiophene), commonly known as PEDOT. It is a stable and well characterized organic conductor used both in the organic electronics and organic photovoltaic industries. It is readily available in a variety of formulations, and can be easily polymerized in situ. However, the most widely used and the easiest to work with formulation is an aqueous dispersion of PEDOT doped with poly(styrenesulfonate) (PSS), termed PEDOT:PSS. Its chemical structure is shown in Figure 2.14. It can be easily deposited onto surfaces by spin-casting [62]. The tradeoff for convenience is in the conductivity. Even though PEDOT can be highly conductive (conductivities up to $1000 \mathrm{~S} / \mathrm{cm}$ have been reported), the PSS backbone is not. It makes the dispersion soluble, but limits the 
LUMO
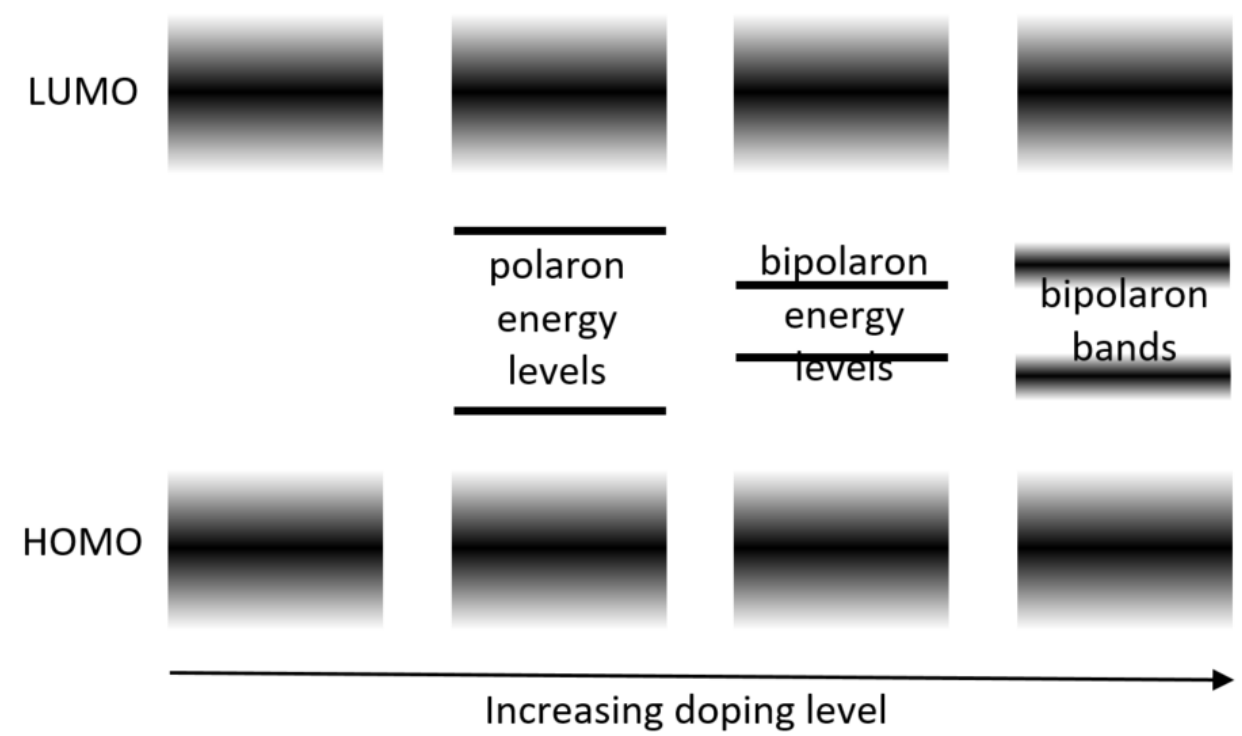

Figure 2.13: Band diagrams of polymeric semiconductors in various states of doping. After [62].

conductivity of the resultant film. However, considerable progress has been made in this area, with formulations available from Heraeus Holdings under the Clevios trademark that are advertised as having conductivities approaching those of in situ films $[65]$.

There have been conflicting reports of the work function of PEDOT:PSS, as can be expected with inconsistent measurement procedures [63]. Recently the scientific

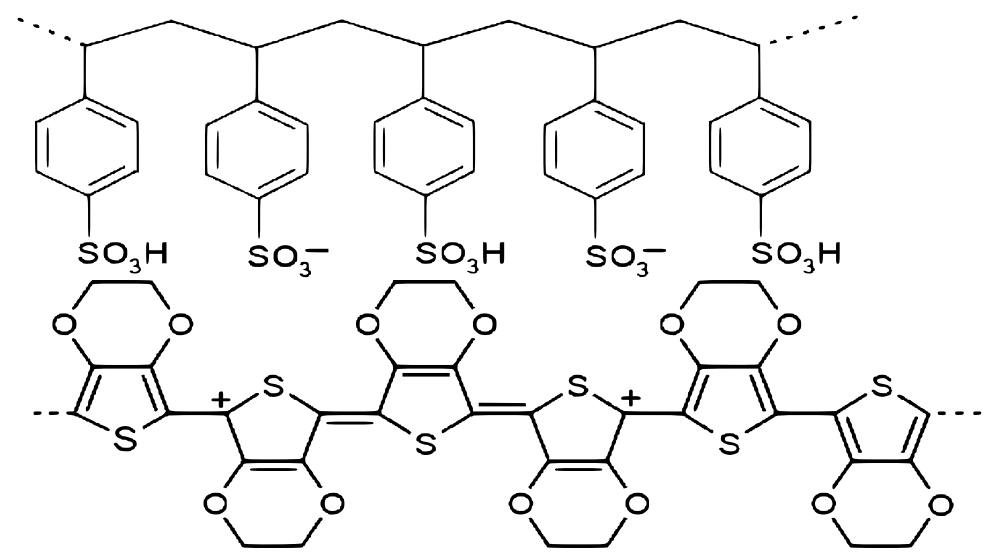

Figure 2.14: Chemical structure of PEDOT:PSS. After [66]. 
community converged on $5.1 \mathrm{eV}-5.2 \mathrm{eV}$ as the work function [62], which would make this material suitable for use in an MIS device. However, the surface preparation and the deposition method of the material can cause considerable differences in the effective work function at the interface (more so than in the case of a conventional metal) due to both the complex structure and the heterogeneity of the material, especially in the case of PEDOT:PSS. At the surface of the PEDOT:PSS film, dipoles are formed between the PEDOT (positively charged) and PSS (negatively charged). If the orientation of these dipoles is random, on average, the work function of the polymer as seen by silicon is not affected. However, if the dipoles align preferentially at the interface, the effective work function will be skewed $[67,68]$. This dipole arrangement can be affected by surfactants added to the polymer solution that might aid or impede the rearrangement of the polymer while drying. The terminating species at the Si surface might also be a factor if they preferentially adsorb PEDOT or the dopant species.

Other formulations of interest are the PEDOT/silica composites and Vapour Phase Polymerized (VPP) PEDOT. PEDOT/silica adheres better to the Si substrate than the in situ polymerized version. The required sol-gel mixture is easily prepared by a wet chemical process and can be spin cast on to the substrate $[69,70]$.

PEDOT has been used for quite some time as an electron blocker (hole transport layer) in organic electronics and organic photovoltaics. There have also been some efforts to adapt it for use as a stand-alone electrode [62]. Prior to the initiation of this current study there were no reports found on devices that used an organic conductor to generate a Schottky junction. Since then, reports describing similar devices have appeared at conferences and in publications. Although conversion efficiencies up to $10 \%$ are claimed by other researchers, the behaviour of the organic conductor in these devices is still poorly understood [64,71-73]. As such, a semiconductor solver program was developed as part of this work to better explain the impact of PEDOT on the 


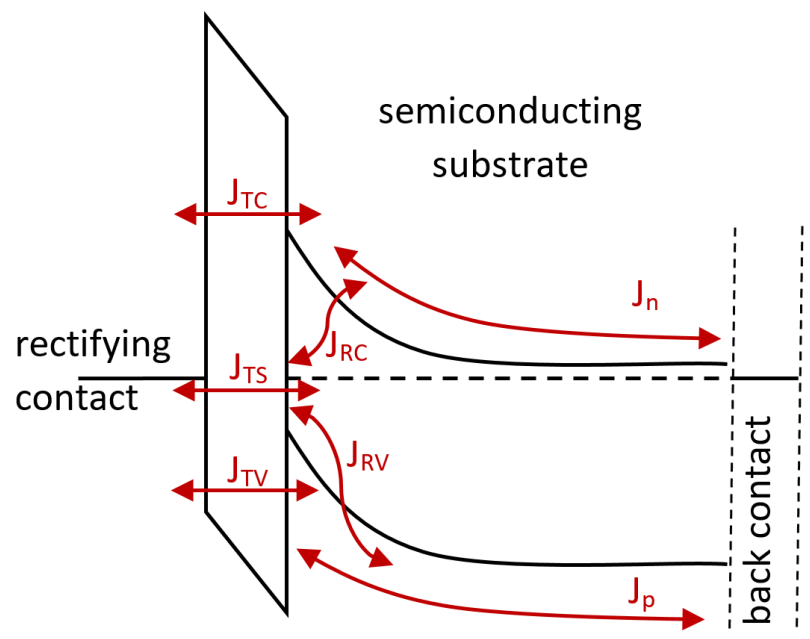

Figure 2.15: Band diagram of an MIS devise showing possible current paths. $J_{T C}$ : metal - conduction band tunneling; $J_{T V}$ : metal - valence band tunneling; $J_{T S}$ : metal - surface states tunneling; $J_{R C}$ : surface states - conduction band recombination; $J_{R V}$ : surface states - valence band recombination; $J_{n} / J_{p}$ : drift diffusion.

structure and the behaviour of devices.

\subsection{Transport Models}

The three distinct areas of the MIS device where the carrier transport needs to be considered are the silicon semiconductor, the insulator, and the organic conductor. The back contact of the device is assumed to be ohmic. The band diagram with the current components for a minority carrier MIS are reproduced in Figure 2.15 for convenience. The current tunnels through the dielectric barrier into the conduction and valence bands and is then conducted in silicon by drift-diffusion. The carriers that tunnel into the surface states in the silicon bandgap first recombine into the silicon bands and then contribute to drift-diffusion as well. 


\subsubsection{Silicon}

The carrier transport in the semiconductor is due to drift and diffusion and can be described by the following set of five coupled non-linear equations. It is convenient to express the electron and hole drift-diffusion current components, $J_{n}$ and $J_{p}$, in terms of the quasi-Fermi levels, $E_{f n}$ and $E_{f p}$ (equations (2.15) and (2.16)). In the case of a static problem, the continuity equations (2.17) and (2.18) can be simplified. The electrical potential is established through the Poisson's equation $(2.19)[8,74]$.

$$
\begin{gathered}
J_{n}=q n \mu_{n} E+q D_{n} \frac{d n}{d x}=\mu_{n} n \frac{d E_{f n}}{d x} \\
J_{p}=q n \mu_{p} E-q D_{p} \frac{d p}{d x}=\mu_{p} p \frac{d E_{f p}}{d x} \\
\frac{\delta n}{\delta t}=\frac{1}{q} \frac{d J_{n}}{d x}+U_{n}=0 \\
\frac{\delta p}{\delta t}=\frac{1}{q} \frac{d J_{p}}{d x}+U_{p}=0 \\
\epsilon \frac{d^{2} V}{d x^{2}}=-q\left(p-n+N_{D}-N_{A}\right)
\end{gathered}
$$

Here $q$ is the electron charge, $n$ and $p$ are electron and hole carrier densities, $\mu_{n}$ and $\mu_{p}$ are electron and hole mobilities, $D_{n}$ and $D_{p}$ are electron and hole diffusion coefficients, $N_{D}$ and $N_{A}$ are the donor and acceptor doping concentrations, and $U_{n}$ and

$U_{p}$ are the electron and hole recombinations rates. In case of simplified Shockley-ReadHall recombination, these two rates are assumed to be equal and defined by equation 2.20, where $\tau_{p}$ and $\tau_{n}$ are carrier lifetimes for holes and electrons respectively [8].

$$
U=\frac{n p-n_{i}^{2}}{\tau_{p}\left(n+n_{i}\right)+\tau_{n}\left(p+n_{i}\right)}
$$

It is typical to solve these equations using the finite difference method. In this case the mesh size must be smaller than the Debye length, $L_{D}$, of the semiconductor 
to be able to resolve the carrier densities correctly [74]. The $L_{D}$ is calculated as:

$$
L_{D}=\sqrt{\frac{\epsilon_{S} k_{B} T}{q^{2} N_{D}}}
$$

Here, $\epsilon_{S}$ is the permittivity of the substrate, $k_{B}$ is the Boltzmann constant, and $T$ is the temperature.

To decouple and solve these equations numerically, the continuity equations are normally discretized using the Scharfetter Gummel method described elsewhere [74].

\subsubsection{Tunneling through Dielectric}

The charge delivered to the silicon/oxide interface is thermionically emitted from the surface and must tunnel through the dielectric. The tunneling currents between the conductor and the silicon conduction and valence bands are defined by equations 2.22 and 2.23 respectively. The density of states for electrons and holes in the conduction and valence bands respectively, $N_{n}$ and $N_{p}$ combined with the occupancy of states in the substrate, relative to the conductor, $f_{s}$ and $f_{m}$ for electrons and $f_{s}^{\prime}$ and $f_{m}^{\prime}$ for holes, define the distribution of carrier densities in energy available for transfer between the substrate and the conductor. The electron and hole velocities, $v_{n}$ and $v_{p}$, combined with the electronic charge, $q$, transform those carrier densities into current densities flowing from the conductor to the silicon conduction and valence bands. To account for the presence of the dielectric a tunneling coefficient, $T_{q}$, is added that attenuates the current density proportionally to the dielectric thickness.

$$
\begin{aligned}
& J_{T C}=\int_{E_{c}}^{\infty} q v_{n}(E) N_{n}(E)\left(f_{s}(E)-f_{m}(E)\right) T_{q}(E) d E \\
& J_{T V}=\int_{-\infty}^{E_{v}} q v_{p}(E) N_{p}(E)\left(f_{s}^{\prime}(E)-f_{m}^{\prime}(E)\right) T_{q}(E) d E
\end{aligned}
$$


The occupancy of states for electrons is defined by the Fermi-Dirac function shown in equation 2.24. The equivalent occupancy of states for holes, $f^{\prime}$, is defined as $f^{\prime}=1$ $f[8]$.

$$
f(E)=\frac{1}{1+e^{\left(E-E_{F}\right) / k T}}
$$

The carrier velocity can be resolved into its components along the three directions as follows $[8]$

$$
v^{2}=v_{x}^{2}+v_{y}^{2}+v_{z}^{2}
$$

Assuming that all of the thermal energy of the free charge carriers is kinetic and is distributed evenly along the three directions, the velocity in the direction perpendicular to the silicon/oxide interface, $\mathrm{v}_{x}$, can be found as shown below:

$$
\begin{array}{r}
E-E_{C}=\frac{1}{2} m^{*} v^{2} \\
E-E_{C}=\frac{1}{2} m^{*} 3 v_{x}^{2} \\
v_{x}=\sqrt{\frac{2}{3 m^{*}}\left(E-E_{C}\right)}
\end{array}
$$

Therefore, the electron and hole velocities of carriers emitted from the silicon surface, $v_{n}$ and $v_{p}$, are

$$
\begin{aligned}
& v_{n}=\sqrt{\frac{2}{3 m_{d e}^{*}}\left(E-E_{C}\right)} \\
& v_{p}=\sqrt{\frac{2}{3 m_{d h}^{*}}\left(E_{V}-E\right)}
\end{aligned}
$$

where $m_{d e}^{*}$ and $m_{d h}^{*}$ are the density of states effective masses for electrons and holes, 
and $E_{C}$ and $E_{V}$ are the edges of the conduction and valence bands in silicon respectively.

When the MIS structure is constructed using a conventional metal, it is assumed that the density of states in the metal is infinite and charge transfer is limited only by the density of states in the semiconductor, $N_{n} / N_{p}$ (and the relative occupancy of states, of course). Here $\hbar$ is the Plank's constant divided by $2 \pi$ :

$$
\begin{aligned}
& N_{n}=N_{C}(E)=\frac{\sqrt{2}}{\pi^{2}} m_{d e}^{3 / 2} \frac{\sqrt{E-E_{C}}}{\hbar^{3}} \\
& N_{p}=N_{V}(E)=\frac{\sqrt{2}}{\pi^{2}} m_{d h}^{3 / 2} \frac{\sqrt{E_{V}-E}}{\hbar^{3}}
\end{aligned}
$$

However, when a polymeric conductor is used instead of the metal, the thin HOMO and LUMO bands as well as the polaron/bipolaron bands can significantly limit the current flow and must be taken into account. The effective density of states must account for both the density of states in the substrate, $N_{C} / N_{V}$, and in the polymer, $N_{\text {poly }, n} / N_{\text {poly }, p}$, and will be limited by the smaller of the two, as follows:

$$
\begin{aligned}
& N_{n}=\frac{N_{C} N_{\text {poly }, n}}{N_{C}+N_{\text {poly }, n}} \\
& N_{p}=\frac{N_{V} N_{\text {poly }, p}}{N_{V}+N_{\text {poly }, p}}
\end{aligned}
$$

To identify the available density of states in the polymeric conductor, the available current paths in the device must be examined. Figure 2.16(b) shows the tunneling current paths for a device with a polymeric conductor. A similar diagram for an MIS device with a conventional metal is shown for comparison in Figure 2.16(a). The LUMO and the upper bipolaron bands $\left(\mathrm{BP}_{1}\right)$ are anti-bonding and, therefore, contain no charge. They might be able to contribute to conduction by accepting charge from 


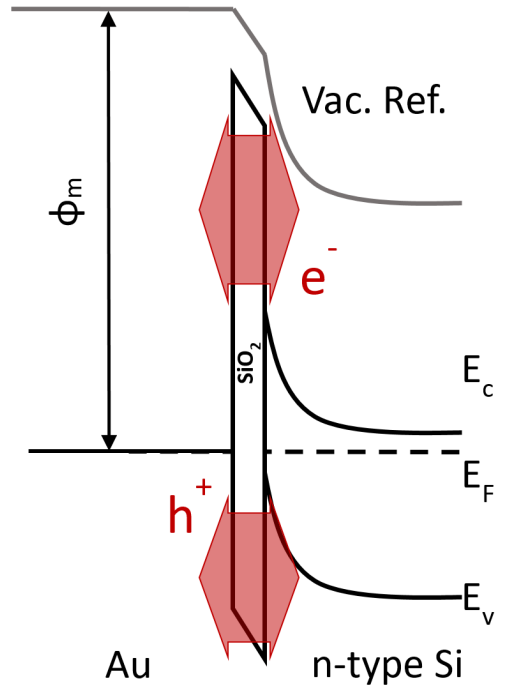

(a) Conventional

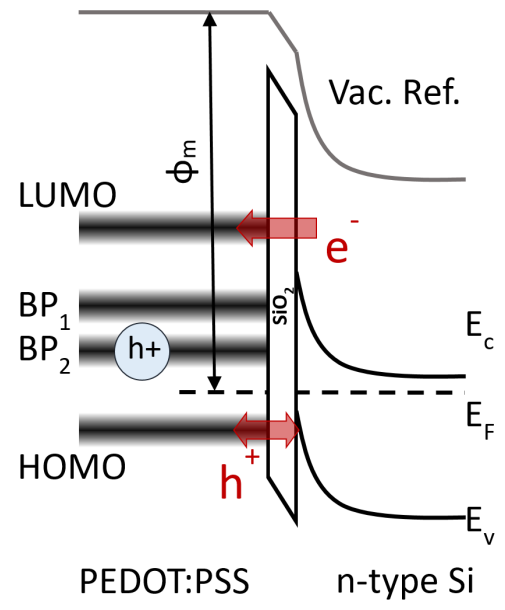

(b) Polymeric

Figure 2.16: Tunneling current paths in (a) conventional MIS device (b) MIS device with a polymeric conductor.

the substrate that would have to be recombined in order to contribute to conduction. It is generally accepted in the literature that the mobile holes in a conductive polymer are located in the lower bipolaron band $\left(\mathrm{BP}_{2}\right)[61]$, therefore, the polymer Fermi level must fall somewhere below it in energy. The HOMO and the lower bipolaron band should be able to accept both electrons and holes depending on the relative alignment of the Fermi levels in the MIS device.

The thin energy bands in the polymer can be modelled as a sum of Gaussians as is often done for hopping mobility calculations (equations 2.33 and 2.34) [61]. For each of the Gaussian energy bands the bandwidth was taken to be six standard deviations, $\sigma . N_{\text {poly0 }}$ is a fitting parameter used to ensure the correct doping density of the polymer. It is determined by setting the integral of the hole density of states modified by the occupation probability to the carrier density in the polymer, $p_{P E D O T}$ $=3 \times 10^{20} / \mathrm{cm}^{3}[62]$. 


$$
\begin{gathered}
N_{\text {poly }, n}=N_{\text {poly } 0}\left(e^{-\frac{\left(E-E_{H O M O}\right)^{2}}{2 \sigma^{2}}}+e^{-\frac{\left(E-E_{B P_{1}}\right)^{2}}{2 \sigma^{2}}}+e^{-\frac{\left(E-E_{\left.B P_{2}\right)^{2}}\right.}{2 \sigma^{2}}}+e^{-\frac{\left(E-E_{L U M O}\right)^{2}}{2 \sigma^{2}}}\right) \\
N_{\text {poly }, p}=N_{\text {poly } 0}\left(e^{-\frac{\left(E-E_{H O M O}\right)^{2}}{2 \sigma^{2}}}+e^{-\frac{\left(E-E_{B P_{1}}\right)^{2}}{2 \sigma^{2}}}\right)
\end{gathered}
$$

Thus established charge available for conduction must still tunnel through the dielectric layer. This process is characterized by the tunneling coefficient, $T_{q}$, derived using the WKB approximation $[17,75,76]$. Though using a triangular barrier would be more accurate, a rectangular barrier was used for simplicity. This approximation is justified as the tunneling barrier is extremely thin (on the order of one to two nanometers) and very high (over three electron volts for $\mathrm{SiO}_{2}$ barrier).

$$
T_{q}=16 \frac{\frac{m_{m}}{k_{m}(E)}\left(\frac{m_{i}}{k_{i}(E)}\right)^{2} \frac{m_{s}}{k_{s}(E)}}{\left[\left(\frac{m_{m}}{k_{m}(E)}\right)^{2}+\left(\frac{m_{i}}{k_{i}(E)}\right)^{2}\right]\left[\left(\frac{m_{i}}{k_{i}(E)}\right)^{2}+\left(\frac{m_{s}}{k_{s}(E)}\right)^{2}\right]} e^{-2 k_{i}(E) t_{i}}
$$

The terms $m_{m}, m_{i}$, and $m_{s}$ are the effective masses of the tunneling charge carriers in the metal, insulator and semiconductor of the MIS device. The $k$ terms are the wavevectors in the same materials, defined by the parabolic model. To get the correct values of tunneling current a dual-band tunneling through the dielectric must be considered, meaning that the charge can tunnel through the barrier formed by the conduction band of the insulator as well as its valence band [77]. And $t_{i}$ is the insulator thickness.

The tunneling current components, $J_{T C}$ and $J_{T V}$, must match the drift-diffusion current components, $J_{n}$ and $J_{p}$, at the semiconductor/insulator interface for a selfconsistent solution of the MIS system: 


$$
\begin{aligned}
& J_{T C}(x=0)=J_{n}(x=0) \\
& J_{T V}(x=0)=J_{p}(x=0)
\end{aligned}
$$

\subsubsection{Polymeric Conductor}

The hopping conduction in the conductive polymer was simply modelled as a potential drop based on the calculated tunneling current and measured PEDOT resistivity:

$$
\Delta V=J \rho_{\text {poly }} t_{\text {poly }}
$$

\subsubsection{Interface States}

The interface states create additional paths for current flow: tunneling between the metal and surface states, $J_{T S}$, as well as generation/recombination between the conduction band and surface states, $J_{R C}$, and generation/recombination between the valence band and surface states, $J_{R V}$. Setting up a tunneling current to the interface states requires defining a Fermi level for the surface states, that determines their

occupancy, $f_{s s}$. This current flow is additionally set up by a time constant that reflects the rate of capture and release of the charge in the surface states, $\tau$, as shown below: [19].

$$
J_{T S}=\int_{E_{V}}^{E_{C}} q N_{s s}\left(f_{s s}-f_{m}\right) T_{q} \frac{1}{\tau} d E
$$

where $N_{s s}$ is the density of the surface states.

The occupancy of the surface states is determined by setting the tunneling current into the surface states equal to the recombination/generation current at the interface. 


$$
J_{T S}=J_{R C}+J_{R V}
$$

Additionally, the drift-diffusion current in the substrate must account for the additional recombination current components. As such, the boundary conditions at the semiconductor/insulator interface (equations (2.36) and (2.37)) are modified to:

$$
\begin{aligned}
& J_{n}=J_{T C}+J_{R C} \\
& J_{p}=J_{T V}+J_{R V}
\end{aligned}
$$

\subsection{Conclusion}

The variety of factors required to design and analyse the performance of photovoltaic devices are diverse and complex. The background information presented here will be the basis for evaluating the various materials, structures, and devices, studied in the course of this work. The relevance of this information to the following chapters is outlined below.

The principles of operation of a metal-insulator-semiconductor diode as a solar cell presented in this overview are applied in this thesis towards an organic hybrid device, which uses a polymeric conductor, poly(3,4-ethylenedioxythiophene) (PEDOT). To this end a variety of PEDOT formulations will be characterized in Chapter 3 and their suitability for photovoltaic application will be assessed.

Substrate nanostructuring for solar cell efficiency improvement will be examined further in Chapter 4. The associated benefits and potential pitfalls will be addressed theoretically and experimentally. 
The idea of a thin energy band structure for organic polymeric conductors, introduced here, will be further explored in Chapters 5 and 6 . Chapter 5 will discuss the mathematical treatment of the thin bands in the context of simulating carrier transport through an MIS structure with a polymeric conductor using the models for carrier transport presented here. Chapter 6 will analyse experimental evidence for such a band structure. 


\section{Chapter 3}

\section{PEDOT}

To optimize the operation of an MIS solar cell, the top electrode must meet a few criteria. It needs to be as transparent as possible to maximize the amount of light that reaches the silicon substrate for photovoltaic conversion. It needs to be as conductive as possible to minimize resistive losses during charge collection. Finally, it needs to have a correct work function to invert the silicon surface to produce a minority

carrier device. For an $n$-type Si substrate doped to $10^{15} \mathrm{~cm}^{-3}$, the work function would ideally exceed $4.9 \mathrm{eV}$.

There is a range of materials used as a top contact in photovoltaics. Among them are indium tin oxide (ITO), poly(3,4-ethylenedioxythiophene) (PEDOT), polyaniline (PANI), polypyrrole, and graphene [63]. Their relevant properties are summarized in Table 3.1. All of these materials require finding a balance between conductivity and transparency. While, it is hard to compete with ITO having conductivities on the order of $3000 \mathrm{~S} / \mathrm{cm}$ with the corresponding transparency of $80 \%$, its work function is reported to be in the $4.6 \mathrm{eV}-4.8 \mathrm{eV}$ range. The latter is not ideal for achieving strong inversion at the Si surface, but it can be increased with post-processing $[63,78$, 79]. Additionally, the uncertain supply of indium makes this material expensive and undesirable. Other, non-indium-containing, transparent conductive oxides (TCOs) have been a subject of recent research, and many of them have sufficiently high 


\begin{tabular}{c|c|c|c|c} 
Material & Transparency & $\sigma[\mathrm{S} / \mathrm{cm}]$ & $\phi_{m}[\mathrm{eV}]$ & Reference \\
\hline \hline ITO & $>80 \%$ & $2500-5000$ & $4.6-4.8$ & {$[63,78,79]$} \\
polyanaline & $>60 \%$ & up to 50 & 5.27 & {$[82-84]$} \\
polypyrrole & $>40 \%$ & $10^{-3}$ & $5-5.1$ & {$[85-88]$} \\
graphene & $>85 \%$ & $>1000$ & $4.5-4.7$ & {$[89-94]$} \\
PEDOT & $>70 \%$ & up to 1000 & $4.2-5.2$ & {$[63,66,95,96]$}
\end{tabular}

Table 3.1: Comparison of transparent conductors relevant to choosing the top electrode for a minority-carrier MIS device.

work functions. However, good $p$-type TCOs are very difficult to produce. ${ }^{1}$ [81] PANI has a sufficiently large work function of approximately $5.3 \mathrm{eV}$, but has lower transparency [82-84]. Polypyrrole has a high work function, but poor conductivity and transmission [85-88]. Intrinsic graphene has a lower-than-desired work function in the range of $4.5 \mathrm{eV}-4.7 \mathrm{eV}$, but it can be increased to $5.1 \mathrm{eV}$ by doping. It can have conductivities over $10 \mathrm{~S} / \mathrm{cm}$ with corresponding transparencies of approximately 85\% [89-93]. In 2013 graphene films with conductivity up to $1240 \mathrm{~S} / \mathrm{cm}$ have been demonstrated, however their growth requires temperatures in excess of $950^{\circ} \mathrm{C}$ [94]. PEDOT, on the other hand is a mature technology, developed as an alternative to ITO. It boasts conductivities up to $1000 \mathrm{~S} / \mathrm{cm}$ with a corresponding transparency of more than $70 \%$ in the optical range $[63,66,95,96]$. Given the maturity of the product, its commercial availability and favourable properties for a top electrode, it was an obvious choice for the MIS device used in this study.

PEDOT is available in many formulations. It can be polymerized in situ from commercially available ethylenedioxythiophene (EDOT) monomers [97].

\footnotetext{
${ }^{1}$ It is theoretically possible to produce an MIS device on a $p$-type substrate with an $n$-type transparent oxide, but TCOs tend to have too high of a work function to generate a minority carrier device. However, a surface modification scheme has been reported that can reduce the work function of virtually any film. It uses a thin layer of polymers containing aliphatic amine groups as surface modifiers to lower the work function of a surface by as much as $1 \mathrm{eV}[80]$ Such a process might make this scheme possible.
} 
More conveniently, it can be dispersed from an aqueous solution, doped with poly(styrenesulfonate) (PSS). A wide variety of PEDOT:PSS formulations are commercially available with various viscosities, particle sizes, and conductivities [66,98].

The following sections describe the characterization of various formulations of PEDOT and assessment of their suitability for photovoltaic applications. PEDOT:PSS was found to be a good candidate for hybrid MIS diode formation on flat substrates. However, it shows poor penetration into a nanostructured surface. In situ PEDOT suffers from poor adhesion to the Si surface. To mitigate these problems a PEDOT/silica composite was also explored.

\subsection{PEDOT:PSS}

The convenience of PEDOT:PSS makes it an ideal starting point for development of a hybrid solar cell. It has excellent adhesion to silicon and silicon dioxide, potentially high conductivity up to $1000 \mathrm{~S} / \mathrm{cm}$, and the optimal work function of $5.1 \mathrm{eV}-5.2 \mathrm{eV}$ for creating a minority carrier diode on an $n$-type substrate.

Like many conducting materials, PEDOT:PSS has a trade-off between transparency and conductivity. The thinner the layer of PEDOT:PSS being used, the more transparent it is. However, conductance decreases with thickness. This tradeoff places a limitation on the amount of electrical power that can be extracted from a photovoltaic device, as a compromise needs to be reached between the loss of optical power reaching the cell due to a finite transparency of PEDOT:PSS and the loss of the generated electrical power during charge collection due to the resistance of the film.

A variety of PEDOT:PSS formulations was examined: Baytron P Al 4083, Clevios FET, Clevios PH750, Clevios PH1000. Baytron P Al 4083, designed as an extremely 
thin electron blocking layer for organic electronics, was transparent enough for acceptable light penetration to the substrates only in very thin layers. However, in such thin layers it lacked a good conductivity to support efficient charge collection.

The Clevios brand materials were supplied as experimental samples, while these formulations were still in development by the company. Despite excellent transmission and conductivity properties, the use of Clevios FET (designed as a transparent electrode) produced devices with no photoresponce. No explanation for such behaviour was found, but clearly this formulation is of no use in a photovoltaic application, unless this issue can be understood and corrected.

The best solar cell performance was achieved with Clevios PH750, a PEDOT:PSS formulation specifically designed for high conductivity and high transparency, claiming a maximum achievable conductivity of $750 \mathrm{~S} / \mathrm{cm}$. This product was used for the majority of experiments, until it was superseded by the Clevios PH1000 formulation with a maximum conductivity of $1000 \mathrm{~S} / \mathrm{cm}$. To achieve such high conductivities, the alignment of the PEDOT polymer chains must be manipulated. In solid-state conductors, the conduction band is continuous throughout the material. In polymeric conductors like PEDOT:PSS, the conduction bands span only the individual conjugated polymers. As a result, the conductivity is limited by the thermally-activated transition of charge between polymer chains, termed "hopping". The activation energy for this transition can be lowered by strategically stacking polymer chains. It is well established that an addition of a high boiling point solvent or surfactant results in better PEDOT alignment and conductivity $[95,99,100]$. This addition of a surfactant is used in the Clevios high conductivity polymers. However, the surfactant is not pre-mixed in the formulation and must be added during processing. The manufacturer suggests using dimethyl sulfoxide (DMSO) [101]. The best experimental conductivity achieved was $510 \mathrm{~S} / \mathrm{cm}$. This is a reasonable value to achieve for PH1000 as the conductivity of this PEDOT formulation was shown to vary from 
$1 \mathrm{~S} / \mathrm{cm}$ to over $1000 \mathrm{~S} / \mathrm{cm}$ depending on the amount of surfactant used as well as ambient and deposition conditions, such as air temperature, humidity, spin speed, time and acceleration, that can all influence polymer alignment [95, 99, 102-104].

\subsubsection{Deposition}

On a flat substrate, the deposition procedure is very straightforward. The full deposition procedure based on the discussion with the supplier [101] is given below:

1. Combine Clevios PH750 + 5\% DMSO by weight.

2. Stir the mixture at $50^{\circ} \mathrm{C}$ for $10 \mathrm{~min}$ at $330 \mathrm{RPM}$.

3. Drip the mixture over the entire silicon wafer through a $0.45 \mu \mathrm{m}$ filter.

4. Spin-on at $1000 \mathrm{RPM}$ for $30 \mathrm{~s}$ with acceleration of $180 \mathrm{RPM} / \mathrm{s}$.

5. Bake at $130^{\circ} \mathrm{C}$ for $10 \mathrm{~min}$ on a hotplate.

6. Bake at $200^{\circ} \mathrm{C}$ for $10 \mathrm{~min}$ on a hotplate.

\subsubsection{Characterization}

From the capacitance-voltage (CV) measurements shown in Figure 3.3, the work function of Clevios PH750 was found to be in excellent agreement with work functions reported for PEDOT:PSS with $\phi_{P H 750}=5.14 \pm 0.02 \mathrm{eV}$. The reported uncertainty generously accounts for the barrier-lowering effect. See Appendix A for relevant data and work function extraction method.

The thickness of this produced PEDOT:PSS film was measured with a WYKO optical profilometer to be $340 \mathrm{~nm}$. For such a relatively thick film, the transparency was excellent, exceeding $80 \%$ over the optical range of $400 \mathrm{~nm}$ to $800 \mathrm{~nm}$ as shown in Figure 3.1. The conductivity, however, was not as high as advertised, but was 


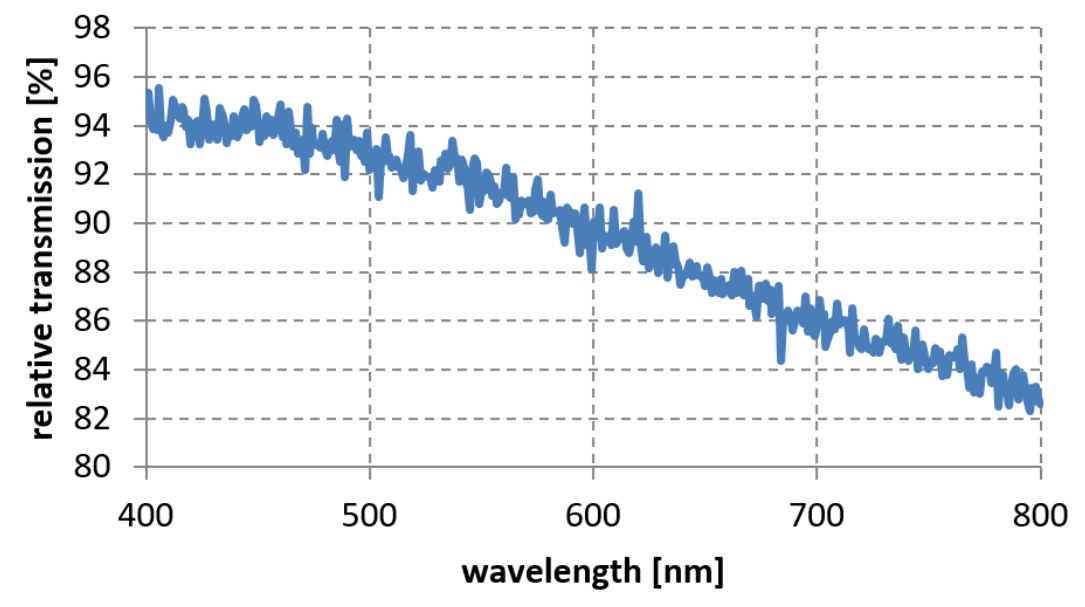

Figure 3.1: Transmission of $340 \mathrm{~nm}$ of dry Clevios PH750 PEDOT:PSS film relative to a clean glass substrate.

measured to be $170 \mathrm{~S} / \mathrm{cm}$. The discrepancy is likely due to non-optimal drying conditions as well as the resultant thickness of the film.

\subsubsection{Devices}

The proof-of-concept MIS devices where constructed on flat wafers with a structure shown in Figure 3.2.

The front sides of the wafers were exposed to hydrofluoric acid (HF) vapour to remove any native oxide. After the HF treatment, the wafers were immediately placed in an oxidation furnace for $10 \mathrm{~min}$ at various temperatures $\left(450^{\circ} \mathrm{C}-550^{\circ} \mathrm{C}\right)$ to grow increasing thicknesses of $\mathrm{SiO}_{2}$. Oxidation was followed by deposition of backside aluminum by e-beam evaporation. PEDOT:PSS (Clevios PH750 supplied by H.C. Starck) was spin-cast on top of the grown oxide. The structure was completed by screen printing a silver ink (Dupont 5028) finger grid. The wafers were stored in a dry nitrogen box or under vacuum between processing and evaluation to avoid undesirable continued growth of the oxide layer. Most importantly this precaution ensured that the reference sample remained without interfacial oxide. 


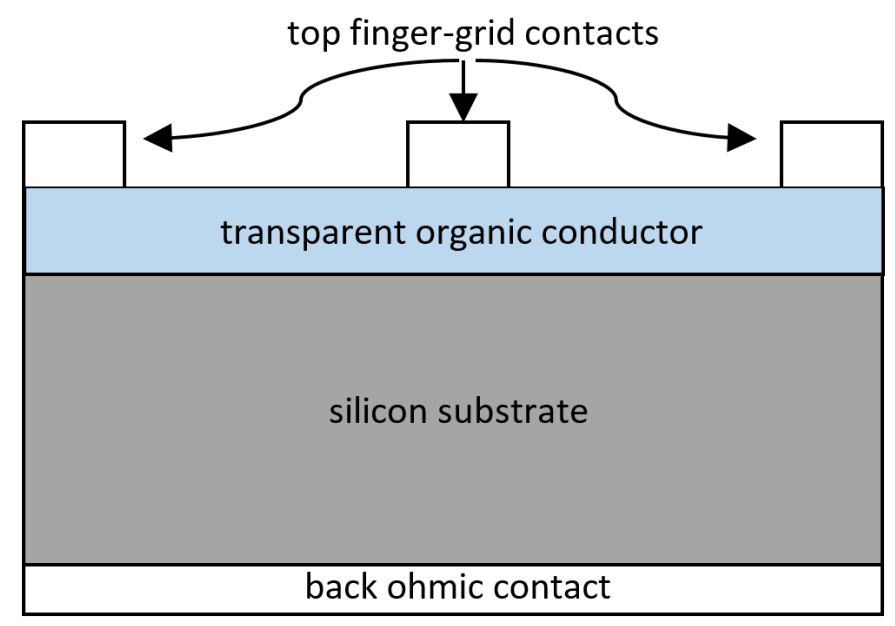

Figure 3.2: Desired structure for experimental hybrid MIS diodes.

\begin{tabular}{c|c|c} 
Oxidation Temperature $\left[{ }^{\circ} \mathrm{C}\right]$ & Oxide Thickness $[\mathrm{nm}]$ & Effective Work Function $[\mathrm{eV}]$ \\
\hline \hline No oxidation & 0 & 5.05 \\
450 & $0.57 \pm 0.01$ & 5.06 \\
500 & $0.79 \pm 0.01$ & 5.07 \\
550 & $2.41 \pm 0.02$ & 5.14
\end{tabular}

Table 3.2: The extracted oxide thicknesses and effective work functions of MIS devices on $n$-type silicon with PEDOT:PSS.

The resultant oxide thicknesses, reported in Table 3.2 were measured with a Plasmos $632 \mathrm{~nm}$ multiangle ellipsometer (model SD2000). The produced insulator thicknesses are in the desired range and increase with oxidation temperature.

The capacitance-voltage measurements of these devices, shown in Figure 3.3, display an increase in the effective conductor work function (reported in Table 3.2), which would normally be consistent with an increasing oxide thickness in a typical MOS capacitor. However, in this case the oxide thicknesses are too small to justify the observed increase of $0.09 \mathrm{eV}$. Thus it is postulated that the low-temperature oxidation results in an incomplete passivation of the interface states on the silicon surface, with progressively better passivation at higher oxidation temperatures. As the surface interface charge is reduced, the CV curve shifts to higher voltages. 


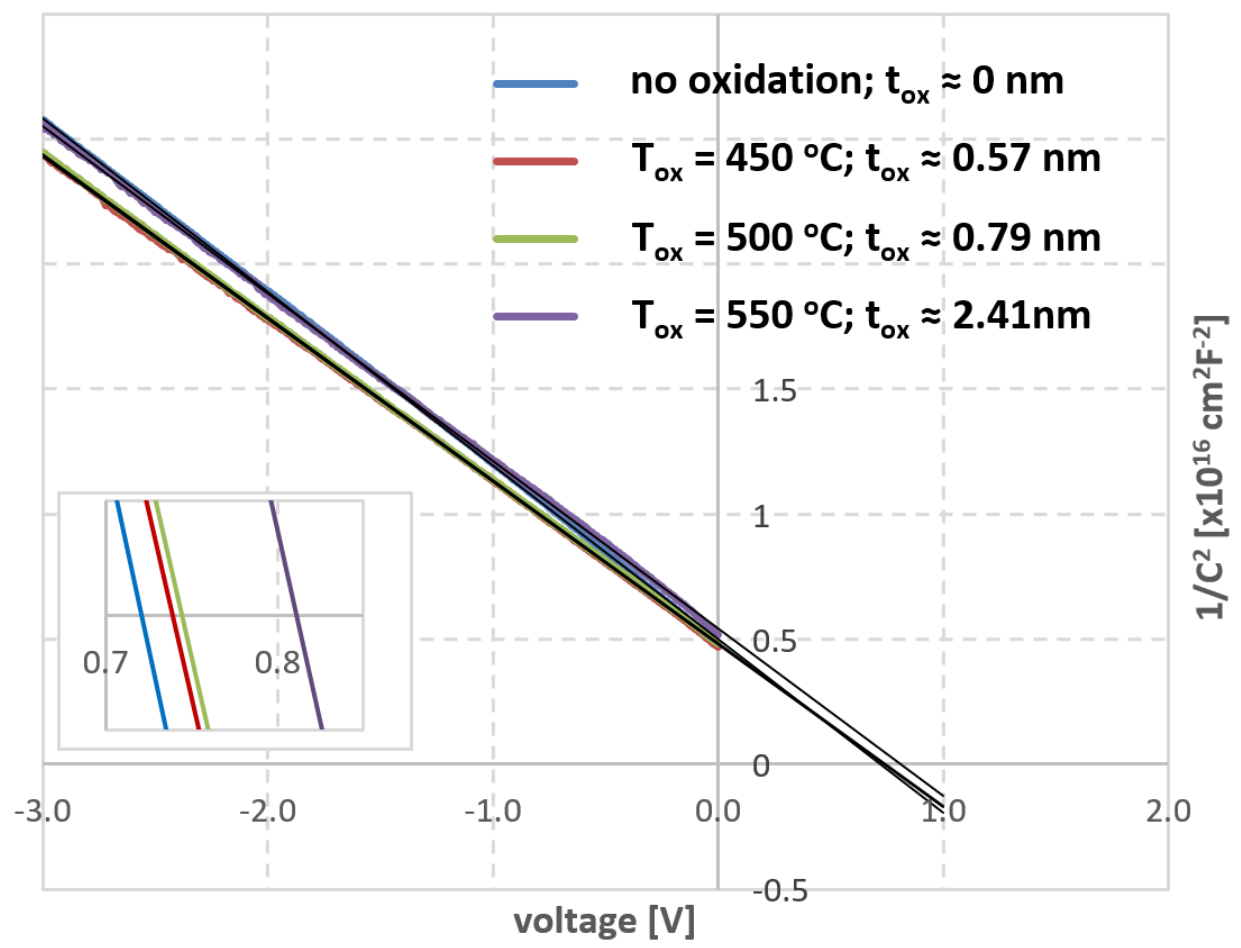

Figure 3.3: Capacitance-voltage (CV) characteristics of MIS devices on $n$-type silicon with thermally-grown oxide and PEDOT:PSS conductor. The measurements are normalized to the active device area extracted from the CV measurements. The inset zooms in on the voltage intercepts of the presented linear fits, from which the PEDOT:PSS work function is calculated. 


\begin{tabular}{c|c|c} 
Oxidation Temperature $\left[{ }^{\circ} \mathrm{C}\right]$ & $N_{D}$ from 4-point probe $\left[\mathrm{cm}^{-3}\right]$ & $N_{D}$ from CV $\left[\mathrm{cm}^{-3}\right]$ \\
\hline \hline No oxidation & $1.73 \times 10^{15}$ & $9.54 \times 10^{8}$ \\
450 & $1.85 \times 10^{15}$ & $1.63 \times 10^{8}$ \\
500 & $1.85 \times 10^{15}$ & $7.01 \times 10^{7}$ \\
550 & $1.80 \times 10^{15}$ & $2.74 \times 10^{8}$
\end{tabular}

Table 3.3: Substrate doping densities measured with a four-point probe as well as extracted from the CV measurements of the hybrid diodes made with PEDOT:PSS PH750.

There was a discrepancy observed in the substrate doping, with doping densities extracted from the CV measurement being orders of magnitude lower then the values measured with a four-point probe prior to device fabrication as summarized in Table 3.3. The discrepancy is postulated to be due to conductance issues associated with the measurements.

Figure 3.4 shows various aspects of the MIS devices made with PEDOT:PSS PH750. Subplots 3.4(a) through 3.4(c) show the dark current density, while subplot 3.4(d) shows the device behaviour under halogen lamp illumination. Subplot 3.4(a) shows the reverse bias current, which does not saturate. Such behaviour is expected in Schottky and MIS diodes due to the image force barrier lowering that has an increasing effect in reverse bias [8]. Also as is predicted, and desired, the reverse-bias current decreases with increasing oxide thickness. This should lead to an increase in the open circuit voltage. However, in the -1 to $-1.5 \mathrm{~V}$ range the reverse-bias current of the device with $t_{o x}=2.41 \mathrm{~nm}$ exceeds that of the device with $t_{o x}=0.57 \mathrm{~nm}$. Such behaviour was explained by Card and Rhoderick as the result of a better alignment of the metal Fermi level with the conduction band of silicon due to the higher mobility in energy allowed by the thicker oxide (since the oxide can support a larger potential drop across itself). Card's explanation is illustrated in Figure 3.5 along with a sample of his data [105]. 


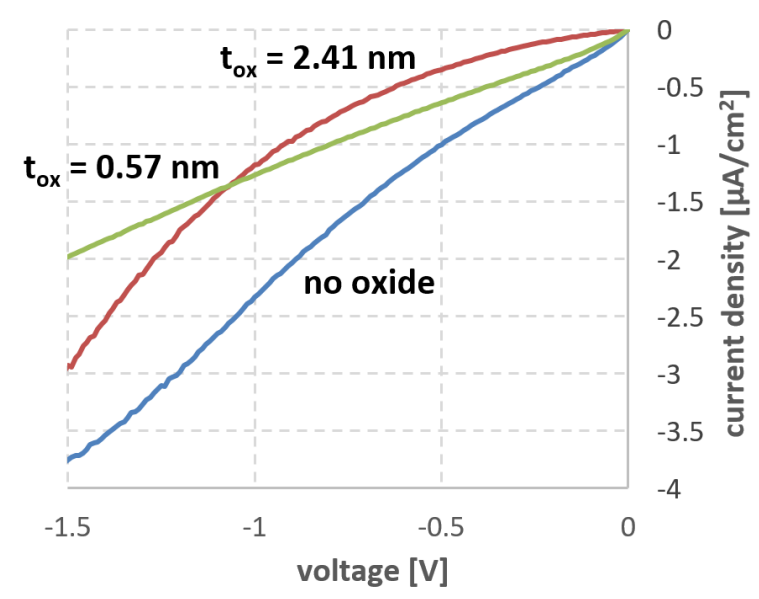

(a)

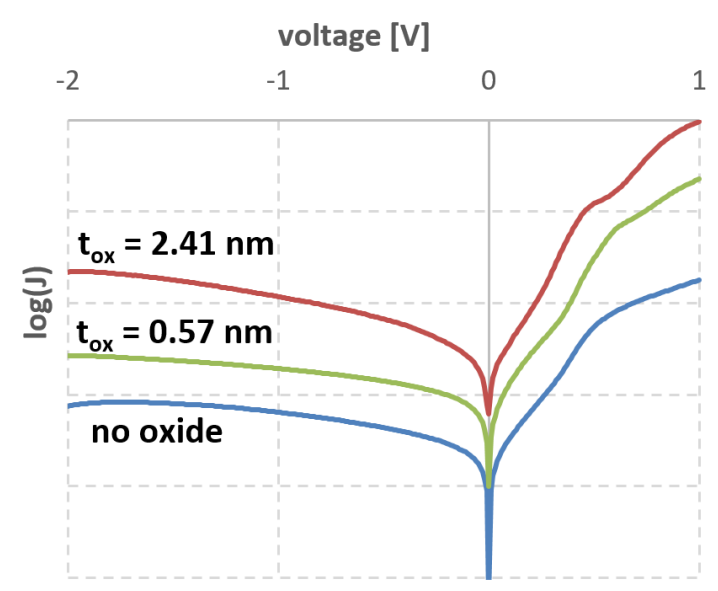

(c)

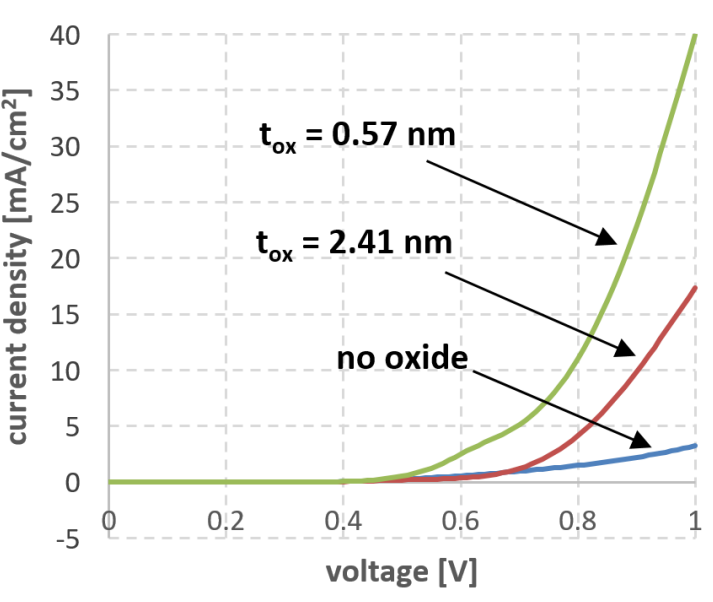

(b)

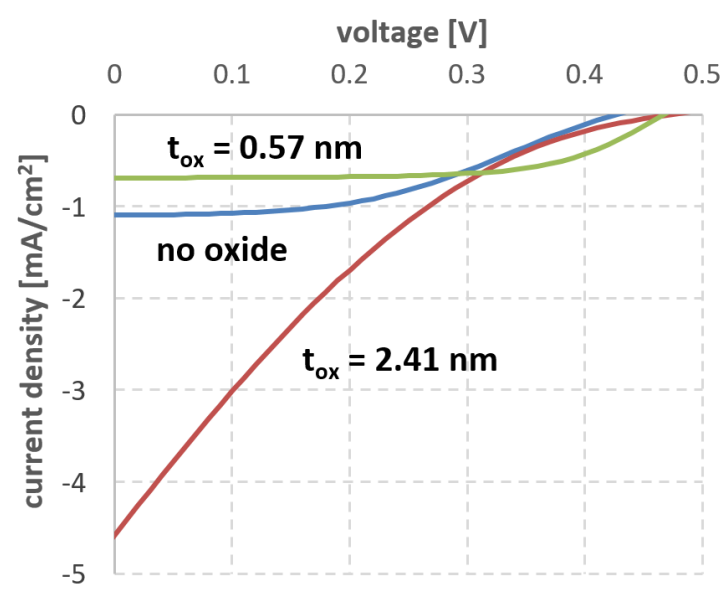

(d)

Figure 3.4: Current-voltage (IV) characteristics of MIS devices on $n$-type silicon with thermally-grown oxide and PEDOT:PSS conductor. (a) Dark reverse bias current (b) Dark forward bias current (c) Dark current-voltage characteristic on a logarithmic scale with the curves offset from one another for better visibility (d) Photoresponce under halogen light illumination. 


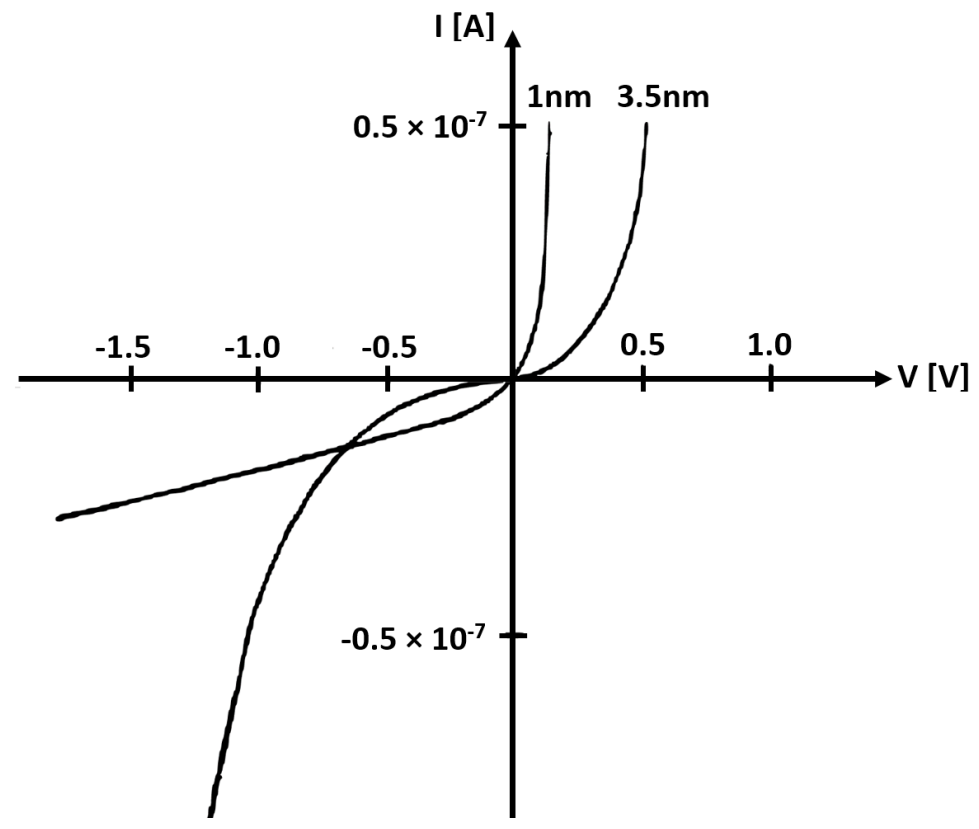

(a)

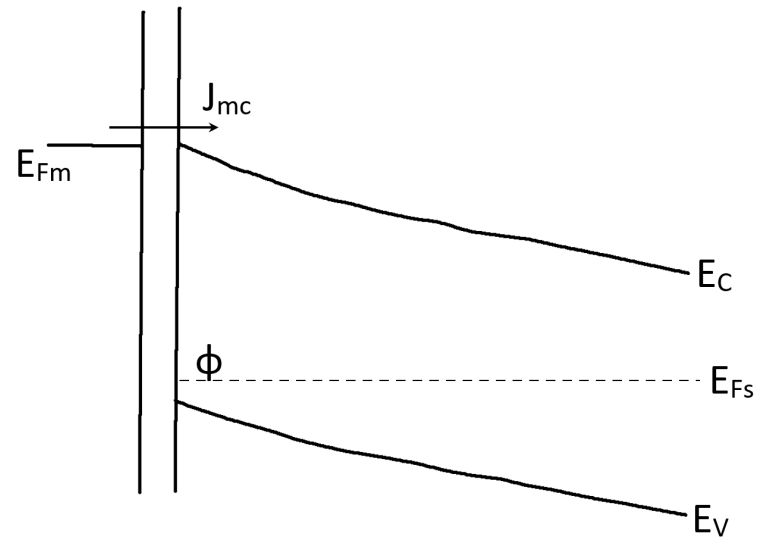

(b)

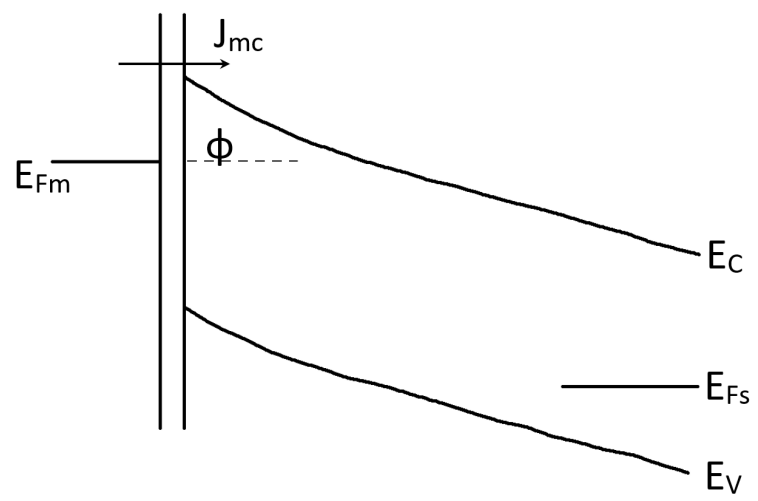

(c)

Figure 3.5: Explanation given by Card and Rhoderick for a higher reverse bias current density over a range of biases for an MIS diode with a thicker tunneling oxide (a) Card's data for two MIS diodes with different oxide thicknesses (b) thick oxide interfacial layer allows the metal Fermi level to better align with the conduction band; higher availability of electrons in the metal at the band edge overcompensates for the thicker tunneling barrier (c) thin oxide does not support as much of a voltage drop across it and the metal Fermi level cannot pull up to the conduction band edge. After [105]. 
Figure 3.4(b) shows the dark forward-bias current. As expected, the device with a thinner oxide supports higher current. However the device with no intentional oxide performs very poorly. This loss in current is most likely due to surface recombination at interface states, which would have been passivated in the oxidised devices.

Plotting the current on a logarithmic scale (Figure 3.4(c)) reveals an interesting feature at around $0.5 \mathrm{~V}$. It is prominent in the oxidised devices, but is very slight in the reference device. The presence of interface states would account for the obscuring of this feature in the unoxidized device. This feature may be manifestation of the finite width of the energy bands in PEDOT:PSS and will be discussed further in Chapter 6 .

Finally, Figure 3.4(d) shows the current density under illumination. The short circuit currents here are well below the $33 \mathrm{~mA} / \mathrm{cm}^{2}$ that are commonly discussed in connection to solar cells. However, the light source here is a halogen light, which would not be as bright as the sun, nor have the same spectrum. Thus the nature of the source used is, at least in part, responsible for the low short circuit currents. The open circuit voltages are around $0.47 \mathrm{~V}$, which might be low compared to some reported values, but are consistent with the short circuit currents, and can be expected to increase with increased illumination. The wild variability of the short circuit current is most likely due to the poor alignment of the light source with the device during measurement, which makes the efficiency hard to estimate well. The device with the thickest oxide displays signs of both high series resistance and low shunt resistance. The high series resistance can be easily explained by the presence of such a thick tunneling oxide. The shunt resistance may be due to a probe puncturing the metal contact, the PEDOT layer and potentially damaging the oxide. This problem has been observed throughout many experiments in the course of this work.

A device with no intentional oxide was cleaved from the substrate and measured under a solar simulator with 0.8 sun illumination. The resultant current-voltage 


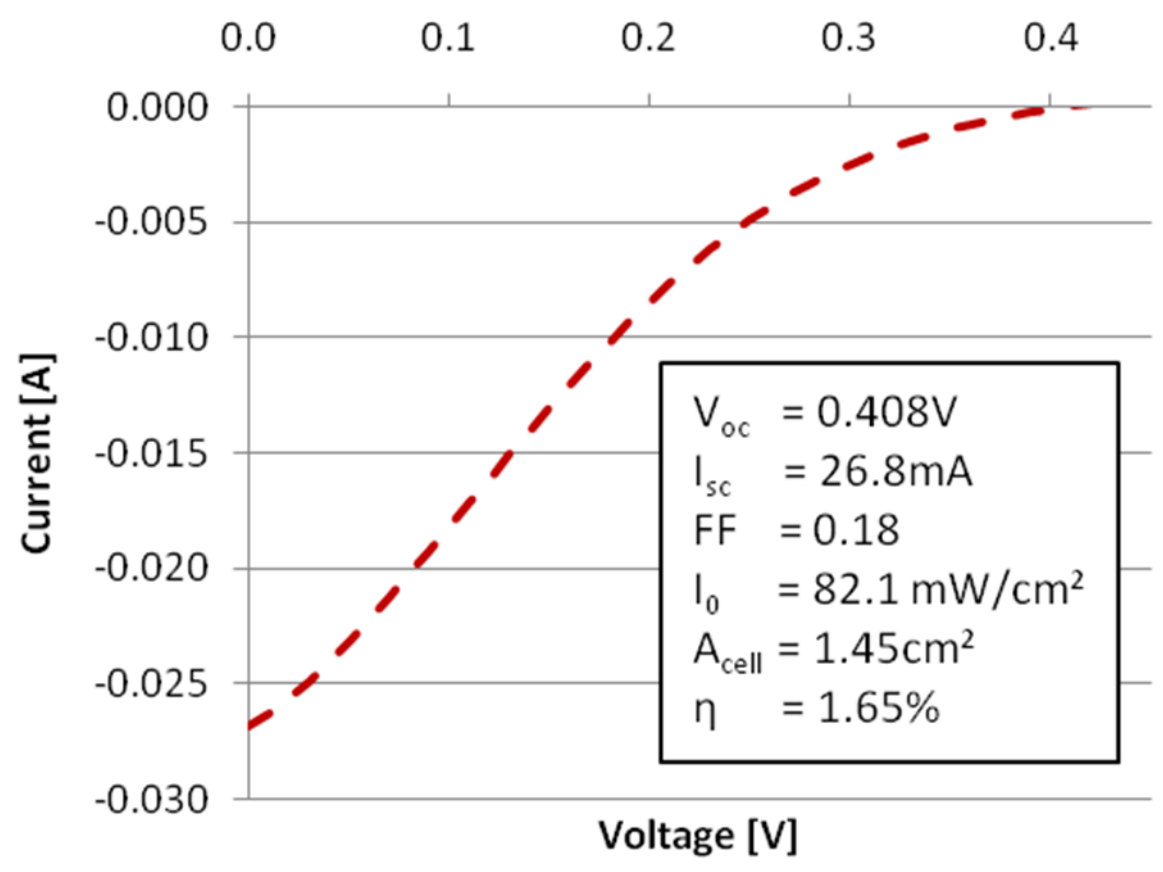

Figure 3.6: Current-voltage characteristics of a flat diode on $n$-type silicon with no intentional interfacial oxide and PEDOT:PSS conductor. The measurements are performed in a solar simulator with 0.8 sun illumination.

characteristics are shown in Figure 3.6. Despite a poor fill-factor, likely due to surface state recombination, the device exhibited $1.65 \%$ conversion efficiency. Using this data in combination with current-voltage characteristics of oxidised devices from this series of experiments, a potential photovoltaic efficiency of an optimized organic hybrid MIS diode was estimated at nearly $12 \%[71]$.

\subsubsection{Compatibility with Nanostructures}

As devices on nanostructured surfaces are desired, the penetration of PEDOT:PSS into a high-aspect ratio structure was tested. The full details of the deposition technique will be discussed in Chapter 4. However, the penetration into nanostructure was observed to be quite poor as can be seen in the SEM image in Figure 3.7. 


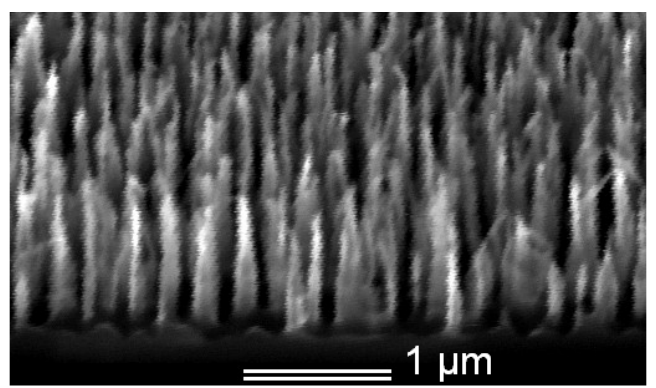

(a)

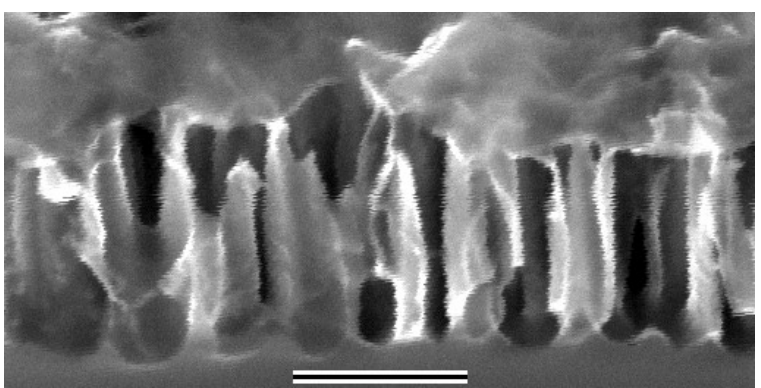

(b)

Figure 3.7: Scanning electron microscope (SEM) image of reactive ion etched silicon grass (a) as fabricated (b) with vacuum-deposited Clevios PH750. The details of the deposition process are explained in Chapter 4 . The scale bar is $1 \mu \mathrm{m}$.

\subsection{In situ PEDOT}

A solar cell with a nanostructured surface for reflection minimization was to be explored as part of this thesis. As such, penetration of the conductive polymer into the structure was identified as a potential issue. As the smallest component of the polymer chains, the EDOT monomer has the best chance of reaching the bottom of the nanostructure. As such, in situ polymerization was a logical approach. The full deposition procedure is detailed in Section 3.2.1.

\subsubsection{Deposition}

The Clevios website suggests depositing a thin adhesion layer of PEDOT:PSS prior to the in situ deposition, since in situ PEDOT does not adhere to silicon very well and may delaminate from the surface [97]. However, this approach would defeat the purpose of using the monomers. As such the adhesion layer was omitted. Additionally, it is recommended that drying the film be done at humidity levels of 30\%-70\%. In fact, the conductivity seems to be optimum when drying PEDOT at 30\%-40\% humidity $[62,97]$. Unfortunately, a humidity controlled environment was not available for this research and as such the drying was performed at the ambient humidity level of about 
20\%-30\%. The full deposition procedure is given below:

1. Dissolve $0.75 \mathrm{~g}$ of imidazole in $30 \mathrm{~g}$ of Fe(III)tosylate in butanol (Baytron CB40, now sold under the name Clevios CB 40 V2).

2. Add $1.5 \mathrm{~g}$ EDOT monomer (Baytron $\mathrm{M}$, now sold under the name Clevios M).

3. Spin the solution onto a flat substrate:

(a) Drip solution over the entire substrate through a $0.45 \mu \mathrm{m}$ filter.

(b) Spin at $1000 \mathrm{RPM}$ for $30 \mathrm{~s}$ with acceleration of $180 \mathrm{RPM} / \mathrm{s}$; lid closed.

4. Dry on a hotplate at $130^{\circ} \mathrm{C}$ for $1 \mathrm{~h}$.

5. Rinse sample by agitating in a beaker of n-butanol followed by deionized water rinse to remove residual oxidants.

6. Dry with $\mathrm{N}_{2}$ flow.

\subsubsection{Characterization}

The work function for in situ PEDOT reported in the literature ranges from $4.25 \mathrm{eV}$ to $5.4 \mathrm{eV}$ and seems to vary depending on the oxidation species used and the doping level obtained. It is also important to note that these work function values were obtained with surface measurement techniques, such as UV photoelectron spectroscopy (UPS) and Kelvin probe [67,106-108]. In case of devices examined in this thesis, however, the work function at the PEDOT/silicon interface is of greater importance. It can be expected to vary from the reported values due to the influence of the silicon surface conditions at the time of PEDOT deposition. The deposition conditions themselves, such as substrate temperature and ambient humidity can influence the level of PEDOT doping, affecting its work function [107]. Therefore, even though 


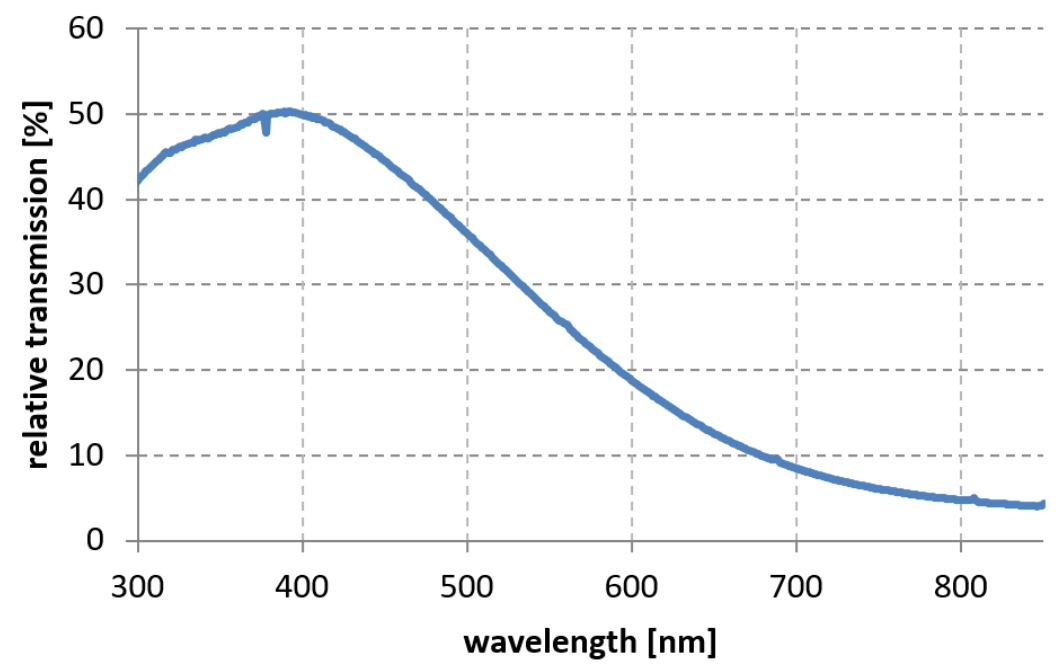

Figure 3.8: Transmission spectrum of $306 \mathrm{~nm}$ thick in situ PEDOT.

the work function of $\phi_{m}=5.1 \mathrm{eV}$ extracted from the measured CV characteristics is higher than $\phi_{m}=4.4 \mathrm{eV}$ reported for in situ PEDOT doped with tosylate [108], as is done in this work, the measured work function will be used.

The resultant conductive film was measured to be $310 \pm 50 \mathrm{~nm}$ thick based on SEM images taken in several locations. The average sheet resistance was determined by a simple two-point measurement as suggested by Clevios [65] and was found to be $55 \pm 5 \Omega / \square$. This gives a conductivity of $\sigma=600 \pm 100 \mathrm{~S} / \mathrm{cm}$.

While the conductivity is very favourable, the film is too thick to give good transmittance. The transmittance was measured with a PerkinElmer Lambda 900 spectrometer and calculated relative to the glass substrate. The full spectrum in the optical range can be seen in Figure 3.8. The irregularities in the spectrum at $380 \mathrm{~nm}$, $560 \mathrm{~nm}, 690 \mathrm{~nm}$, and $810 \mathrm{~nm}$ are measurement artifacts. The artifact at $380 \mathrm{~nm}$ is the most prominent, with others becoming visible at lower light intensities.

The adhesion of in situ PEDOT to the silicon surface, however, was poor as expected. It failed the Scotch tape test where a piece of sticky tape is applied to the film and then peeled off at $45^{\circ}$ to the surface, indicating unacceptably poor 


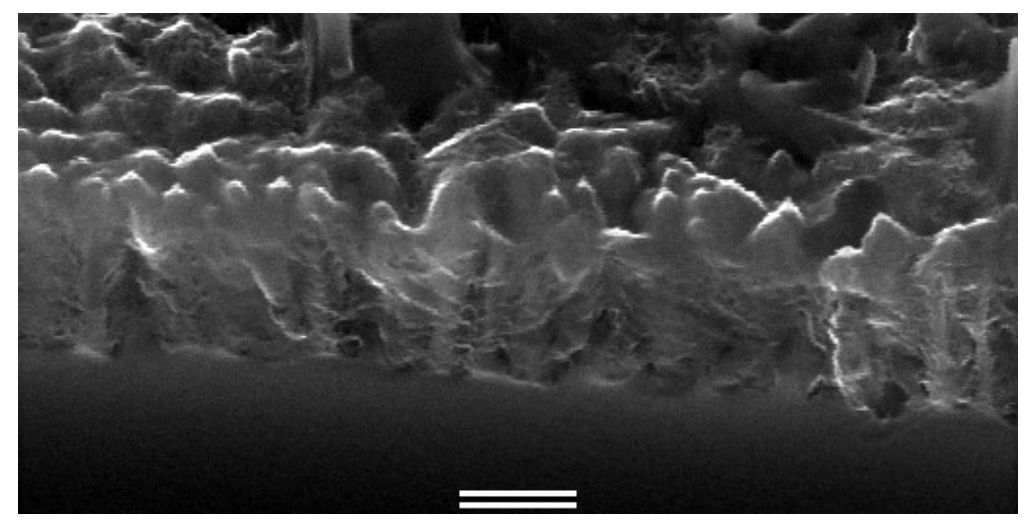

Figure 3.9: SEM image of reactive-ion-etched silicon grass with in situ deposited PEDOT.

adhesion [109].

\subsubsection{Compatibility with Nanostructures}

The penetration of in situ deposited PEDOT into a nanostructure was found to be excellent as seen in Figure 3.9, with the best results achieved by depositing the monomer first, followed by the oxidant and surfactant mixture. However, such a procedure makes the monomer/oxidant ratio hard to control precisely. This could result in considerable variability of the resultant PEDOT film. The full details of the deposition technique will be discussed in Chapter 4 .

Another concern was the transparency of the film. If PEDOT was to be used to fill in the surface nanostructure entirely, the required film thickness of $>1000 \mathrm{~nm}$ would reduce transparency to unacceptable levels. A potential solution to this problem will be discussed in Chapter 4 . 


\subsection{PEDOT/Silica Composite}

To resolve the adhesion problem, a PEDOT/silica composite was investigated. It uses a sol-gel prepared silica matrix in which to suspend in situ polymerized PEDOT. Obtaining sol-gel silica is a fairly simple process of hydrolysis of tetraethyl orthosilicate (TEOS). With all the components being liquid, including the EDOT monomers and the oxidising agent, the composite is easily assembled in a single container and can be spin-deposited on a substrate. Once the mixture is dispersed, the oxidation of the polymer and the gelation of the silicate is allowed to finish on the substrate $[80,110]$. The composite was assumed to be a two-phase heterogeneous mixture of its constituents and the morphology was not investigated. In this material PEDOT provides the conductivity, while the silica ensures excellent adhesion to the silicon surface. Addition of silica also provides an extra degree of freedom for achieving the best conductivity/transparency balance.

\subsubsection{Deposition}

The deposition procedure is given below for the best performing formulation of the composite.

1. Prepare the silica sol-gel.

(a) Dissolve $5 \mathrm{~g}$ of tetraethylorthosilicate (TEOS) in $2 \mathrm{~g}$ of $\mathrm{H}_{2} \mathrm{O}$ and $43 \mathrm{~g}$ of isopropanol (IPA).

(b) Add nitric acid as a hydrolysis catalyst to establish a $\mathrm{pH}$ of 1.5 .

\section{Prepare PEDOT.}

(a) Dissolve $0.75 \mathrm{~g}$ of imidazole in $30 \mathrm{~g}$ of Fe(III)tosylate in butanol (Baytron CB40, now sold under the name Clevios CB 40 V2). 
(b) Add $1.5 \mathrm{~g}$ EDOT monomer (Baytron M, now sold under the name Clevios M)

3. Add PEDOT to sol-gel.

(a) Filter the PEDOT mixture through a $0.45 \mu \mathrm{m}$ syringe filter into the sol-gel.

4. Spin the solution onto the substrate:

(a) Drip solution over the entire substrate through a $0.45 \mu \mathrm{m}$ filter.

(b) Spin at $1000 \mathrm{RPM}$ for $30 \mathrm{~s}$ with acceleration of $180 \mathrm{RPM} / \mathrm{s}$ (setting 2); lid closed.

5. Dry on a hotplate at $130^{\circ} \mathrm{C}$ for $1 \mathrm{~h}$.

6. Rinse sample by agitating in a beaker of n-butanol followed by deionized water rinse to remove residual oxidants.

7. Dry with $\mathrm{N}_{2}$ flow.

In the investigation of a PEDOT/silica composite, a variety of ratios of constituent materials were used. A subset of these formulations with ratios of ingredients by weight is given in Table 3.4. These samples were arbitrarily labelled A to E for the purposes of discussion in this thesis.

\subsubsection{Characterization}

As can be seen in Figure 3.10, transparencies well over $90 \%$ can be achieved with reasonable conductivities of $11 \mathrm{~S} / \mathrm{cm}$ as documented in Table 3.5. Not surprisingly, there is a strong negative correlation between the thickness of the film and its transmission, with the exception of sample D, which has a low transmission relative to its comparatively small thickness. This should not come as a surprise, however, given that 


\begin{tabular}{c|c|c|c|c|c}
\multirow{2}{*}{ Sample } & \multicolumn{2}{|c|}{ Composite ratio by weight } & \multicolumn{4}{|c}{ PEDOT ratio by weight } \\
\cline { 2 - 6 } & Silica sol-gel & PEDOT & Imidazole & CB40 & EDOT \\
\hline A & 1 & 1.5 & 0.5 & 20 & 1 \\
B & 1 & 1.5 & 0 & 20 & 1 \\
C & 1 & 2 & 1 & 20 & 1 \\
D & 1 & 2.5 & 1 & 23 & 1 \\
E & 1 & 1.5 & 1 & 23 & 1
\end{tabular}

Table 3.4: Ratios by weight of sol-gel and PEDOT mixtures for the composite as well as ratios by weight of various constituents of the in situ polymerization mixture for PEDOT.

\begin{tabular}{c|c|c|c|c|c} 
Sample & $\mathrm{R}_{s}[\Omega / \square]$ & $\mathrm{d}[\mathrm{nm}]$ & $\rho[\Omega \mathrm{cm}]$ & $\sigma[\mathrm{S} / \mathrm{cm}]$ & $\begin{array}{c}\text { Thickness } \\
\text { measurement } \\
\text { method }\end{array}$ \\
\hline \hline $\mathrm{A}$ & $27000 \pm 7000$ & 174 & $0.5 \pm 0.10$ & $2.1 \pm 0.5$ & SEM \\
$\mathrm{B}$ & $8700 \pm 500$ & 375 & $0.33 \pm 0.02$ & $3.1 \pm 0.2$ & SEM \\
$\mathrm{C}$ & $90000 \pm 40000$ & 10 & $0.09 \pm 0.04$ & $11 \pm 5$ & Optical profilometer \\
$\mathrm{D}$ & $160 \pm 10$ & 146 & $0.0023 \pm 0.0002$ & $430 \pm 40$ & Optical profilometer \\
$\mathrm{E}$ & $150 \pm 20$ & 1000 & $0.015 \pm 0.002$ & $65 \pm 6$ & Optical profilometer
\end{tabular}

Table 3.5: Conductivities and thicknesses of various PEDOT/silica samples.

sample D has the highest PEDOT content, which would also explain its exceptional conductivity of $430 \mathrm{~S} / \mathrm{cm}$.

The work function of the composite can be calculated from the CV measurements as detailed in Appendix A. As the composition of the samples is slightly different, a variation in the work function is expected. On average the work function appears to fluctuate around $5 \mathrm{eV}$ depending on the composition of the top layer. The PE$\mathrm{DOT} /$ silica work function was calculated to be $\phi_{P E D O T / \text { silica }}=5.02 \pm 0.07 \mathrm{eV}$. 


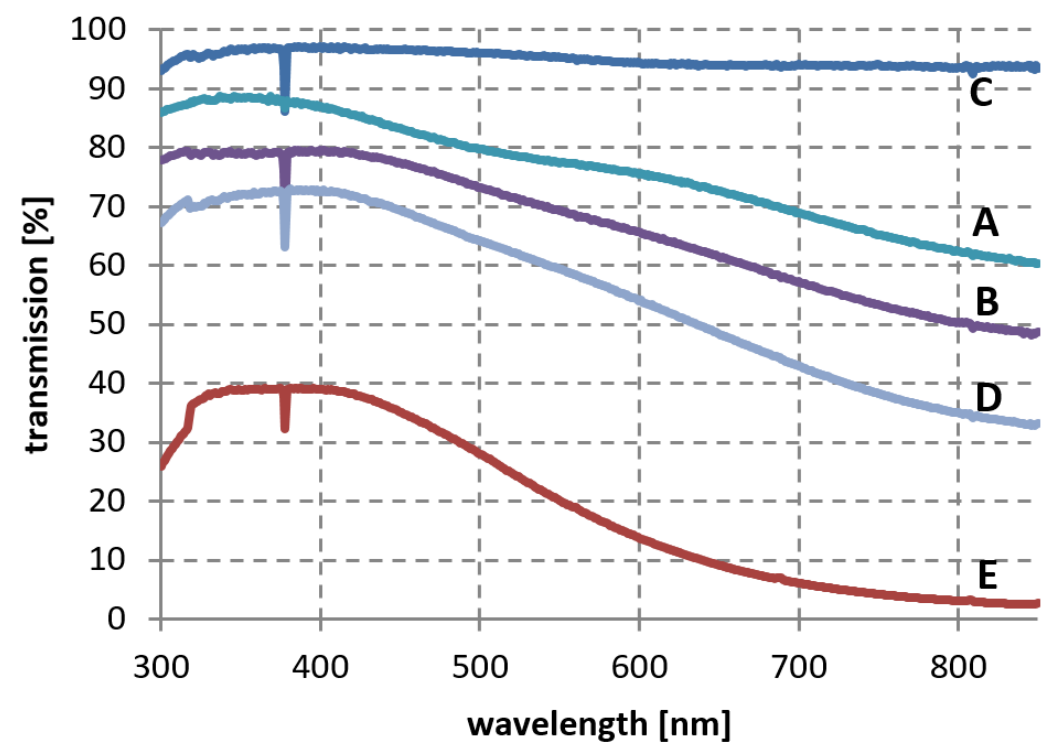

Figure 3.10: Transmission spectrum of PEDOT/silica composites. The irregularities in the spectrum at $380 \mathrm{~nm}, 560 \mathrm{~nm}, 690 \mathrm{~nm}$, and $810 \mathrm{~nm}$ are measurement artifacts.

\subsubsection{Devices}

The PEDOT/silica composite devices were made on 1-2 $\Omega \mathrm{cm} n$-type silicon wafers. The wafers were cleaned using the RCA protocol prior to processing. No intentional oxide was grown on the wafers. Backside aluminum was deposited by e-beam evaporation and sintered in forming gas at $450^{\circ} \mathrm{C}$ for 10 minutes. The PEDOT/silica composite was prepared and deposited as described above. The structure was completed by screen printing a silver ink (Dupont 5028) finger grid.

The linearity of the $1 / \mathrm{C}^{2}$ vs $\mathrm{V}$ plot for these devices indicates a diode behaviour. The extracted work functions are shown in Figure 3.11. All composites appear to have a high enough work function to produce minority carrier devices on $n$-type silicon, making the in situ PEDOT/sol-gel silicate a useful material for this project.

The current-voltage characteristic of sample D shown in Figure 3.12 indicates a substantial series resistance of approximately $150 \Omega$ that cannot be accounted for 


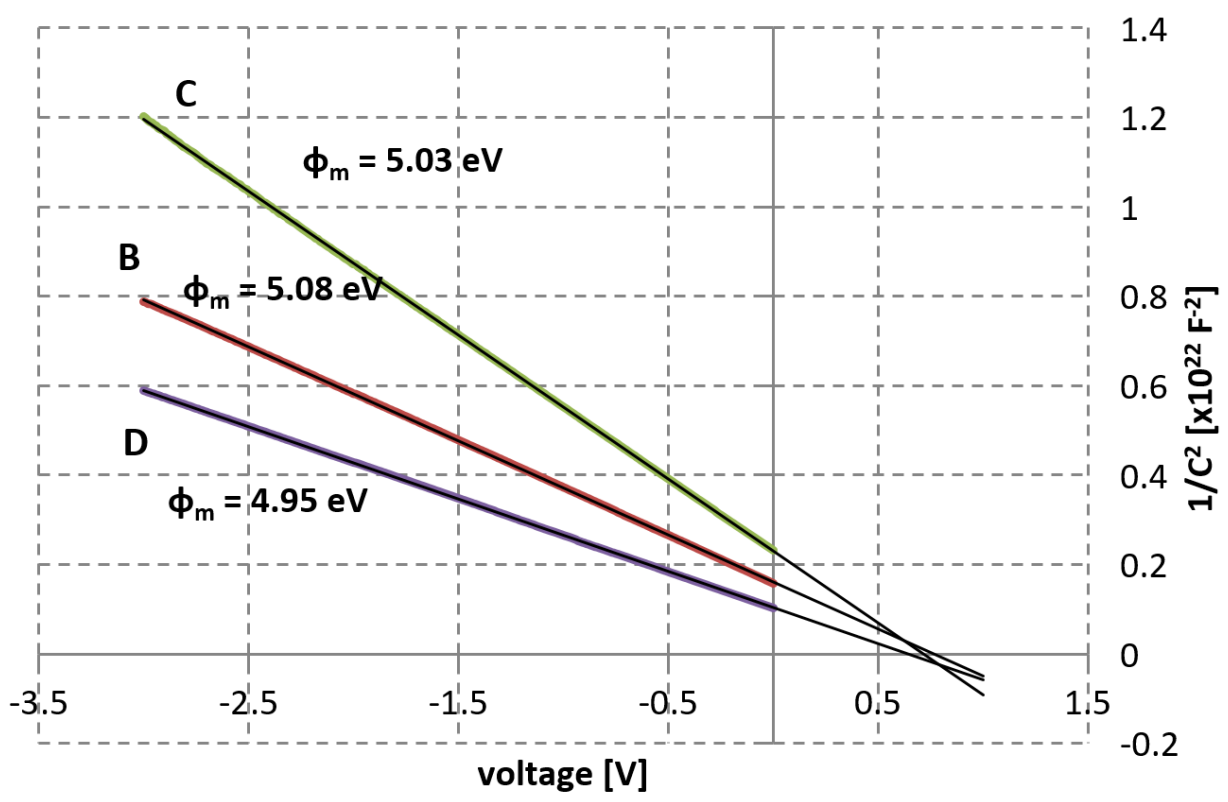

Figure 3.11: CV characteristics of Schottky junctions made with $n$-type silicon (1$2 \Omega \mathrm{cm}$ ) and PEDOT/silica composites of slightly different compositions. The areas of the devices are different. The resistivity of the substrate was taken to be $1.5 \Omega \mathrm{cm}$ for these calculations. This assumption adds an uncertainty of only $0.01 \mathrm{eV}$ to the extracted work function value. 


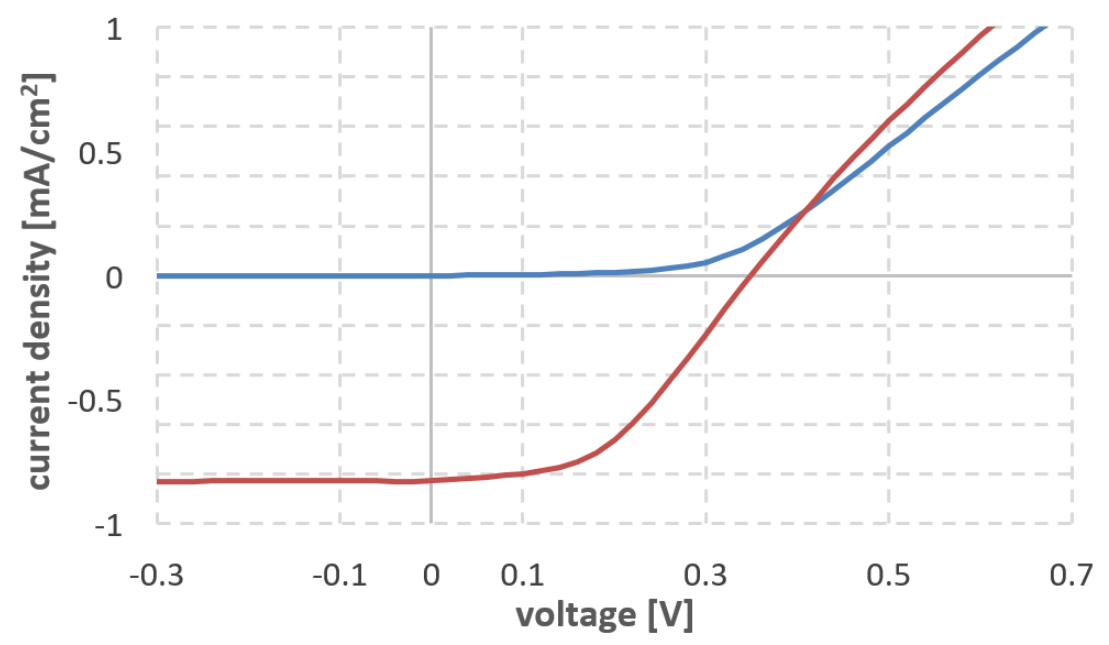

Figure 3.12: Sample D in the dark and under halogen lamp illumination.

by the resistance of the PEDOT/silica composite. This would seem to indicate that there exists an insulating layer between silicon and PEDOT after all. A layer of native oxide could have developed during processing. Alternatively, a layer of silicate at the silicon surface can be acting as a barrier. Accumulation of such a layer is possible as silicate would have a higher affinity for the silicon surface than PEDOT since this is the mechanism that provides a better adhesion to the substrate compared to in situ PEDOT alone.

\subsubsection{Compatibility with Nanostructures}

As can be seen in Figure 3.13, excellent penetration of the composite into a high aspect ratio silicon nanostructure is possible when dehydrating in an evacuated environment. The details of the deposition procedure will be discussed in Chapter 4 .

It is worth noting that just like in the case of in situ PEDOT this structure is prone to the light attenuation problem. Despite having excellent transparency at small thicknesses, at around $1 \mu \mathrm{m}$, the PEDOT/silica composite will block most of the light from reaching the substrate, making the device useless as a solar cell. A 


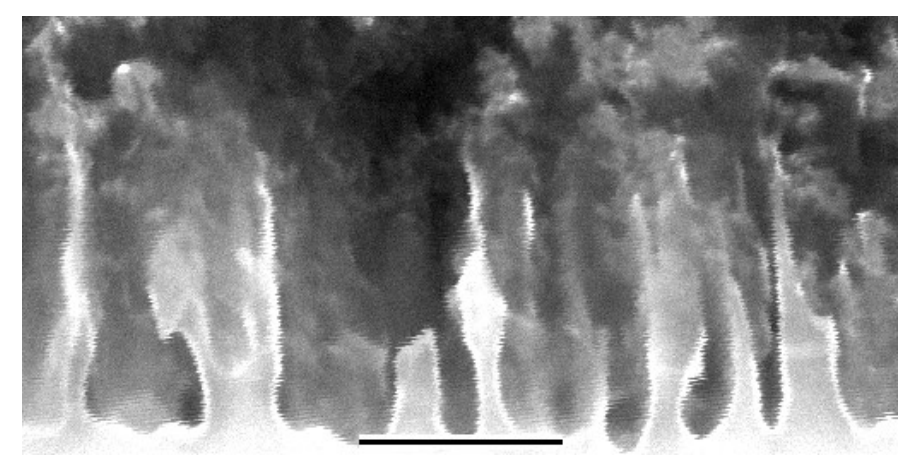

Figure 3.13: SEM image of reactive-ion-etched silicon grass with deposited PE$\mathrm{DOT} /$ silica composite.

careful balance must be reached between the transparency and conductivity of the composite to maximize the solar cell efficiency. As opposed to the in situ PEDOT, however, the composite has the advantage of an extra degree of freedom for finding this balance: the proportion of the silicate to PEDOT can be adjusted to control the optical transmission. Figure 3.14 shows the conductivity and optical loss of the PEDOT/silica per nm of the composite. There is not enough data to make quantitative observations on the effect of diluting in situ PEDOT with the silica sol-gel. However, it can be qualitatively observed that the optical loss seems to decrease with larger silica content. This effect would allow for deposition of thick conductive films with acceptable transmittance on structured surfaces. As one might expect, however, the conductivity decreases with reduced PEDOT content. Chapter 4 will discuss finding an optimal balance of conductivity and transmission in filled nanostructures.

\subsection{Conclusion}

There exist various formulations of the conductive polymer PEDOT that have both excellent conductivity and the right work function for creating the desired minority carrier MIS device on an $n$-type substrate. However, if large aspect ratio surface nanostructuring is to be used to increase light absorption and eliminate the need for 


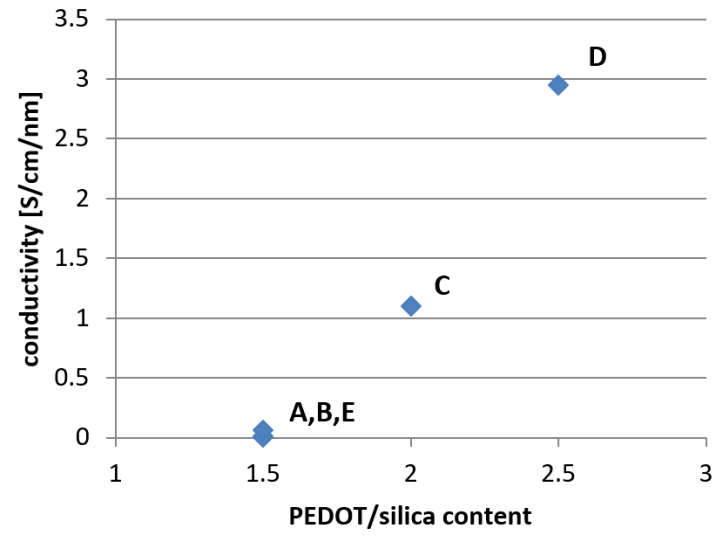

(a)

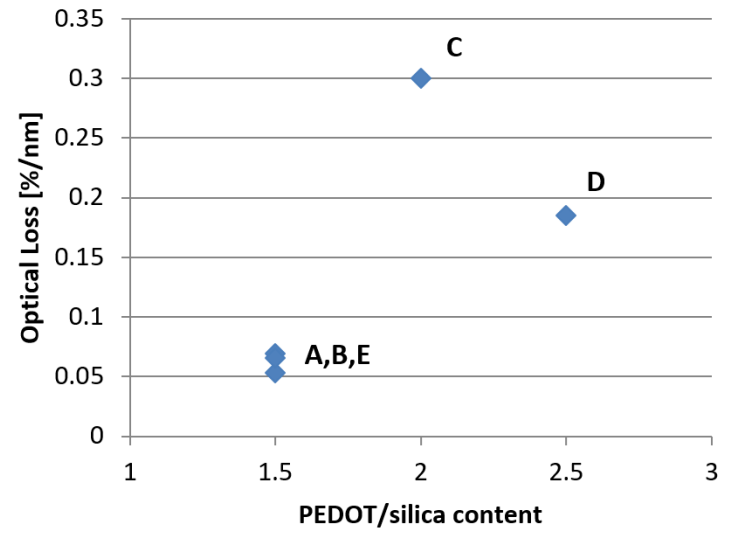

(b)

Figure 3.14: Electrical (a) and optical (b) properties of the PEDOT/silica composites showing increasing conductivity and optical loss with increasing PEDOT content.

costly anti-reflection coatings, a combination of various formulations will be required to maximize solar cell performance. The proposed structure is sketched out in Figure 3.15 .

The PEDOT formulation that penetrates the surface nanostructure should be the PEDOT/silica composite. It would ensure excellent adhesion to the silicon substrate. The transparency/conductivity balance must be adjusted such that the resistive losses due to the low conductivity of the composite balance the optical losses due to the thick conductive layer. The amount of this composite deposited into the structure would need to be carefully controlled to just cover the nanostructure and no more to avoid any further, unnecessary resistive losses. The top PEDOT layer can be as conductive as possible to allow for large spacing of the finger grid electrode. This layer can be made relatively thin to minimize attenuation loss. Both PEDOT:PSS and in situ PEDOT are appropriate for this purpose. Since the composite material will serve as an adhesion layer for the silicon substrate, the poor adhesion to the surface will not be an issue with the in situ PEDOT. As such it might be the most appropriate material choice as its conductivity can be expected to be higher than that of PEDOT:PSS. 


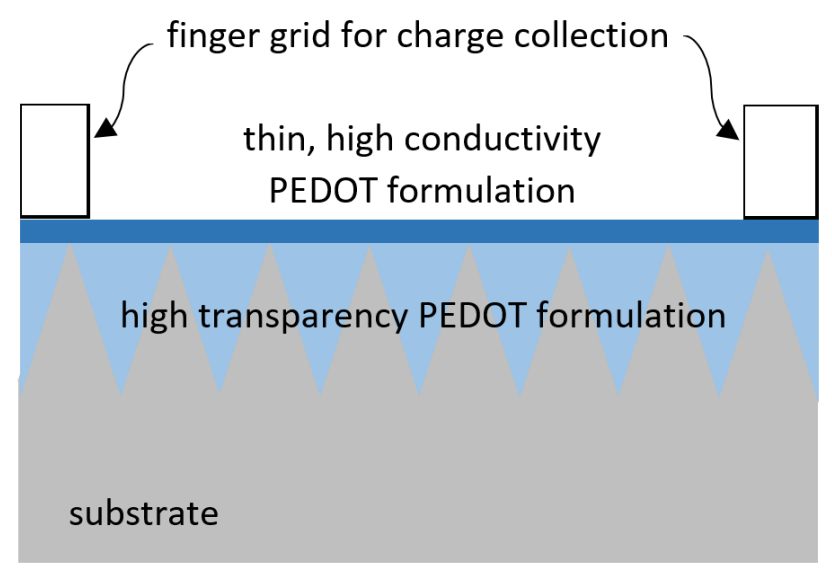

Figure 3.15: Sketch of a nanostructured solar cell that uses two types of PEDOT: one for best optical transparency without compromising the transition of charge up to the top of the nanostructure and another, more conductive, thin layer for horizontal transmission of charge to the finger grid electrodes for collection.

However, if comparable conductivity can obtained from a PEDOT:PSS formulation, the ease of deposition would make such a product more desirable.

Alternatively, a thin conformal layer of PEDOT deposited over the nanostructure as shown in Figure 3.16 would solve the light attenuation problem as well. For such a scheme to be effective, however, the conductivity of this PEDOT must be high to reduce the spacing of the charge collecting finger grid. The merits of both the twophase filling and conformal depositions schemes will be further assessed in Chapter 4 . 


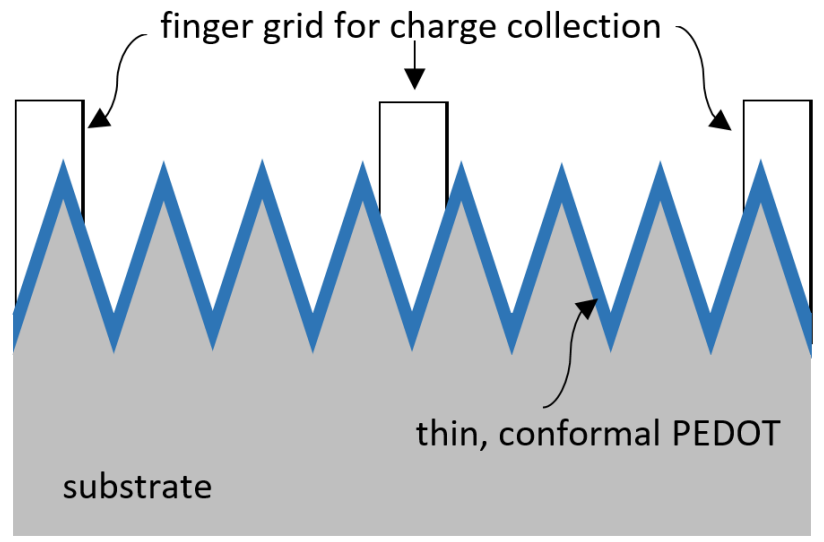

Figure 3.16: Sketch of a nanostructured solar cell with a highly conductive conformal layer of PEDOT. The spacing of the finger grid is limited by the conductivity of PEDOT. 


\section{Chapter 4}

\section{Nanostructuring}

Silicon is quite efficient at absorbing the blue wavelengths of the solar spectrum, however, most of the red spectrum passes through a relatively thin Si device unabsorbed as illustrated by the absorption coefficient in Figure 4.1. Additionally, up to $70 \%$ of the light in the blue end of the spectrum is lost to reflection before it has a chance to be absorbed by the substrate and over $30 \%$ is lost in the same manner elsewhere in the spectrum. To make better use of the available energy, solar cells have traditionally been capped with an anti-reflecting film [9]. However, a different approach is possible. The surface of Si can be roughened to create multiple reflections back into the substrate effectively "trapping" the light. By increasing the pathlength of the light at the surface of the cell, the light capture is improved.

The most common, and a rather elegant way of nanostucturing a Si surface is an anisotropic wet chemical etch. It is an economical and commercially viable technique of creating a pyramidal structure on the Si surface seen in Figure 4.2 [6,9]. Such structuring reduces the reflectivity of the silicon surface by about a factor of two. ${ }^{1}$ However, a greater improvement in reflectivity is possible.

Most of the efficiency gain in a nanostructured device is expected to come from

\footnotetext{
${ }^{1}$ In practice, such pyramidal surface structures are combined with antireflection coatings for a greater improvement in surface reflectivity [9].
} 


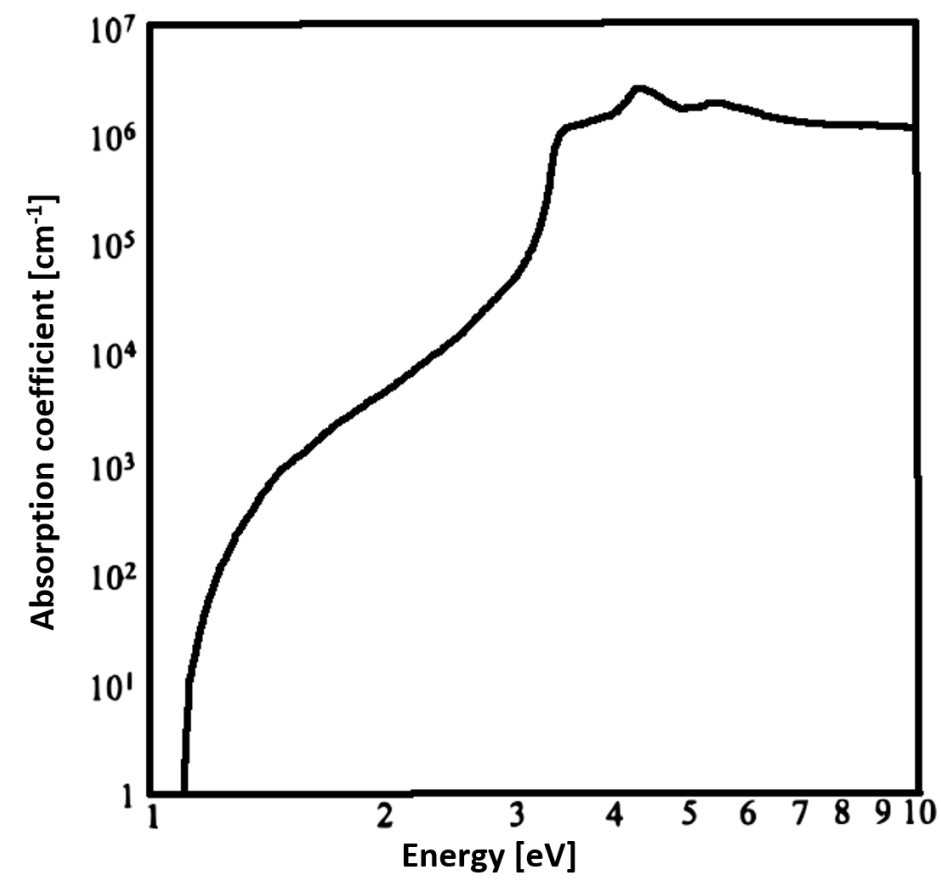

Figure 4.1: Absorption coefficient of silicon. The light at lower wavelengths (higher energies) is absorbed more readily then at higher wavelengths (lower energies). After [8]. 


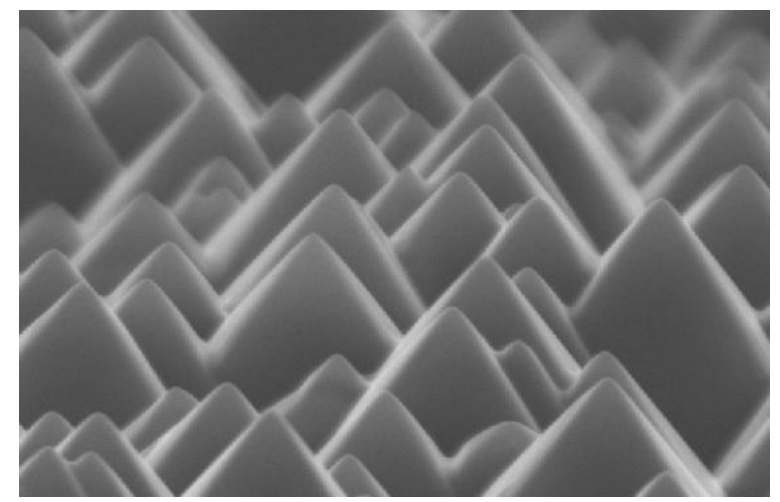

(a)

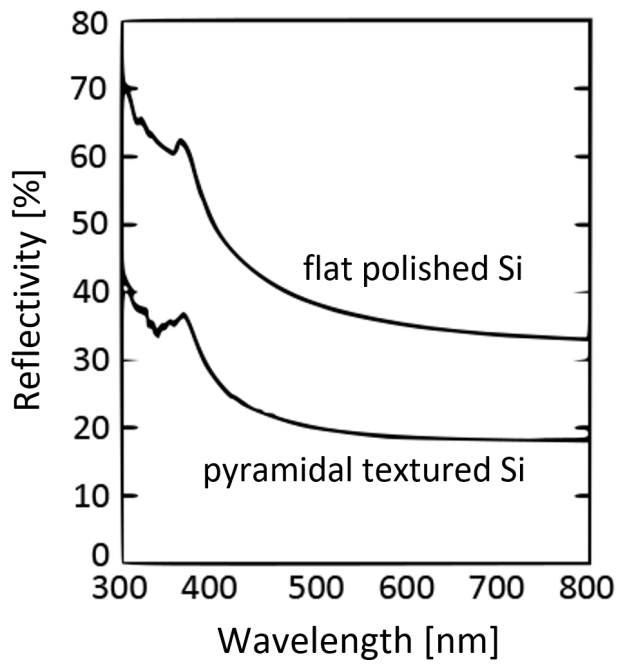

(b)

Figure 4.2: (a) SEM image of pyramidal structure in silicon created with an anisotropic alkaline etch. After [9]. (b) Reflectivity of such a structure with the reflectivity of polished silicon surface shown for reference. After [115].

a reduction in reflectivity as simulated with effective medium theory in Section 4.1 and observed experimentally and in simulation by many research groups [111-114]. This should lead to as much as a three-fold increase in the short circuit current density of the PV device. A further improvement in the current density can be expected from increased absorption due to scattering. From the comparison of single junction and multi-junction PV device performance reported in the literature [15], this improvement can be roughly estimated to be up to one-and-a-half times. Practically, however, the increased surface recombination due to the surface area increase can be expected to reduce the fill factor. Additionally, the light attenuation in the PEDOT layer would reduce the short circuit current. Both of these setbacks are explored in further detail in Section 4.4 . 


\subsection{Structure Requirements}

According to the effective medium theory, the reflection is minimised when the refractive index is varied linearly and gradually from that of one medium to another. In practice, this effect can be achieved by structuring the surface of one of the media such that in the interpenetrating structure the volume fraction of this medium

changes smoothly from $0 \%$ to $100 \%$. However, such behaviour can only be achieved if the periodicity/feature size of the structure is smaller than the wavelength of interest. Since the entire optical spectrum is relevant, this would set the upper limit of the structure feature size at about $300 \mathrm{~nm}$.

On the upper end of the spectrum of interest the low reflectivity cut-off of such a structure is a function of the structure height. A simulation of the reflectivity shows that structures in excess of $1 \mu \mathrm{m}$ tall are required to effectively trap light in the infra-red region of the spectrum as shown in Figure 4.3. The reflectivity is calculated by breaking the nanostructure up into horizontal slices, each with its own volume fraction of Si. Thin film reflectivity calculations are then performed on the stack recursively $[32,34,35,116]$. The full code used to calculate the expected reflectance can be found in Appendix B.

For the most effective anti-reflection surface, high aspect ratio structures with graded volume fraction of substrate material are required with column diameters smaller than $300 \mathrm{~nm}$ and heights in excess of $1 \mu \mathrm{m}$. When optimized such surface structuring might even be able to eliminate the need for costly antireflection coatings.

\subsection{Nanostructuring Techniques}

There are many schemes for producing nanostructures on substrate surfaces. Some involve growth of structures [117], others are deposited [118], and some are etched 


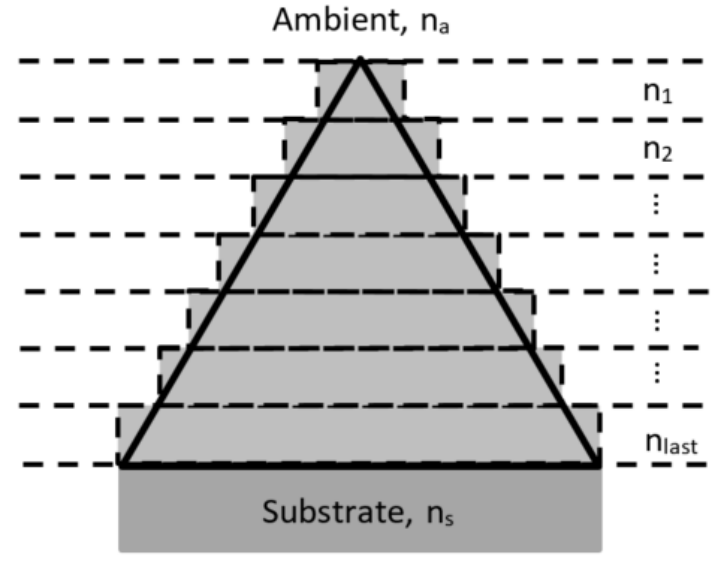

(a)

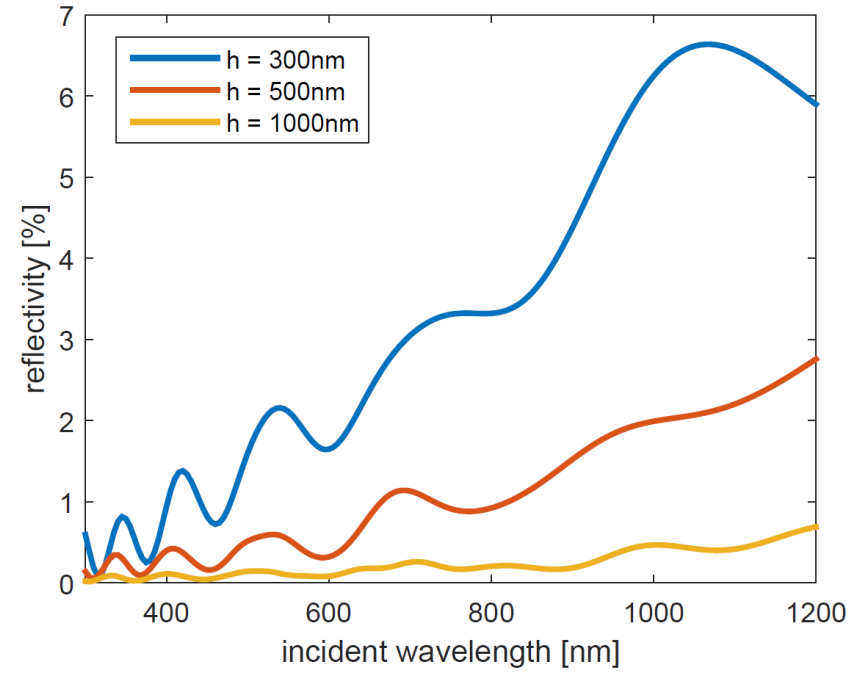

(b)

Figure 4.3: Simulated reflectivity of a nanostructured surface in air with various feature sizes and heights (a) approximation to the nanostructure geometry used in the numerical calculations (b) resultant reflectivities.

$[111,112,119-123]$. In this work, the so-called "silicon grass" produced in a selfmasking dry etch and metal-assisted wet etched structures were used.

\subsubsection{RIE silicon grass}

Silicon grass is usually an unwanted feature of a high aspect ratio dry reactive ion etch (RIE) of silicon. This feature appears when the partial pressures of $\mathrm{SF}_{6} / \mathrm{O}_{2}$ were not chosen properly. However, this technique has been long since adapted for producing so called "black silicon", useful as an anti-reflection treatment in solar cells [119-122]. The process for silicon grass formation is illustrated in Figure 4.4. Any stray dust particles and native oxide act as a mask while the fluorine radicals $\left(\mathrm{F}^{*}\right)$ preferentially remove silicon. The oxygen radicals $\left(\mathrm{O}^{*}\right)$ oxidize the tops and the sidewalls of the nanostructure, while the physical bombardment with ions from the plasma etches it away along with any stray dust and debris. The partial pressures 


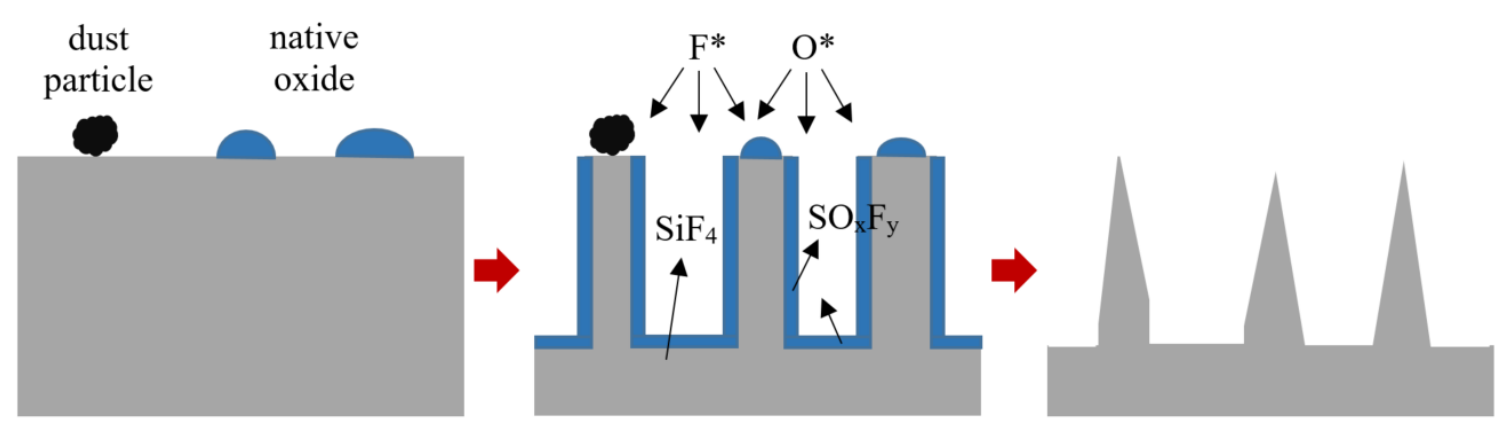

Figure 4.4: Formation of silicon grass by reactive ion etching in $\mathrm{SF}_{6} / \mathrm{O}_{2}$ environment.

of $\mathrm{SF}_{6}$ and $\mathrm{O}_{2}$ can be chosen such that vertical etch of silicon dominates while the oxidising portion of this environment maintains a random mask of $\mathrm{SiO}_{2}$ islands at the surface thus forcing the formation of thin silicon pillars. A damage removal treatment post-RIE etch would help minimize surface recombination in devices constructed on such substrates. A short mixed acid etch can be used for this purpose [119].

The trouble with this nanostructuring technique is that the etch parameters necessary to achieve this effect are specific for each and every RIE machine and must be determined experimentally [120]. A reliable recipe for high quality black Si found experimentally for the PlasmaTherm SLR-772 ECR etcher in the Carleton University Fabrication Laboratory is given in Appendix C. The resultant structure and its reflection spectrum are shown in Figure 4.5.

\subsubsection{Gold-catalized wet etch}

A wet chemical etch is usually preferable in commercial manufacturing as a more economical and scalable option. Howard Branz developed an etch that uses $\mathrm{HF}: \mathrm{H}_{2} \mathrm{O}_{2}: \mathrm{H}_{2} \mathrm{O}$ chemistry with colloidal gold particles [123]. Whereas Branz added the colloidal gold solution to the etchant and sonicated the substrate in this solution, the current research found that spin-casting and drying the particles on the surface prior to the etch yielded surfaces with much lower reflection. The etch procedure and 


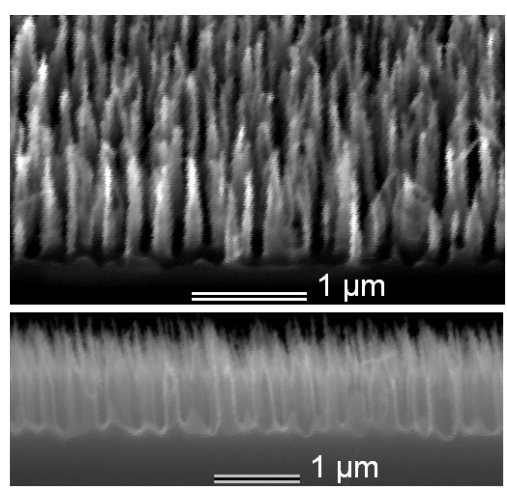

(a)

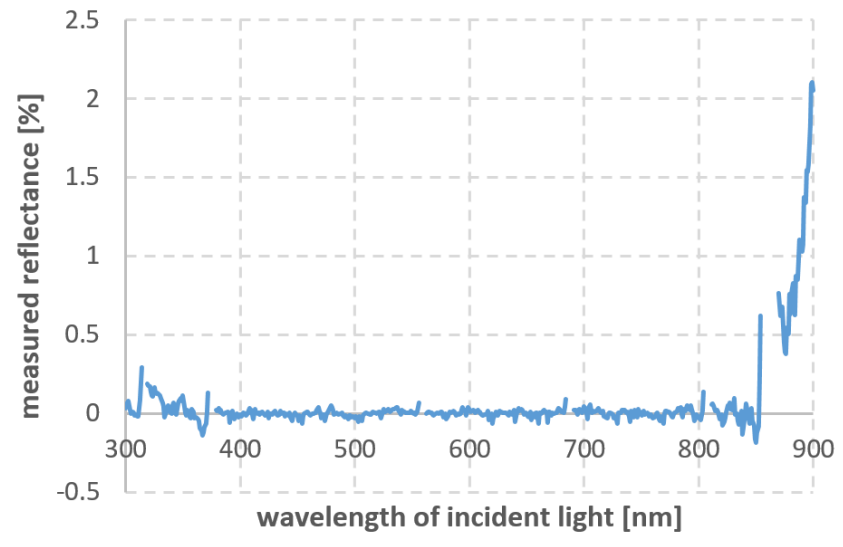

(b)

Figure 4.5: RIE silicon grass nanostructuring and corresponding reflection spectrum; (a) SEM image of RIE silicon grass (b) reflectivity of silicon grass at a single angle of $20^{\circ}$ measured with a Lamda900 spectrometer.

the SEM image of the resultant surface are shown in Figure 4.6. The Au particles catalyse the preferential oxidation of the Si surface in the particles' vicinity and the HF etches the resultant oxide continuously. Thus, the gold particles "burrow" into the silicon substrate creating a structured surface. The depth of the structure is determined by the duration of the etch. The gold can subsequently be removed with aqua regia. If the size and the positioning of the $\mathrm{Au}$ nanoparticles can be precisely controlled, so can the morphology of the structured surface.

\subsection{Atomic Layer Deposition}

Thermal growth of a dielectric is not the only method of producing an interfacial layer for an MIS device. Atomic layer deposition (ALD) has been of increasing interest in the scientific community in recent years. This deposition technique provides very conformal and continuous films with excellent thickness control on the molecular scale. Additionally, a variety of dielectrics deposited in this manner can be explored for constructing MIS diodes. As such, insulators with a lower potential barrier than 

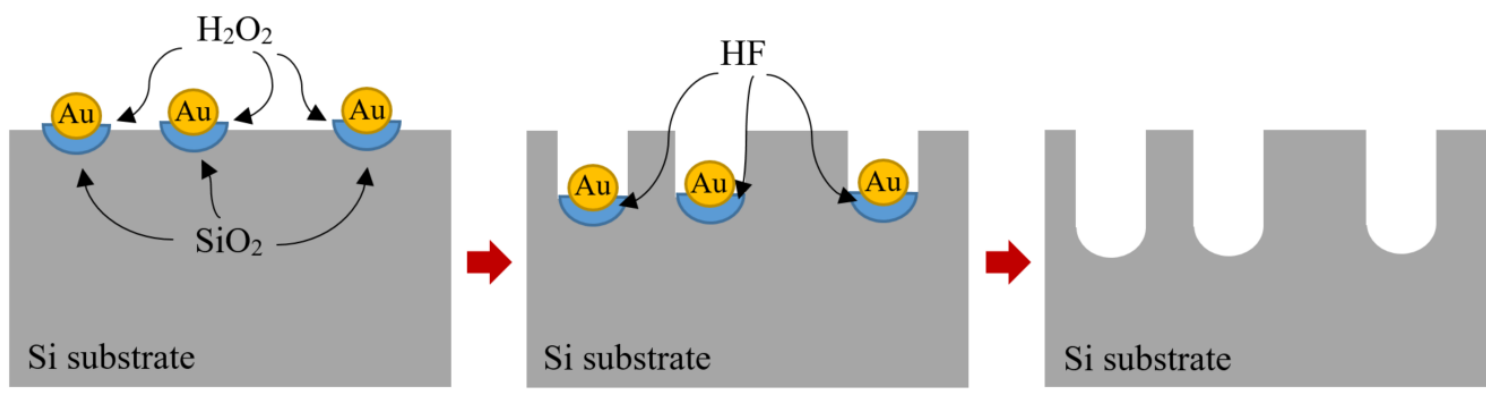

(a)

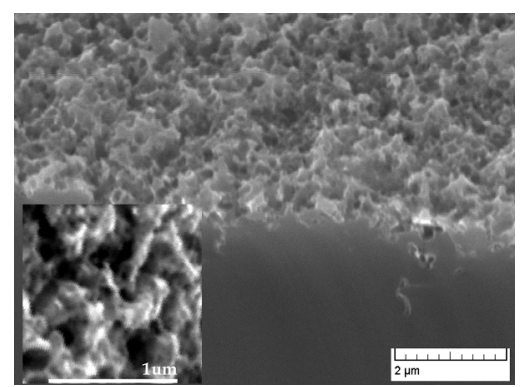

(b)

Figure 4.6: Gold-assisted chemical wet etch of silicon (a) process illustration (b) SEM image of the result.

$\mathrm{SiO}_{2}$ can be used to reduce the sensitivity of the diode to the thickness of the dielectric, increasing the tolerances in the fabrication protocols.

The general overview of an ALD process was given in Chapter 2, but the illustration of the process is reproduced here for reference (Figure 4.7). The $\mathrm{Al}_{2} \mathrm{O}_{3}$ was $\mathrm{ALD}$ grown on the Si surface using $\left[\mathrm{MeC}\left(\mathrm{N}^{i} \mathrm{Pr}\right)_{2}\right] \mathrm{AlEt}_{2}$ as the $\mathrm{Al}$ precursor and water as the oxygen precursor with $\mathrm{N}_{2}$ as the purging gas. The maximum growth rate for this process was reported as $2.7 \AA /$ cycle [57]. Prior to ALD, the substrates were exposed to air at $250^{\circ} \mathrm{C}$ for six hours to saturate the surface with hydroxyl groups for effective binding of the precursor. 
1. $1^{\text {st }}$ precursor

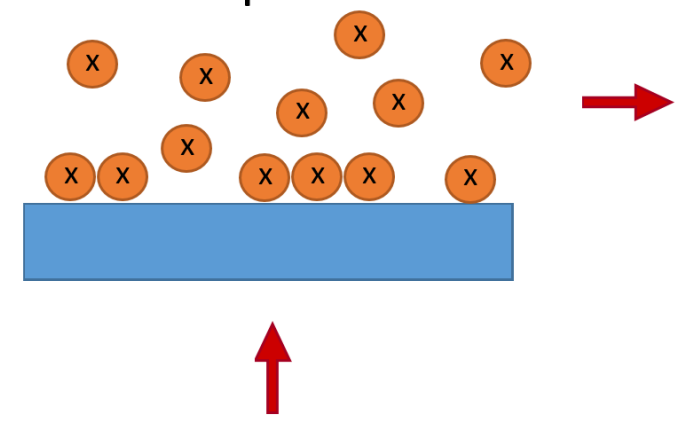

4. Purge

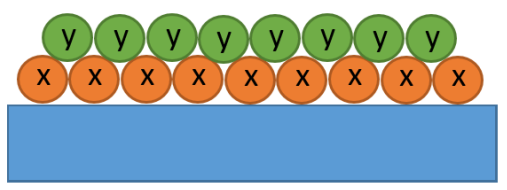

\section{Purge}

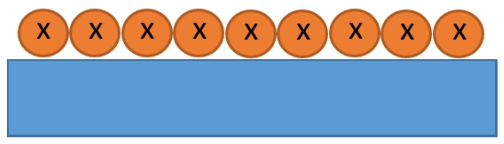

$\downarrow$

3. $2^{\text {nd }}$ precursor

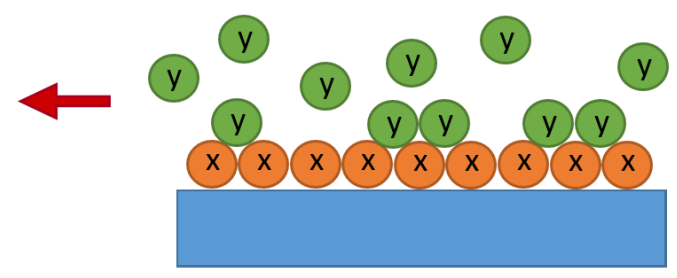

Figure 4.7: Illustration of the ALD process. First precursor is adsorbed to the surface until a complete monolayer is formed, then evacuated with a purging gas. The second precursor reacts with the new surface until a complete monolayer of the desired material in produced, $\mathrm{Al}_{2} \mathrm{O}_{3}$ in this case. The rest of the precursor is, again, purged. The cycle is repeated until the desired thickness is reached. 


\subsection{Problems and Solutions}

Several problems are anticipated in relation to nanostructured substrates. These issues pertain to surface states, PEDOT filling, and PEDOT shadowing.

The surface structuring increases the effective surface area of the device. While this should allow for more efficient charge extraction, it can significantly increase the effective surface trap density, which leads to loss of current. Assuming a structuring geometry optimal for minimizing reflection, i.e. a forest of cones and assuming moderate dimensions of $\mathrm{h}_{o}=1000 \mathrm{~nm}$ and $\mathrm{r}_{o}=300 \mathrm{~nm}$ (Figure 4.8), the surface area increases by approximately a factor of 5.5. This would increase the recombination current from approximately $1.8 \mathrm{~mA} / \mathrm{cm}^{2}$ to $10 \mathrm{~mA} / \mathrm{cm}^{2}$. Appendix D provides details of this estimation. ${ }^{2}$ With a maximum current density of $33 \mathrm{~mA} / \mathrm{cm}^{2}$ for a silicon based device, this increase in surface recombination can result in a solar cell efficiency drop by a factor of 3 or more. As such, efficient surface passivation becomes that much more important in structured devices.

It was discovered that when spin-casting protocols for flat substrates are applied to high aspect ratio structures, the PEDOT:PSS tends to sit on the surface of the structure, without filling it as shown in Figure 4.9(a). This contact geometry will considerably compromise charge extraction efficiency, not to mention limiting the MIS diode formation to the tips of the nanostructures. Ideally, a centrifuge-type deposition device would be most effective at filling the structure while eliminating excess PEDOT:PSS. In this absence of such apparatus, the PEDOT:PSS can be vacuum deposited, as was done in this work. The polymer was drawn into the structure under vacuum, which evacuated air from the structure cavities allowing the polymer to fill them, as well as dehydrating the polymer solution, eliminating the need for a

\footnotetext{
${ }^{2}$ The inversion layer at the surface should reduce surface recombination somewhat, as was discussed in Chapter 2. However, since the Shockley-Read-Hall recombination theory cannot account for this effect, it was not included in this recombination current estimation.
} 


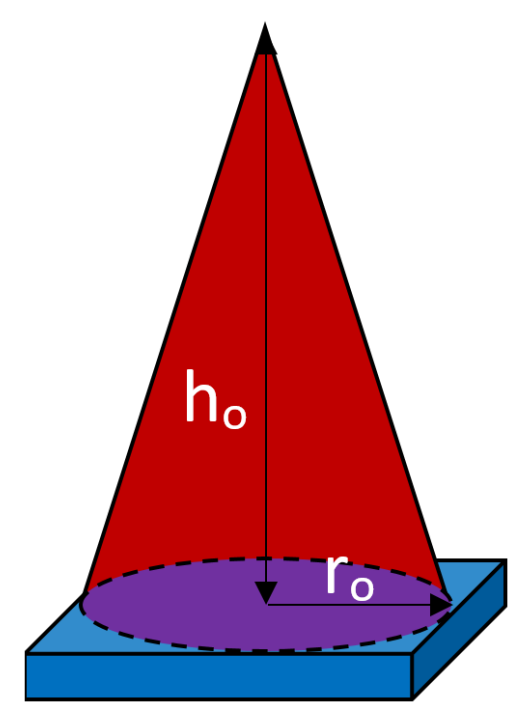

Figure 4.8: Effective surface area increase due to nanostructuring for reflection minimization.

post-deposition bake. The resultant coverage is shown in Figure 4.9(b).

PEDOT in its various formulations has very good transparency properties, as discussed in Chapter 3. However, as with all transparent conductors, there is a tradeoff between transparency and thickness/conductivity. At over $1 \mu \mathrm{m}$ thick, which is necessary to fill ideal surface structures, very little light would reach the silicon substrate, negating any benefit of the surface structure. This PEDOT shading issue can either be resolved with a two-stage filling of Si nanostructures (Figure 4.10(a)) or with a thin conformal layer of PEDOT (Figure 4.10(b)).

Two-stage filling of the nanostructure involves two formulations of PEDOT: one in the structure that maximizes transparency, and one on the surface of the structure that maximizes conductivity. To minimise the power loss in the highly transparent PEDOT, the ratio of PEDOT to silica in the composite should be chosen such that the losses from the optical attenuation and resistivity are balanced. The conductivity and attenuation coefficient of a PEDOT/silica composite were analysed using percolation theory. Based on Lee's data [124], dependence of conductivity on PEDOT/silica ratio is consistent with a three-dimensional matrix near percolation threshold. The 


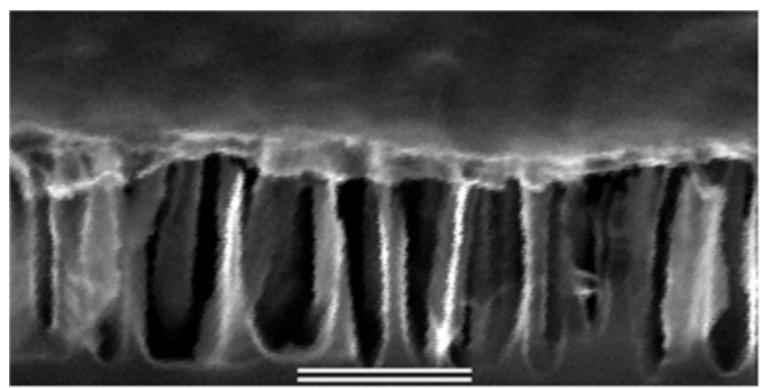

(a)

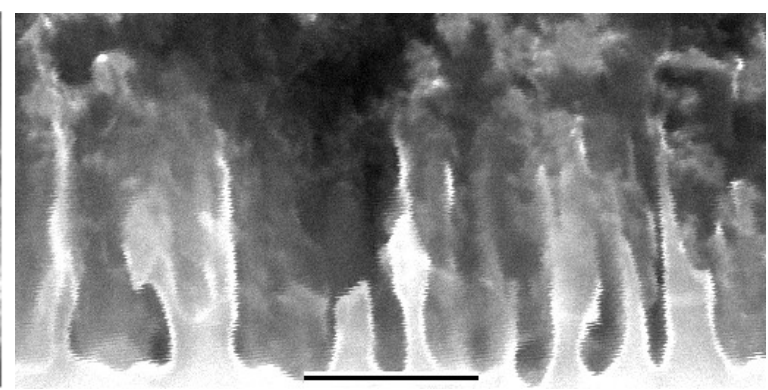

(b)

Figure 4.9: Filling of RIE Si nanostructure with a PEDOT/silica composite. (a) Composite was spin cast and dried on the hotplate in air. (b) Composite was deposited on the substrate and dried in vacuum. The scale bar is $1 \mu \mathrm{m}$.

dependence of the attenuation coefficient of the composite on the PEDOT/silica ratio follows the percolation theory for a two-dimensional matrix below percolation threshold. Using these dependencies the power loss due to filling a $1 \mu \mathrm{m}$ deep nanostructure with a PEDOT/silica composite can be minimized to $0.0005 \%$ using a PEDOT to silica ratio of 0.14 . The procedure for minimizing power loss in a filled nanostructured solar cell is detailed in Appendix E. However, additional reflective power loss of approximately 3\% will result from filling the nanostructure as shown in Appendix F.

A conformal PEDOT coating can be achieved with vapour phase polymerization [125] or ALD. However, the increase of the current path length due to the surface structure would require a smaller finger grid spacing for power loss minimization. A comparative study of minimum power loss for the planarized and structured devices is detailed in Appendix F. The analysis shows that the planarized geometry outperforms the conformal coating on the structured surface with a minimum power loss of approximately $13 \%$ with $26 \mathrm{~nm}$ thick PEDOT and $0.1 \mathrm{~cm}$ finger grid spacing as compared to a minimum loss of $20 \%$ (with PEDOT thickness of $41 \mathrm{~nm}$ and grid spacing of $0.06 \mathrm{~cm}$ ). Even when the additional reflective losses due to filling the nanostructure are taken into account, the planarized geometry is still expected to outperform the 


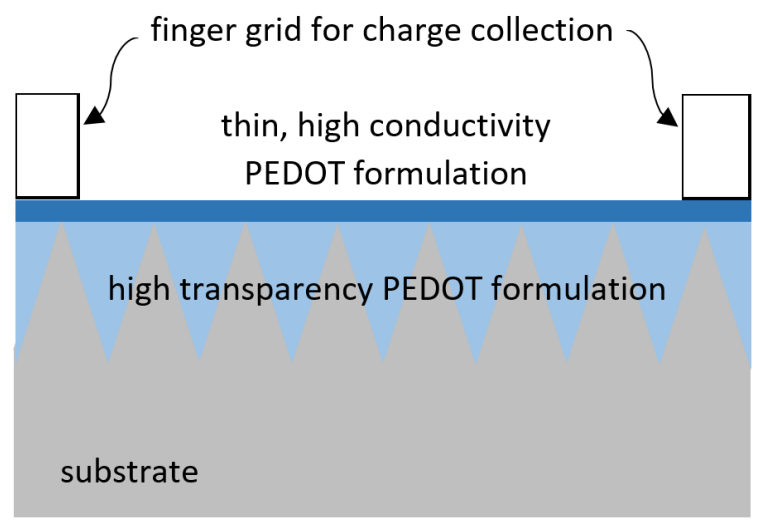

(a)

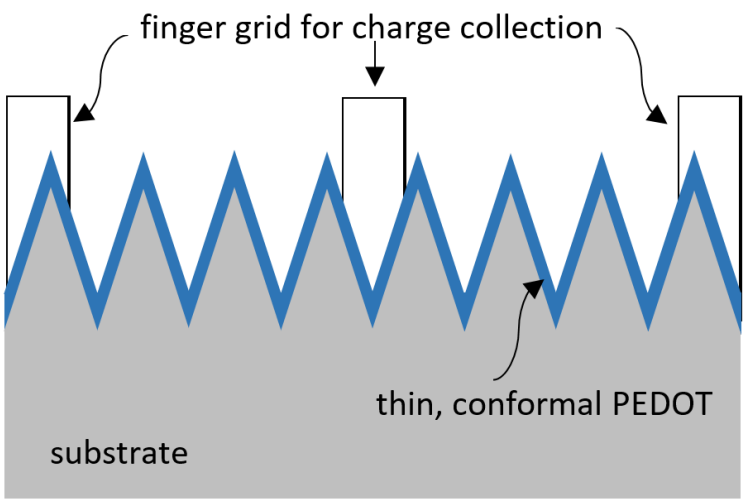

(b)

Figure 4.10: PEDOT deposition schemes for solving the PEDOT shading problem (a) two types of PEDOT: one for best optical transparency without compromising the transition of charge up to the top of the nanostructure and another, more conductive, thin layer for horizontal transmission of charge to the finger grid electrodes for collection. (b) thin highly conductive conformal layer of PEDOT; the spacing of the finger grid is limited by the conductivity of PEDOT.

conformally coated nanostructure with the reflectivity of the empty structure being below $0.5 \%$, and the filled structure having a reflectivity of about $3 \%$.

\subsection{Devices}

\subsubsection{Fabrication}

To assess the benefits of nanostructuring the substrate, devices on flat and wet-etched substrates were fabricated in parallel. The insulator used was ALD grown aluminum oxide $\left(\mathrm{Al}_{2} \mathrm{O}_{3}\right)$. The diodes were finished with in situ PEDOT. Diodes on RIE silicon grass substrates were also made. Once the substrates were prepared, the rest of the processing followed the same protocol as the wet-etched devices.

To prepare the substrate for the wet-etch, the Si surface was ozone-treated to improve wetting. Colloidal gold particles $(5 \mathrm{~nm})$ were dried on the Si surface in a vacuum oven at room temperature to minimize aggregation. The substrates were 
then etched in an ultrasonic bath of $\mathrm{HF}: \mathrm{H}_{2} \mathrm{O}_{2}: \mathrm{H}_{2} \mathrm{O}$ (1:5:2) for 2 minutes followed by an aqua regia wash to remove the gold. This treatment results in feature size of approximately $300 \mathrm{~nm}$ with an aspect ratio of $1: 1 .^{3}$.

The self-templated dry reactive ion etch was done in PlasmaTherm SLR-772 ECR etcher using a two gas system $\left(\mathrm{SF}_{6}\right.$ and $\left.\mathrm{O}_{2}\right)$. The exact recipe used is detailed in Appendix C. This results in 1:10 aspect ratio black silicon with roughly $100 \mathrm{~nm}$ features.

For all wafers, aluminum is then deposited on the back of the Si substrates and sintered at $400^{\circ} \mathrm{C}$ in $\mathrm{H}_{2}$ briefly to create an ohmic back contact. The back of the sample is then protected with kapton tape to protect the $\mathrm{Al}$ in the following processing.

The substrates are annealed in air at $250^{\circ} \mathrm{C}$ for 6 hours to create hydroxyl groups on the Si surface to nucleate the ALD growth of alumina, which is then deposited. Energy-dispersive X-Ray Spectroscopy (EDS) of the substrate post ALD shows the presence of oxygen and $\mathrm{Al}$ on the surface indicating that an alumina layer has been grown.

To deposit PEDOT in situ on top of the structure, the substrate is dip coated in a mixture of isopropanol, EDOT monomer, Fe(III) tosylate in butanol and imidazole (200:1:20:0.5), dried and then rinsed in deionized (DI) water. It was found that the addition of imidazole considerably increases the conductivity of the film from $36 \mathrm{~S} / \mathrm{cm}$ to over $100 \mathrm{~S} / \mathrm{cm}$ and improves transparency from $60 \%$ to $80 \%$.

\subsubsection{Analysis}

As can be seen in Figure 4.11, the devices show photoresponse, but poor conversion efficiency. The low efficiency may be explained by a combination of thickness of the tunneling insulator layer, surface recombination, and PEDOT shading.

\footnotetext{
${ }^{3}$ Atomic force microscopy (AFM) imaging of the dried gold particles on the Si surface prior to etching reveals aggregation of gold particles into structures of up to $400 \mathrm{~nm}$ in diameter. This explains the large etched feature size compared to the size of colloidal particles
} 
The known inferior passivation properties of deposited films vs grown films could result in surface recombination and contribute to the reduction of the short circuit current [40]. However, in this case, current suppression due to non-optimal thickness of oxide is a more likely explanation. Since the substrate is exposed to air at $250^{\circ} \mathrm{C}$ for 6 hours prior to ALD, a layer of $\mathrm{SiO}_{2}$ likely resulted. The low temperature oxide growth is not well understood, but the measured growth of native oxide on $n$-type substrate of a similar doping density at room temperature suggests that approximately $3 \mathrm{~nm}$ of native oxide will grow in 6 hours [44]. At an elevated temperature of $250^{\circ} \mathrm{C}$ this number can be expected to be higher. On top of this layer, ALD alumina was deposited. Thus, the thickness of the dielectric would suppress the tunneling current and reduce short circuit current of the solar cell. PEDOT shading, of course, would contribute to both low short circuit current and low open circuit voltage, by limiting the amount of light that reaches the substrate as discussed in Appendix E.

The observed low fill factors of $\mathrm{FF} \leq 0.25$ are also consistent with this analysis. This effect is generally attributed to poor extraction of charge from the device. This effect can be due to surface recombination or current-limiting interfacial layers $[126$, $127]$.

Figure 4.12 gives the efficiencies of measured solar cells on RIE substrates. The largest source of error in these measurements was the estimation of the incoming optical power. The devices were measured under a halogen tungsten light, which was approximately aligned for maximum solar cell illumination while attempting to keep the distance between the lamp and the devices constant from measurement to measurement. The incident power was estimated as an average of several measurements taken with a Coherent LaserCheck power meter set to a wavelength of $550 \mathrm{~nm}$. As can be seen in Figure 4.12(b) the device efficiency drops approximately exponentially with the insulator thickness as one might expect, given the exponential dependence of tunneling current on barrier thickness. 


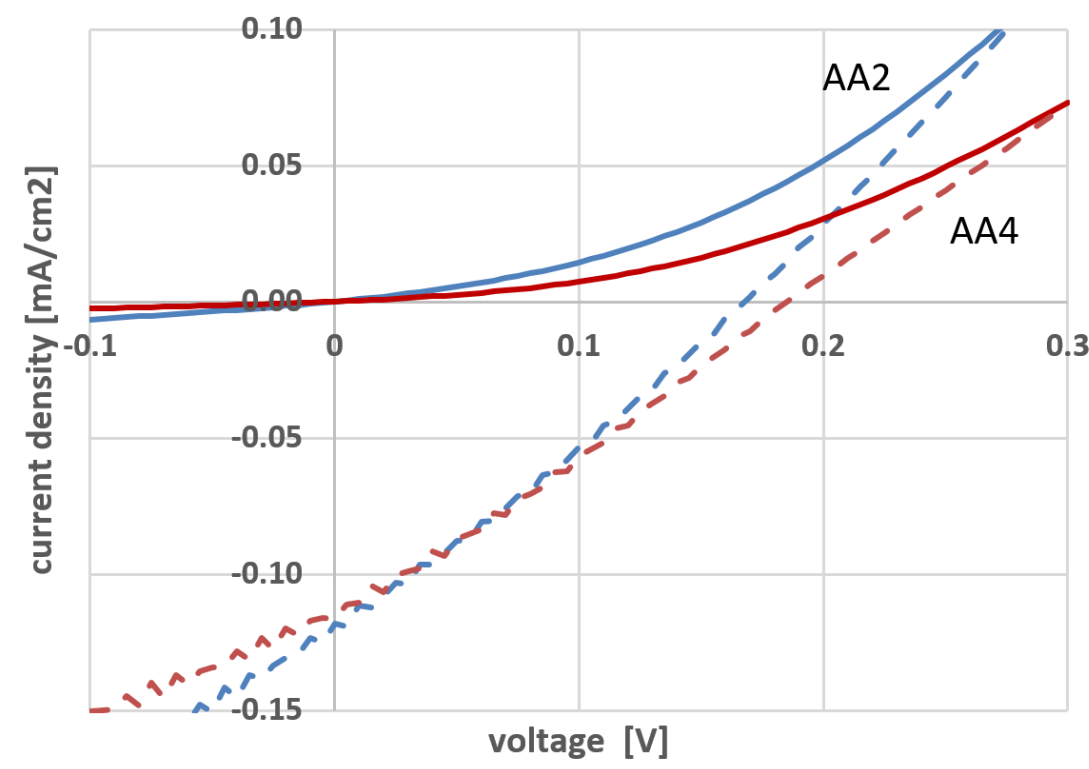

Figure 4.11: Current-voltage characteristics of the two best performing solar cells constructed with $\mathrm{Al}_{2} \mathrm{O}_{3}$ insulator on RIE silicon substrate in the dark and under halogen lamp illumination. The recorded efficiencies are $1.6 \% \pm 0.4 \%$ and $1.7 \%$ $\pm 0.4 \%$.

Despite the non-optimal construction of the cells, they allow for a relative comparison of flat and structured devices. Figure 4.13 shows the efficiency of a set of solar cells on a flat silicon substrate alongside a set of devices on a gold-assisted wet-etched substrate. Comparing the two types of devices, a 5 fold improvement in efficiency of the structured devices vs flat ones is noted [71].

\subsection{Conclusion}

Nanostructuring the substrate appears to be a viable method for increasing solar cell efficiency despite several drawbacks associated with the added complexity. The predicted reduction in efficiency by a factor of three due to the increase in the surface states via the surface area increase seems to be more than compensated, resulting in an observed five-fold increase in efficiency, presumably due to better light capturing 


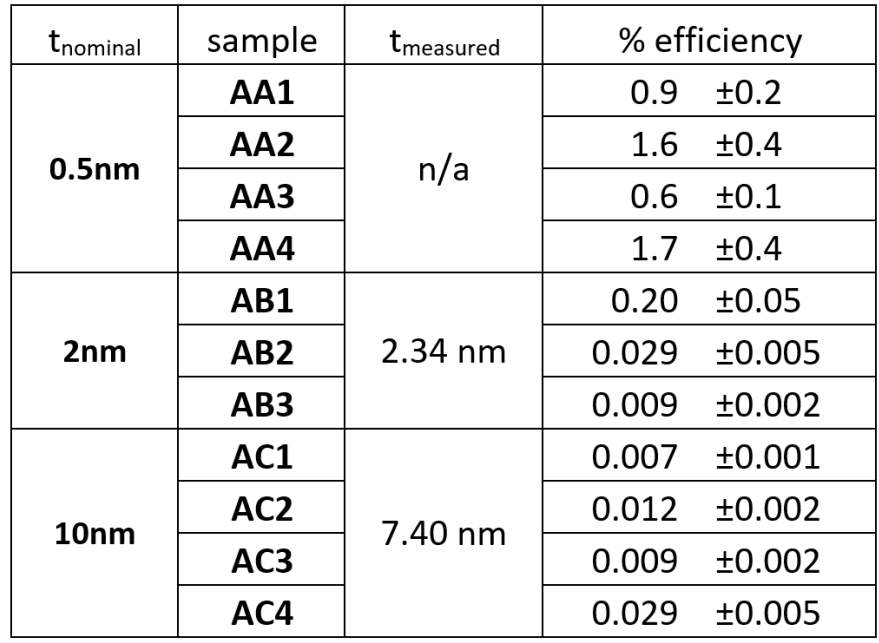

(a)

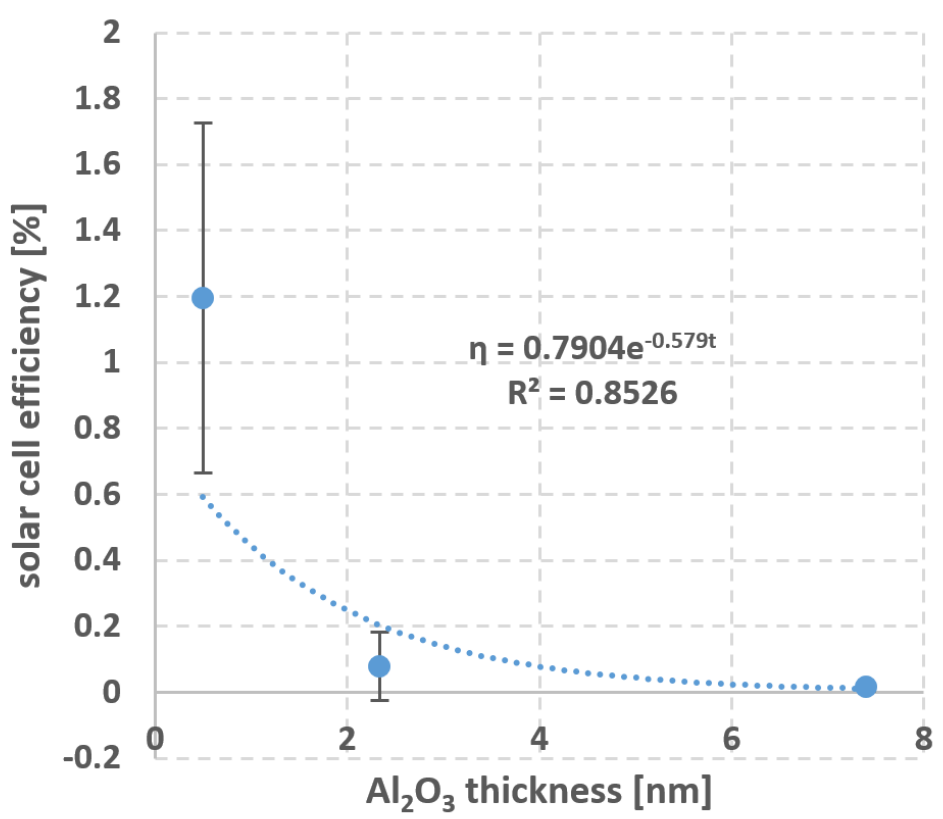

(b)

Figure 4.12: Measured efficiencies of MIS solar cells fabricated on RIE etched $n$ type silicon substrates with ALD deposited $\mathrm{Al}_{2} \mathrm{O}_{3}$ insulator and in situ PEDOT (a) table of calculated efficiencies (b) average measured efficiencies as a function of dielectric thickness. 


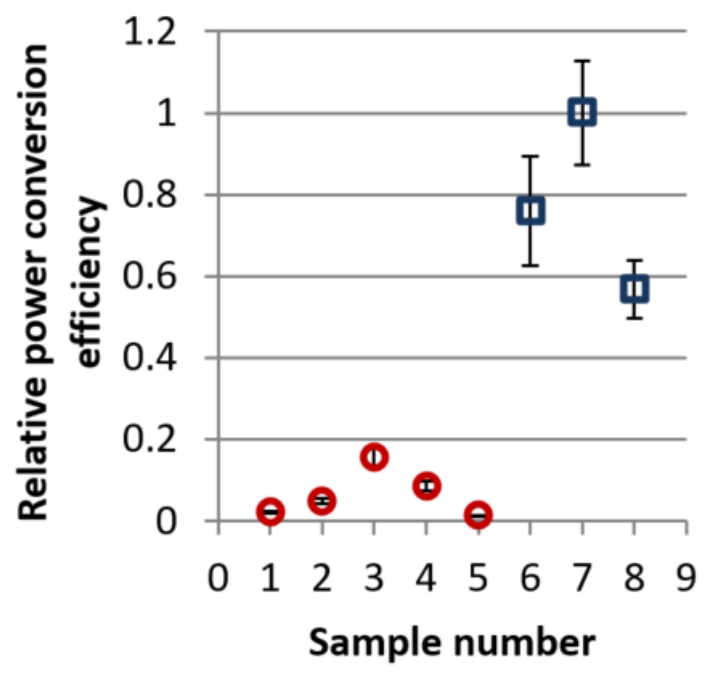

O Flat $\square$ Structured

Figure 4.13: Relative power conversion efficiencies of flat versus nanostructured MIS diodes with ALD deposited $\mathrm{Al}_{2} \mathrm{O}_{3}$ insulator and PEDOT:PSS conductor. The error on the efficiency shown is mostly due to the uncertainty in the incident light intensity, as the irradiation varies widely with lateral lamp alignment as well as its distance away from the sample. 
characteristics of the surface. The added processing complexity of forcing the polymer into the nanostructure and using two different PEDOT formulations for maximizing light transmission to the silicon substrate are surmountable issues that should not be enough of a deterrent to abandon the possibility of increased solar cell efficiency via surface nanostructuring. 


\section{Chapter 5}

\section{Semiconductor Solver}

To assess the effect that replacing a conventional conductor with an organic one has on the operation of the MIS device, two batches of devices were processed in parallel. One set of MIS diodes was topped with Au electrodes. The other set received PEDOT:PSS. Since both of these materials are reported to have very similar work functions (approximately $5.1 \mathrm{eV}$ ) [62,128], a similar operation of the diodes can be expected. Surprisingly, the organic hybrid MIS displayed much lower current and had some features in its IV curve that were absent in the conventional counterpart. To explore the effects of the organic conductor on the MIS operation, a semiconductor solver was written in MATLAB. It was deemed necessary to write custom solver such that a custom, physics-based model for the organic conductor could be used.

\subsection{Physics Covered and Assumptions Made}

Even though the devices of interest were made on $n$-type substrates, the solver was written to accept both $p$ and $n$-type substrates for generality. The simulator solves the drift-diffusion, continuity and Poisson equations for a steady-state solution in one dimension simultaneously and self-consistently with tunneling through the interfacial dielectric. The equations involved are discussed in Chapter 2 are reproduced below 
for convenience.

$$
\begin{gathered}
J_{n}=q n \mu_{n} E+q D_{n} \frac{d n}{d x}=\mu_{n} n \frac{d E_{f n}}{d x} \\
J_{p}=q n \mu_{p} E-q D_{p} \frac{d p}{d x}=\mu_{p} p \frac{d E_{f p}}{d x} \\
\frac{\delta n}{\delta t}=\frac{1}{q} \frac{d J_{n}}{d x}+U_{n}=0 \\
\frac{\delta p}{\delta t}=\frac{1}{q} \frac{d J_{p}}{d x}+U_{p}=0 \\
\epsilon \frac{d^{2} V}{d x^{2}}=-\left(p-n+N_{D}-N_{A}\right) \\
J_{T V}=\int_{-\infty}^{E_{v}} q v_{p} N_{p}\left(f_{s}^{\prime}-f_{m}^{\prime}\right) T_{q} d E \\
J_{T C}=\int_{E_{c}}^{\infty} q v_{n} N_{n}\left(f_{s}-f_{m}\right) T_{q} d E
\end{gathered}
$$

The interface states were included in the simulator. It was determined that defining the Fermi-level through surface recombination velocities yielded a far more stable simulation and easier convergence then equating the tunneling and recombination current components as described in Chapter 2. The Fermi-level for the interface states is determined by setting the interface state occupancy, $f_{s s}$, to 0.5 and solving for energy. The interface state occupancy is defined by equations (5.8) to (5.14), where $f_{s s 0}$ is the interface state occupancy without tunneling current, $f_{m}$ is the conductor occupancy, $\tau_{T}$ is the tunneling time constant, and $\tau_{r}$ is the recombination time constant $[18,129,130]$. 


$$
\begin{gathered}
f_{s s}=\frac{\tau_{T} f_{s s 0}+\tau_{r} f_{m}}{\tau_{T}+\tau_{r}} \\
f_{s s 0}=\frac{n_{s} S_{n}+p_{1} S_{p}}{\left(n_{s}+n_{1}\right) S_{n}+\left(p_{s}+p_{1}\right) S_{p}} \\
S_{n, p}=N_{s s} \sigma_{n, p} v_{t h} \\
p_{1}=N_{V} \exp \frac{E_{V}-E}{k T} \\
n_{1}=N_{C} \exp \frac{E-E_{C}}{k T} \\
\tau_{T}=\tau_{0} T_{q} \\
\tau_{r}=\left[\sigma_{n} v_{t h}\left(n_{s}+n_{1}\right)+\sigma_{p} v_{t h}\left(p_{s}+p_{1}\right)\right]^{-1}
\end{gathered}
$$

Here $n_{s}$ and $p_{s}$ are the surface electron and hole concentrations, $\sigma_{n}$ and $\sigma_{p}$ are the capture cross-sections for electrons and holes, $v_{t h}$ is thermal velocity. $S_{n}$ and $S_{p}$ are termed surface recombination velocities for electrons and holes. $T_{q}$ is the same tunneling probability, as was used in equations (5.6) and (5.7).

The distribution of interface states was implemented using the disorder-induced gap state (DIGS) model as described by equation $(5.15)[37,131]$.

$$
N_{s s}=N_{m i n} e^{\left(\frac{\left|E-E_{H O}\right|}{E_{o j}}\right)^{n_{j}}}
$$

where $j=d$ for donor states below $E_{H O}$ and $j=a$ for acceptor states above $E_{H O}$. The values for surface state distribution parameters, $E_{o j}$ and corresponding exponents, $n_{j}$, are listed in Table 5.1.

The recombination currents between the interface states and conduction and valence bands are defined by [19]

$$
J_{R C}=q \int_{E_{V}}^{E_{C}}-\sigma_{n} v_{t h} N_{s s}\left(f_{s s} n_{1}-\left(1-f_{s s}\right) n_{s}\right) d E
$$




$$
J_{R V}=q \int_{E_{V}}^{E_{C}}-\sigma_{p} v_{t h} N_{s s}\left(f_{s s} p_{s}-\left(1-f_{s s}\right) p_{1}\right) d E
$$

These equations fully describe the current flow and the potential throughout the MIS diode. The recombination, included in the continuity equations (5.3) and (5.4), was modelled as Schokley-Read-Hall recombination, described below. However, since the substrates used in device fabrication were high quality Czochralski wafers, recombination was not expected to play a large role in device operation.

Solar charge generation was included as well to examine the effect of stray low intensity illumination during measurement, however, the same analysis can be used for any optical carrier generation. The generation rate was calculated as shown below with the solar spectrum, $S_{\text {solar }}$, and attenuation coefficients, $\alpha$, for silicon obtained from PVEducation [9].

$$
G=\frac{\frac{d}{d x}\left[e^{-\alpha x} \int S_{s o l a r} d \lambda\right]}{h c}
$$

Contact resistance was included to account for the effect of non-idealities in the device contacts and the measurement setup and was implemented as a dilation of the voltage axis post-simulation.

$$
V_{\text {measurement }}=V_{\text {simulation }}+R_{c} I_{\text {total }}
$$

Tunneling though the silicon barrier created by band-bending was added into the simulator but was found to have no effect on the current-voltage characteristic in the temperature range of interest. A few higher-order effects have not been accounted for by the simulation as follows.

Even though it is possible to vary the temperature in the simulation, the variation 
of the semiconductor bandgap with temperature was not accounted for. As the polymeric conductor has a relatively small temperature range of operation, the bandgap variation over this small temperature range was deemed insignificant. The electric field dependence of the electron and hole mobilities was also ignored.

The parameter values used in the simulations are listed in Table 5.1.

\subsection{Methods and Algorithms}

The simulator was written to implement the finite element method. The top contact and the oxide are not meshed, however. The tunneling calculation simply uses the potentials on either side of the insulator.

The semiconductor mesh is limited at the top end by the Debye length in the material and at the lower end by numerical error. Implementing a dynamically adjustable non-uniform mesh over the length of the device would help alleviate the numerical error resulting from this very tight mesh. However, since simulator development was not intended to be the main focus of this thesis and given the complexity of implementation of a dynamic mesh, a static uniform mesh was implemented instead.

The drift-diffusion and continuity equations were linearized using the SharfetterGummel approach, where the variation in the carrier concentration between mesh points was assumed to be exponential. This treatment allows for a much faster convergence. It results in the following expressions for electron and hole drift-diffusion current densities:

$$
\begin{array}{r}
J_{n}=\frac{q \mu_{n} V_{t}}{2 \Delta}\left\{\left[n_{j} B\left(\frac{\psi_{j}-\psi_{j-1}}{V_{t}}\right)-n_{j-1} B\left(\frac{\psi_{j-1}-\psi_{j}}{V_{t}}\right)\right]\right. \\
\left.+\left[n_{j+1} B\left(\frac{\psi_{j+1}-\psi_{j}}{V_{t}}\right)-n_{j} B\left(\frac{\psi_{j}-\psi_{j+1}}{V_{t}}\right)\right]\right\}
\end{array}
$$




\begin{tabular}{|c|c|c|}
\hline \multicolumn{3}{|c|}{ Universal Constants } \\
\hline c & speed of light & $3 \times 10^{8} \mathrm{~m} / \mathrm{s}$ \\
\hline $\mathrm{q}$ & electron charge & $1.60217657 \times 10^{-19} \mathrm{C}$ \\
\hline $\mathrm{k}_{B}$ & Boltzmann's constant & $1.381 \times 10^{-34} \mathrm{Js}$ \\
\hline$\epsilon_{o}$ & permittivity of free space & $8.854 \times 10^{-14} \mathrm{~F} / \mathrm{cm}$ \\
\hline \multicolumn{3}{|c|}{ Substrate Properties } \\
\hline $\mathrm{L}_{\max }$ & substrate thickness & $279 \mu \mathrm{m}$ \\
\hline$\chi_{S i}$ & electron affinity of silicon & $4.05 \mathrm{eV}$ \\
\hline$\epsilon_{S i}$ & permittivity of silicon & $11.7 \epsilon_{o}$ \\
\hline$E_{G, S i}$ & bandgap of silicon & $1.12 \mathrm{eV}$ \\
\hline$N_{C}$ & density of states in the & $2.82 \times 10^{19} \mathrm{~cm}^{-3}$ \\
\hline$N_{V}$ & $\begin{array}{l}\text { conduction band of silicon } \\
\text { density of states in the } \\
\text { valence band of silicon }\end{array}$ & $1.83 \times 10^{19} \mathrm{~cm}^{-3}$ \\
\hline$\mu_{n}$ & electron mobility in silicon & $1350 \mathrm{~cm}^{2} / \mathrm{Vs}$ \\
\hline$\mu_{p}$ & hole mobility in silicon & $470 \mathrm{~cm}^{2} / \mathrm{Vs}$ \\
\hline \multicolumn{3}{|c|}{ Interface States Model } \\
\hline$E_{H O}-E_{V}$ & charge neutrality level & $0.32 \mathrm{eV}$ \\
\hline$E_{o d}, n_{d}$ & donor model parameters & $0.17,1.8$ \\
\hline$E_{o a}, n_{a}$ & acceptor model parameters & $0.48,4.5$ \\
\hline$\sigma_{n, p}$ & charge capture cross-sections & $5 \times 10^{16} \mathrm{~cm}^{2}$ \\
\hline$\tau_{0}$ & nominal tunneling time constant & $10^{-13} \mathrm{~s}$ \\
\hline \multicolumn{3}{|c|}{ Insulator Properties } \\
\hline$\chi_{o x}$ & electron affinity of $\mathrm{SiO}_{2}$ & $0.9 \mathrm{eV}$ \\
\hline$\epsilon_{o x}$ & permittivity of $\mathrm{SiO}_{2}$ & $3.9 \epsilon_{o}$ \\
\hline$E_{G, o x}$ & bandgap of $\mathrm{SiO}_{2}$ & $9 \mathrm{eV}$ \\
\hline
\end{tabular}

Table 5.1: Parameters used in device simulations. 


$$
\begin{array}{r}
J_{p}=\frac{q \mu_{p} V_{t}}{2 \Delta}\left\{\left[p_{j} B\left(\frac{\psi_{j-1}-\psi_{j}}{V_{t}}\right)-p_{j-1} B\left(\frac{\psi_{j}-\psi_{j-1}}{V_{t}}\right)\right]\right. \\
\left.+\left[p_{j+1} B\left(\frac{\psi_{j}-\psi_{j+1}}{V_{t}}\right)-p_{j} B\left(\frac{\psi_{j+1}-\psi_{j}}{V_{t}}\right)\right]\right\}
\end{array}
$$

Here $V_{t}$ is the thermal voltage defined as $k_{B} T / q, \mu_{n}$ and $\mu_{p}$ are electron and hole mobilities respectively, $\Delta$ is the mesh spacing (assuming a uniform mesh), $\psi$ is the potential, $j$ enumerates the mesh points, and $B$ is the Bernoulli function defined as

$$
B(x)=\frac{x}{e^{x}-1}
$$

When solving the Poisson equation, the change in potential, $\delta$, is solved for instead of the new potential:

$$
-\frac{2}{\Delta^{2}} \delta_{j-1}+\left(\frac{2}{\Delta^{2}}+\frac{2}{\Delta^{2}}+n_{j}+p_{j}\right) \delta_{j}-\frac{2}{\Delta^{2}} \delta_{j+1}=\psi_{j}^{\prime \prime}+N_{j}-n_{j}+p_{j}
$$

When solving for the electron and hole carrier densities, the recombination rate is treated implicitly as shown in equation (5.24) and (5.25). This approach makes the algorithm far more stable, allowing for treatment of real-sized devices.

$$
\begin{array}{r}
\frac{V_{t} \mu_{p}}{\Delta^{2}} B\left(\frac{\psi_{j}-\psi_{j-1}}{V_{t}}\right) p_{j-1} \\
-\left[\frac{V_{t} \mu_{p}}{\Delta^{2}} B\left(\frac{\psi_{j+1}-\psi_{j}}{V_{t}}\right)+\frac{V_{t} \mu_{p}}{\Delta^{2}} B\left(\frac{\psi_{j-1}-\psi_{j}}{V_{t}}\right)+\frac{n_{j}}{D}\right] p_{j} \\
+\frac{V_{t} \mu_{p}}{\Delta^{2}} B\left(\frac{\psi_{j}-\psi_{j+1}}{V_{t}}\right) p_{j+1}=-\frac{1}{D}
\end{array}
$$




$$
\begin{array}{r}
\frac{V_{t} \mu_{n}}{\Delta^{2}} B\left(\frac{\psi_{j-1}-\psi_{j}}{V_{t}}\right) n_{j-1} \\
-\left[\frac{V_{t} \mu_{n}}{\Delta^{2}} B\left(\frac{\psi_{j}-\psi_{j+1}}{V_{t}}\right)+\frac{V_{t} \mu_{n}}{\Delta^{2}} B\left(\frac{\psi_{j}-\psi_{j-1}}{V_{t}}\right)+\frac{p_{j}}{D}\right] n_{j} \\
+\frac{V_{t} \mu_{n}}{\Delta^{2}} B\left(\frac{\psi_{j+1}-\psi_{j}}{V_{t}}\right) n_{j+1}=-\frac{1}{D} \\
D=\tau_{p}\left(n_{j}+n_{\text {intrinsic }}\right)+\tau_{n}\left(p_{j}+p_{\text {intrinsic }}\right)
\end{array}
$$

To decouple this set of differential equations, the Poisson, drift-diffusion, and continuity equations were solved iteratively, using the previous solution as a starting point until a convergence criteria was met, as shown in the flow chart in Figure 5.1.

The ohmic boundary condition at the back contact was maintained by enforcing the equilibrium solution there, while the boundary condition at the Si/insulator interface is set by equating the tunneling and drift-diffusion currents at that point for both electrons and holes. Considering that over the range of operation of interest $(-1 \mathrm{~V}$ to $1 \mathrm{~V})$, the limiting conduction mechanism is likely to transition between tunnel-limited and drift-diffusion limited, the most reliable way to ensure current continuity in the simulation was found to be the graphing method illustrated in Figure 5.2.

The added benefit of using the graphing method to establish the boundary condition is the increased tolerance to applied voltage steps. Since a range of potentials is examined when establishing the boundary condition, much larger voltage steps can be taken, which speeds up the simulation considerably.

The complete code for the simulator can be found in Appendix G. 


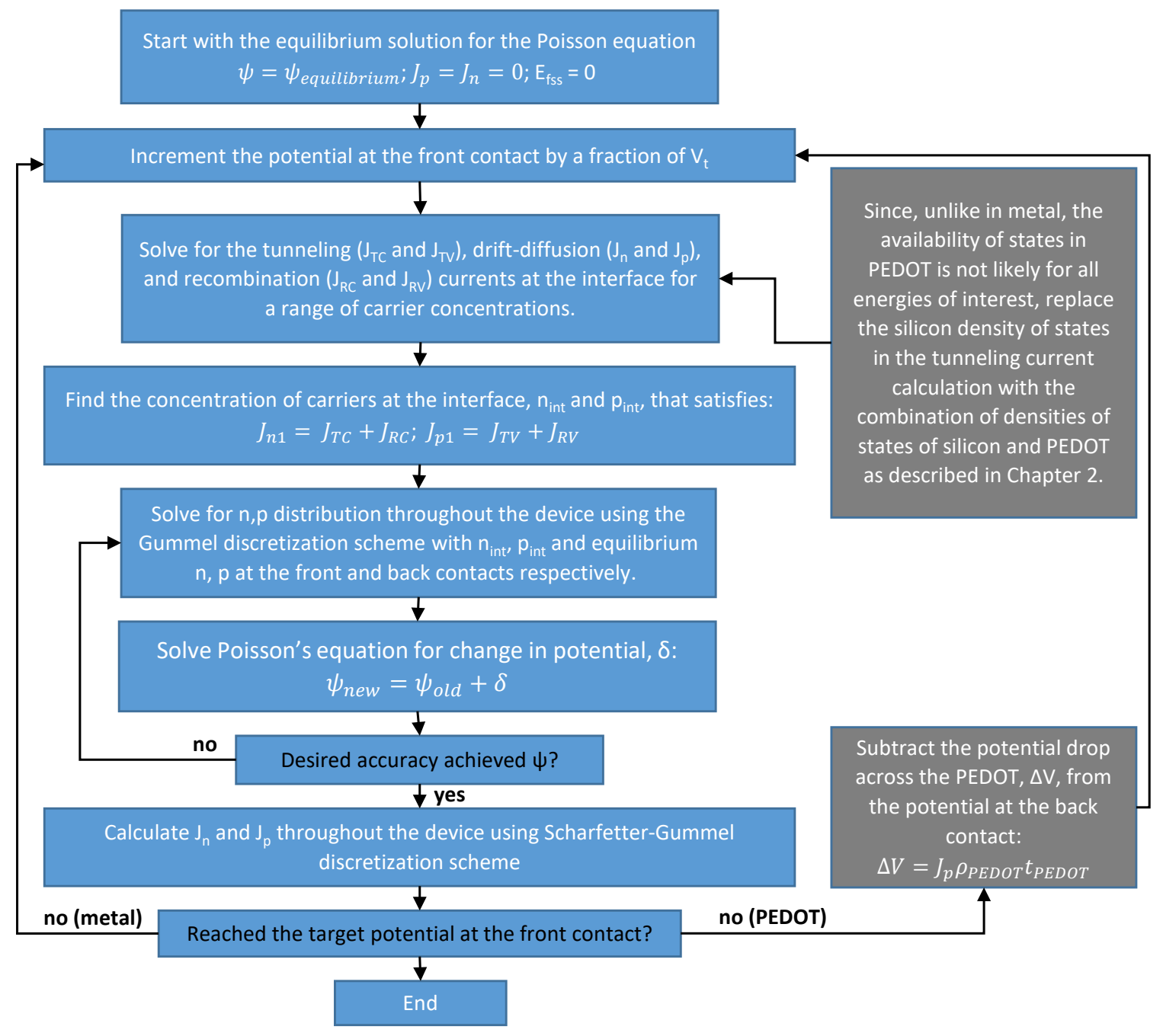

Figure 5.1: Algorithm used by the simulator to solve the continuity equation. Modifications included in the model for PEDOT are highlighted in gray. 


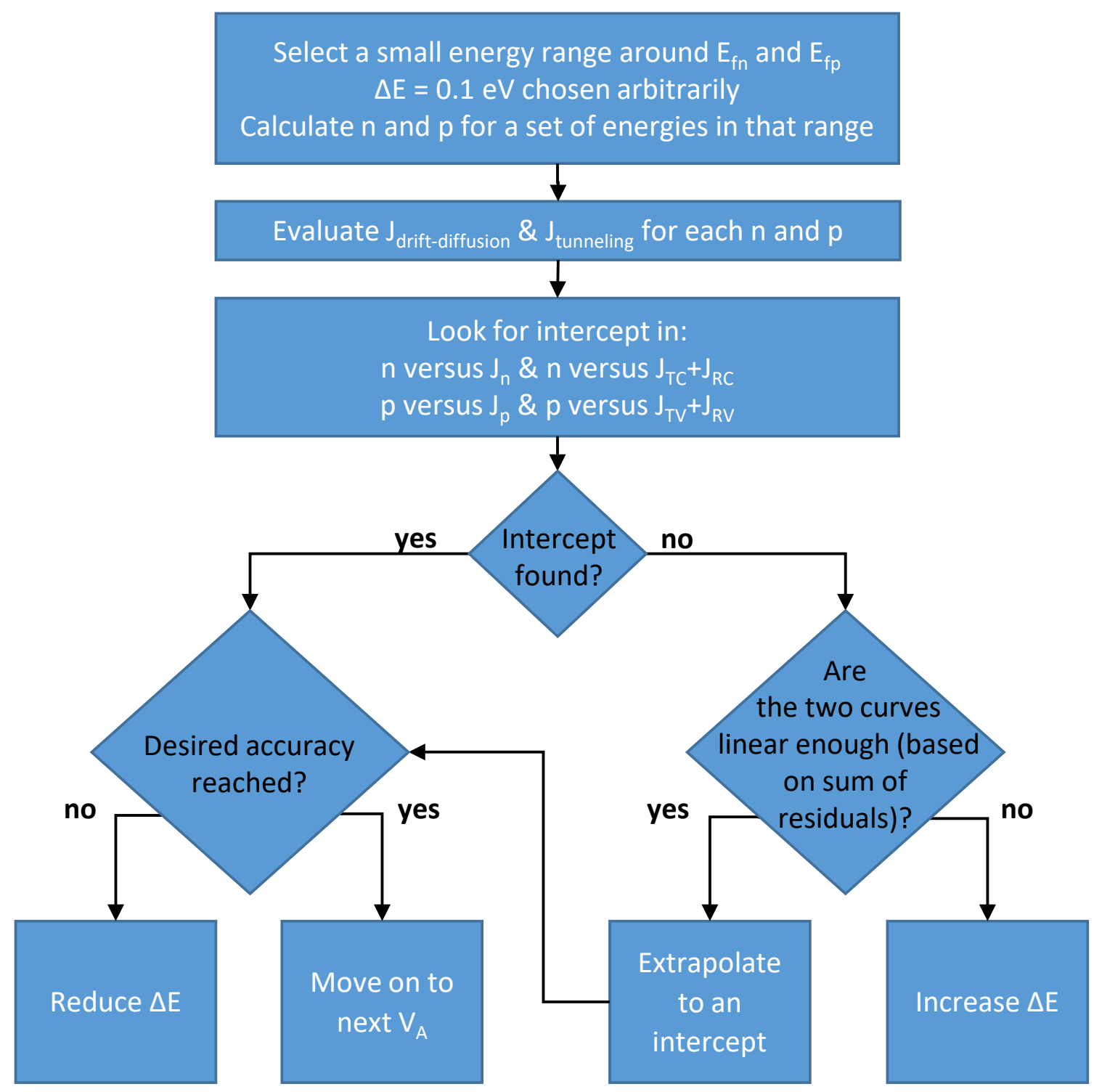

Figure 5.2: Algorithm for establishing the substrate/insulator boundary condition. 


\subsection{Verification of the Simulator}

The operation of the simulator was verified by simulating a Schottky diode without an insulator and with a conventional metal contact, and comparing the results with the analytic model. The current-voltage characteristic of a Schottky diode obeys the following relationship [8]

$$
J=A^{* *} T^{2} e^{-\frac{q \phi_{B n}}{n k_{B} T}}\left[e^{\frac{q\left(V-I R_{S}\right)}{n k_{B} T}}-1\right]
$$

Where $A^{* *}$ is the effective Richardson constant and can be taken to be $110 \mathrm{~A} / \mathrm{cm}^{2} \mathrm{~K}^{2}$ [8]. $T$ is the temperature, $n$ is the ideality factor, and $\phi_{B n}$ is the potential barrier between the metal contact and the substrate. It is defined as follows [8]

$$
\phi_{B n}=\phi_{m}-\chi_{S i}+\frac{k_{B} T}{q}
$$

The series resistance comes from the bulk of the substrate and is calculated assuming a $1 \mathrm{~cm}^{2}$ device based on the substrate doping. As in the case of the contact resistance, it is applied to the analytic solution as a dilation of the voltage axis.

$$
R_{S}=\frac{\left(q \mu_{n} N_{D}\right)^{-1} L_{\max }}{A}
$$

where $L_{\max }$ is the thickness of the substrate and $A$ is the area of the diode.

Figure 5.3 shows the comparison of the simulated diodes without the tunneling dielectric and the analytical solutions for the same devices calculated as per equations (5.27)-(5.29). The agreement is excellent with the high voltage roll-off completely accounted for by the series resistance of the substrate as expected.

The simulations discussed above took about 30 seconds to complete on an Intel Xeon X5690 processor with a convergence criteria of $0.1 \%$ precision on the current 


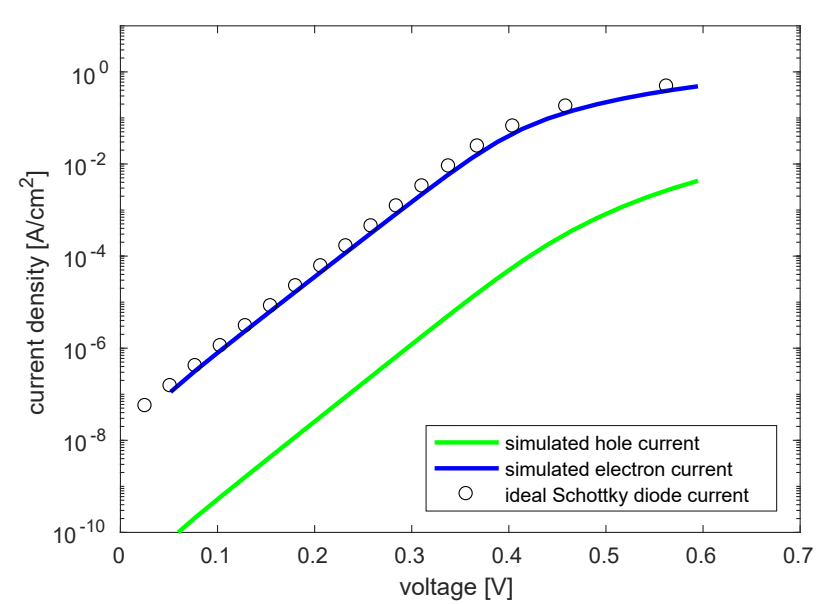

(a)

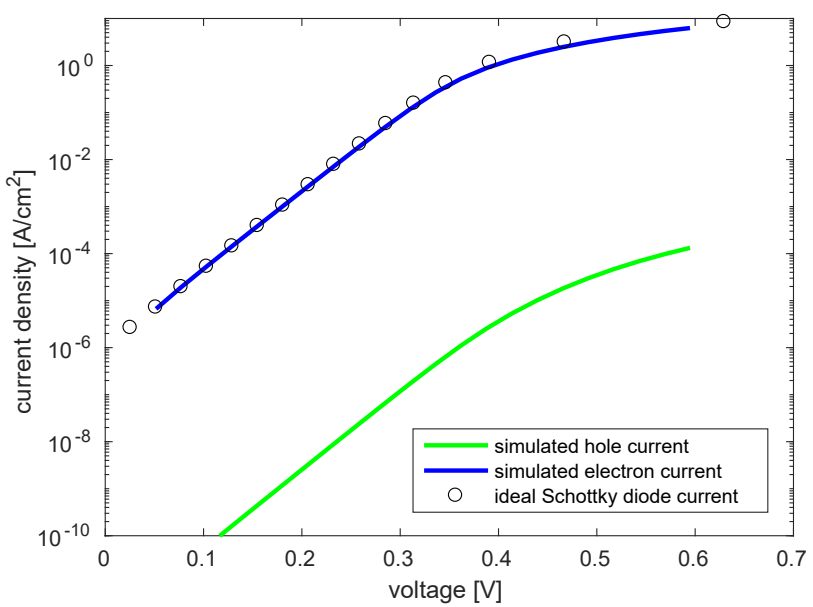

(b)

Figure 5.3: Comparison of simulated and calculated current-voltage characteristics of Schottky diodes (a) $N_{D}=5 \times 10^{14} \mathrm{~cm}^{-3}, \phi_{m}=4.9 \mathrm{eV}$ (b) $N_{D}=5 \times 10^{15} \mathrm{~cm}^{-3}, \phi_{m}=4.8 \mathrm{eV}$.

density values. The simulator was successfully tested in the range of $-0.5 \mathrm{~V}$ to $1.5 \mathrm{~V}$ for the applied voltage and $4.3 \mathrm{eV}$ to $5.1 \mathrm{eV}$ for the top contact work function. 


\section{Chapter 6}

\section{PEDOT versus Au}

To explore the effects of replacing a traditional metal with an organic conductor, two types of devices have been manufactured in parallel: $\mathrm{Au} / \mathrm{SiO}_{2} / \mathrm{Si}$ and PEDOT:PSS $/ \mathrm{SiO}_{2} / \mathrm{Si}$, both on flat substrates with thermally-grown oxide. If PEDOT is to be treated as a metal, which is routinely done in the literature at present, the two devices should show very similar electrical characteristics, as the work functions of both $\mathrm{Au}$ and PEDOT:PSS are generally reported to be alike (around 5.1$5.2 \mathrm{eV})[62,128]$. Our measurements, however, reveal higher current densities in the traditional Au-topped junction as compared to the PEDOT-topped device. To understand the difference in operation of these otherwise identical structures the effects of the qualities of PEDOT:PSS that make it inherently different from an ideal metal were investigated using a custom-built simulator. This simulator is discussed in detail in Chapter 5. In particular variable-range hopping conduction in the PEDOT and the inclusion of the polaron and bipolaron bands in the bandgap of PEDOT:PSS were investigated. The limited availability of carrier states in PEDOT:PSS due to the finite width of the energy bands and the alignment of the energy bands were found to have the most significant effect on the operation of PEDOT:PSS $/ \mathrm{SiO}_{2} / \mathrm{Si}$ devices. 


\subsection{Processing}

To generate comparable devices where the only difference is in the top conductor used, such that the effects of gold versus PEDOT:PSS on the operation of an MIS device can be assessed, two wafers, designated Au and PEDOT, were processed in parallel. The starting wafers were $\mathrm{n}(100)$ 1-10 $\Omega \mathrm{cm}$ from the same batch with $100 \mathrm{~nm}$ of thermal oxide already pre-grown.

To open windows in the oxide for the placement of the diodes, the wafers were treated in PlasmaPreen oxygen plasma followed by a vapour prime in HMDS. A mask of apertures was photolithographically transferred into a photoresist on the surface to define diode placement and geometry. Oxide was etched in buffered HF to hydrophobia. This etch also has the added benefit of removing any unwanted native oxide on the back of the wafer. The photoresist was stripped in a PlasmaPreen.

Prior to back metallization, the wafers were RCA cleaned ending with $1 \% \mathrm{HF}$. The back contact was formed by depositing $250 \mathrm{~nm}$ of $\mathrm{Al}$ by e-beam evaporation.

Prior to oxidation of the front surface for the formation of the interfacial tunneling oxide, the wafers were flash-dipped in $1 \% \mathrm{HF}$ to remove any native oxide. The oxide was thermally-grown at $500^{\circ} \mathrm{C}$ for $10 \mathrm{~min}$ for a target oxide thickness in the range of $1-2 \mathrm{~nm}$.

PEDOT:PSS (Clevios PH1000 supplied by H.C. Starck) was spin-cast at $1000 \mathrm{RPM}$ with acceleration of $180 \mathrm{RPM} / \mathrm{s}$ on top of the PEDOT wafer to create an MIS contact. These devices were measured by probing the PEDOT directly. To electrically isolate the diode under measurement, the PEDOT:PSS around the diode was scratch-patterned with a dull probe tip as shown in Figure 6.1(a).

The Au wafer, on the other hand, received a coating of gold, thermally-evaporated through a shadow mask aligned to the diode openings in the oxide as shown in Figure 


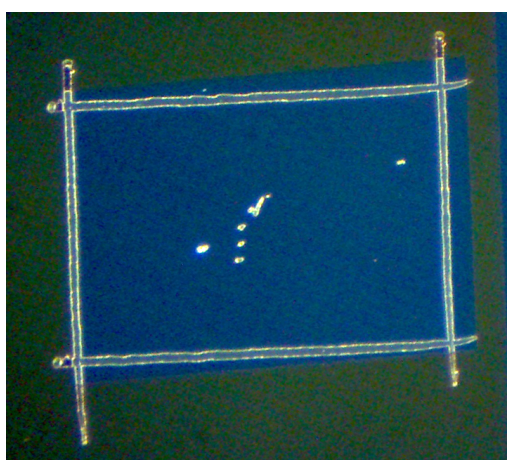

(a)

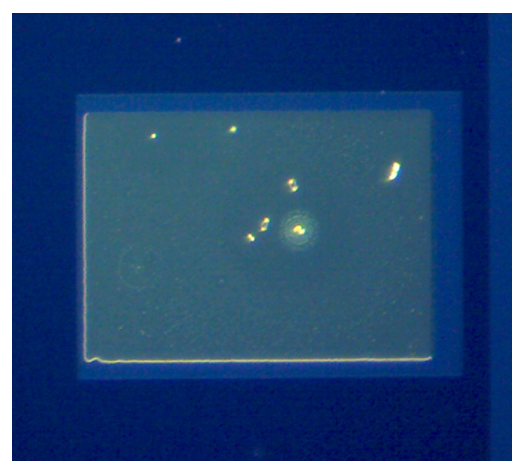

(b)

Figure 6.1: Samples of top conductor patterning for hybrid and inorganic diodes. The light blue window is the opening in the isolating $100 \mathrm{~nm}$ oxide, where only a thin tunneling oxide is present. The materials are pattered such that there is no overlap with the thick oxide. The markings in the middle of the pattered squares are spots where the probe was landed.(a) Example of scratch-patterned PEDOT. (b) Example of pattered Au.

6.1(b). Thus the $\mathrm{Au} / \mathrm{SiO}_{2} / \mathrm{Si}$ devices were already electrically isolated from one another on the wafer and did not need any additional processing prior to measurement.

\subsection{Comparison}

The electrical characteristics were measured for several devices of each type: conventional inorganic MIS with gold and organic hybrid MIS with PEDOT:PSS. Figure 6.2 shows the average current-voltage characteristics of the two types of devices for comparison.

The ideality factor, $n$, (as defined in Section 5.3) for the inorganic $\mathrm{Au} / \mathrm{SiO}_{2} / \mathrm{Si}$ diode was extracted from the IV characteristic at biases below $0.25 \mathrm{~V}$ to be 1.03 , which confirms drift-diffusion limited nature of the current at those biases, confirming minority carrier diode operation. In the hybrid PEDOT:PSS/SiO$/ 2 / \mathrm{Si}$ diode this portion of IV curve is obscured by what looks like a shunt resistance.

The two main differences between the inorganic and hybrid diodes to consider are 


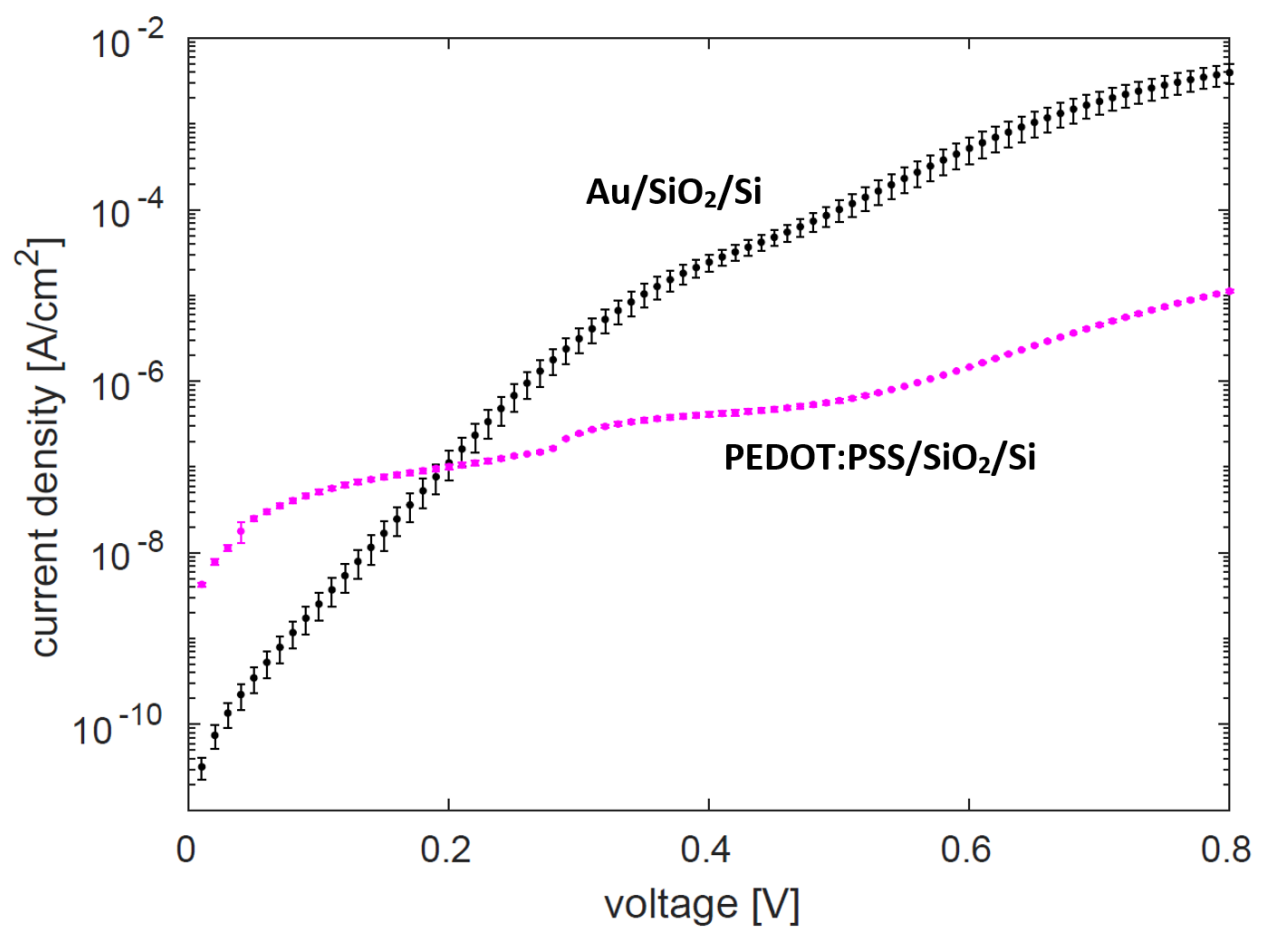

Figure 6.2: Average dark IV characteristics of several inorganic $\mathrm{Au} / \mathrm{SiO}_{2} / \mathrm{Si}$ and hybrid PEDOT:PSS $/ \mathrm{SiO}_{2} / \mathrm{Si}$ diodes. The error bars represent the standard deviations in the measured current values of several identical devices. 
the obvious disparity in the magnitude of the current density of one to three orders of magnitude. The other difference is the relative magnitude of current density above and below $0.5 \mathrm{~V}$. The ratio of current densities taken at $0.8 \mathrm{~V}$ and $0.4 \mathrm{~V}$ is 162 for gold-topped diodes and only 27 for diodes with PEDOT:PSS.

\subsection{Difference in Current Densities}

A thicker tunneling barrier in the hybrid diode as compared to the inorganic one could account for the difference in current densities observed. However, since the two wafers were oxidised together in the same run it is not likely to be due to processing. It is possible, though, that some residual water in the PEDOT:PSS, which is known to linger or be re-absorbed from the atmosphere post baking [62], could migrate to the Si surface and continue oxidising it. Alternatively, an accumulation of non-conductive PSS layer at the polymer $/ \mathrm{SiO}_{2}$ interface can effectively increase the tunneling barrier. This phenomenon is deemed to be responsible for the electron blocking property of PEDOT:PSS [132]. This process, however, does not account for slower current density increase of the hybrid, PEDOT:PSS-topped device.

A limited number of energy states for the carriers to go to in the PEDOT:PSS, as compared to $\mathrm{Au}$, can explain both the reduced current density in the PEDOTcontaining devices as well as its slower current density increase seen in the currentvoltage characteristic (Figure 6.2). Figure 6.3(a) shows the band diagram of an MIS diode with gold as the top contact. There are a virtually unlimited number of energy states available for holes to populate below the metal work function level and just as many states available for electrons above it. Thus, the probability of charge transfer from the silicon substrate to gold is practically limited (aside from the oxide thickness) only by the occupancy of states in silicon. Often this same model is used for PEDOT:PSS as well. Unlike a uniform three-dimensional lattice of Au atoms, 


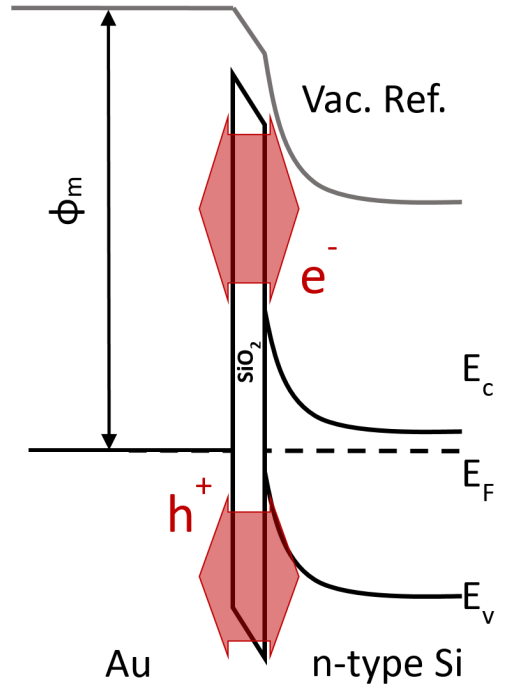

(a)

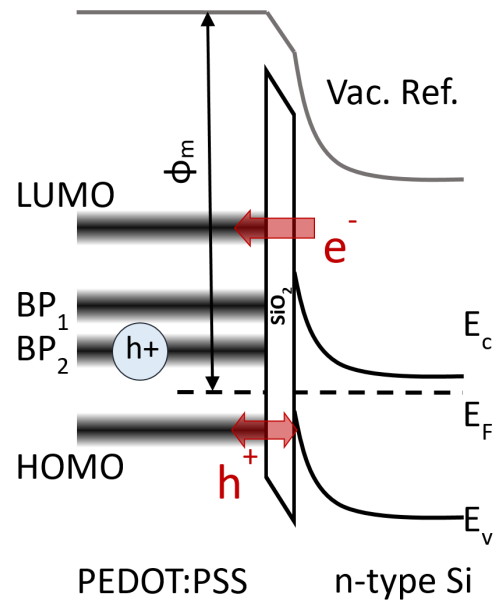

(b)

Figure 6.3: Energy band diagrams for (a) an inorganic $\mathrm{Au} / \mathrm{SiO}_{2} / \mathrm{Si}$ diode (b) a hybrid PEDOT:PSS $/ \mathrm{SiO}_{2} / \mathrm{Si}$ diode. The thin energy bands of the conductive polymer may limit the current density in the hybrid diode. No such limitation exists in the inorganic device.

PEDOT:PSS is comprised of relatively short polymer chains, that can only support one-dimensional charge transport ${ }^{1}[61,62]$. This limits the width of the energy bands as shown in Figure 6.3(b). Between the chains, the charge is limited to a thermallyactivated hopping mechanism, which does not contribute to the band model. The degree of alignment and proximity of the polymer chains determine the thickness of the effective bands available for charge transport [61].

In a 3D material, such as a silicon substrate, the density of states (DOS) is well understood and follows a parabolic model as shown in Figure 6.4(a). In the case of a conventional metal, that has no bandgap, the edges of the bands are very far away from the substrate bandgap. As a result, the density of states in the metal in the vicinity of the silicon substrate bandgap is very high and does not limit charge transfer to and from silicon. The bands in PEDOT, on the other hand are quite limited in

\footnotetext{
${ }^{1}$ Two-dimensional or non-integral (between 1D and 2D) transport has been proposed for systems where the polymer chains are well aligned [61,62]
} 


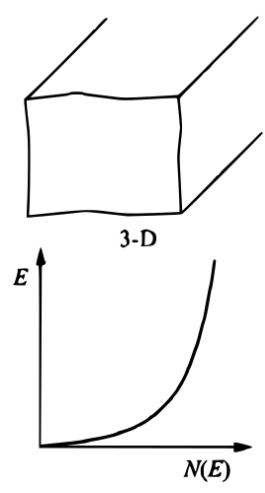

(a)

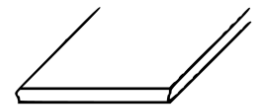

2-D

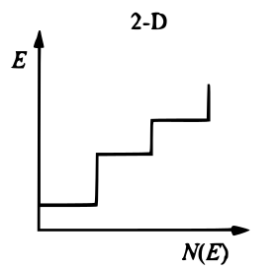

(b)

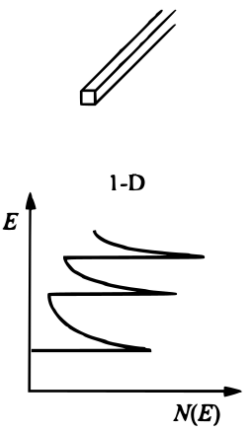

(c)

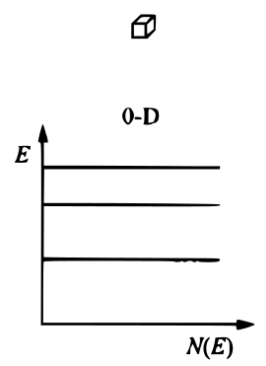

(d)

Figure 6.4: Density of states (DOS) for materials of different dimensionality. After $[8]$.

energy. Therefore, the number of states available for charge transfer in PEDOT and not in silicon may limit the possible transitions in this case (Figure 6.3(b)). Since it is unclear whether PEDOT behaves as a $3 \mathrm{D}, 2 \mathrm{D}$, or a $1 \mathrm{D}$ material, the shape of the density of states is unknown. In simulation various shapes of DOS for PEDOT:PSS were considered (Figure 6.5). If PEDOT behaves as a 3D conductor, the density of states could be approximated as two parabolic sections. It was arbitrarily decided that the DOS hole and electron effective masses would be the same, thus the two parabolic sections would meet in the middle of the band as shown in Figure 6.5(a) If PEDOT behaves as a 1D conductor, the parabolic DOS would terminate abruptly at a band edge as illustrates in Figure 6.5(b). Gaussian DOS is often used for hopping mobility calculations (Figure 6.5(c)) [61]. The width of the bands in an organic conductor is expected to be in the range of $0.1 \mathrm{eV}-0.4 \mathrm{eV}$ [61]. Even a rectangular DOS was tried (Figure 6.5(d)). However, the simulations show that the shape plays little to no role in the current-voltage characteristics of the diode. The widths of the bands and their alignment relative to the Fermi level in PEDOT determine the magnitude of the current density as well as its shape. As such, since Gaussian DOS are easy to implement and manipulate mathematically, and since there is a precedent 


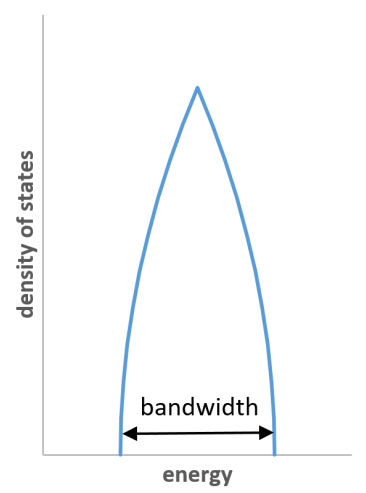

(a) 3D DOS

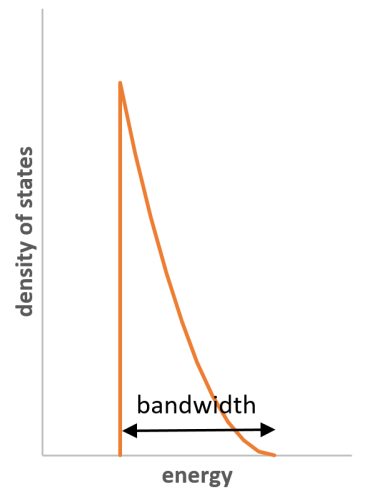

(b) $1 \mathrm{D}$ DOS

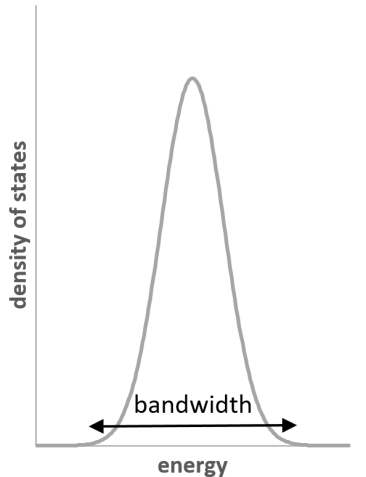

(c) Gaussian

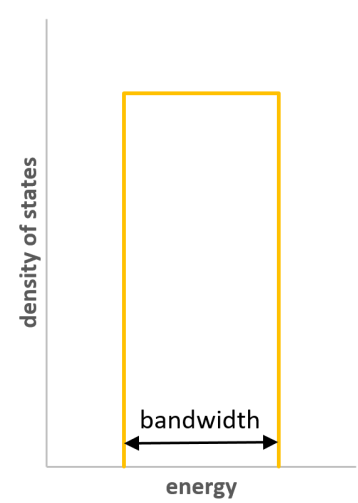

(d) Rectangular

Figure 6.5: Density of states used for PEDOT in simulation.

of their use in the literature for organic conductors [61], that was the model used.

In inorganic semiconductors, the energy band structure of interest as it pertains to the electronic properties of the material consists of semi-infinite conduction and valence bands separated by an energy gap. In organic conductors, such as PEDOT the picture is slightly different. Not only are the bands limited in energy, but the optical absorption spectra of PEDOT:PSS shows emergence of other energy bands as the material becomes more heavily doped. These bands are thought to be associated with the physical distortion of the polymer chain once it is oxidised, and are termed polaron and bipolaron bands as illustrated in Figure 6.6(b) [62]. Thus a picture emerges showing distinct differences between the electronic structure of gold and PEDOT as illustrated in Figure 6.3

\subsection{Simulated versus Measured Data}

The $\mathrm{Au} / \mathrm{SiO}_{2} / \mathrm{Si}$ and PEDOT:PSS $/ \mathrm{SiO}_{2} / \mathrm{Si}$ devices were simulated in an attempt to match the measured data. The key parameters swept in the simulations were the work function of the metal, $\phi_{m}$, and the oxide thickness, $t_{o x}$. The doping density of the substrate, $N_{D}$, was fixed at the values measured with four-point-probe for the two 


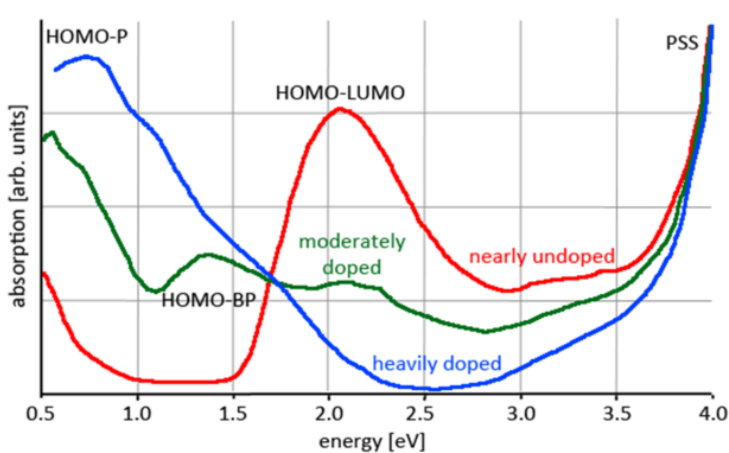

(a)

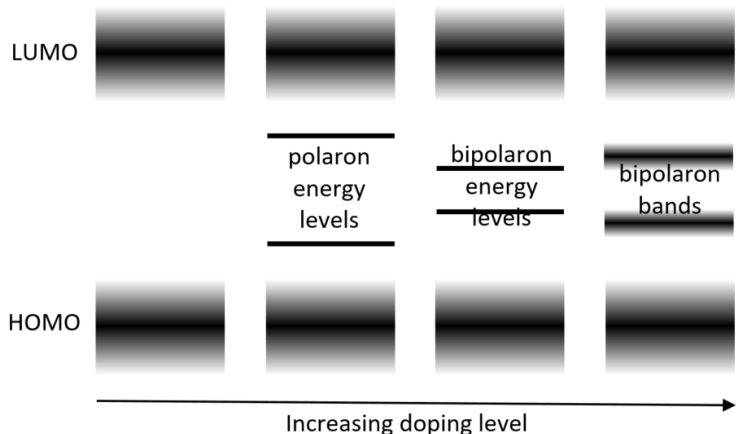

(b)

Figure 6.6: Origin of PEDOT band structure (a) PEDOT:PSS absorption spectrum [62] (b) possible PEDOT:PSS band structure with various doping levels after ref. $[62]$.

sets of devices. Other constants and relevant variables and their values are defined in Chapter 5 .

\subsection{1 $\mathrm{Au} / \mathrm{SiO}_{2} / \mathrm{Si}$}

The resistivity of the starting wafer was measured with a four-point probe to be $6.9 \Omega \mathrm{cm} \pm 0.3 \Omega \mathrm{cm}$, which corresponds to $(6.7 \pm 0.3) \times 10^{14} / \mathrm{cm}^{3}$.

The work function of pristine gold is reported as $\phi_{m}=5.1-5.47 \mathrm{eV}$ [128]. However, the metal work function, as seen by the silicon surface can be heavily influenced by the processing of the surface, and has been reported to vary in the range of $\phi_{m}=4.7$ $5.4 \mathrm{eV}$ [133-135]. The work function of gold on a bare silicon surface was extracted from a capacitance-voltage measurement to be $4.93 \mathrm{eV} \pm 0.02 \mathrm{eV}$. Thus, the work function was swept in the range of $4.7 \mathrm{eV}$ to $5.5 \mathrm{eV}$.

The oxide thickness is the least constrained parameter in this experiment. Attempts were made to measure it with the Plasmos $632 \mathrm{~nm}$ multiangle ellipsometer (model SD2000). However, there was too much variability in the resultant data to deem it reliable. As for the capacitance-voltage measurements that are routinely used 
to assess the thickness of gate oxide in a metal-oxide-semiconductor field effect transistor (MOSFET), the large current leakage which is well expected in an MIS device makes the reliability of such parameter extraction questionable as well. As such the oxide thickness was swept in the range of $t_{o x}=0.5$ to $5 \mathrm{~nm}$, which would cover the range of oxide thicknesses that are practically useful for creating an efficient (or at least a functional) MIS diode. The MIS structure proved to be very sensitive to the oxide thickness in simulation, as expected. The thickness that provided the closest match to the measured data was assumed to be correct.

The best fit for the measured data for the $\mathrm{Au} / \mathrm{SiO}_{2} / \mathrm{Si}$ devices, shown in Figure 6.7, was found with the following parameters: $N_{D}=6.71 \times 10^{14} \mathrm{~cm}^{-3}, \phi_{m}=4.8 \mathrm{eV}$, $t_{o x}=1.65 \mathrm{~nm}$. The distribution of surface states used in this simulation is shown in Figure 6.8. To get a good match to the measured data it is necessary to be able to adjust the electron and hole current components individually. This can be achieved by varying the effective masses of electrons and holes in the oxide barrier. The common values reported in the literature vary between $0.3 \mathrm{~m}_{e}$ and $0.4 \mathrm{~m}_{e}$, where $\mathrm{m}_{e}$ is free-space mass of electron. According to Brar a mass of $0.3 \mathrm{~m}_{e}$ corresponds to a parabolic model for the E-k relationship in the tunneling barrier and a mass of $0.4 \mathrm{~m}_{e}$ is extracted from a non-parabolic (two-band) model $[136,137]$. For the best fit to our data values of $0.4 \mathrm{~m}_{e}$ and $0.3 \mathrm{~m}_{e}$ were used for electrons and holes respectively.

\subsubsection{PEDOT:PSS $/ \mathrm{SiO}_{2} / \mathrm{Si}$}

The resistivity of the starting wafer was measured with a four-point probe to be $6.7 \Omega \mathrm{cm} \pm 0.3 \Omega \mathrm{cm}$, that corresponds to $(7.0 \pm 0.3) \times 10^{14} / \mathrm{cm}^{3}$.

Since the work function of PEDOT:PSS is expected to be similar to that of gold, the value of $\phi_{m}=4.8 \mathrm{eV}$ from the $\mathrm{Au} / \mathrm{SiO}_{2} / \mathrm{Si}$ simulation was a good starting point.

Ideally the oxide thickness for these devices would be the same as in the case 


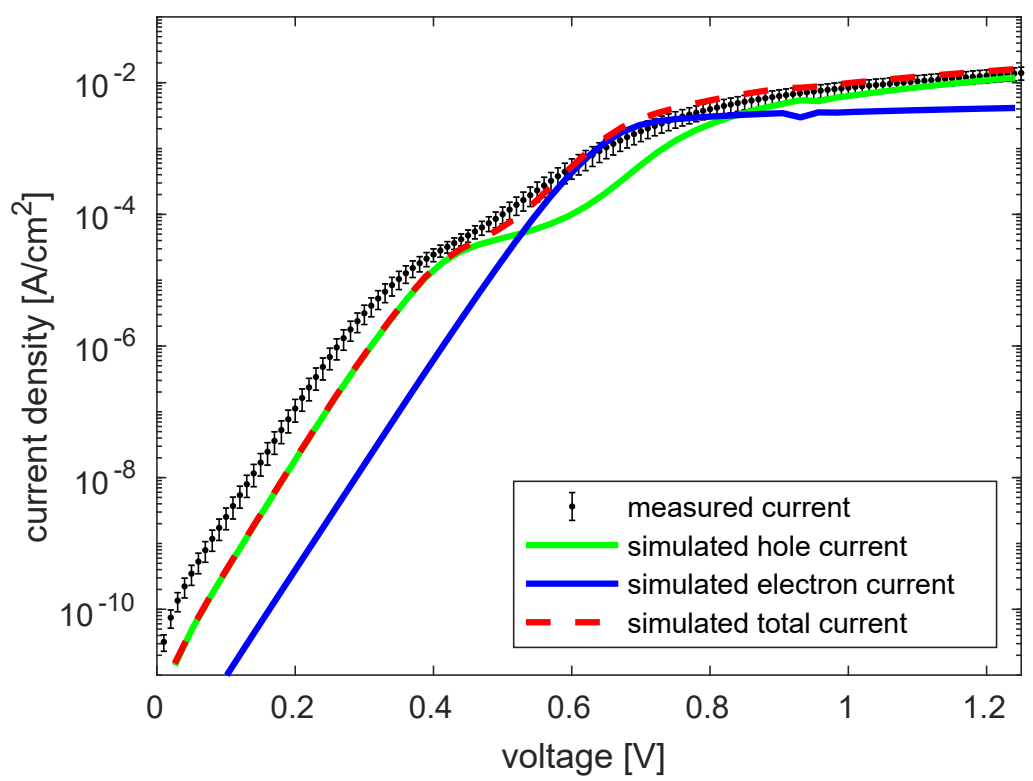

Figure 6.7: Simulated fit for the measured data of a $\mathrm{Au} / \mathrm{SiO}_{2} / \mathrm{Si} \mathrm{MIS}$ diode. The simulation parameters used are $N_{D}=6.71 \times 10^{14} \mathrm{~cm}^{-3}, \phi_{m}=4.8 \mathrm{eV}$, $t_{o x}=1.63 \mathrm{~nm}$, and electron and hole effective masses in oxide of $0.4 \mathrm{~m}_{e}$ and $0.3 \mathrm{~m}_{e}$ respectively. The distribution of surface states used in this simulation is shown in Figure 6.8.

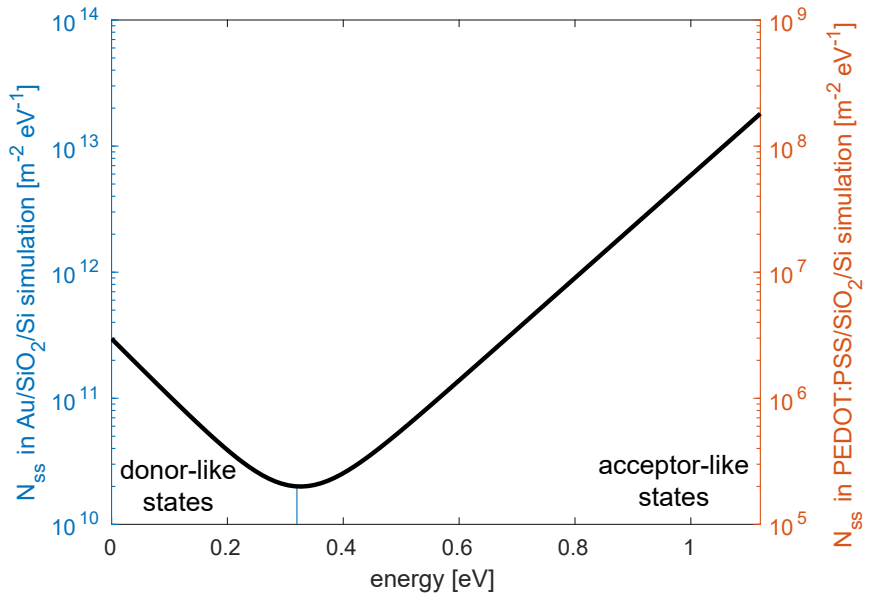

Figure 6.8: Interface state density at the $\mathrm{Si} / \mathrm{SiO}_{2}$ interface as defined by the DIGS model. The left-hand $\mathrm{y}$-axis corresponds to the surface state profile that gave the best fit for $\mathrm{Au} / \mathrm{SiO}_{2} / \mathrm{Si}$ diodes. The right-hand y-axis corresponds to the surface state profile that gave the best fit for PEDOT:PSS $/ \mathrm{SiO}_{2} / \mathrm{Si}$ diodes. 


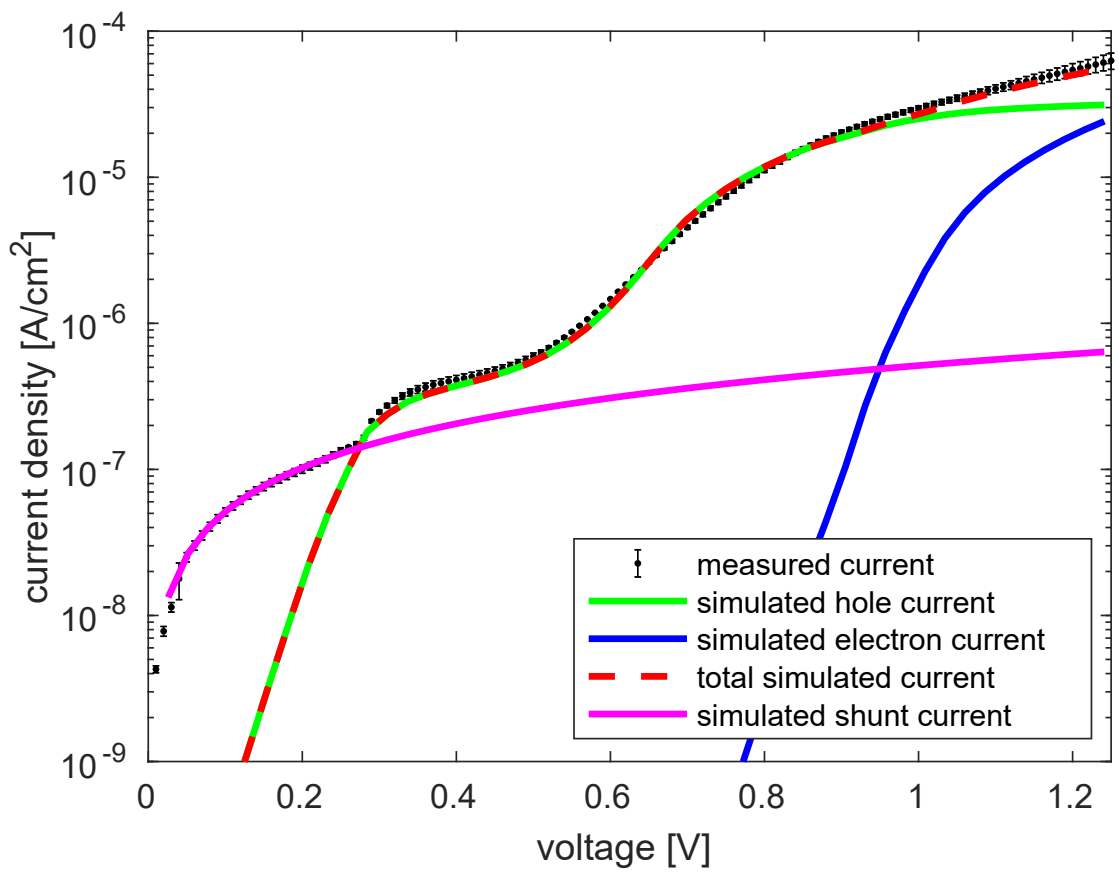

Figure 6.9: Measured and simulated current voltage characteristics of a typical PEDOT-based MIS diode. The simulation parameters used are $N_{D}=6.95 \times 10^{14} \mathrm{~cm}^{-3}, \phi_{m}=4.76 \mathrm{eV}, t_{o x}=1.93 \mathrm{~nm}$, and electron and hole effective masses in oxide of $0.4 \mathrm{~m}_{e}$ and $0.3 \mathrm{~m}_{e}$ respectively. The distribution of surface states used in this simulation is shown in Figure 6.8.

of $\mathrm{Au} / \mathrm{SiO}_{2} / \mathrm{Si}$ diodes, but the best fit to measured data was obtained for an oxide thickness of $t_{o x}=1.93 \mathrm{~nm}$. This apparent increase in the "oxide thickness" is consistent with accumulation of non-conductive PSS at the surface as proposed by Koch [132]. The interface state density required for the best fit was also lower then for the $\mathrm{Au} / \mathrm{SiO}_{2} / \mathrm{Si}$ diodes. The distribution of surface states used in this simulation is shown in Figure 6.8. This is consistent with a better surface passivation provided by a thicker tunneling barrier. Additionally, it suggests that the $\mathrm{Si} / \mathrm{SiO}_{2}$ interface is getting contaminated with $\mathrm{Au}$ in the inorganic diodes, where as no such degradation occurs in the hybrid devices.

The high current density at voltages below $0.25 \mathrm{~V}$ is due to a parasitic shunt resistance, determined from the current-voltage characteristic to be approximately $2 \mathrm{M} \Omega$. The $\mathrm{Au} / \mathrm{SiO}_{2} / \mathrm{Si}$ diodes displayed a similar shunt characteristic at lower biases 
when probed with a sharp-tip probe. However, when a dull, spring-loaded probe was used, the shunt disappeared, as can be seen in the measured data presented here. This was not the case for the PEDOT:PSS/SiO $/$ /Si, likely due to PEDOT:PSS being much softer than Au. The shunt resistance appeared to decrease with increasing probe pressure for both types of diodes. These observations indicate that the parasitic shunt may be associated with pressure induced bandgap narrowing, well documented in cases of four-point probe and spreading resistance measurements. [138-141]

The simulations, results of which are shown in Figure 6.9, indicate that the current-voltage characteristic of the PEDOT-based diode below a bias of $1 \mathrm{~V}$ can be accounted for entirely with only the hole current component. At higher biases, the electron current starts to contribute to the IV characteristic. The simulation parameters used are $N_{D}=6.95 \times 10^{14} \mathrm{~cm}^{-3}, \phi_{m}=4.76 \mathrm{eV}$, and $t_{o x}=1.93 \mathrm{~nm}$.

The shape of the simulated current-voltage characteristic is due to the alignment of the HOMO band in PEDOT:PSS to the valence band of the silicon substrate. The current between the LUMO and the conduction bands does not contribute to the overall IV characteristic until the bias exceeds $1 \mathrm{~V}$ due to the low probability of occupation in the conduction band away from the bandgap. Figure 6.10 shows the simulated band distribution in PEDOT:PSS at $0 \mathrm{~V}$ bias. Both the HOMO and the LUMO bands were simulated to be $0.6 \mathrm{eV}$ to coincide with the absorption measurements shown in Figure 6.6(a). The bipolaron bands were simulated to be $0.2 \mathrm{eV}$ thick. For a fit to measured data the lower bipolaron band had to be well above the Fermi level of PEDOT. This energy arrangement makes the bipolaron band holefilled, which is consistent with theory $[61,62]$. The position of the HOMO band below the Fermi level determines the ratio between the hole current in depletion and in accumulation. As such, it was adjusted to best match the measured current. 


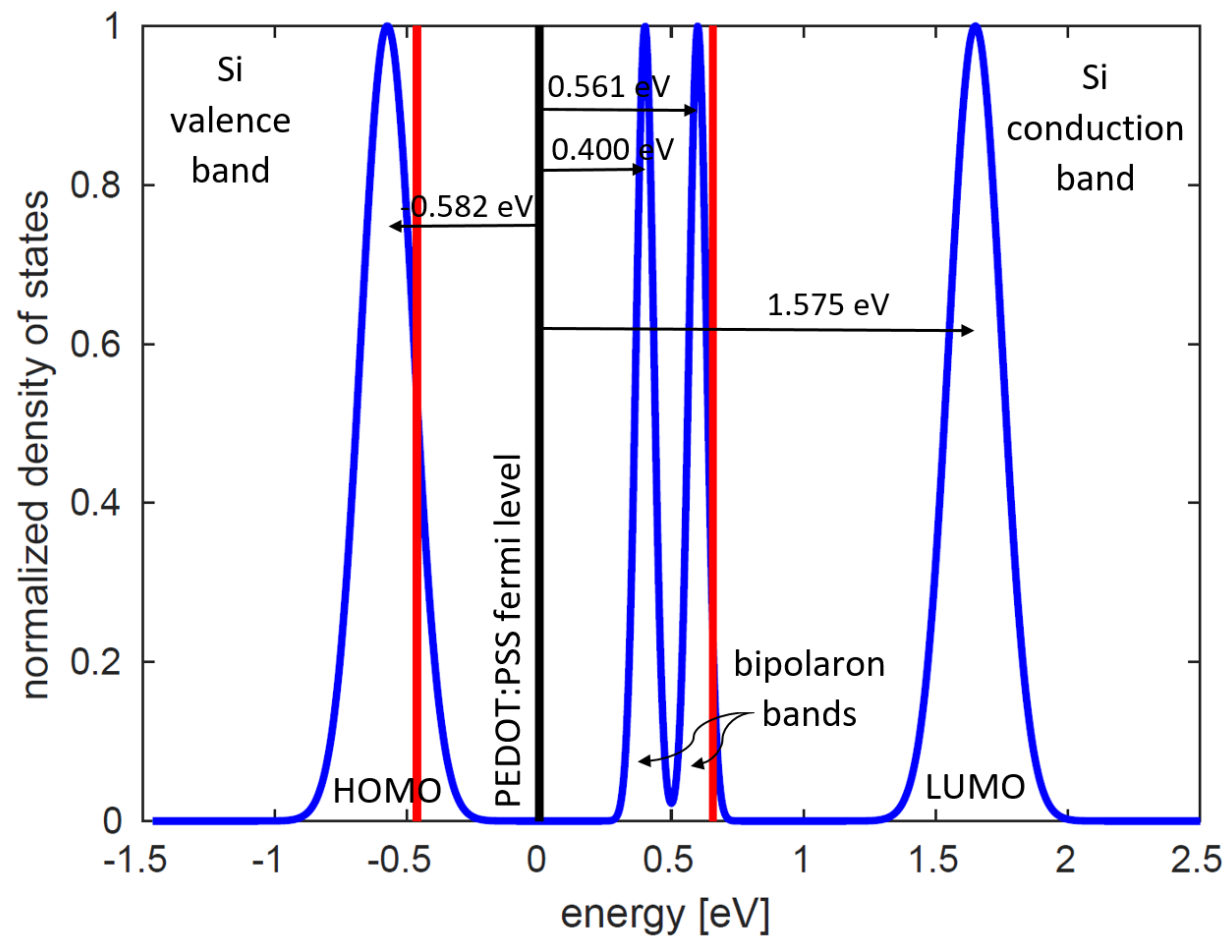

Figure 6.10: Simulated PEDOT:PSS bands at $0 \mathrm{~V}$ applied bias. The density of states is adjusted such that the carrier density of $N_{\text {holes }}=3 \times 10^{20} \mathrm{~cm}^{-3}$. The red lines indicate the edges of the conduction and valence bands of the silicon substrate. The black line indicates the position of the Fermi level. 


\subsection{Conclusion}

The simulations performed in this work confirm that the work functions of gold and PEDOT:PSS are very similar. Both materials used as a top conductor in an MIS device are capable of inverting the surface and producing minority carrier diodes. However, the relatively thin energy bands of PEDOT:PSS considerably limit the current density in hybrid devices. Additionally, the accumulation of non-conductive PSS at the interface with $\mathrm{SiO}_{2}$ effectively increases the tunneling barrier, reducing the current density further. As such, a PEDOT:PSS model should include a tunneling barrier, as well as a limited energy range on the order of $0.6 \mathrm{eV}$ over which the charge transitions in and out of the polymer are allowed. In the course of the model development, it became evident that the shape of the density of states over the HOMO band does not influence the resultant current density. A rectangular state density profile should be sufficient and easiest to implement.

According to the theory of organic conductors, the width of the energy bands is a function of interaction between the polymer chains [61]. Future research to examine how the degree of polymer alignment affects the HOMO band width would be relevant. Theoretically, if a better polymer alignment is achieved, higher current densities should be possible.

The accumulation of PSS at the substrate-polymer interface could be used to an advantage if the process can be controlled. If the silicon surface can be prepared to encourage PSS accumulation, it's possible dielectric growth or deposition might not be necessary for MIS diode formation, with the PSS comprising the entirety of the tunneling barrier. If feasible, this fabrication approach would simplify device processing and decrease the energy budget required. 


\section{Chapter 7}

\section{Conclusions}

This thesis has advanced the understanding of the electronic structure of conductive polymers and its effects on hybrid devices through the analysis of silicon and poly $(3,4-$ ethylenedioxythiophene) (PEDOT) interaction in a metal-insulator-semiconductor diode architecture for photovoltaic applications.

A proof-of-concept experiment demonstrated that a hybrid organic metalinsulator-semiconductor diode with PEDOT:PSS can operate as a minority carrier diode and function as a photovoltaic device. The test devices were constructed on flat $n$-type silicon substrates with thermally grown $\mathrm{SiO}_{2}$ as the insulator and commercially available high conductivity PEDOT:PSS. The best efficiency achieved over the course of this experiment for non-optimized devices was 1.65\%. For optimized devices, the efficiency was predicted to be around $12 \%$ [71].

A variety of PEDOT formulations were characterized and assessed for use in the hybrid MIS photovoltaic devices. A composite of in situ polymerized PEDOT and sol-gel silica was found to be the most attractive candidate. It was found to exhibit a high work function of approximately $5 \mathrm{eV}$, high enough to force the diode into minority carrier-operation, showed promising conductivity up to $430 \mathrm{~S} / \mathrm{cm}$, excellent transparency, and capability to fill high aspect ratio nanostructures.

It was also established that nanostructuring the silicon substrate surface is a viable 
way to increase the efficiency of hybrid MIS devices despite challenges presented by the increased device complexity. Surface structuring effectively increases the pathlength of the light, allowing more of it to be absorbed in the substrate, thereby increasing device efficiency. To maximize the effectiveness of this technique, the nanostructure height should exceed the light wavelength and the feature size should be smaller than the wavelength of interest. For effective trapping of light in the optical range of $300 \mathrm{~nm}$ to $1000 \mathrm{~nm}$, high aspect ratio nanostructures are required. Two potential pitfalls associated with such nanostructuring were identified in this work: the effective increase in the surface trap density and the light attenuation by the bulk of the polymer, filling the structure. Analysis shows that the polymer attenuation can be effectively mitigated by increasing PEDOT transparency at the cost of lowering conductivity without substantially compromising device efficiency. As for surface traps, better surface passivation would improve solar cell performance, however, experimental evidence shows that even at passivation levels achieved in this work, the benefit of nanostructuring more than outweighs associated increased losses, with a resultant five-fold increase in efficiency.

The electrical characteristics of the hybrid MIS diodes as compared with their conventional inorganic counterparts, constructed with gold metal reveal that the current density in the hybrid devices is limited by the span of the thin energy bands in the polymeric conductor. This fundamental feature of organic conductors, normally overlooked in the literature, may prove to be a limiting factor for hybrid photovoltaic devices. 


\subsection{Future Work}

Better surface passivation would undoubtedly improve device performance. The challenge, however, is achieving it with dielectric thicknesses on the order of a few nanometers, with good thickness control and repeatability. Recently, surface passivation improvements for ALD alumina have been reported [40-42]. Examination of silicon surface passivation capabilities of various ALD dielectrics, especially ones with lower electron affinities, should be conducted so that thicker dielectrics could be used in MIS architectures without compromising tunneling efficiency.

Theoretically, additional benefits may be extracted from surface structuring, if it can be made fine enough. Creating surface structures with feature sizes on the order of a few nanometers should lead to widening of the bandgap of the substrate [142]. Such techniques can theoretically be used to adjust the silicon bandgap to better match the solar spectrum. An additional benefit of nanostructuring with such fine feature sizes is a possibility of a pseudo-direct behaviour of the bandgap in silicon. As the spatial confinement is increased, so is the uncertainty in momentum, allowing for direct recombination [143]. Such a shift in the recombination behaviour might help close the gap in efficiencies between the $\mathrm{Si}$ and direct-bandgap-material solar cells, such as GaAs. The glancing angle deposited (GLAD) silicon nanotubes, pioneered by Brett, have silicon structures approaching the dimensions needed to affect the bandgap [118] and should be studied for potential use in hybrid solar cells.

Since the width of the energy bands in polymeric conductors limits the current density, it is necessary, for the future progress of hybrid MIS solar cells, to determine a method to widen them. According to the theory of organic conductors, the width of the energy bands is a function of interaction between the polymer chains [61]. A number of methods have been proposed in the literature to achieve better chain alignment, mostly in the efforts to increase conductivity $[99,100,104]$. It would be interesting to 
examine how the degree of polymer alignment affects the HOMO band width. Theoretically, if a better polymer alignment is achieved, higher current densities should be possible.

It has been noted in this work that a layer of non-conductive PSS tends to accumulate at the silicon PEDOT:PSS interface. If this process could be controlled, then it should be possible to fabricate an MIS diode without dielectric growth or deposition with the PSS comprising the entirety of the tunneling barrier. If feasible, this fabrication approach would simplify device processing and decrease the energy budget required. As such, silicon surface preparation techniques that could encourage PSS accumulation should be examined. The surface passivation properties of PSS would also have to be studied.

\subsection{Disseminations}

The proof-of-concept experiment for organic hybrid MIS photovoltaic diodes was presented at the 2010 Photovoltaics Canada Conference. An accompanying paper was then published in the conference proceedings [71]. This work reports on the test results of the fabricated photovoltaic devices and their potential for photovoltaic applications. It is the result of a collaborative interdisciplinary project at Carleton University between the Departments of Electronics, providing silicon processing and materials/device testing expertise, and Department of Chemistry providing surface chemistry expertise required for atomic layer deposition as a part of the fabrications process for a portion of the project. This work continued and inspired two other

presentations at the Photovoltaics Canada Conference (2011) [144] and the $12^{\text {th }}$ International Conference on Atomic Layer Deposition (2012) [145].

The development of the gold-assisted chemical etch and the dry reactive ion etch processes as well as the experimental assessment of surface nanostructuring for solar 
cell efficiency optimization was presented as a poster at Materials Research Society 2010 Spring Meeting [146].

The preliminary results of comparison between the hybrid and conventional MIS devices using an early version of the simulator were presented at the Materials Research Society 2013 Fall Meeting. The results indicated that the width of the energy bands would play a large role in the device behaviour [147]. The work continued and a comprehensive report on the findings of this comparative work was compiled into a paper to be submitted to the IEEE Transactions on Electron Devices [148]. 


\section{List of References}

[1] Ren21:Renewable Energy Policy Network for the 21st century. Renewables 2016: Global status report. [Online]. Available: http://www.ren21.net/ wp-content/uploads/2016/06/GSR_2016_KeyFindings1.pdf

[2] International Energy Agency. Key World Energy Statistics. [Online]. Available: https://www.iea.org/publications/freepublications/publication/ KeyWorld2016.pdf

[3] M. Wright and A. Uddin, "Organic-inorganic hybrid solar cells: A comparative review," Solar energy materials and solar cells, vol. 107, pp. 87-111, 2012.

[4] C.-F. Lin, W.-F. Su, C.-I. Wu, and I.-C. Cheng, Organic, Inorganic and Hybrid Solar Cells: Principles and Practice. New Jersey: John Wiley \& Sons, 2012.

[5] J. Nelson, The Physics of Solar Cells. Imperial College press London, 2003.

[6] S. R. Wenham, M. A. Green, M. E. Watt, and R. Corkish, Applied Photovoltaics. Earthscan, 2007.

[7] C. Honsberg and S. Bowden. PVCDROM: Standard Solar Spectra. [Online]. Available: http://pveducation.org/pvcdrom/appendices/ standard-solar-spectra

[8] S. M. Sze and K. K. Ng, Physics of Semiconductor Devices. John Wiley \& Sons, 2006.

[9] C. Honsberg and S. Bowden. PVCDROM. [Online]. Available: http: //pveducation.org/

[10] Manitoba Hydro. Utility rate comparisons. [Online]. Available: https://www.hydro.mb.ca/regulatory_affairs/energy_rates/electricity/ utility_rate_comp.shtml 
[11] A. Morrow and T. Cardoso, "ONTARIO POWER PLAY; Ontarians pay steeper rates for their power than any other province as a result of a decade's worth of policy choices. Adrian Morrow and Tom Cardoso address the key questions about how we got here and what the province could do to fix it." Globe $\&$ Mail [Toronto, Canada], p. A8, 9 Jan. 2017.

[12] M. A. Green, K. Emery, Y. Hishikawa, W. Warta, and E. D. Dunlop, "Solar cell efficiency tables (Version 43)," Progress in Photovoltaics: Research and Applications, vol. 22, no. 1, pp. 1-9, 2014, PIP-13-263.

[13] M. A. Green, "Consolidation of thin-film photovoltaic technology: The coming decade of opportunity," Progress in Photovoltaics, vol. 14, no. 5, pp. 383-392, 2006.

[14] M. A. Green, K. Emery, D. L. King, Y. Hishikawa, and W. Warta, "Solar cell efficiency tables (Version 28)," Progress in Photovoltaics: Research and Applications, vol. 14, no. 5, pp. 455-461, 2006.

[15] M. A. Green, K. Emery, Y. Hishikawa, W. Warta, and E. D. Dunlop, "Solar cell efficiency tables (Version 48)," Progress in Photovoltaics: Research and Applications, vol. 24, no. 7, pp. 905-913, 2016.

[16] D. L. Pulfrey, "MIS solar cells: A review," IEEE Transactions on Electron Devices, vol. 25, no. 11, pp. 1308-1317, Nov 1978.

[17] V. Temple, M. Green, and J. Shewchun, "Equilibrium-to-nonequilibrium transition in MOS (surface oxide) tunnel diode," Journal of Applied Physics, vol. 45, no. 11, pp. 4934-4943, 1974.

[18] M. Green, F. King, and J. Shewchun, "Minority carrier MIS tunnel diodes and their application to electron-and photo-voltaic energy conversionI. Theory," Solid-State Electronics, vol. 17, no. 6, pp. 551-561, 1974.

[19] N. G. Tarr, D. L. Pulfrey, and D. S. Camporese, "An analytic model for the MIS tunnel junction," IEEE Transactions on Electron Devices, vol. 30, no. 12, pp. 1760-1770, Dec 1983.

[20] S. Ashok, P. P. Sharma, and S. J. Fonash, "Spray-deposited ITO-Silicon SIS heterojunction solar cells," IEEE Transactions on Electron Devices, vol. 27, no. 4, pp. 725-730, Apr 1980. 
[21] H. Cachet, J. Bruneaux, G. Folcher, C. Levy-Clement, C. Vard, and M. Neumann-Spallart, "n-Si/SnO 2 junctions based on macroporous silicon for photoconversion," Solar Energy Materials and Solar Cells, vol. 46, no. 2, pp. 101-114, 1997.

[22] Q. Wei, H. Zheng, and Y. Huang, "Direct patterning ITO transparent conductive coatings," Solar Energy Materials and Solar Cells, vol. 68, no. 3, pp. 383-390, 2001.

[23] J. Ederth, P. Heszler, A. Hultåker, G. Niklasson, and C. Granqvist, "Indium tin oxide films made from nanoparticles: Models for the optical and electrical properties," Thin Solid Films, vol. 445, no. 2, pp. 199-206, 2003.

[24] C. G. Granqvist and A. Hultåker, "Transparent and conducting ITO films: New developments and applications," Thin Solid Films, vol. 411, no. 1, pp. 1-5, 2002.

[25] A. K. Ghosh, C. Fishman, and T. Feng, "Theoretical efficiency of $\mathrm{SnO}_{2} / \mathrm{Si}$ solar cells," Journal of Applied Physics, vol. 50, no. 5, pp. 3454-3458, 1979.

[26] D. M. Mattox and V. H. Mattox, "Review of Transparent Conductive Oxides (TCO)."

[27] B. G. Lewis and D. C. Paine, "Applications and processing of transparent conducting oxides," MRS Bulletin, vol. 25, no. 08, pp. 22-27, 2000.

[28] A. Stadler, "Transparent conducting oxides - An up-to-date overview," Materials, vol. 5, no. 4, pp. 661-683, 2012.

[29] S. C. Baker-Finch and K. R. McIntosh, "Reflection distributions of textured monocrystalline silicon: implications for silicon solar cells," Progress in Photovoltaics: Research and Applications, vol. 21, no. 5, pp. 960-971, 2013.

[30] S.-Y. Lien, C.-H. Yang, C.-H. Hsu, Y.-S. Lin, C.-C. Wang, and D.-S. Wuu, "Optimization of textured structure on crystalline silicon wafer for heterojunction solar cell," Materials Chemistry and Physics, vol. 133, no. 1, pp. 63-68, 2012.

[31] R. Barrio, N. González, J. Cárabe, and J. J. Gandía, "Optimisation of NaOH texturisation process of silicon wafers for heterojunction solar-cells applications," Solar Energy, vol. 86, no. 3, pp. 845-854, 2012.

[32] R. B. Stephens and G. D. Cody, "Optical reflectance and transmission of a textured surface," Thin Solid Films, vol. 45, no. 1, pp. 19-29, 1977. 
[33] H. Kikuta, H. Yoshida, and K. Iwata, "Ability and limitation of effective medium theory for subwavelength gratings," Optical Review, vol. 2, no. 2, pp. 92-99, 1995.

[34] D. H. Raguin and G. M. Morris, "Antireflection structured surfaces for the infrared spectral region," Appl. Opt., vol. 32, no. 7, pp. 1154-1167, Mar 1993.

[35] X. Jing, J. Ma, S. Liu, Y. Jin, H. He, J. Shao, and Z. Fan, "Analysis and design of transmittance for an antireflective surface microstructure," Optics Express, vol. 17, no. 18, pp. 16119-16134, 2009.

[36] L. E. Black, Surface Recombination Theory. Springer, 2016.

[37] H. Hasegawa, T. Sato, S. Kasai, B. Adamowicz, and T. Hashizume, "Dynamics and control of recombination process at semiconductor surfaces, interfaces and nano-structures," Solar Energy, vol. 80, no. 6, pp. 629-644, 2006.

[38] H. Mackel and A. Cuevas, "Determination of the surface recombination velocity of unpassivated silicon from spectral photoconductance measurements," in Proceedings of 3rd World Conference on Photovoltaic Energy Conversion, 2003., vol. 1, May 2003, pp. 71-74 Vol.1.

[39] A. G. Aberle, S. Glunz, and W. Warta, "Impact of illumination level and oxide parameters on Shockley-Read-Hall recombination at the $\mathrm{Si}_{-} \mathrm{SiO}_{2}$ interface," Journal of Applied Physics, vol. 71, no. 9, pp. 4422-4431, 1992.

[40] G. Dingemans and W. Kessels, "Status and prospects of $\mathrm{Al}_{2} \mathrm{O}_{3}$-based surface passivation schemes for silicon solar cells," Journal of Vacuum Science \&f Technology A, vol. 30, no. 4, p. 040802, 2012.

[41] G. Dingemans, M. Van de Sanden, and W. Kessels, "Influence of the deposition temperature on the c-Si surface passivation by $\mathrm{Al}_{2} \mathrm{O}_{3}$ films synthesized by ALD and PECVD," Electrochemical and Solid-state Letters, vol. 13, no. 3, pp. H76H79, 2010.

[42] J. Schmidt, A. Merkle, R. Brendel, B. Hoex, M. Van de Sanden, and W. Kessels, "Surface passivation of high-efficiency silicon solar cells by atomiclayer-deposited $\mathrm{Al}_{2} \mathrm{O}_{3}$," Progress in Photovoltaics: Research and Applications, vol. 16, no. 6, pp. 461-466, 2008.

[43] N. Swami, S. Srivastava, and H. Ghule, "The role of the interfacial layer in Schottky barrier solar cells," Journal of Physics D: Applied Physics, vol. 12, no. 5, p. 765, 1979. 
[44] M. Morita, T. Ohmi, E. Hasegawa, M. Kawakami, and M. Ohwada, "Growth of native oxide on a silicon surface," Journal of Applied Physics, vol. 68, no. 3, pp. 1272-1281, 1990.

[45] E. Charlson and J. Lien, "An Al p-silicon MOS photovoltaic cell," Journal of Applied Physics, vol. 46, no. 9, pp. 3982-3987, 1975.

[46] R. Stirn and Y.-C. M. Yeh, "A 15\% efficient antireflection-coated metal-oxidesemiconductor solar cell," Applied Physics Letters, vol. 27, no. 2, pp. 95-98, 1975 .

[47] W. Henrion, M. Rebien, H. Angermann, and A. Röseler, "Spectroscopic investigations of hydrogen termination, oxide coverage, roughness, and surface state density of silicon during native oxidation in air," Applied Surface Science, vol. 202, no. 3, pp. 199-205, 2002.

[48] R. Singh, "Growth of thin thermal silicon dioxide films with low defect density," Microelectronics Journal, vol. 23, no. 4, pp. 273-281, 1992.

[49] M. Green, E. Gusev, R. Degraeve, and E. Garfunkel, "Ultrathin (< $4 \mathrm{~nm}) \mathrm{SiO}_{2}$ and $\mathrm{Si}-\mathrm{O}-\mathrm{N}$ gate dielectric layers for silicon microelectronics: Understanding the processing, structure, and physical and electrical limits," Journal of Applied Physics, vol. 90, no. 5, pp. 2057-2121, 2001.

[50] M. Uematsu, H. Kageshima, and K. Shiraishi, "Simulation of wet oxidation of silicon based on the interfacial silicon emission model and comparison with dry oxidation," Journal of Applied Physics, vol. 89, no. 3, pp. 1948-1953, 2001.

[51] M. Naito, N. Momma et al., "A practical model for growth kinetics of thermal $\mathrm{SiO}_{2}$ on silicon applicable to a wide range of oxide thickness," Solid-State Electronics, vol. 29, no. 9, pp. 885-891, 1986.

[52] K. Kim, Y. H. Lee, M. H. An, M. S. Suh, C. J. Youn, K. B. Lee, and H. J. Lee, "Growth law of silicon oxides by dry oxidation," Semiconductor Science and Technology, vol. 11, no. 7, p. 1059, 1996.

[53] T. Zeng, H. Doumanidis, J. Hebb, and D. Brown, "Growth of ultrathin silicon dioxide films during rapid-thermal oxidation," in Advanced Thermal Processing of Semiconductors 9th Internationa Conference on RTP 2001. IEEE, 2001, pp. 287-295. 
[54] D. O. Kuznetsov, "Silicon thermal oxidation models comparison used in TCAD Sentaurus process and fact," in 2008 9th International Workshop and Tutorials on Electron Devices and Materials, 2008.

[55] M. Offenberg, M. Liehr, G. Rubloff, and K. Holloway, "Ultraclean, integrated processing of thermal oxide structures," Applied Physics Letters, vol. 57, no. 12, pp. 1254-1256, 1990.

[56] V. K. Bhat, M. Pattabiraman, K. Bhat, and A. Subrahmanyam, "The growth of ultrathin oxides of silicon by low temperature wet oxidation technique," $M a$ terials Research Bulletin, vol. 34, no. 10, pp. 1797-1803, 1999.

[57] A. L. Brazeau and S. T. Barry, "Atomic layer deposition of aluminum oxide thin films from a heteroleptic, amidinate-containing precursor," Chemistry of Materials, vol. 20, no. 23, pp. 7287-7291, 2008.

[58] Y. Zhang, J. A. Bertrand, R. Yang, S. M. George, and Y. Lee, "Electroplating to visualize defects in $\mathrm{Al}_{2} \mathrm{O}_{3}$ thin films grown using atomic layer deposition," Thin Solid Films, vol. 517, no. 11, pp. 3269-3272, 2009.

[59] K. Gao, F. Speck, K. Emtsev, T. Seyller, L. Ley, M. Oswald, and W. Hansch, "Interface of atomic layer deposited $\mathrm{Al}_{2} \mathrm{O}_{3}$ on H-terminated silicon," Physica Status Solidi (A), vol. 203, no. 9, pp. 2194-2199, 2006.

[60] T. Kääriäinen, D. Cameron, M.-L. Kääriäinen, and A. Sherman, Atomic layer deposition: principles, characteristics, and nanotechnology applications. John Wiley \& Sons, 2013.

[61] W. Brütting and C. Adachi, Physics of Organic Semiconductors, Second Edition. John Wiley \& Sons, 2012.

[62] A. Elschner, S. Kirchmeyer, W. Lovenich, U. Merker, and K. Reuter, PEDOT: Principles and Applications of an Intrinsically Conductive Polymer. New York: CRC Press, 2010.

[63] N. S. Sariciftci and S.-S. Sun, Organic Photovoltaics: Mechanism, Materials, and Devices. New York: Taylor \& Francis, 2005.

[64] A. Erickson, A. Zohar, and D. Cahen, "Inversion layers in PEDOT:PSS - n-Si hybrid junction," in MRS 2013 Spring Meeting, 2013. 
[65] Heraeus Deutschland GmbH \& Co. KG. Conductive Polymers. [Online]. Available: http://www.heraeus-clevios.com/en/conductivepolymers/ pedot-pss-conductive-polymers.aspx

[66] Heraeus Deutschland GmbH, "ITO Alternative: solution deposited Clevios PEDOT:PSS for transparent conductive applications," in Trade Article, 2012.

[67] N. Koch, A. Elschner, J. P. Rabe, and R. L. Johnson, "Work Function Independent Hole-Injection Barriers Between Pentacene and Conducting Polymers," Advanced Materials, vol. 17, no. 3, pp. 330-335, 2005.

[68] G. Greczynski, T. Kugler, and W. R. Salaneck, "Energy level alignment in organic-based three-layer structures studied by photoelectron spectroscopy," Journal of Applied Physics, vol. 88, no. 12, pp. 7187-7191, 2000.

[69] Y. Lee and J. Kim, "Transparent and conductive composite of poly (3, 4ethylenedioxythiophene) and silica sol-gel materials," Molecular Crystals and Liquid Crystals, vol. 337, no. 1, pp. 213-216, 1999.

[70] Y. S. Kim, S. B. Oh, J. H. Park, M. S. Cho, and Y. Lee, "Highly conductive PEDOT/silicate hybrid anode for ITO-free polymer solar cells," Solar Energy Materials and Solar Cells, vol. 94, no. 3, pp. 471-477, 2010.

[71] S. Demtchenko, S. McGarry, P. Gordon, S. Barry, and N. G. Tarr, "Characterization and assessment of a novel hybrid organic/inorganic metal-insulatorsemiconductor structure for photovoltaic applications," in Photonics North 2010. International Society for Optics and Photonics, 2010, pp. 77502Y$77502 \mathrm{Y}$.

[72] J. Zhang, Y. Zhang, F. Zhang, and B. Sun, "Electrical characterization of inorganic-organic hybrid photovoltaic devices based on silicon-poly (3, 4ethylenedioxythiophene): poly (styrenesulfonate)," Applied Physics Letters, vol. 102, no. 1, p. 013501, 2013.

[73] T.-G. Chen, B.-Y. Huang, E.-C. Chen, P. Yu, and H.-F. Meng, "Micro-textured conductive polymer/silicon heterojunction photovoltaic devices with high efficiency," Applied Physics Letters, vol. 101, no. 3, p. 033301, 2012.

[74] D. Vasileska, S. Goodnick, and G. Klimeck, Computational Electronics: Semiclassical and Quantum Device Modeling and Simulation. New York: CRC Press, 2010, the Drift-Diffusion Equations and Their Numerical Solution. 
[75] J. C. Ranuárez, M. J. Deen, and C.-H. Chen, "A review of gate tunneling current in MOS devices," Microelectronics reliability, vol. 46, no. 12, pp. 19391956, 2006.

[76] J. Shewchun and V. Temple, "Theoretical tunneling current characteristics of the SIS (semiconductor-insulator-semiconductor) diode," Journal of Applied Physics, vol. 43, no. 12, pp. 5051-5061, 1972.

[77] J. Shewchun, A. Waxman, and G. Warfield, "Tunneling in MIS structures - I: Theory," Solid-State Electronics, vol. 10, no. 12, pp. 1165-1186, 1967.

[78] T. Yeh, Q. Zhu, D. Buchholz, A. Martinson, R. P. Chang, and T. O. Mason, "Amorphous transparent conducting oxides in context: Work function survey, trends, and facile modification," Applied Surface Science, vol. 330, pp. 405-410, 2015 .

[79] A. Klein, C. Körber, A. Wachau, F. Säuberlich, Y. Gassenbauer, S. P. Harvey, D. E. Proffit, and T. O. Mason, "Transparent conducting oxides for photovoltaics: manipulation of Fermi level, work function and energy band alignment," Materials, vol. 3, no. 11, pp. 4892-4914, 2010.

[80] Y. Zhou, C. Fuentes-Hernandez, J. Shim, J. Meyer, A. J. Giordano, H. Li, P. Winget, T. Papadopoulos, H. Cheun, J. Kim et al., "A universal method to produce low-work function electrodes for organic electronics," Science, vol. 336, no. 6079, pp. 327-332, 2012.

[81] H. Hosono, D. C. Paine, and D. Ginley, Handbook of Transparent Conductors. Springer Science \& Business Media, 2010.

[82] C. Arenas and G. Sánchez, "Optical, electrical and morphological properties of transparent binary doped polyaniline thin films synthesized by in situ chemical bath deposition," Polymer International, vol. 60, no. 7, pp. 1123-1128, 2011.

[83] Y. Cao, G. M. Treacy, P. Smith, and A. J. Heeger, "Solution-cast films of polyaniline: Optical-quality transparent electrodes," Applied Physics Letters, vol. 60, no. 22, pp. 2711-2713, 1992.

[84] J. Jang, J. Ha, and K. Kim, "Organic light-emitting diode with polyaniline-poly (styrene sulfonate) as a hole injection layer," Thin Solid Films, vol. 516, no. 10, pp. 3152-3156, 2008. 
[85] L. S. Priyadharshni and M. Selvaraj, "Polypyrrole Sulfonate as a Transparent Conducting Film for Photovoltaic Applications," International Journal of Polymeric Materials and Polymeric Biomaterials, vol. 64, no. 1, pp. 47-53, 2015.

[86] G. Qi, Z. Wu, and H. Wang, "Highly conductive and semitransparent freestanding polypyrrole films prepared by chemical interfacial polymerization," Journal of Materials Chemistry C, vol. 1, no. 42, pp. 7102-7110, 2013.

[87] P. S. Abthagir and R. Saraswathi, "Junction properties of metal/polypyrrole Schottky barriers," Journal of Applied Polymer Science, vol. 81, no. 9, pp. 2127-2135, 2001.

[88] O. Inganäs, T. Skotheim, and I. Lundström, "Polypyrrole-semiconductor Schottky barriers," Journal of Applied Physics, vol. 54, no. 6, pp. 3636-3639, 1983.

[89] J. K. Wassei and R. B. Kaner, "Graphene, a promising transparent conductor," Materials Today, vol. 13, no. 3, pp. 52-59, 2010.

[90] Y. Lin, X. Li, D. Xie, T. Feng, Y. Chen, R. Song, H. Tian, T. Ren, M. Zhong, $\mathrm{K}$. Wang et al., "Graphene/semiconductor heterojunction solar cells with modulated antireflection and graphene work function," Energy \&5 Environmental Science, vol. 6, no. 1, pp. 108-115, 2013.

[91] X. Li, Y. Zhu, W. Cai, M. Borysiak, B. Han, D. Chen, R. D. Piner, L. Colombo, and R. S. Ruoff, "Transfer of large-area graphene films for high-performance transparent conductive electrodes," Nano Letters, vol. 9, no. 12, pp. 4359-4363, 2009 .

[92] L. Gomez De Arco, Y. Zhang, C. W. Schlenker, K. Ryu, M. E. Thompson, and C. Zhou, "Continuous, highly flexible, and transparent graphene films by chemical vapor deposition for organic photovoltaics," ACS Nano, vol. 4, no. 5, pp. 2865-2873, 2010.

[93] J. Wu, H. A. Becerril, Z. Bao, Z. Liu, Y. Chen, and P. Peumans, "Organic solar cells with solution-processed graphene transparent electrodes," Applied Physics Letters, vol. 92, no. 26, p. 263302, 2008.

[94] X. Ma and H. Zhang, "Fabrication of graphene films with high transparent conducting characteristics," Nanoscale research letters, vol. 8, no. 1, pp. 1-6, 2013. 
[95] Y.-H. Ha, N. Nikolov, S. K. Pollack, J. Mastrangelo, B. D. Martin, and R. Shashidhar, "Towards a Transparent, Highly Conductive Poly (3, 4ethylenedioxythiophene)," Advanced Functional Materials, vol. 14, no. 6, pp. 615-622, 2004.

[96] J. S. Choi, J.-H. Yim, D.-W. Kim, J.-K. Jeon, Y. S. Ko, and Y. Kim, "Effects of various imidazole-based weak bases and surfactant on the conductivity and transparency of poly (3, 4-ethylenedioxythiophene) films," Synthetic Metals, vol. 159, no. 23, pp. 2506-2511, 2009.

[97] Heraeus Deutschland GmbH \& Co. KG. In-Situ Polymerisation. [Online]. Available: http://www.heraeus-clevios.com/en/technology/in-situ-polymerisation. asp

[98] Heraeus Deutschland GmbH and Co. KG. In-Situ Polymerisation. [Online]. Available: https://www.heraeus.com/en/group/products_and_solutions_ group/products_and_solutions_group_at_a_glance/products_and_solutions.aspx

[99] J. Rivnay, S. Inal, B. A. Collins, M. Sessolo, E. Stavrinidou, X. Strakosas, C. Tassone, D. M. Delongchamp, and G. G. Malliaras, "Structural control of mixed ionic and electronic transport in conducting polymers," Nature communications, vol. 7, 2016.

[100] S. Timpanaro, M. Kemerink, F. Touwslager, M. De Kok, and S. Schrader, "Morphology and conductivity of PEDOT/PSS films studied by scanning-tunneling microscopy," Chemical Physics Letters, vol. 394, no. 4, pp. 339-343, 2004.

[101] F. Keohan, "Technical Marketing Specialist. Electronics and Optics Group. H.C.Stark, Inc," Personal Communication, 2010.

[102] Y. Zhou, H. Cheun, S. Choi, W. J. Potscavage Jr, C. Fuentes-Hernandez, and B. Kippelen, "Indium tin oxide-free and metal-free semitransparent organic solar cells," Applied Physics Letters, vol. 97, no. 15, p. 153304, 2010.

[103] Z. Li, F. Qin, T. Liu, R. Ge, W. Meng, J. Tong, S. Xiong, and Y. Zhou, "Optical properties and conductivity of PEDOT: PSS films treated by polyethylenimine solution for organic solar cells," Organic Electronics, vol. 21, pp. 144-148, 2015.

[104] J. Ouyang, "Secondary doping" methods to significantly enhance the conductivity of PEDOT: PSS for its application as transparent electrode of optoelectronic devices," Displays, vol. 34, no. 5, pp. 423-436, 2013. 
[105] H. Card and E. Rhoderick, "Studies of tunnel MOS diodes II. Thermal equilibrium considerations," Journal of Physics D: Applied Physics, vol. 4, no. 10, p. $1602,1971$.

[106] M. Kim, Y. S. Lee, Y. C. Kim, M. S. Choi, and J. Y. Lee, "Flexible organic light-emitting diode with a conductive polymer electrode," Synthetic Metals, vol. 161, no. 21, pp. 2318-2322, 2011.

[107] S. G. Im, K. K. Gleason, and E. A. Olivetti, "Doping level and work function control in oxidative chemical vapor deposited poly (3, 4ethylenedioxythiophene)," Applied Physics Letters, vol. 90, no. 15, p. 152112, 2007.

[108] K. Xing, M. Fahlman, X. Chen, O. Inganäs, and W. Salaneck, "The electronic structure of poly (3, 4-ethylene-dioxythiophene): studied by XPS and UPS," Synthetic Metals, vol. 89, no. 3, pp. 161-165, 1997.

[109] K. Mittal, "Adhesion measurement of thin films," Active and Passive Electronic Components, vol. 3, no. 1, pp. 21-42, 1976.

[110] L. L. Hench and J. K. West, "The sol-gel process," Chemical Reviews, vol. 90, no. 1, pp. 33-72, 1990.

[111] J. Yoo, I. Parm, U. Gangopadhyay, K. Kim, S. Dhungel, D. Mangalaraj, and J. Yi, "Black silicon layer formation for application in solar cells," Solar energy materials and solar cells, vol. 90, no. 18, pp. 3085-3093, 2006.

[112] L. Ma, Y. Zhou, N. Jiang, X. Lu, J. Shao, W. Lu, J. Ge, X. Ding, and X. Hou, "Wide-band "black silicon" based on porous silicon," Applied physics letters, vol. 88, no. 17, p. 171907, 2006.

[113] S. Koynov, M. S. Brandt, and M. Stutzmann, "Black nonreflecting silicon surfaces for solar cells," Applied Physics Letters, vol. 88, no. 20, p. 203107, 2006.

[114] M. Kroll, T. Käsebier, M. Otto, R. Salzer, R. Wehrspohn, E.-B. Kley, A. Tünnermann, and T. Pertsch, "Optical modeling of needle like silicon surfaces produced by an ICP-RIE process," in SPIE Photonics Europe. International Society for Optics and Photonics, 2010, pp. 772 505-772 505.

[115] K. Imamura, F. C. Franco Jr, T. Matsumoto, and H. Kobayashi, "Ultra-low reflectivity polycrystalline silicon surfaces formed by surface structure chemical transfer method," Applied Physics Letters, vol. 103, no. 1, p. 013110, 2013. 
[116] G. V. Morozov, R. Maev, and G. Drake, "Multiple reflection method for electromagnetic waves in layered dielectric structures," Quantum Electronics, vol. 31, no. 9, pp. 767-773, 2001.

[117] C.-L. Cheng, C.-W. Liu, J.-T. Jeng, B.-T. Dai, and Y.-H. Lee, "Fabrication and characterizations of black hybrid silicon nanomaterials as light-trapping textures for silicon solar cells," Journal of the Electrochemical Society, vol. 156, no. 5, pp. H356-H360, 2009.

[118] Z. Huang, K. D. Harris, and M. J. Brett, "Morphology control of nanotube arrays," Advanced Materials, vol. 21, no. 29, pp. 2983-2987, 2009.

[119] J. Yoo, G. Yu, and J. Yi, "Black surface structures for crystalline silicon solar cells," Materials Science and Engineering: B, vol. 159, pp. 333-337, 2009.

[120] H. Jansen, M. de Boer, R. Legtenberg, and M. Elwenspoek, "The black silicon method: A universal method for determining the parameter setting of a fluorinebased reactive ion etcher in deep silicon trench etching with profile control," Journal of Micromechanics and Microengineering, vol. 5, no. 2, p. 115, 1995.

[121] H. Jansen, M. De Boer, H. Wensink, B. Kloeck, and M. Elwenspoek, "The black silicon method. VIII. A study of the performance of etching silicon using $\mathrm{SF}_{6} / \mathrm{O}_{2}$-based chemistry with cryogenical wafer cooling and a high density ICP source," Microelectronics Journal, vol. 32, no. 9, pp. 769-777, 2001.

[122] H. Jansen, M. de Boer, B. Otter, and M. Elwenspoek, "The black silicon method IV: The fabrication of three-dimensional structures in silicon with high aspect ratios for scanning probe microscopy and other applications," 1995.

[123] H. M. Branz, V. E. Yost, S. Ward, K. M. Jones, B. To, and P. Stradins, "Nanostructured black silicon and the optical reflectance of graded-density surfaces," Applied Physics Letters, vol. 94, no. 23, p. 231121, 2009.

[124] Y. Lee, J. Kim, and Y. Son, "The preparation of organic inorganic conducting polymer composite films using in situ sol-gel process," Polymer (Korea), vol. 23, no. 3, pp. 443-449, 1999.

[125] M. Fabretto, J.-P. Autere, D. Hoglinger, S. Field, and P. Murphy, "Vacuum vapour phase polymerised poly (3, 4-ethyelendioxythiophene) thin films for use in large-scale electrochromic devices," Thin Solid Films, vol. 519, no. 8, pp. 2544-2549, 2011. 
[126] R. Saive, M. Scherer, C. Mueller, D. Daume, J. Schinke, M. Kroeger, and W. Kowalsky, "Imaging the electric potential within organic solar cells," Advanced Functional Materials, vol. 23, no. 47, pp. 5854-5860, 2013.

[127] B. Y. Finck and B. J. Schwartz, "Understanding the origin of the S-curve in conjugated polymer/fullerene photovoltaics from drift-diffusion simulations," Applied Physics Letters, vol. 103, no. 5, p. 053306, 2013.

[128] W. M. Haynes, CRC handbook of chemistry and physics. CRC press, 2014.

[129] K. Ng and H. Card, "A comparison of majority-and minority-carrier silicon MIS solar cells," IEEE Transactions on Electron Devices, vol. 27, no. 4, pp. 716-724, 1980 .

[130] L. Freeman and W. Dahlke, "Theory of tunneling into interface states," SolidState Electronics, vol. 13, no. 11, pp. 1483-1503, 1970.

[131] B. Adamowicz and H. Hasegawa, "Computer analysis of surface recombination process at $\mathrm{Si}$ and compound semiconductor surfaces and behavior of surface recombination velocity," Japanese journal of applied physics, vol. 37, no. 3S, p. 1631, 1998.

[132] N. Koch, A. Elschner, and R. L. Johnson, "Green polyfluorene-conducting polymer interfaces: Energy level alignment and device performance," Journal of applied physics, vol. 100, no. 2, p. 024512, 2006.

[133] P. A. Anderson, "Work function of gold," Physical Review, vol. 115, no. 3, p. $553,1959$.

[134] W. Sachtler, G. Dorgelo, and A. Holscher, "The work function of gold," Surface Science, vol. 5, no. 2, pp. 221-229, 1966.

[135] R. Archer and M. Atalla, "Metals contacts on cleaved silicon surfaces," Annals of the New York Academy of Sciences, vol. 101, no. 3, pp. 697-708, 1963.

[136] B. Brar, G. Wilk, and A. Seabaugh, "Direct extraction of the electron tunneling effective mass in ultrathin $\mathrm{SiO}_{2}, "$ Applied physics letters, vol. 69, no. 18, pp. 2728-2730, 1996.

[137] W.-C. Lee and C. Hu, "Modeling CMOS tunneling currents through ultrathin gate oxide due to conduction-and valence-band electron and hole tunneling," IEEE Transactions on Electron Devices, vol. 48, no. 7, pp. 1366-1373, 2001. 
[138] D. K. Schroder, Semiconductor material and device characterization. John Wiley \& Sons, 2006.

[139] L. Tan, L. Tan, M. Leong, R. Mazur, and C. Ye, "Characterization of ultrashallow dopant profiles using spreading resistance profiling," Journal of Vacuum Science \& Technology B, vol. 20, no. 1, pp. 483-487, 2002.

[140] S. T. Dunham, N. Collins, and N. Jeng, "Improved analysis of spreading resistance measurements," Journal of Vacuum Science 85 Technology B, vol. 12, no. 1, pp. 283-289, 1994.

[141] T. Clarysse, P. De Wolf, H. Bender, and W. Vandervorst, "Recent insights into the physical modeling of the spreading resistance point contact," Journal of Vacuum Science \& Technology B, vol. 14, no. 1, pp. 358-368, 1996.

[142] S. Sahu and K. Nanda, "Nanostructure semiconductors: physics and applications," PROCEEDINGS-INDIAN NATIONAL SCIENCE ACADEMY PART $A$, vol. 67, no. 1, pp. 103-130, 2001.

[143] V. Singh, Y. Yu, Q.-C. Sun, B. Korgel, and P. Nagpal, "Pseudo-direct bandgap transitions in silicon nanocrystals: effects on optoelectronics and thermoelectrics," Nanoscale, vol. 6, no. 24, pp. 14643-14647, 2014.

[144] A. Amos-Binks, B. Nemeth, S. Demtchenko, S. McGarry, and N. Tarr, "RF Sputtered aSi:H Solar Cells with Conductive Polymer Emitters," Oral presentation at: Photovoltaics 2011.

[145] P. Gordon, S. Barry, S. Demtchenko, and S. McGarry, "Metal-InsulatorSemiconductor Photovoltaic Devices Incorporating Black Silicon," Poster session at: AVS-ALD 2012 / Baltic-ALD 2012: 12th International Conference on Atomic Layer Deposition.

[146] S. Demtchenko, P. Gordon, S. McGarry, and S. Barry, "Hybrid Organic/Inorganic Metal-Insulator-Semiconductor (MIS) Photovoltaic Device," Poster presentation at: Materials Research Society 2010 Spring Meeting.

[147] S. Demtchenko, N. Tarr, and S. McGarry, "Effects of non-idealities of the organic conductor on the electrical characteristics of PEDOT:PSS/ $\mathrm{SiO}_{2} / \mathrm{Si}$ Schottky junctions," Poster presentation at: Materials Research Society 2013 Fall Meeting. 
[148] S. Demtchenko and S. McGarry, "Effect of PEDOT Band Structure on Conductive Polymer-Insulator-Silicon Junctions," To be submitted to IEEE Transactions on Electron Devices.

[149] E. Hecht, Optics. Addison Weley, 2002.

[150] D. Stauffer and A. Aharony, Introduction to percolation theory. CRC press, 1994.

[151] F. Bruder and A. Elschner, "Optical Constants of PEDOT Films," H.C. Stark Inc. Presentation Slides.

[152] D. M. Schwarz. Fast and Robust Curve Intersections. [Online]. Available: https://www.mathworks.com/matlabcentral/fileexchange/ 11837-fast-and-robust-curve-intersections 


\section{Appendix A}

\section{Measurement of an MIS Diode and Work Function Extraction}

Capacitance-voltage measurement is a very effective assessment tool for characterization of diodes. It allows for the extraction of built-in potential, $\psi_{b i}$, of the diode junction and, consequently, the metal work function, in case of a Schottky or an MIS diode, as well as, substrate doping density or diode active area (if one of these quantities is known).

For a Schottky diode the voltage and depletion capacitance are related as follows [8]:

$$
\frac{1}{C_{D}^{2}}=\frac{2\left(\psi_{b i}-V-k T / q\right)}{q \epsilon_{S i} N_{D} A^{2}}
$$

In a case of an MIS diode, an oxide capacitance appears in series with the depletion capacitance. However, if the oxide is very thin, the oxide capacitance is negligible for two capacitors in series. Thus, the measured capacitance becomes [8]:

$$
\frac{1}{C_{\text {measured }}^{2}}=\left(\frac{1}{C_{D}}+\frac{1}{C_{o x}}\right)^{2} \approx \frac{2\left(\psi_{b i}-V-k T / q\right)}{q \epsilon_{S i} N_{D} A^{2}}
$$

If $1 / C^{2}$ is plotted over $\mathrm{V}$, the x-intercept, $V_{i n t}$ yields the built-in potential, $\psi_{b i}$, 
from which the conductor work function, $\phi_{m}$, can be extracted [8].

$$
\begin{array}{r}
V_{i n t}=\psi_{b i}-k T / q \\
\psi_{b i}=\phi_{m}-\chi_{S i}-\phi_{n}-\phi_{o x}
\end{array}
$$

Here $\chi_{S i}$ is the electron affinity of silicon; $\phi_{o x}$ is the potential drop across the oxide and can be ignored for oxide thicknesses on the order of several nanometers; the barrier lowering effect can be expected to be low and was ignored. The position of the Fermi level below the conduction band, $\phi_{n}$, can be calculated from the resistivity of the substrate [8]:

$$
\begin{array}{r}
\phi_{n}=\frac{k_{B} T}{q} \ln \frac{N_{C}}{N_{D}} \\
N_{D}=\frac{1}{q \mu_{n} \rho}
\end{array}
$$

If the area of the device is known, the substrate doping density can also be extracted from equation A.2. The doping density is related to the slope, $m$, of the $V v s$ $1 / C^{2}$ characteristic as shown in equation A.5.

$$
N_{D}=\frac{2}{q \epsilon_{S i} A^{2} m}
$$

\section{A.1 Work Function Extraction for Diodes with PEDOT:PSS}

Several devices with varying oxide thicknesses were made with PEDOT:PSS PH750 as the top conductor. A reference device without any oxide was processed in parallel. The Figure A.1 shows the measured CV characteristic of these devices. The characteristics are wonderfully linear, indicating depletion diode and justifying the analysis described above. 


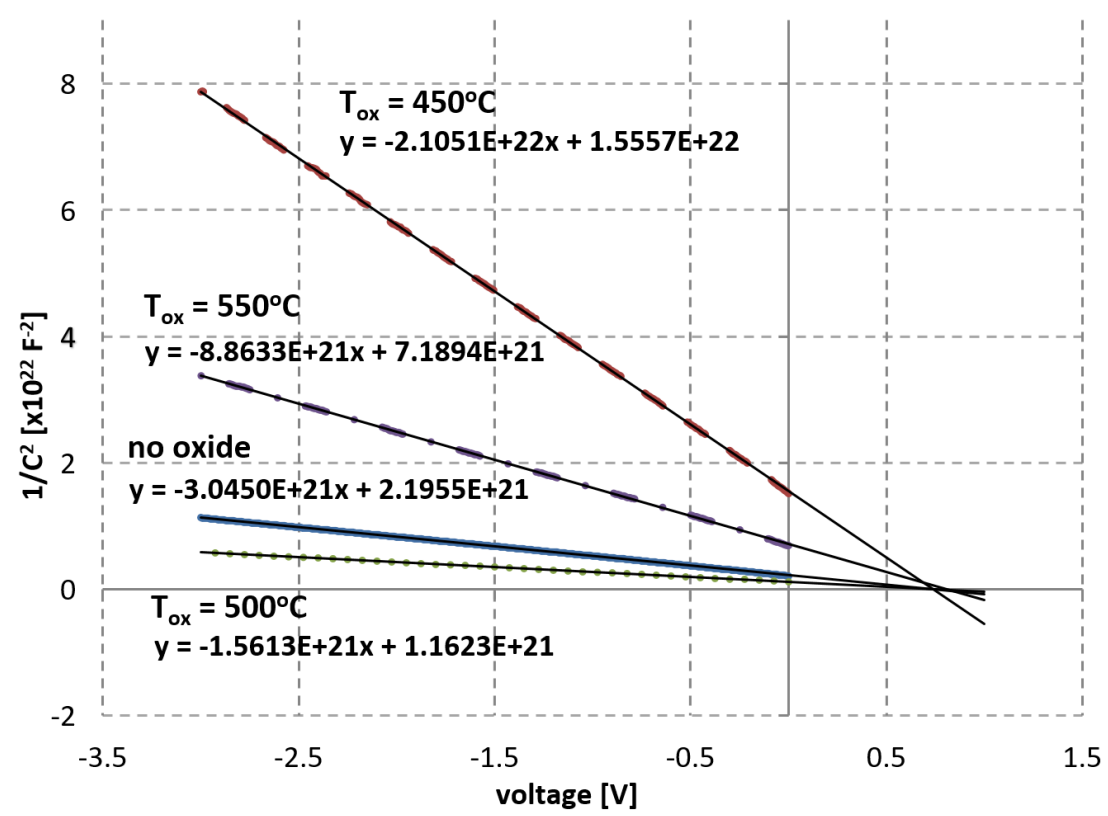

Figure A.1: CV characteristics of several MIS devices with PEDOT:PSS PH750. The device areas vary. The labels indicate the oxidation temperature for interfacial oxide growth and the least squares linear fit to the data.

Table A.1 summarizes the relevant quantities for the extraction of the work function. The work function of PEDOT:PSS PH750, $\phi_{P H 750,0}$ was extracted from the sample oxidized at the highest temperature of $550^{\circ} \mathrm{C}$, since the interface state density is deemed to be lowest in that device as discussed in Chapter 3. The position of the Fermi level below the conduction band, $\phi_{n}$, is calculated from the wafer conductivity, measured with a 4-point probe prior to processing.

\begin{tabular}{c|c|c|c|c|c|}
$\begin{array}{c}T_{o x} \\
{\left[{ }^{\circ} \mathrm{C}\right]}\end{array}$ & $\begin{array}{c}N_{D} \\
{\left[\mathrm{~cm}^{-3}\right]}\end{array}$ & $\begin{array}{c}\phi_{n} \\
{[\mathrm{eV}]}\end{array}$ & $\begin{array}{c}V_{\text {int }} \\
{[\mathrm{V}]}\end{array}$ & $\begin{array}{c}\phi_{b i} \\
{[\mathrm{eV}]}\end{array}$ & $\begin{array}{c}\phi_{m} \\
{[\mathrm{eV}]}\end{array}$ \\
\hline \hline no oxide & $1.73 \mathrm{E}+15$ & 0.2509 & 0.7210 & 0.7469 & 5.05 \\
450 & $1.85 \mathrm{E}+15$ & 0.2492 & 0.7390 & 0.7469 & 5.06 \\
500 & $1.80 \mathrm{E}+15$ & 0.2493 & 0.7444 & 0.7703 & 5.07 \\
550 & $1.85 \mathrm{E}+15$ & 0.2500 & 0.8111 & 0.8370 & 5.14
\end{tabular}

Table A.1: Quantities involved in extracting the conductor work function of MIS diodes from the $\mathrm{CV}$ characteristics. 
The work function of PEDOT:PSS PH750 was calculated as:

$$
\phi_{m}=V_{i n t}+\frac{k_{B} T}{q}+\chi_{S i}+\phi_{n}
$$

\section{A.2 Work function Extraction for Diodes with PE- DOT:PSS/Silica Composite}

The work function is expected to vary between the devices as the composition of the conductor layer varies slightly between devices. The work function values for three of the examined compositions are summarized in Table A.2 and Figure A.2.

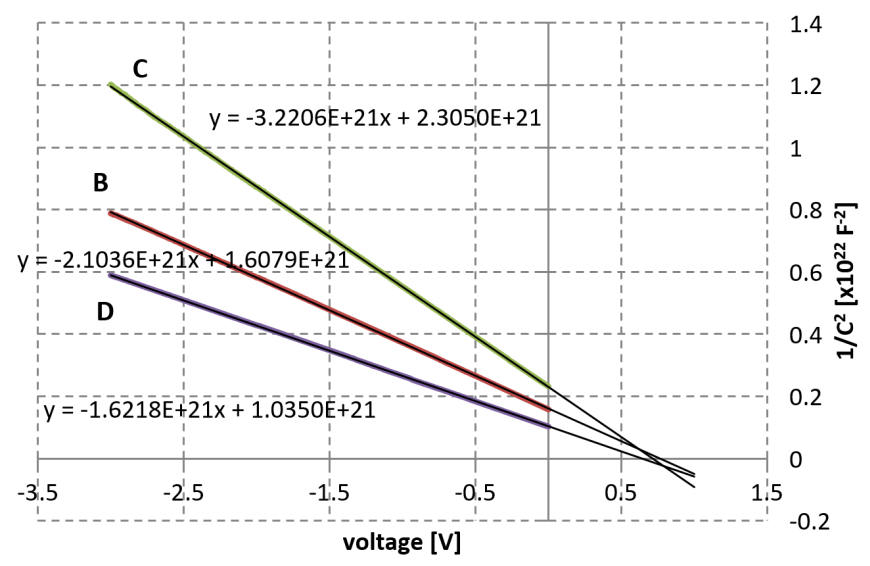

Figure A.2: CV characteristics of Schottky junctions made with $n$-type silicon (1$2 \Omega \mathrm{cm}$ ) and PEDOT/silica composites of slightly different compositions. The areas of the devices are different. 


\begin{tabular}{c|c|c|c} 
Sample & $\begin{array}{c}V_{\text {int }} \\
{[\mathrm{V}]}\end{array}$ & $\begin{array}{c}\phi_{b i} \\
{[\mathrm{eV}]}\end{array}$ & $\begin{array}{c}\phi_{m} \\
{[\mathrm{eV}]}\end{array}$ \\
\hline \hline $\mathrm{B}$ & 0.7644 & 0.7903 & 5.0763 \\
$\mathrm{C}$ & 0.7157 & 0.7416 & 5.0277 \\
$\mathrm{D}$ & 0.6382 & 0.6641 & 4.9501
\end{tabular}

Table A.2: The relevant quantities for extraction the work function of the conductor from the CV characteristics of a Schottky junction. The resistivity of the substrate was taken to be $1.5 \Omega \mathrm{cm}$ for these calculations. This assumption adds an uncertainty of only $0.01 \mathrm{eV}$ to the extracted work function value. 


\section{Appendix B}

\section{Computation of Reflectivity for} Nanostructured Silicon Substrates

To calculate the reflectivity of a structured substrate according to the effective medium theory as described in Chapter 2, it is necessary to know the permittivity of the substrate material and the geometry of the nanostructure. The geometry was assumed to be ideal, i.e. a forest of cones with the volume fraction of silicon varying smoothly from $100 \%$ at the substrate interface to $0 \%$ at the ambient interface.

The dispersive nature of $\mathrm{Si}$ was modelled by a complex permittivity expressed as a sum of two harmonic oscillators as expressed by equation B.1 [149]. The harmonic oscillator parameters were extracted from a numerical fit performed in MATLAB to data acquired from PVCDROM [7]. The fit is shown in Figure B.1. The parameters extracted from the fit (listed in Table B.1) were used in the numerical evaluation of reflectance of nanostructured surfaced.

$$
\epsilon=\epsilon_{\infty}+\sum_{n=1}^{2} \frac{\sigma_{n} f_{n}^{2}}{f_{n}^{2}-f^{2}-i f \gamma_{n}}
$$




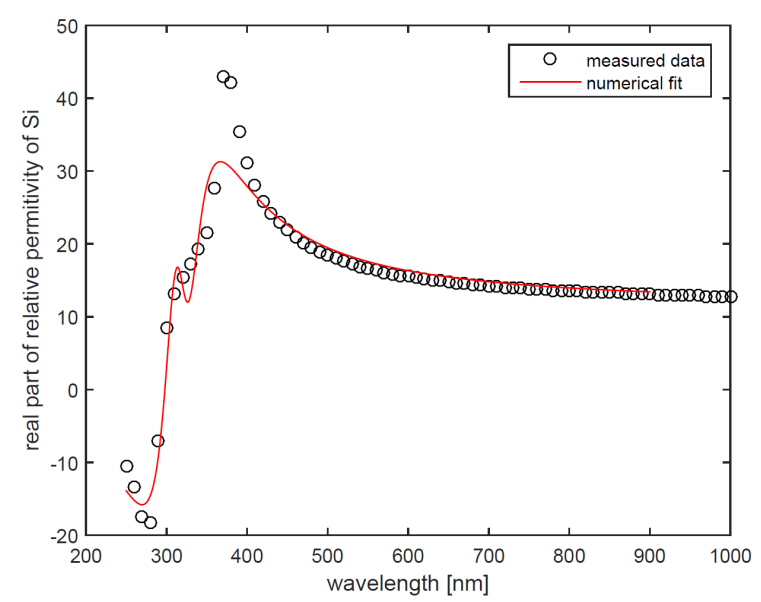

(a)

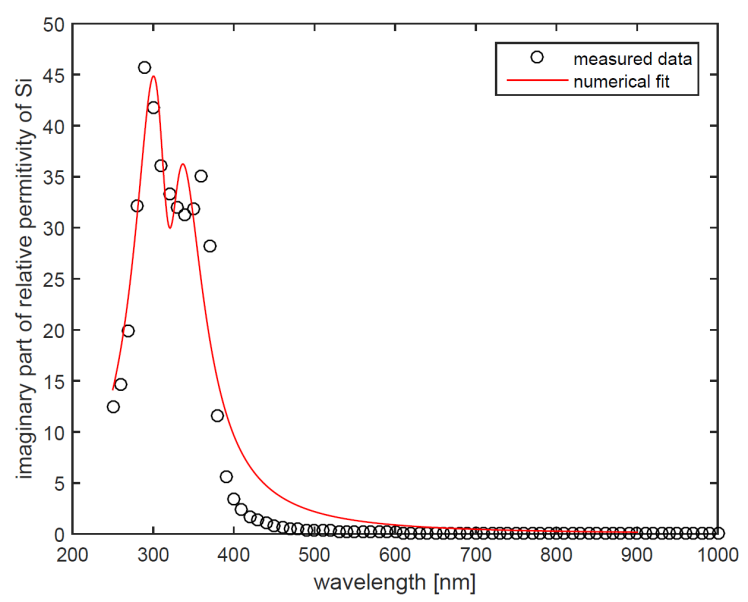

(b)

Figure B.1: Numerical fit for the complex permittivity of silicon modelled as a sum of two harmonic oscillators (a) real part of the relative permittivity (b) imaginary part of the relative permittivity.

\begin{tabular}{c|c}
\hline$\epsilon_{\infty}=11.6833$ & \\
$\sigma_{n_{1}}=-189.5980 \mathrm{~nm}^{-2}$ & $\sigma_{n_{2}}=177.3352 \mathrm{~nm}^{-2}$ \\
$\mathrm{f}_{n_{1}}=319.0016 \mathrm{~nm}^{2}$ & $\mathrm{f}_{n_{2}}=319.0735 \mathrm{~nm}^{2}$ \\
$\gamma_{n_{1}}=-52.5811 \mathrm{~nm}$ & $\gamma_{n_{2}}=-50.5326 \mathrm{~nm}$ \\
\hline
\end{tabular}

Table B.1: Parameters of the harmonic oscillator model for the permittivity of silicon when the wavelength is expressed in $\mathrm{nm}$. 
The following is the recursive code used for evaluating reflectivity of the nanostructured silicon surface.

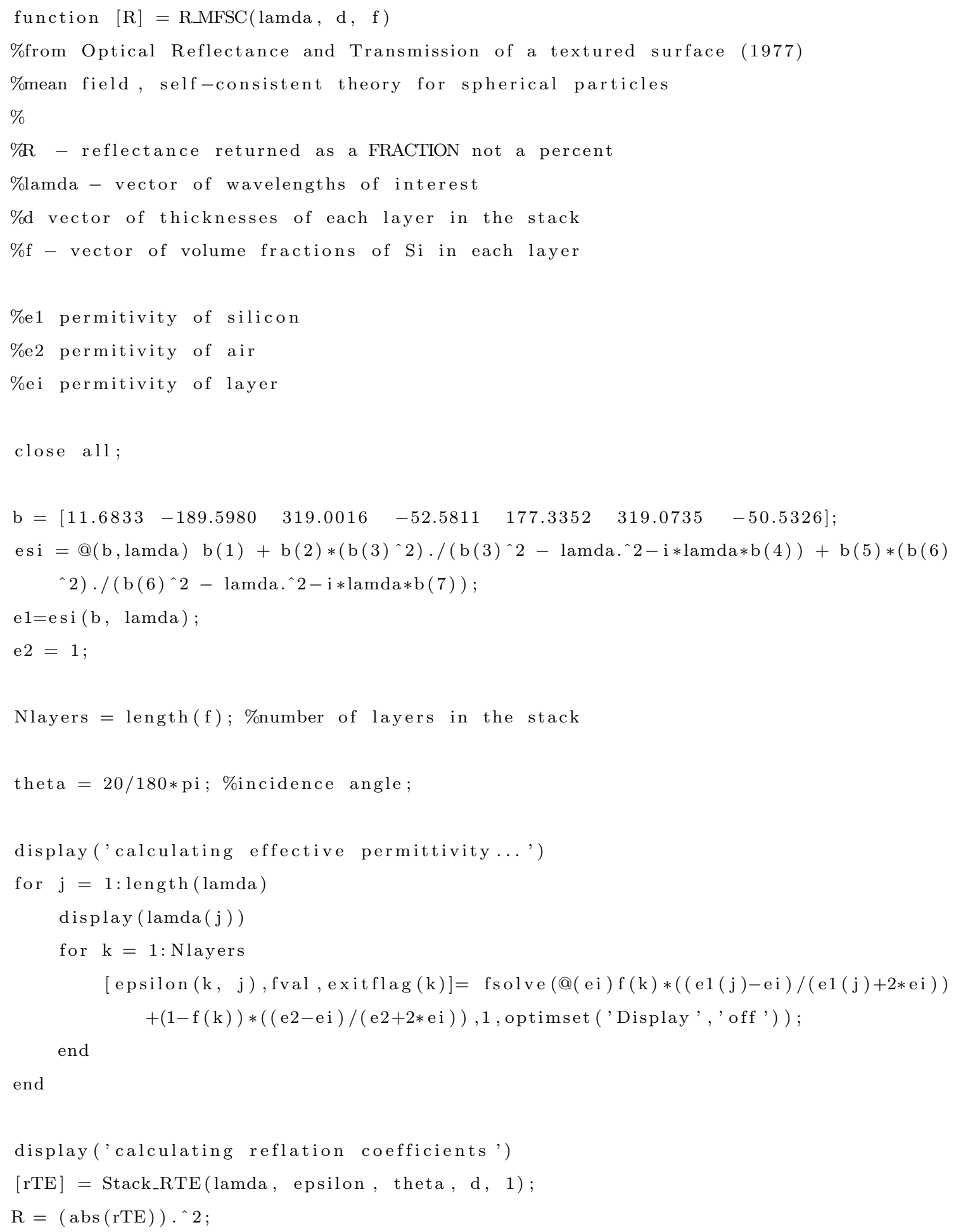




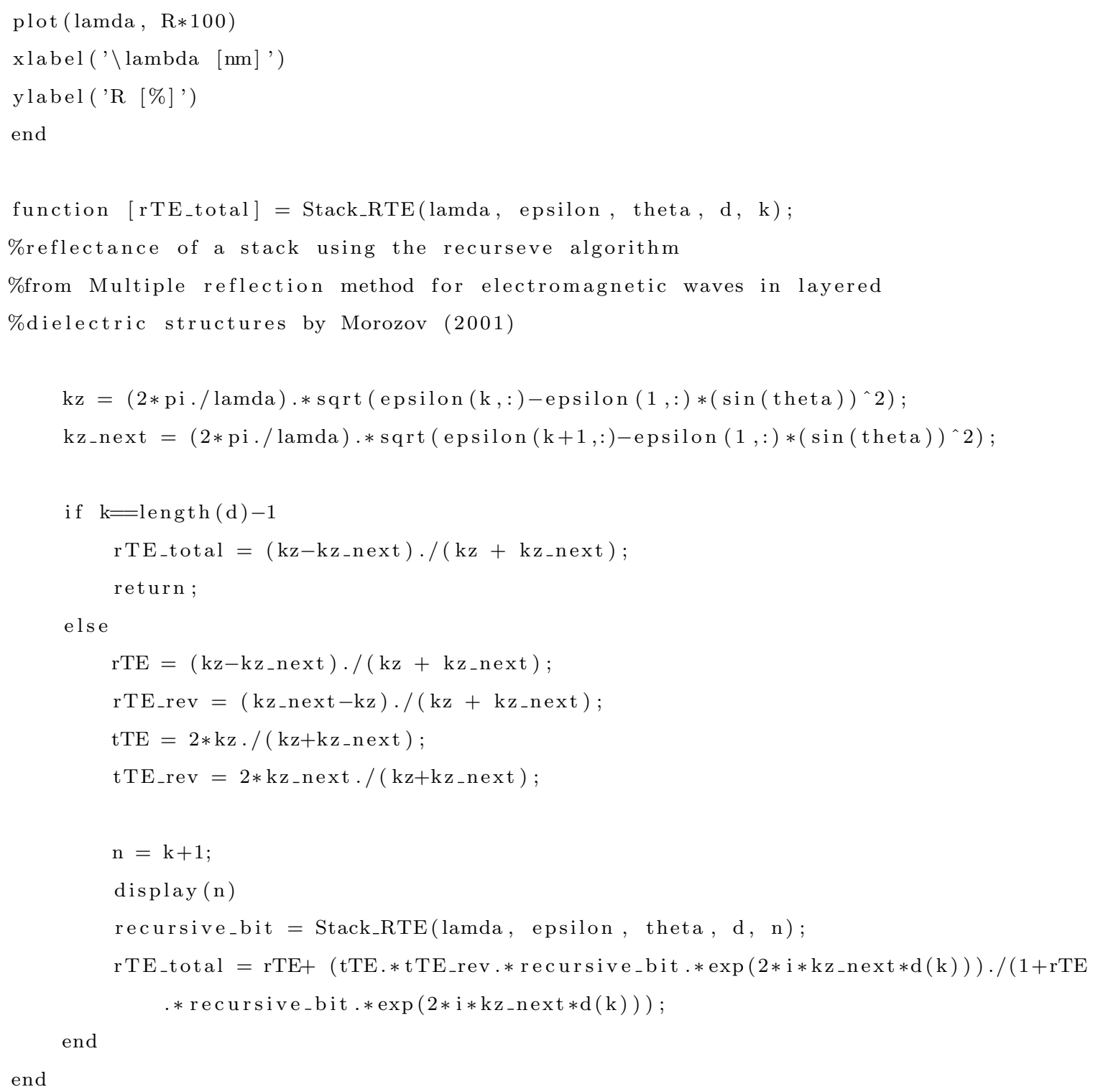




\section{Appendix $\mathrm{C}$ \\ PlasmaTherm SLR-772 ECR Etcher Recipe for Black Silicon.}




\begin{tabular}{l|c|l} 
Parameter & Value & Comment \\
\hline \hline Etch time & $20-30 \mathrm{~min}$ & \\
\hline Microwave forward power & Approx. 260 W & $\begin{array}{l}\text { Set maximum microwave } \\
\text { forward power to } 350 \mathrm{~W} \text { and } \\
\text { adjust it during etch to get } \\
1-2 \mathrm{~W} \text { of reflected power }\end{array}$ \\
\hline Microwave reflected power & $1-2 \mathrm{~W}$ & \\
\hline $\mathrm{RF}$ power & $10 \mathrm{~W}$ & \\
\hline $\mathrm{RF}$ reflected & $0 \mathrm{~W}$ & \\
\hline Microwave tuning stubs & Stub $3-6.27$ & \\
& Stub $2-8.77$ & \\
\hline SF $F_{6}$ flow rate & Vertical -80 & \\
\hline $\mathrm{O}_{2}$ flow rate & 3.65 sccm & \\
\hline He set point & $6.78 \mathrm{sccm}$ & \\
\hline Gas pressure & 4.5 & Setting for chuck cooling \\
\hline He leakage (on) & $6 \mathrm{mTorr}$ & \\
\hline Chuck temperature & Approx. $1-5 \mathrm{mTorr}$ & Observed leakage \\
\hline
\end{tabular}

Table C.1: PlasmaTherm SLR-772 ECR etcher recipe for black silicon. 


\section{Appendix D}

\section{Increase in Recombination Current due to Surface Nanostructuring}

The recombination current, $J_{r}$, in the diode is proportional to the interface state density, $D_{i t}$, through a quantity known as the "surface recombination velocity", $S$, as described by equations 2.12 and 2.13 in Chapter 2. These equations are reproduced below for convenience. Here $v_{t h}$ is the thermal velocity, $\sigma$ is the capture cross section area, and $\Delta p$ is the excess minority hole carrier density at the interface for an $n$-type substrate. The lowest interface state density found in literature was for a thermally grown oxide at a value of $N_{s}=1 \times 10^{9} \mathrm{~cm}^{-3}$, resulting in recombination velocity of $S=11.45 \mathrm{~cm} / \mathrm{s}$. Using simulation parameters that best fit the behaviour of the tested inorganic diode with gold (see Chapter 6) the hole concentration at the interface under one sun illumination was simulated to be approximately $2 \times 10^{15} \mathrm{~cm}^{-3}$. This gives an expected recombination current for a flat diode of $J_{r}=1.8 \mathrm{~mA} / \mathrm{cm}^{2}$.

$$
\begin{gathered}
J_{r}=q U_{s} \\
U_{s}=\frac{S p_{g}}{2}=\frac{\sigma v_{t h} \overline{D_{i t}} E_{G} p_{g}}{2}
\end{gathered}
$$

In a device with a nanostructured surface the effective surface state density would 


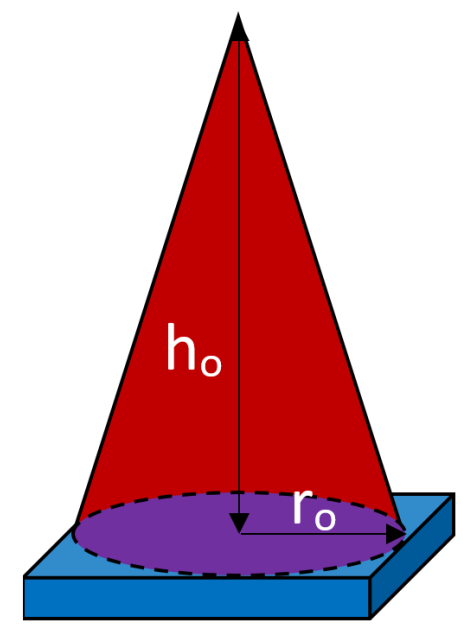

Figure D.1: Effective surface area increase due to nanostructuring for reflection minimization.

increase proportionally with the effective area of the junction. This area increase is estimated based on the ideal geometry for minimizing surface reflections, as this is the purpose of the nanostructure. Figure D.1 defines a unit cell of the structured surface. According to this geometry, the flat and structured areas are given by equations D.3 and D.4 respectively. The effective area increase is the ratio between the structured and the flat areas and can be expressed in terms of the given geometry as shown in equation D.5. Assuming optically favourable and practically achievable values for the structure dimensions of $h_{o}=1000 \mathrm{~nm}$ and $r_{o}=150 \mathrm{~nm}$, the area can be estimated to increase by a factor of 5.5. This five-fold increase in area will proportionally increase the recombination current to $J_{r}=10 \mathrm{~mA} / \mathrm{cm}^{2}$.

$$
\begin{gathered}
A_{\text {flat }}=4 r_{o}^{2} \\
A_{\text {structured }}=(4-\pi) r_{o}^{2}+\pi r_{o} \sqrt{h_{o}^{2}+r_{o}^{2}} \\
\text { ratio }=1-\frac{\pi}{4}\left(1-\sqrt{\left(\frac{h_{o}}{r_{o}}\right)^{2}+1}\right.
\end{gathered}
$$




\section{Appendix E}

\section{Optimal Resistivities/Transparencies for two Phase filling of Nanostructured Substrates with PEDOT/Silica}

As all the formulations of PEDOT have a finite transparency, the filling of a large aspect ratio nanostructured surface can effectively block most of the light from reaching the substrate. This effect will be called "PEDOT shading". It can drastically reduce cell efficiency. To minimize the effect of this, a nanostructured substrate needs to receive two coatings of different PEDOT formulations as shown in Figure E.1. The top PEDOT layer can be thin so shading would not be a significant issue. It should be optimized for best conductivity for effective charge transport to the finger grid for collection. The PEDOT penetrating the nanostructure should be optimized such that the power losses from PEDOT shading and resistivity of PEDOT are balanced, because there is always a trade-off between transparency and conductivity.

As proposed in Chapter 3, the best material to use for filling a nanostructured surface is a PEDOT/silica composite. Not only does it show excellent adhesion to a silicon surface, but it also demonstrates the ability to effectively and completely fill-in the nanostructure, as opposed to some other formulations. Additionally, the relative 


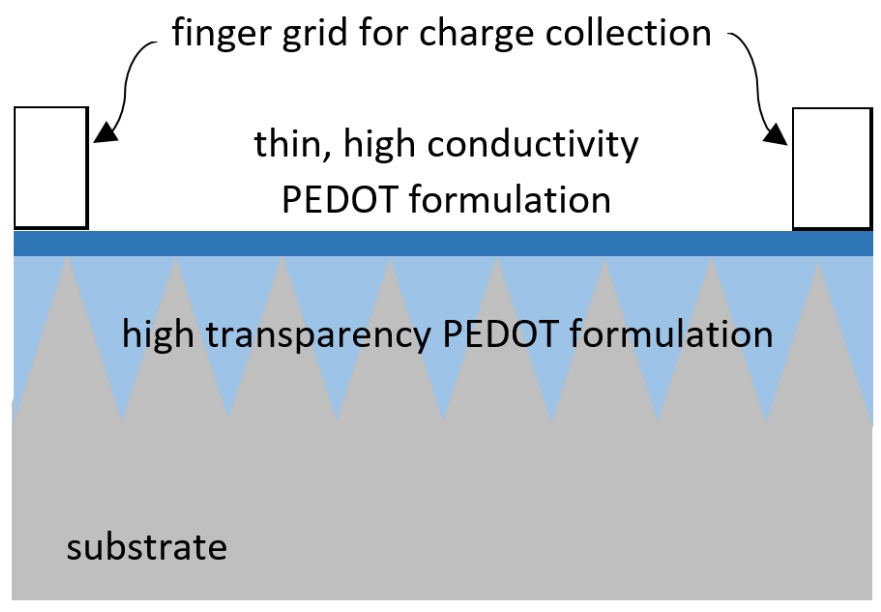

Figure E.1: Sketch of a nanostructured solar cell that uses two types of PEDOT: one for best optical transparency without compromising the transition of charge up to the top of the nanostructure and another, more conductive, thin layer for horizontal transmission of charge to the finger grid electrodes for charge collection.

content of silica in the mixture can be used to tune the transparency/conductivity balance in relatively thick films. This appendix details a procedure for estimating the desired conductivity of PEDOT/silica composite for filling a nanostructured surface given the ideal geometry for minimizing surface reflection, i.e. a forest of cones. Figure E.2 shows a unit cell of this structure. As discussed in Chapter 4, a height of $h_{o}=1000 \mathrm{~nm}$ and feature size of $2 r_{o}=300 \mathrm{~nm}$ are reasonable to use for these estimations.

Assuming that most of the photocurrent is generated in the bulk of the substrate, the current path will be upwards from the substrate through the PEDOT. Any current contributions generated or transported through the cone are ignored. In this case the PEDOT resistance can be expressed as shown in equation E.1

$$
R_{P E D O T}=\int_{0}^{h_{o}} \frac{\rho}{A} d h=\rho \int_{0}^{h_{o}} \frac{1}{4 \rho_{o}^{2}-\pi\left(\frac{\rho_{o}\left(h_{o}-h\right)}{h_{o}}\right)^{2}} d h
$$

Using the identity $\sin ^{2} x+\cos ^{2} x=1$, this expression can be evaluated as shown 


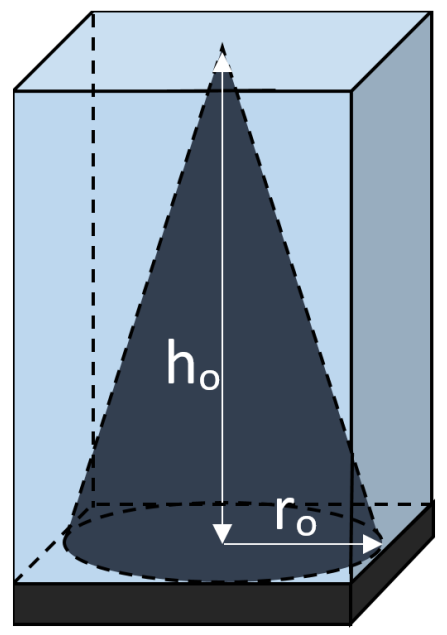

Figure E.2: Geometry for estimating PEDOT resistivity.

in equation E.2.

$$
R_{P E D O T}=\frac{\rho 2 h_{o}}{4 \rho_{o}^{2} \sqrt{\pi}}\left[-\left.\ln |\csc x+\cot x|\right|_{\arccos \frac{\sqrt{\pi}}{2}} ^{\arccos 0}\right]
$$

The power losses to balance for optimum solar cell performance are electrical loss due to the resistance of the PEDOT composite (equation E.3) and optical loss due to PEDOT shading (equation E.4) These two quantities are related through the interdependence of resistivity and transparency of PEDOT/silica composite. Both can be expressed as a function of the ratio between the PEDOT and sol-gel silica by weight, $\xi$ (equations E.5, E.6).

$$
\begin{gathered}
P_{\text {loss }, \text { elec }}=I_{L}^{2} R=\left(A J_{\text {Lmax }} T\right)^{2} R_{\text {PEDOT }} \\
P_{\text {loss }, \text { opt }}=A J_{\text {Lmax }}(1-T) V_{o c} F F \\
T_{o p t}=e^{-\alpha t_{P E D O T}} ; \alpha \propto \xi \\
\sigma \propto \xi
\end{gathered}
$$

The dependence of the attenuation coefficient, $\alpha$, and conductivity, $\sigma$, on the ratio 
between the PEDOT and sol-gel silica by weight, $\xi$, is derived from data published by Lee, Lim and Son [124] using percolation theory. For a large matrix of randomly interspersed conductive and isolating domains, conductivity is proportional to a quantity termed "connectivity length", $l_{c}$, as defined below:

$$
\sigma \propto l_{c}^{-\mu}=\left(-\frac{1}{\ln (p)}\right)^{-\mu}
$$

where $p$ is the probability of a domain being conductive in the percolation matrix. The ratio of PEDOT to silica is a good estimate of this probability. The conductivity exponent, $\mu$, is reported to be near 1 for 2-dimensional systems, and 2.3 for 3-dimensional systems. For systems close to the percolation threshold, $p_{c}$, the connectivity length can be simplified to [150]:

$$
l_{c}=-\frac{1}{\left(p-p_{c}\right)}
$$

The conductivity and transmission data published by Lee are reproduced in Figure E.3. The attenuation coefficient was not reported by Lee explicitly, but was estimated from his available data ${ }^{1}$. The data measured for our composite films are also included in the figures but were not included in estimating the lines of best fit. Some variation in both transparency and conductivity is expected as the oxidation environment is not likely to be the same, because the ambient conditions for Lee's oxidation process were not reported. It is known in the literature that the ratio of the EDOT monomer to the dopant, ambient temperatures, as well as the humidity affect the doping efficiency of the resultant PEDOT film, which, in turn, determines conductivity and transparency

\footnotetext{
${ }^{1}$ The attenuation coefficient for the composite with the PEDOT to silica ratio of $\xi=0.8$ was extracted from the transmission vs composite thickness data (Figure E.3(c)), and is $\alpha=2.7861 \times 10^{-3} / \mathrm{nm}$. Based on the data from Figure E.3(b) this corresponds to a transmission of $\mathrm{T}=62.1 \%$, giving a film thickness of approximately $171 \mathrm{~nm}$. Assuming the same thickness for all the films with various PEDOT to silica ratios, the attenuation coefficient as a function of the ratio was calculated from Lee's transmission vs ratio data (Figure E.3(b)).
} 
[62]. The best fit to the conductivity data is consistent with percolation theory for a three-dimensional matrix near percolation threshold (Figure E.3(a)) The conductivity appears to saturate near $\xi=1$. This observation is consistent with finite conductivity of in situ PEDOT. The best fit to the attenuation coefficient data is consistent with percolation theory for a two-dimensional matrix below percolation threshold (Figure E.3(d)).

This information allows the computation of the expected electrical and optical losses as a function of the PEDOT to silica ratio according to equations E.3 and E.4. The power loss is calculated as a fraction of maximum possible generated power in a solar cell with moderate open circuit voltage and fill factor of $0.536 \mathrm{~V}$ and 0.66 [71]. The minimum loss of $0.0005 \%$ is achieved for a $1 \mu \mathrm{m}$ deep nanostructure with a PEDOT to silica ratio of 0.14 . 


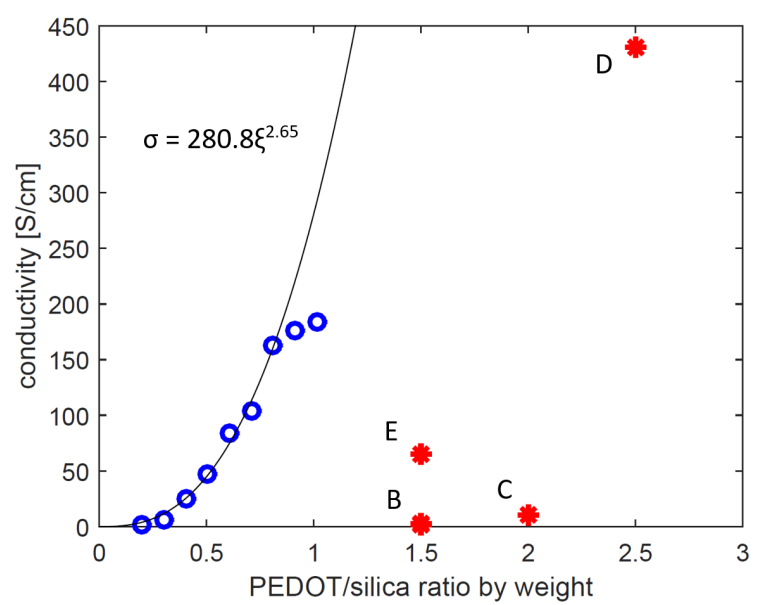

(a)

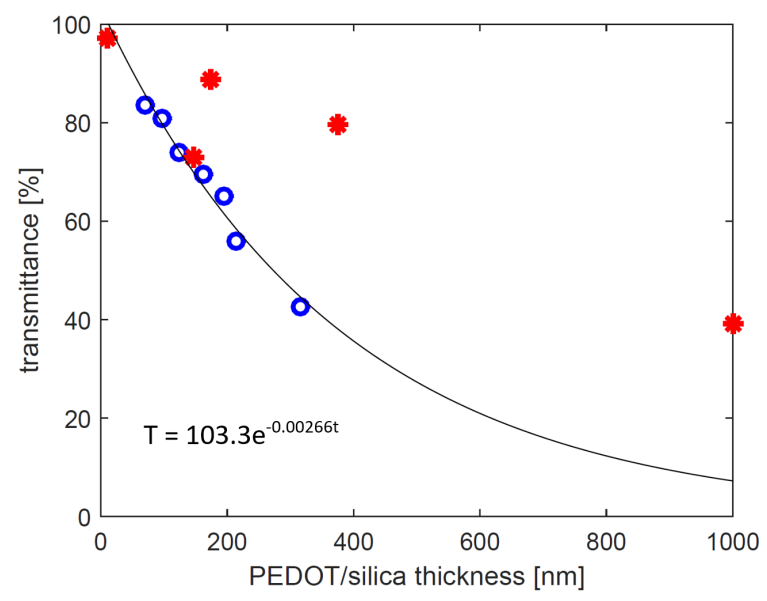

(c)

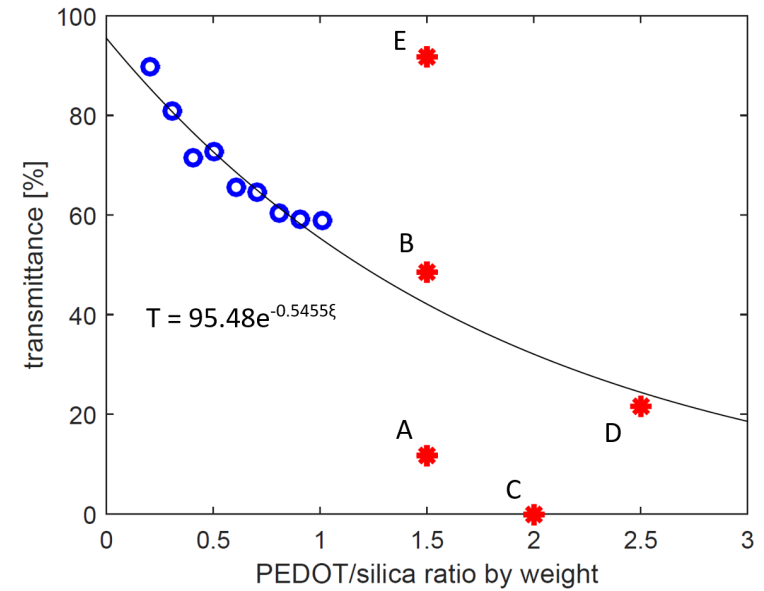

(b)

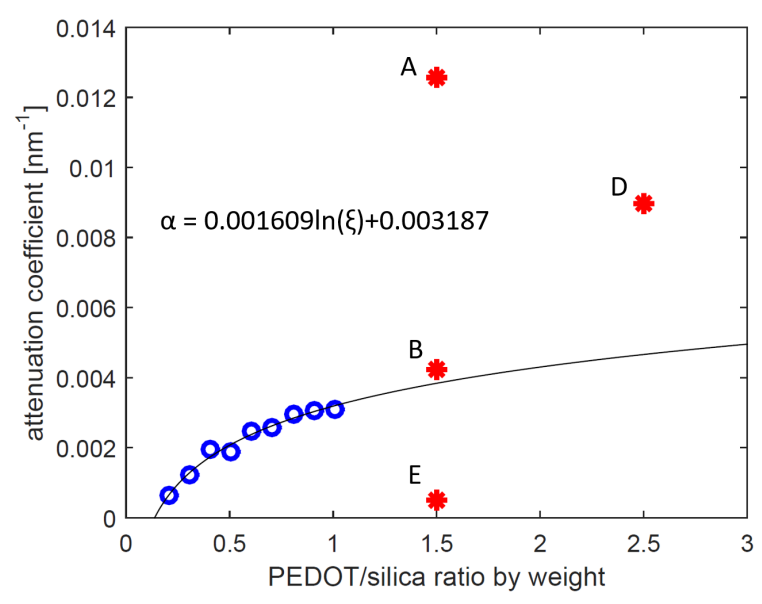

(d)

Figure E.3: Properties of PEDOT/silica composites as reported by Lee (blue); results obtained in this work (sample IDs are defined in Chapter 3) are included for reference but were not used to calculate the best fits (red) (a) conductivity vs PEDOT content for composites with EDOT/FTS $=1 / 2.25$ mol ratio (b) transmittance vs PEDOT content for composited with EDOT/FTS $=1 / 2.25 \mathrm{~mol}$ ratio (c) transmittance vs $\mathrm{PEDOT}$ thickness for composites with PEDOT ratio of 0.8 . 


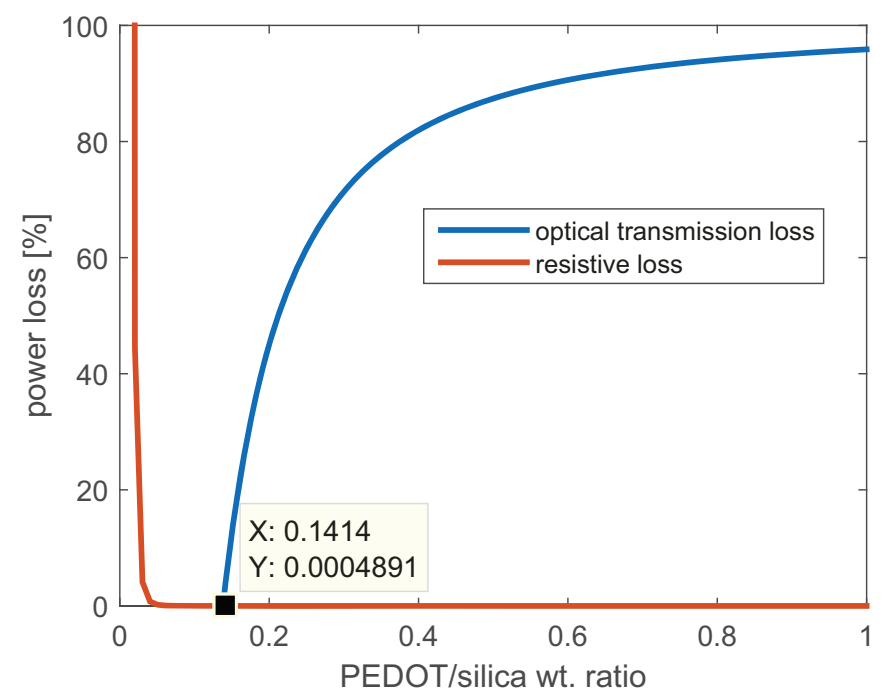

Figure E.4: Power loss optimization for PEDOT/silica filled nanostructure $1000 \mathrm{~nm}$ high based on the composite parameters of Lee et al. [124]. 


\section{Appendix F}

\section{A Comparative Study of Minimum Power Loss for the Planarized and Structured Devices}

The merits of planarizing a nanostructure with high transparency PEDOT before applying a high conductivity current collection layer vs conformally coating the nanostructure are assessed here. The illustrations of the two proposed schemes are reproduced in Figure F.1 for reference. Since the power loss due to the structure filling (Figure F.1(a)) can be minimized to $0.0005 \%$, only the thin, high-conductivity PEDOT layers will be considered here.

The competing losses to be minimized are the optical losses due to the finger grid shadowing and the transparancy of the thin PEDOT layer. The finger grid shadowing is a function of finger spacing, $s_{f}$, and finger width, $w_{f}$. The finger width will be held constant in this analysis at a reasonable value of $100 \mu \mathrm{m}$ [9]. The finger spacing will be varied. The further the current-collecting fingers are apart, the smaller this loss. However, large finger spacing increases the resistive loss. The largest PEDOT conductivity found reported to date is $1485 \mathrm{~S} / \mathrm{cm}$ for vapour phase polymerized (VPP) material [125]. As such, this value will be used in calculations. 


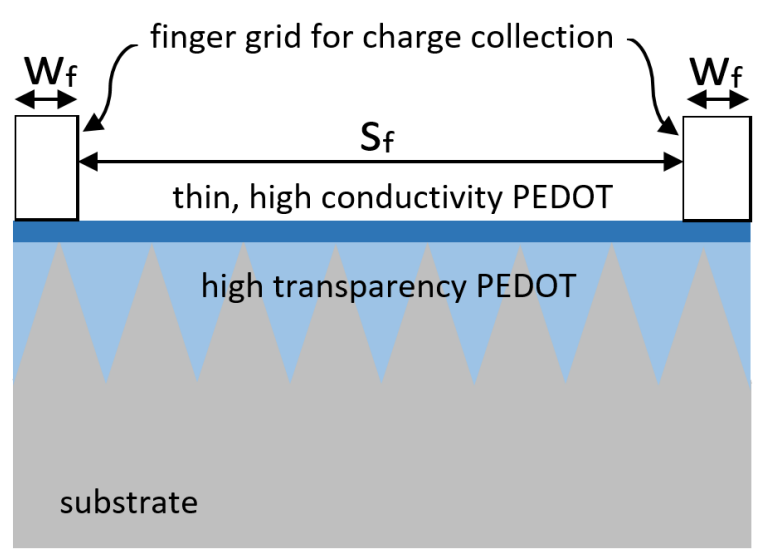

(a)

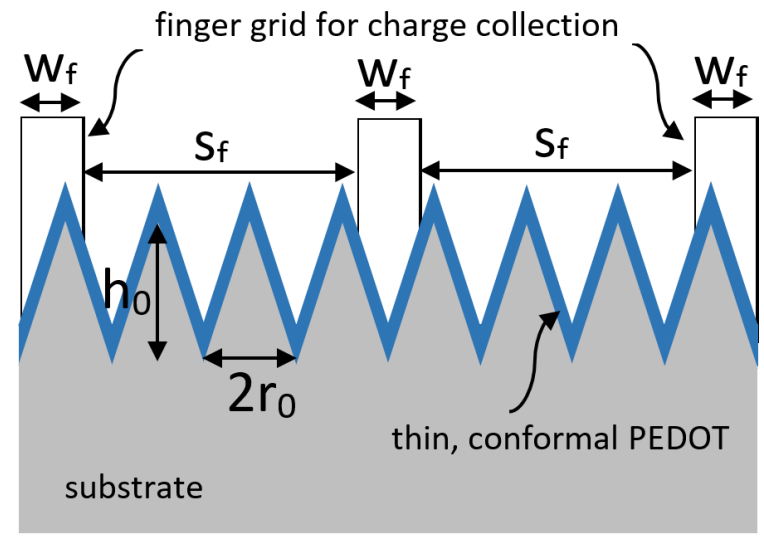

(b)

Figure F.1: PEDOT deposition schemes for solving the PEDOT shading problem (a) two types of PEDOT: one for best optical transparency without compromising the transition of charge up to the top of the nanostructure and another, more conductive, thin layer for horizontal transmission of charge to the finger grid electrodes for collection. (b) thin highly conductive conformal layer of PEDOT; the spacing of the finger grid is limited by the conductivity of PEDOT.

VPP PEDOT is essentially in situ PEDOT. The light attenuation will be generously underestimated by using the smallest attenuation coefficient reported by H.C. Starck of $\alpha=0.001741 \mathrm{~nm}^{-1}$ (at $\left.\lambda=350 \mathrm{~nm}\right)$.

The optical loss consists of light blocked by the finger grid and the attenuation due to PEDOT where there is no grid.

$$
P_{\text {loss }, o p t}=\frac{w_{f}}{w_{f}+s_{f}}+\frac{s_{f}}{w_{f}+s_{f}}\left(1-T_{o p t}\right)
$$

$T_{\text {opt }}$ is the optical transmission defined as:

$$
T_{\text {opt }}=e^{-\alpha t_{P E D O T}}
$$

The electrical loss is due to PEDOT resistivity normalized by maximum possible generated power. However, the current path length is much larger in the case of a structured surface: 


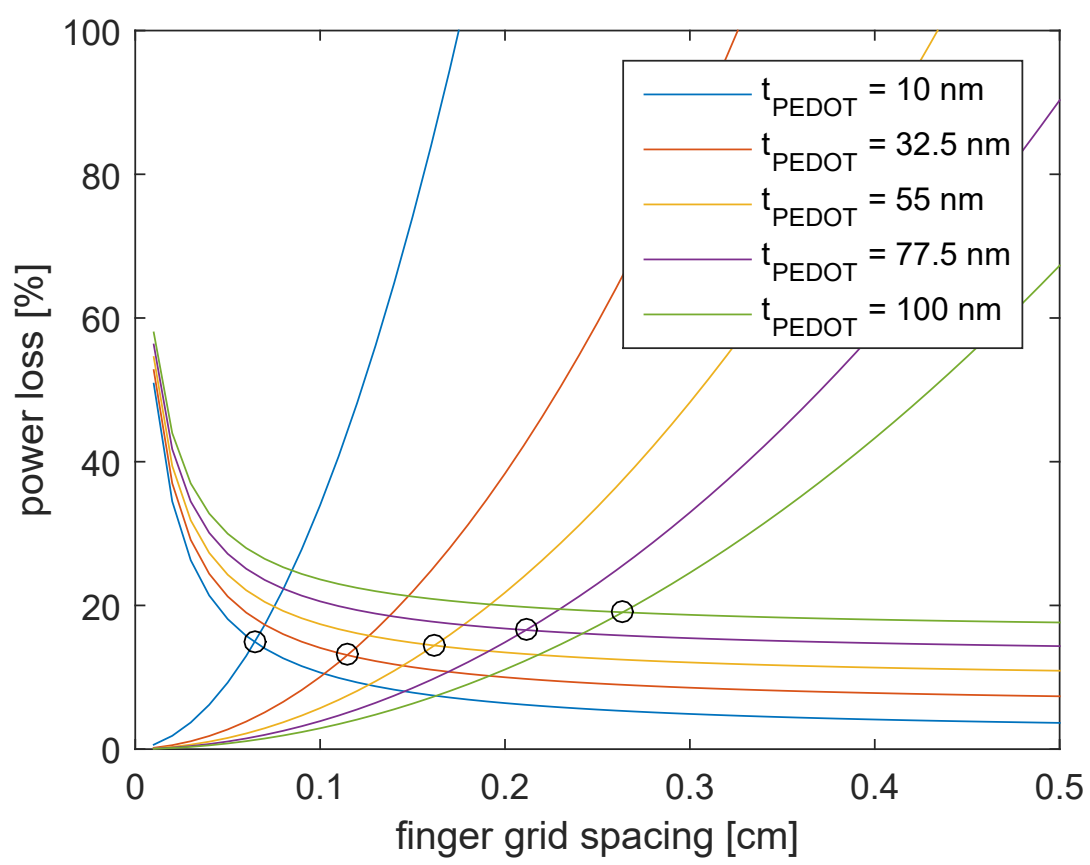

Figure F.2: Series of optical and resistive loss curves as function of finger grid spacing for various PEDOT thicknesses. The minimum power loss for each PEDOT thickness is indicated with an "o".

$$
\begin{gathered}
P_{\text {loss }, \text { elec }, \text { flat }}=\frac{\left(s_{f}+w_{f}\right) J_{\max } \rho_{P E D O T}}{V_{o c} F F t_{P E D O T}} \frac{s_{f}}{2} \\
P_{\text {loss }, \text { elec }, \text { struct }}=\frac{\left(s_{f}+w_{f}\right) J_{\max } \rho_{P E D O T}}{V_{o c} F F t_{P E D O T}} \frac{s_{f}}{2} \frac{\sqrt{r_{o}^{2}+h_{o}^{2}}}{r_{o}}
\end{gathered}
$$

For every PEDOT thickness, there exists a finger grid spacing that minimizes power loss. These were found for a range of VPP PEDOT thicknesses. Figure F.2 illustrates this loss minimization procedure for a planarised device.

The results of the minimization are shown in Figure F.3. The planarized geometry outperforms the conformal coating on the structured surface with a minimum power loss of approximately $13 \%$ with $26 \mathrm{~nm}$ thick PEDOT and $0.1 \mathrm{~cm}$ finger grid spacing as compared to a minimum loss of $20 \%$ (with PEDOT thickness of $41 \mathrm{~nm}$ and grid 


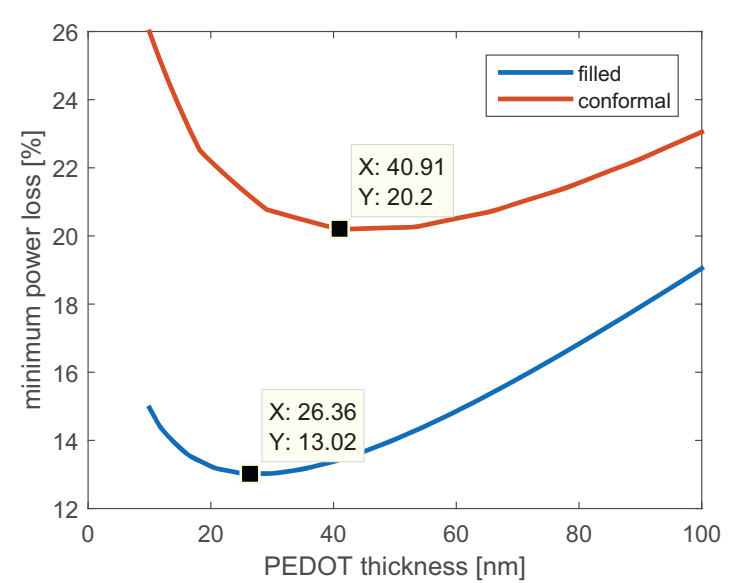

(a)

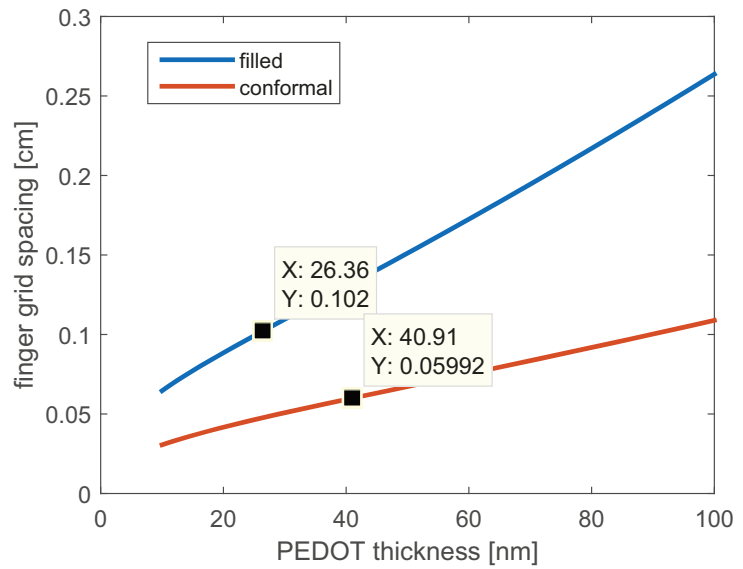

(b)

Figure F.3: Surface structure parameters for minimizing power loss with $100 \mu \mathrm{m}$ wide current collection fingers and $1485 \mathrm{~S} / \mathrm{cm}$ PEDOT. (a) minimum power loss achieved with optimal finger spacing (b) optimal finger spacing for minimizing power loss.

spacing of $0.06 \mathrm{~cm}) .{ }^{1}$ However, reflective losses should be taken into account. The simulation results for the reflectivity of the filled and unfilled $1 \mu \mathrm{m}$-deep structure are shown in Figure F.4. Optical properties reported by H.C. Stark for PH500 were used to calculate its permittivity used in these simulations [151]. The reflectivity of the empty structure is expected to be below $0.5 \%$, whereas filling the structure with PEDOT raises the reflectivity to about 3\%. Even with this additional power loss, the planarized geometry can be expected to outperform the conformal coating based on the analysis presented here.

\footnotetext{
${ }^{1}$ Lower losses may be achievable with thinner fingers, but the tendency of the flat structure to perform better would be unchanged.
} 


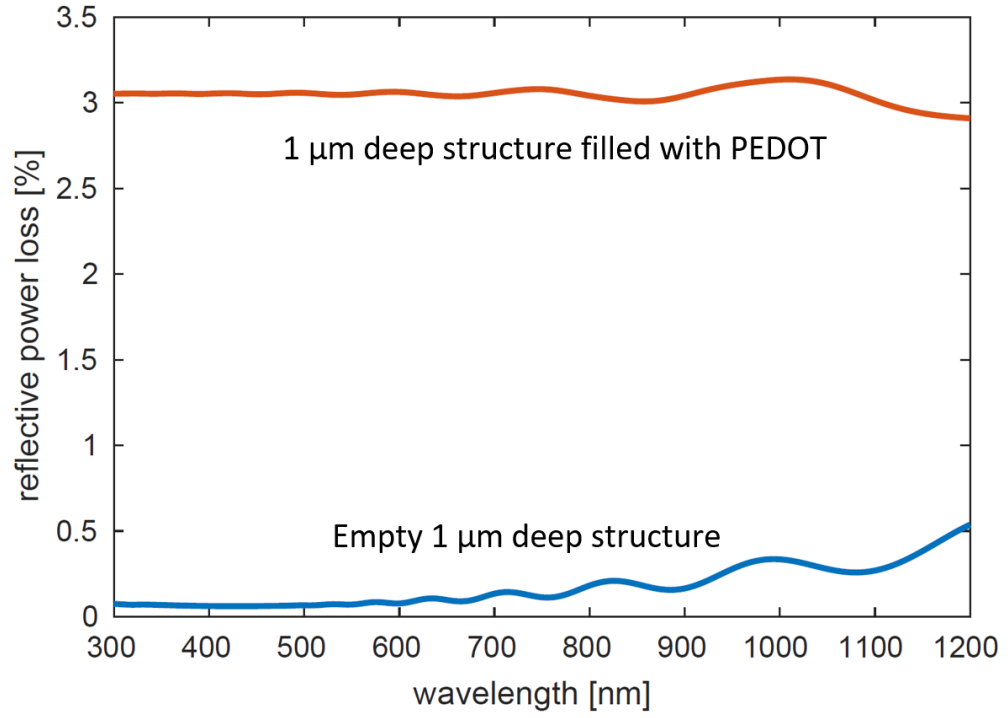

Figure F.4: Simulated reflectivities of a triangular silicon structures $1 \mu \mathrm{m}$ deep. One is empty and the other is filled with PEDOT. 


\section{Appendix G}

\section{MATLAB Code for the MIS Semiconductor Solver}

This code uses one external function for determining an intercept between two curves, defined by vectors of $\mathrm{x}$ and $\mathrm{y}$ co-ordinates. This function was written by Douglas M. Schwarz and is available through Matlab Central under the name "intersections.m" [152].

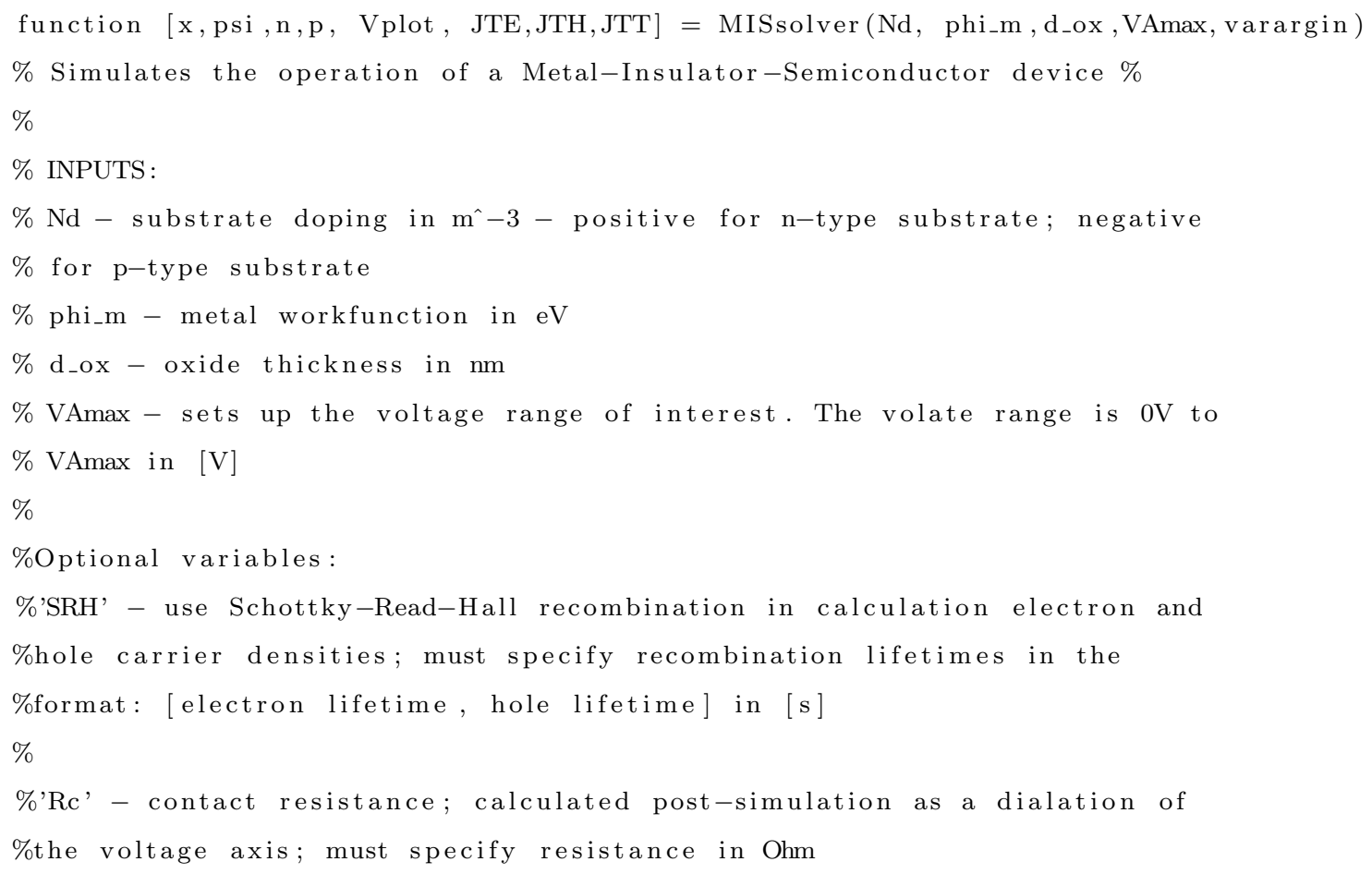




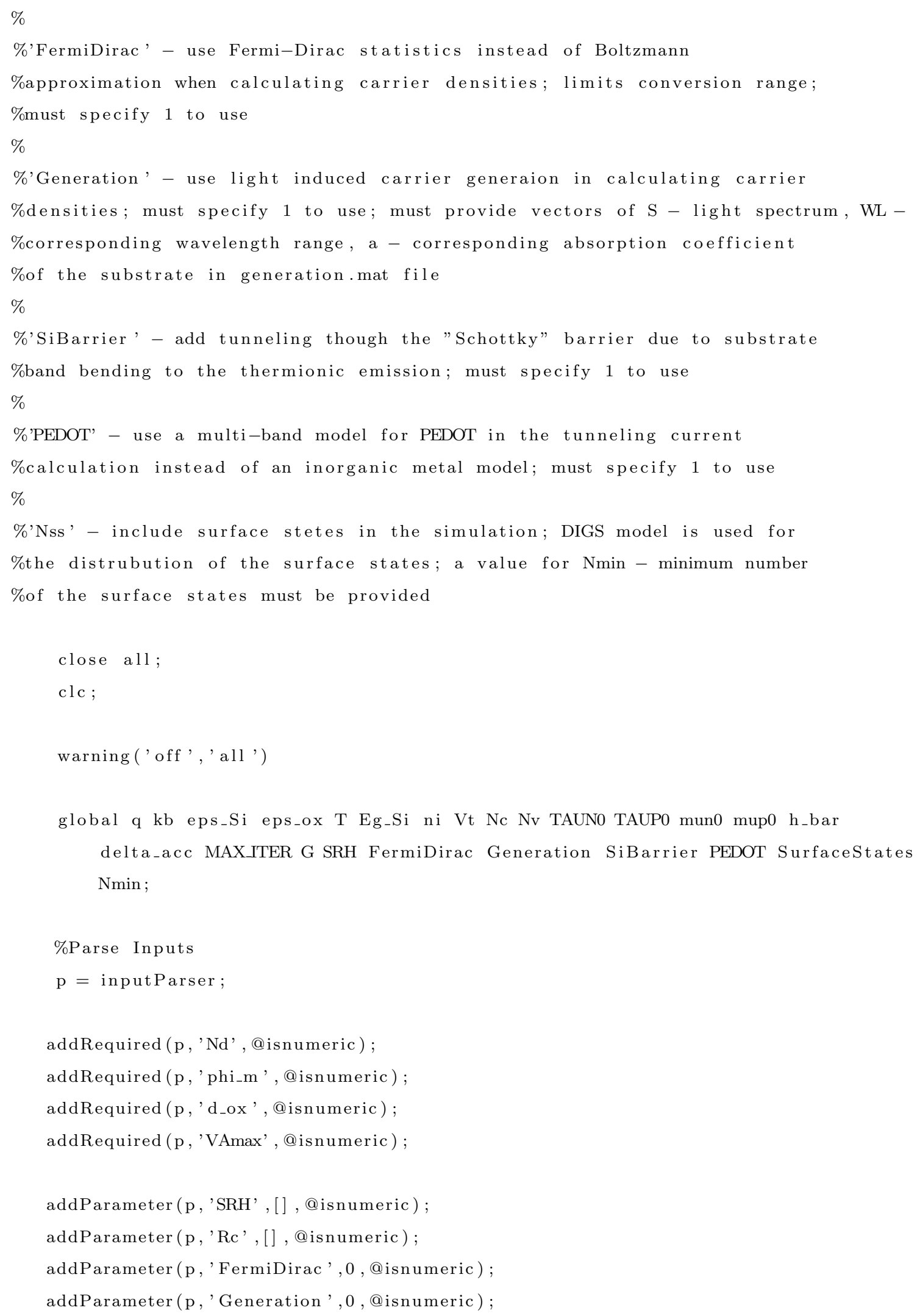




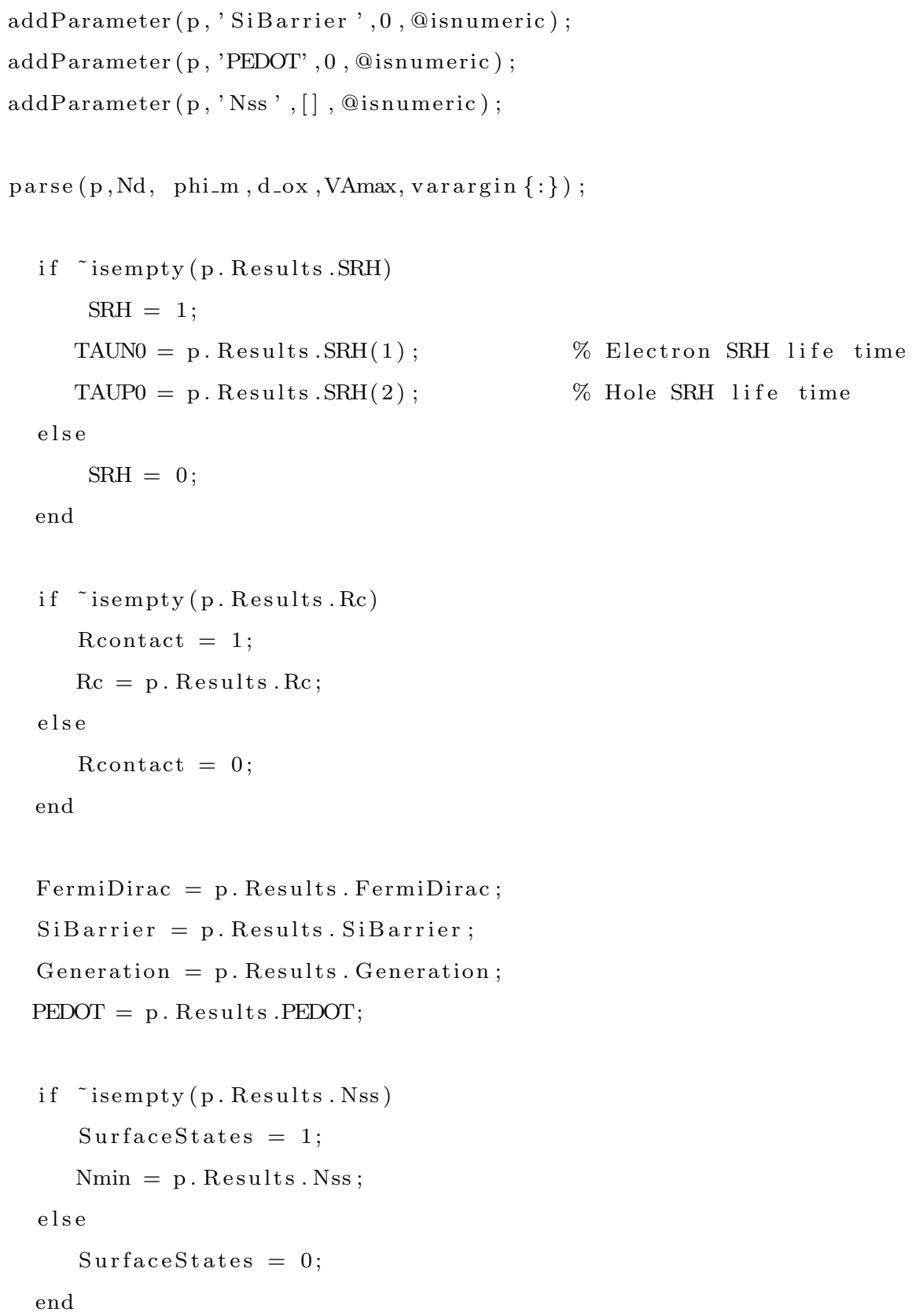
$\mathrm{T}=300 ;$
$\%[\mathrm{~K}]$

DefineGlobal ();

\%DEFINE SIMULATION PARAMETERS

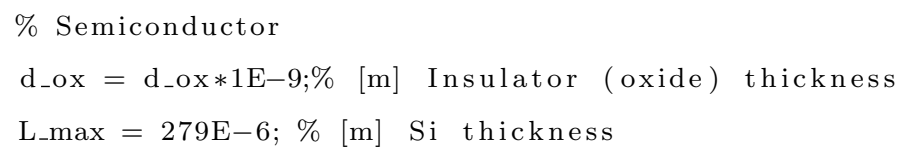


chi_s $=4.05 ; \quad \%[\mathrm{eV}]$ electron affinity of $\mathrm{Si}$

if $\mathrm{Nd}>0$

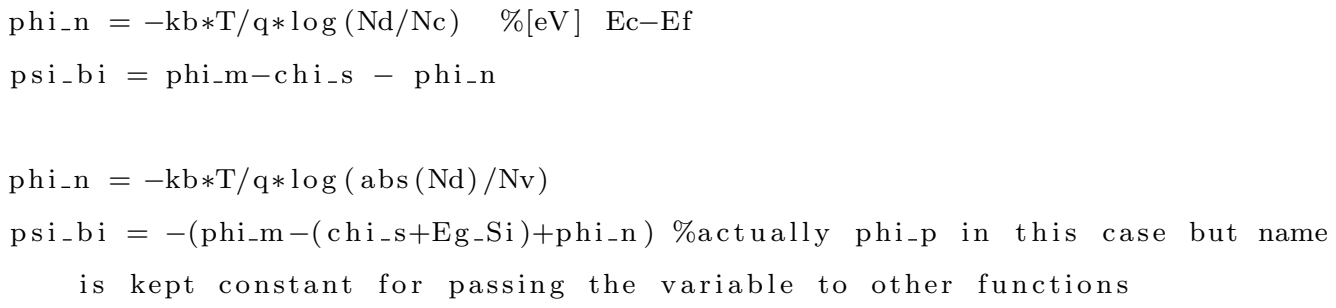

\%INITIALIZE THE SIMULATION

$[\mathrm{dx}, \mathrm{dop}, \operatorname{psi}, \mathrm{Ldi}]=$ Initialize $\left(\mathrm{Nd}, \operatorname{psi} \_\right.$bi, L_max $)$

n_max $=$ length (psi);

\%EQUILIBRIUM SOLUTION

display ('Equilibrium solution')

$[$ psi,n,p] = MIS_Equilibrium(dx, psi, psi_bi, phi_n, Ldi,Nd,dop, d_ox $)$;

\%Plot results

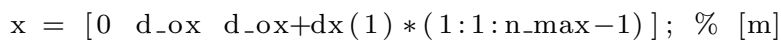

$\%$

$\%$ GENERATION RATE \%

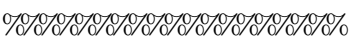

if (Generation)

\%Calculate the generation rate from the solar illumination

$\mathrm{c}=3 \mathrm{E} 8 ; \%$ speed of light $[\mathrm{cm} / \mathrm{s}]$

$\mathrm{xgen}=\mathrm{x}(3:$ end $)-\mathrm{d}_{-} \mathrm{ox}$;

load generationparam.mat;

xgenmat $=\operatorname{repmat}($ xgen, length $(\mathrm{WL}), 1) ;$

$\operatorname{delWL}=\operatorname{diff}(\mathrm{WL})$

$\operatorname{delWL}=[\operatorname{delWL}(1) ; \operatorname{delWL}]$;

Po $=(\mathrm{S} . * \text { delWL } * \text { WL } * 1 \mathrm{E}-9)^{\prime} ;$ \%convert spectrum to power. convert WL to $[\mathrm{m}]$

alpha_mat $=\operatorname{repmat}(\mathrm{a}, 1, \operatorname{length}($ xgen $)) ;$

attenuation $=\exp (-$ alpha_mat.$*$ xgenmat $)$;

Patten $=$ Po*attenuation;

Pabsorb = diff (Patten);

\%since Pabs is the power absorbed between the mesh points, calculate 


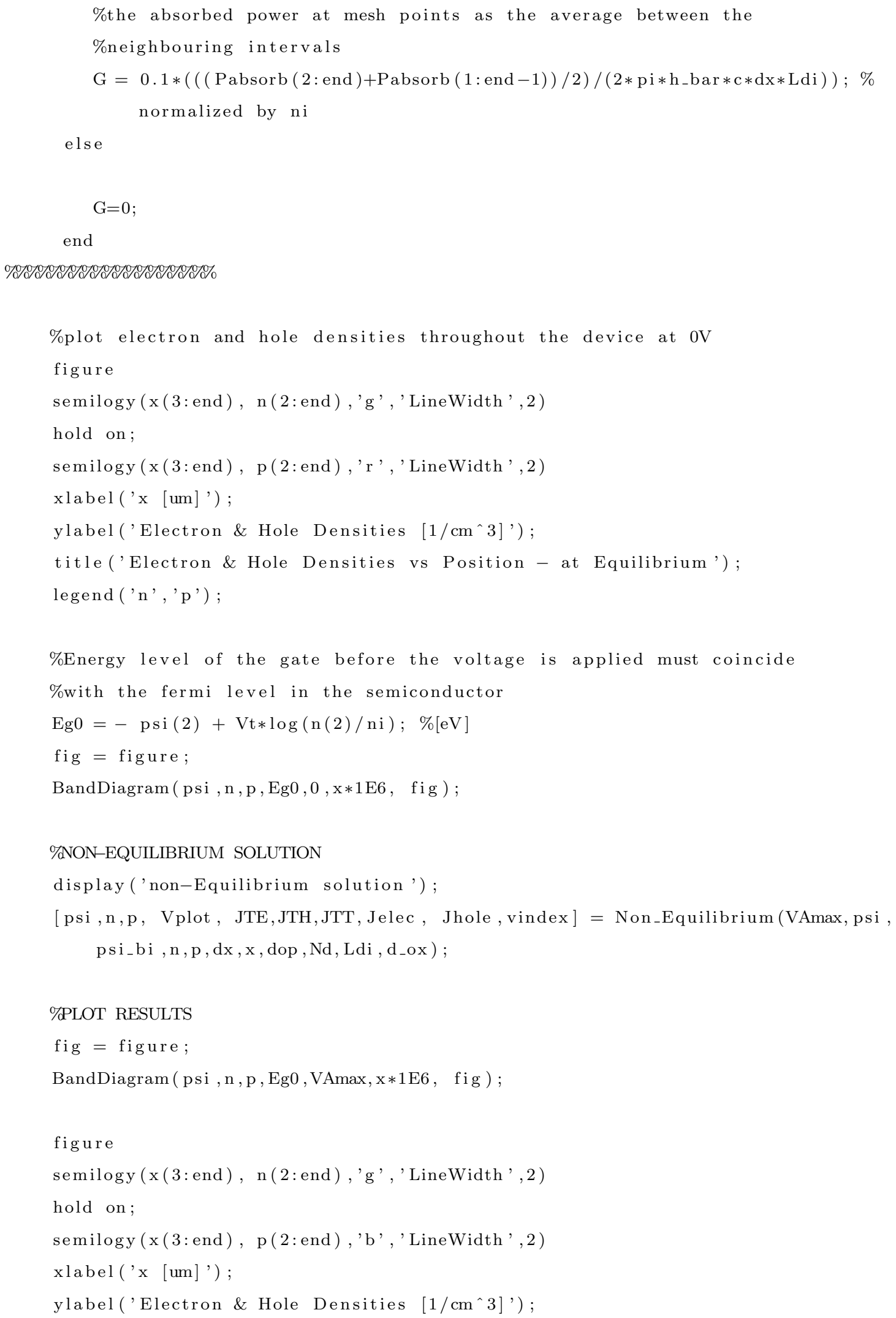




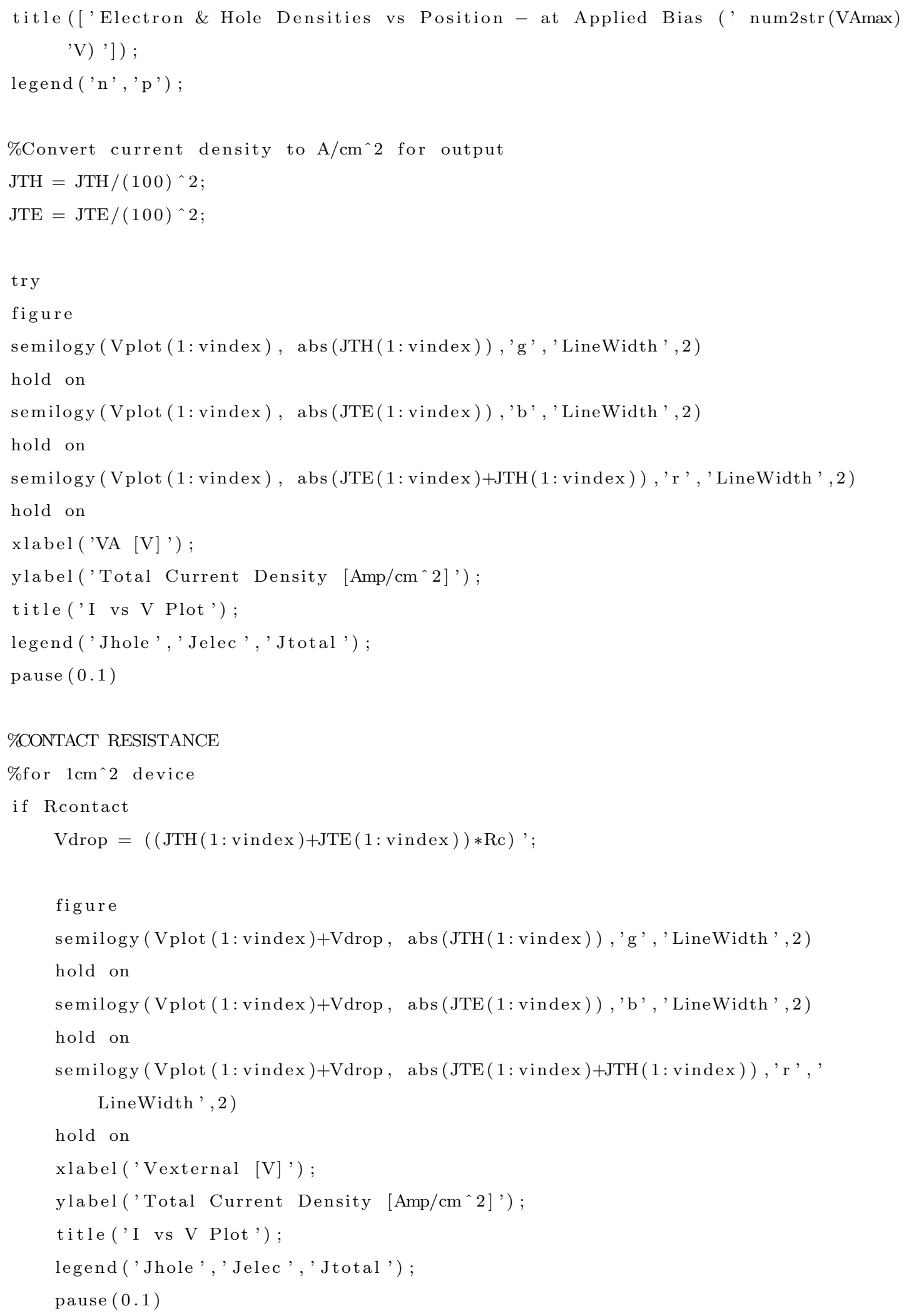




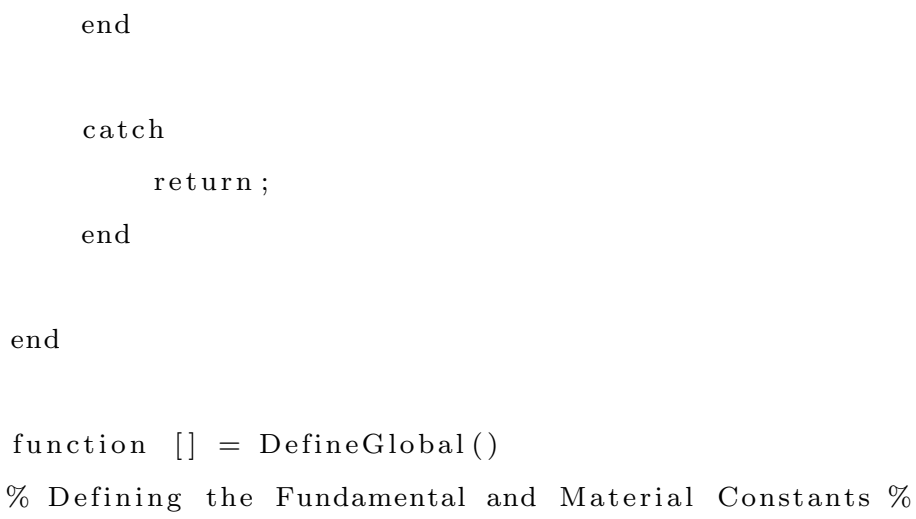




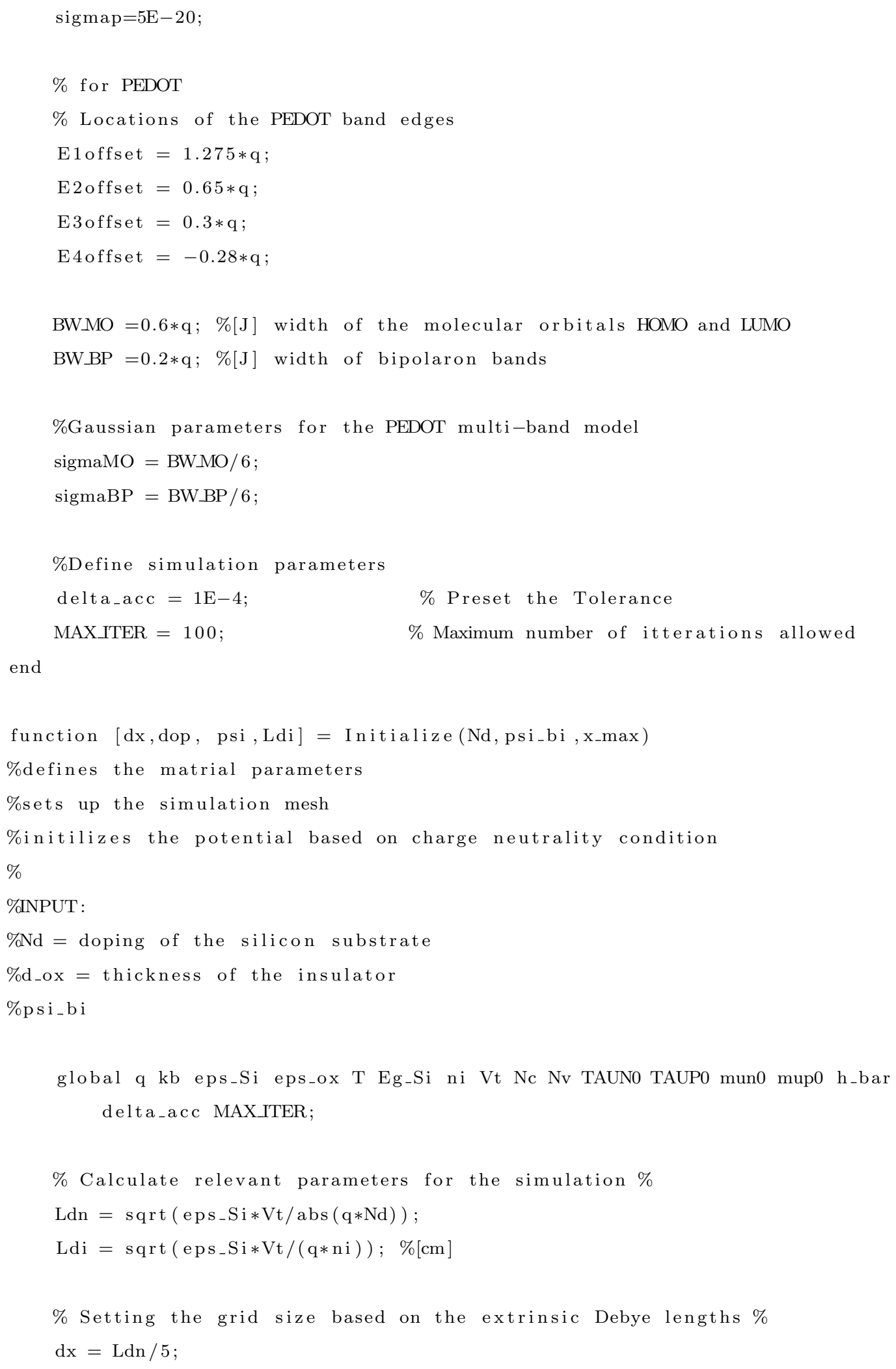


\% Calculate the required number of grid points and renormalize dx \% $\mathrm{n} \_\max =\operatorname{round}\left(\mathrm{x} \_\max / \mathrm{dx}\right)$;

\% Set up the doping $\mathrm{C}(\mathrm{x})=\mathrm{Nd}(\mathrm{x})-\mathrm{Na}(\mathrm{x})$ that is normalized with ni \% $\operatorname{dop}=\mathrm{Nd} *$ ones $\left(1, \mathrm{n}_{\_} \max \right) ;$

fid = fopen ('Ndlog.txt', ' at') ;

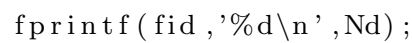

fclose (fid);

\% Initialize the potential based on the requirement of charge

$\%$ neutrality throughout the whole structure

$\mathrm{zz}=0.5 * \mathrm{dop} / \mathrm{ni} ;$

if $\mathrm{Nd}>0$ \%donor doped substrate (n-type)

$\mathrm{xx}=\mathrm{zz} *(1+\operatorname{sqrt}(1+1 \cdot /(\mathrm{zz} \cdot * \mathrm{zz}))) ;$

psi $=\mathrm{Vt} * \log (\mathrm{xx}) ; \% \% \%$ Midband potential

psiMI = psi(1)-psi_bi; \%potential at the metal/insulator interface

else \% acceptor doped substrate (p-type)

$\mathrm{xx}=\mathrm{zz} \cdot *(1-\operatorname{sqrt}(1+1 \cdot /(\mathrm{zz} \cdot * \mathrm{zz}))) ;$

psi $=\mathrm{Vt} * \log (\mathrm{xx}) ; \% \% \%$ Midband potential \%\%UMERICAL ERROR??? causes slightly complex numbers

psiMI $=$ psi $(1)+p s i \_b i ; \%$ potential at the metal/insulator interface

end

psi $=[$ psiMI psi $]$;

dop $=[0$ dop $] ; \%$ the 0 in the front is for ease in indexing so psi and dop are the same length

end

function [psi,n,p] = MIS_Equilibrium(dx, psi, psi_bi,psi_n, Ldi,Nd,dop, d_ox)

global q kb eps_Si eps_ox T Eg_Si ni Vt Nc Nv TAUNO TAUP0 mun0 mup0 h_bar delta_acc MAX_TER Dit FermiDirac SurfaceStates;

V_old $=0$

Vox $=\operatorname{psi}(2)-\operatorname{psi}(1)$;

$\operatorname{Efss}=0 ;$

$\mathrm{n}=\mathrm{ni} * \exp (\mathrm{psi} / \mathrm{Vt})$

$\mathrm{p}=\mathrm{ni} * \exp (-\mathrm{psi} / \mathrm{Vt})$; 


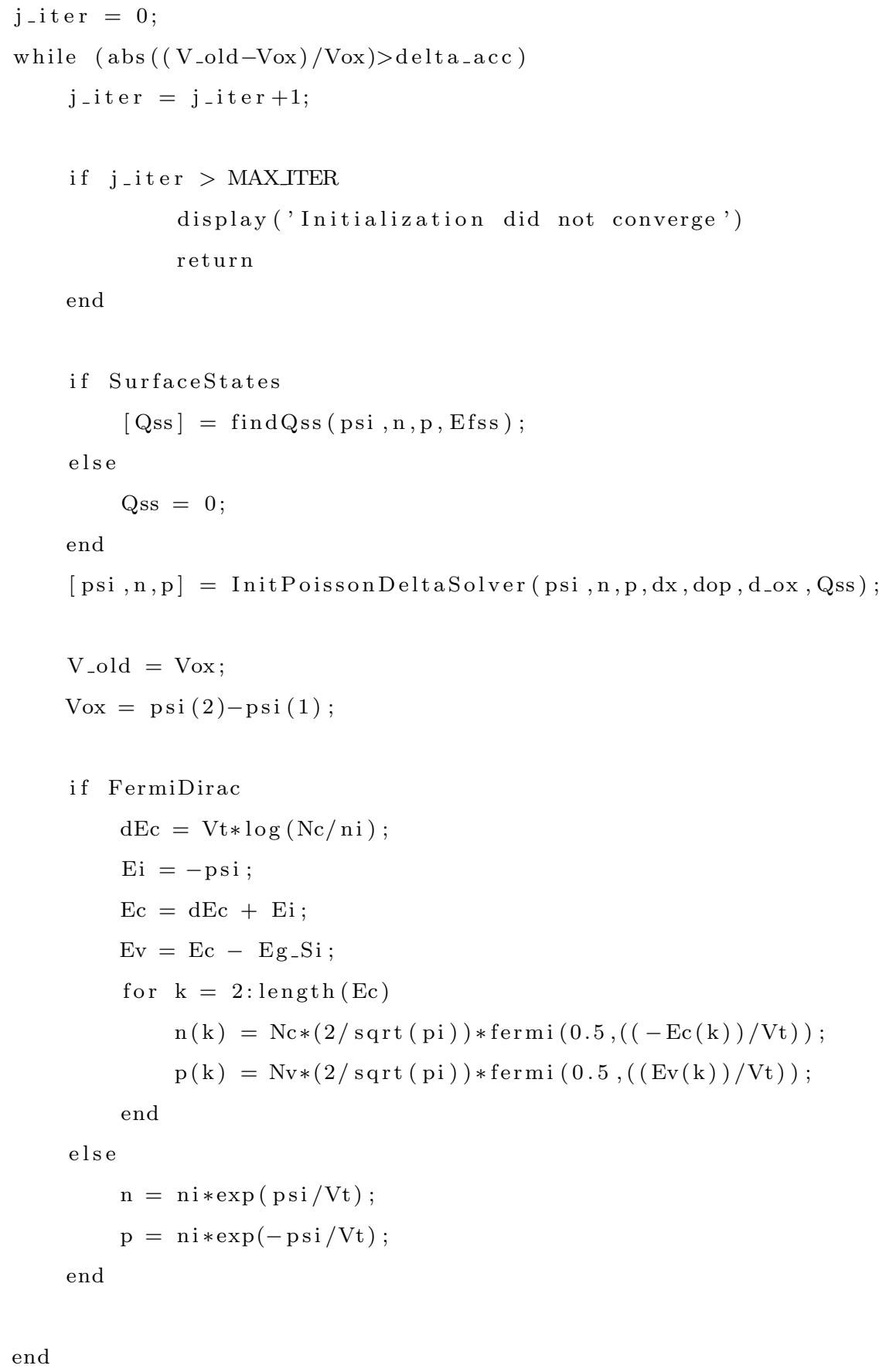


global q kb eps_Si eps_ox T Eg_Si ni Vt Nc Nv TAUNO mun0 mup0 h_bar delta_acc MAXITER Npedot FermiDirac PEDOT SurfaceStates;

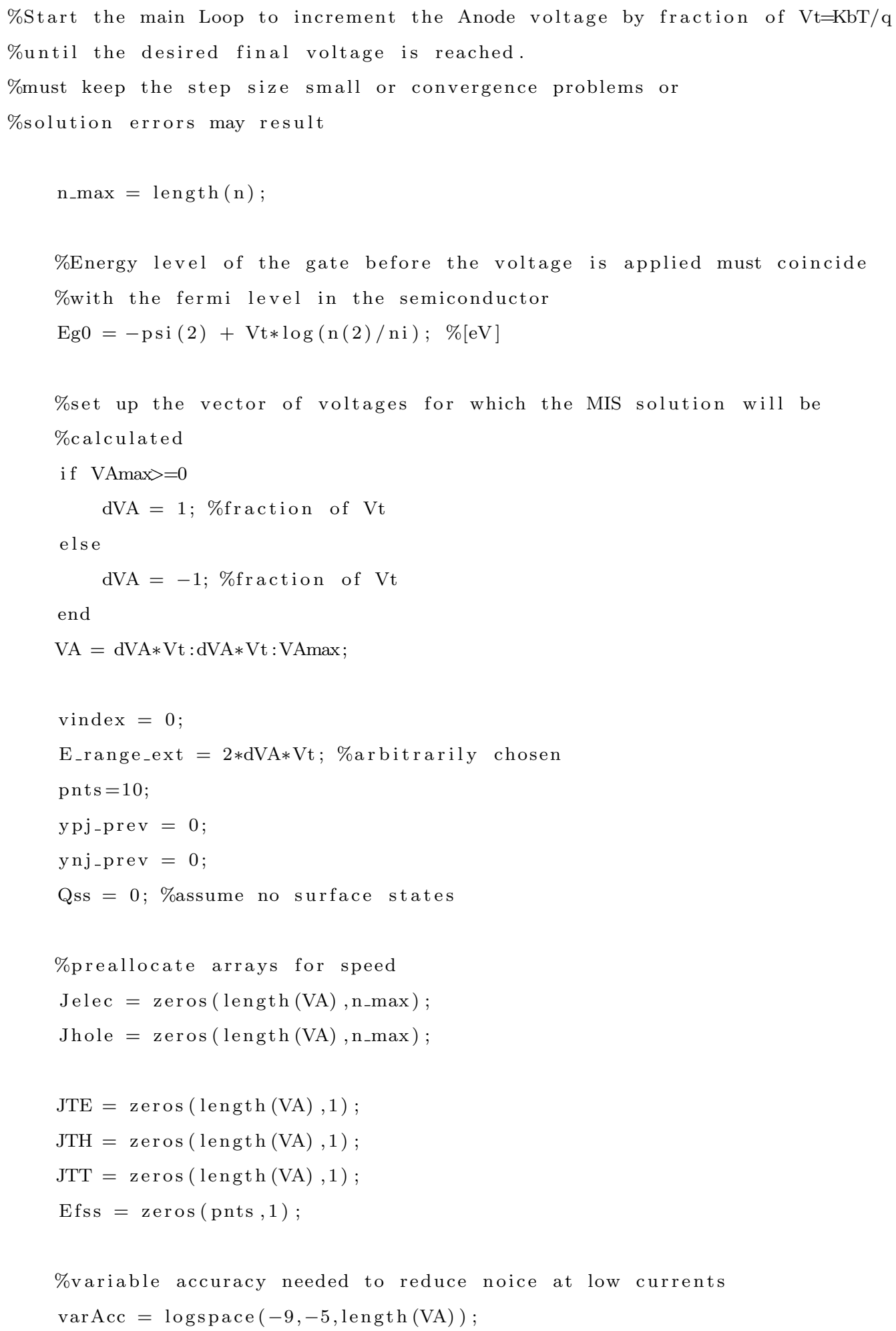


while (vindex $<$ length $(V A)$

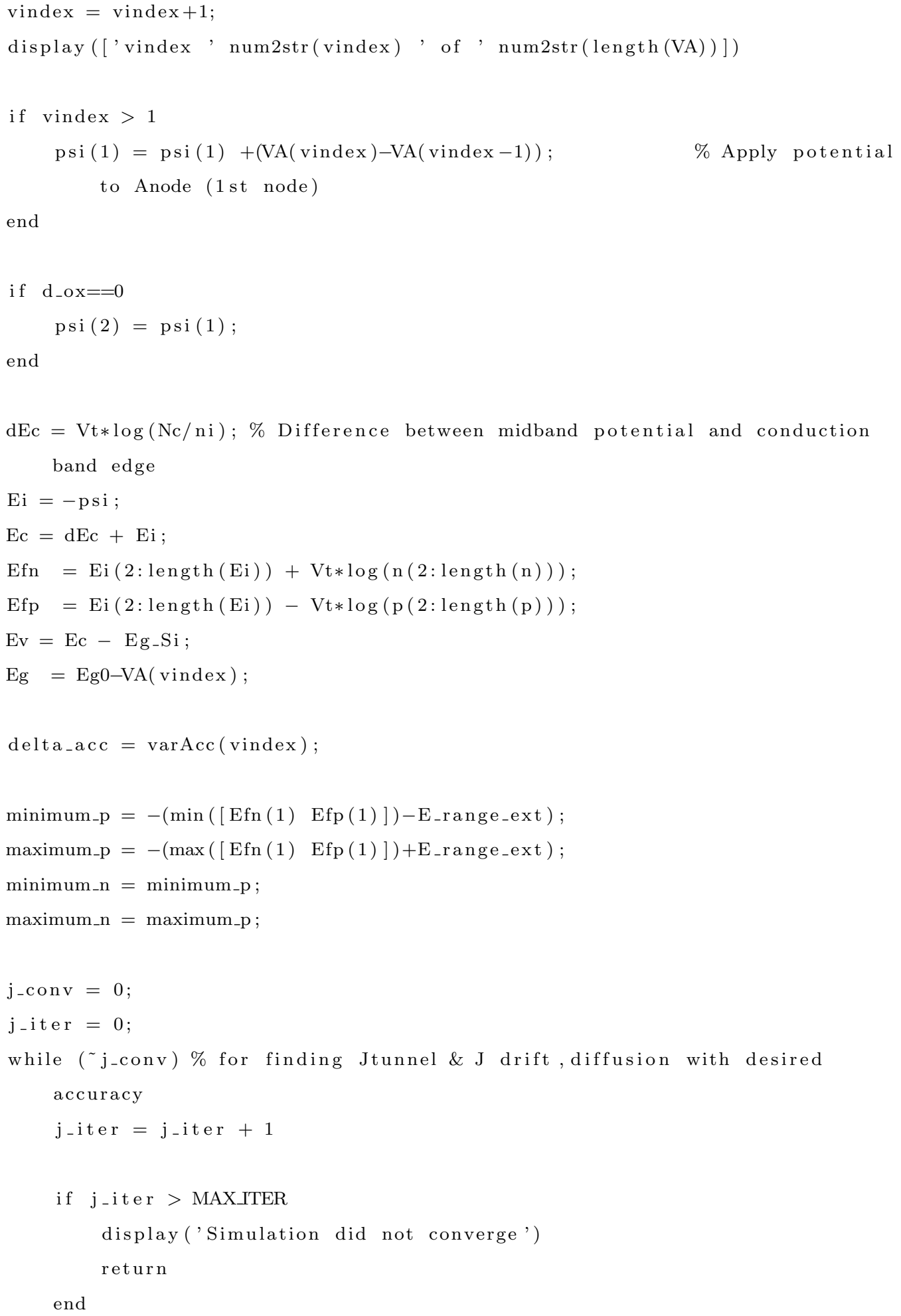




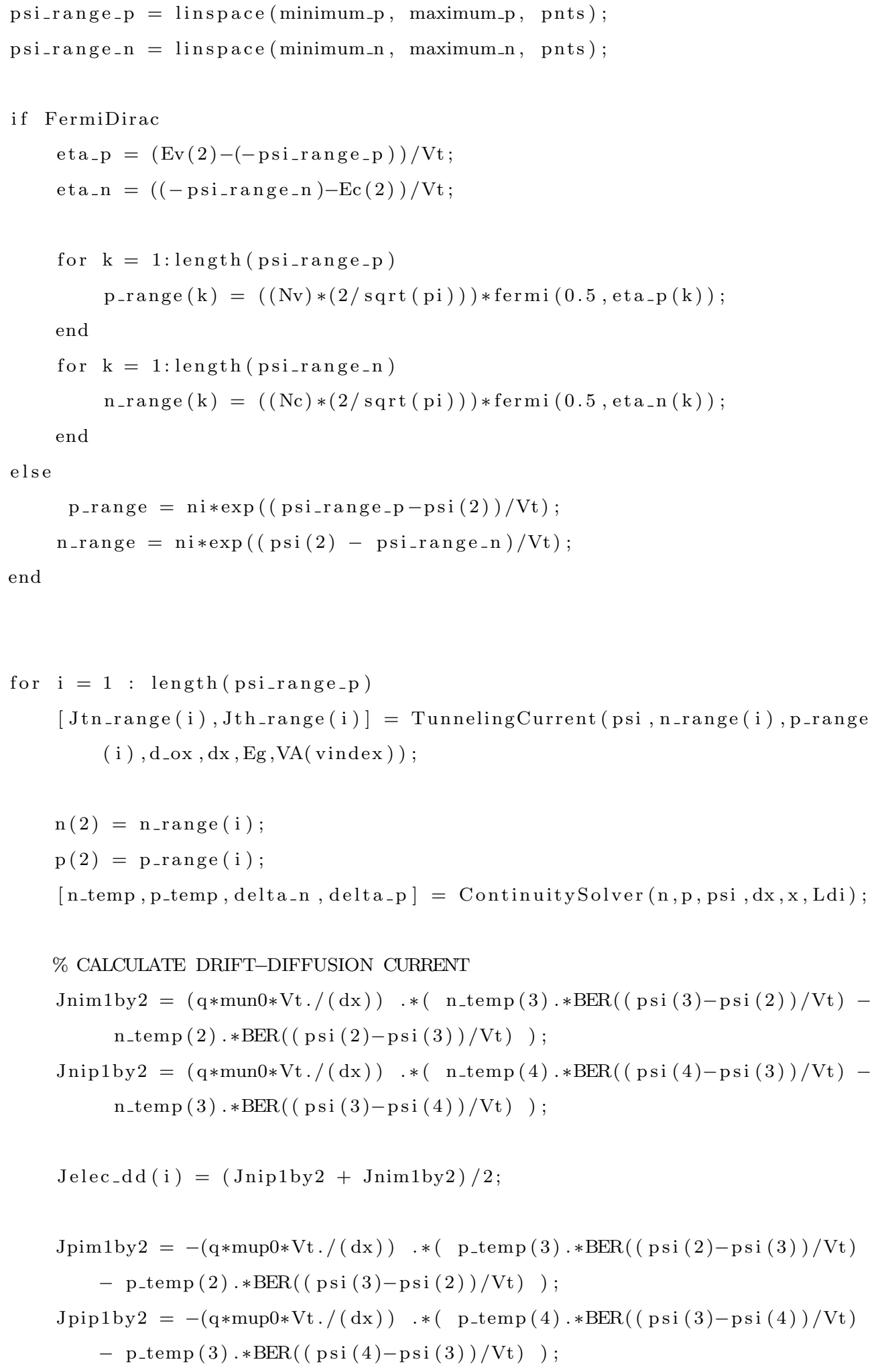


Jhole_dd $(\mathrm{i})=(\operatorname{Jpip} 1 \mathrm{by} 2+\mathrm{Jpim} 1 \mathrm{by} 2) / 2 ;$

if SurfaceStates

\%Find Fermi-level for the surface states

$\operatorname{Efss}(i)=$ findEfss $($ psi, n, p, Eg,d_ox $)$;

$[\mathrm{Jrc}, \mathrm{Jrv}]=$ findRecombinationCurrents (psi, n, p, Efss (i ));

else

$\mathrm{Jrc}=0$

$\mathrm{Jrv}=0$

end

Jtn_range $(i)=J t n \_r a n g e(i)+J r c ;$

Jth_range(i) $=$ Jth_range(i) + Jrv;

end

try

$[\operatorname{xpj}$, ypj,pindex,$\sim]=$ intersections (psi_range_p, Jth_range,psi_range_p ,

Jhole_dd , 1);

$[\mathrm{xnj}, \mathrm{ynj}, \operatorname{nindex}, \sim]=$ intersections (psi_range_n, Jtn_range,psi_range_n ,

Jelec_dd , 1);

\%OONVERGENCE AID\%

if isempty $(\mathrm{xpj})$

\%if both trends are linear, find the projected intersection

$[\mathrm{m} 1, \mathrm{~b} 1, \mathrm{rsq} 1]=$ linearRegression $\left(\mathrm{psi}_{-} \mathrm{range} \_\mathrm{p}, \mathrm{Jth} \_\mathrm{range}\right) ;$

$\left[\mathrm{m} 2, \mathrm{~b} 2, \mathrm{rsq}_{2}\right]=$ linearRegression (psi_range_p , Jhole_dd);

if $\quad \mathrm{rsq} 1>0.9 \& \mathrm{rsq} 2>0.9$

$\operatorname{xpj}=(\mathrm{b} 2-\mathrm{b} 1) /(\mathrm{m} 1-\mathrm{m} 2) ;$

$\mathrm{ypj}=\mathrm{m} 1 * \mathrm{xpj}+\mathrm{b} 1$;

xp_min $=x p j-$ E_range_ext;

xp_max $=x p j+E_{-}$range_ext;

else

err = MException('ConvergeChk: NoInterceptFound', , ['no intercept found for Efp']');

throw (err);

end

else

$\operatorname{xpj}=\operatorname{xpj}(1) ;$ 


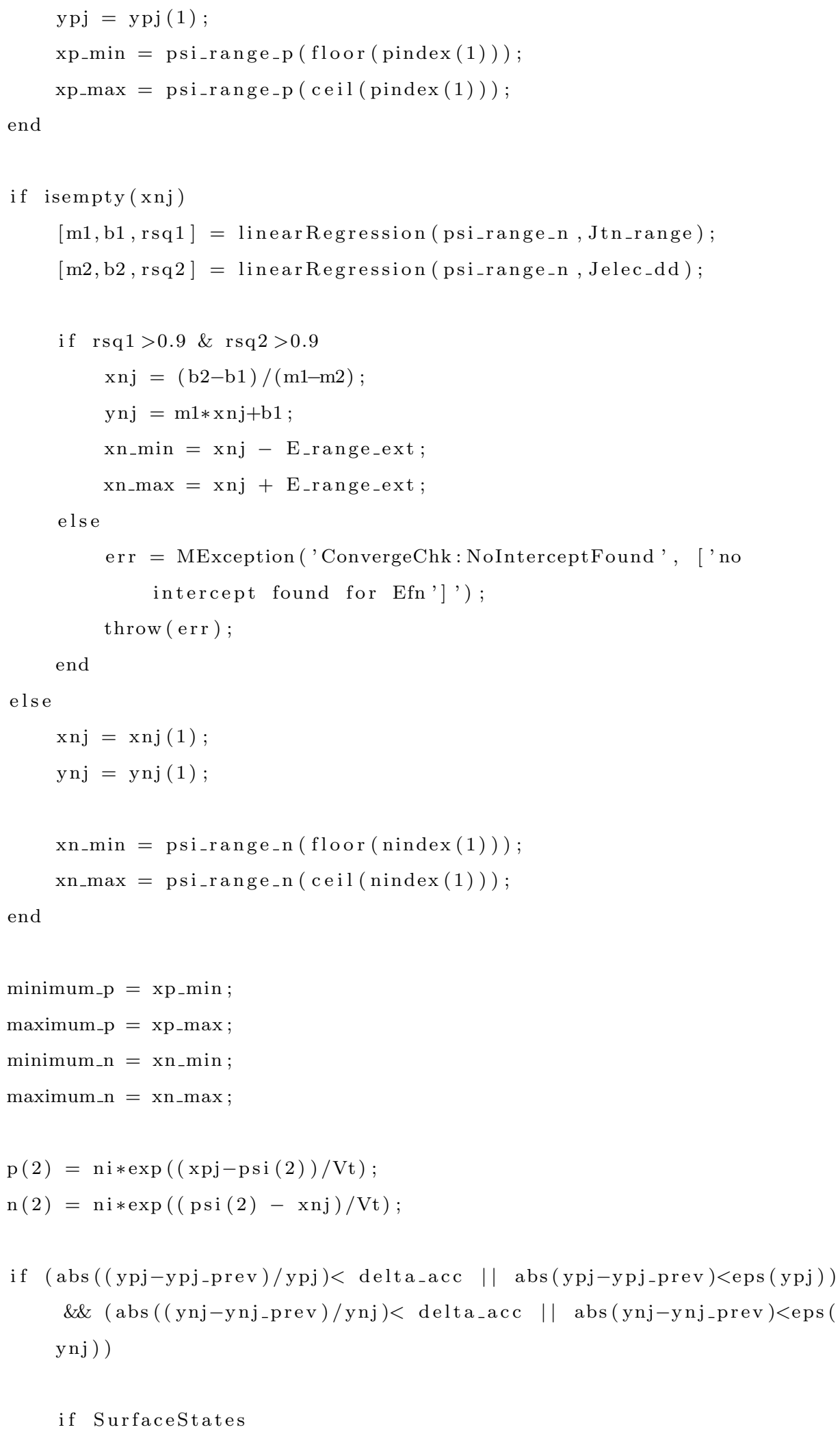




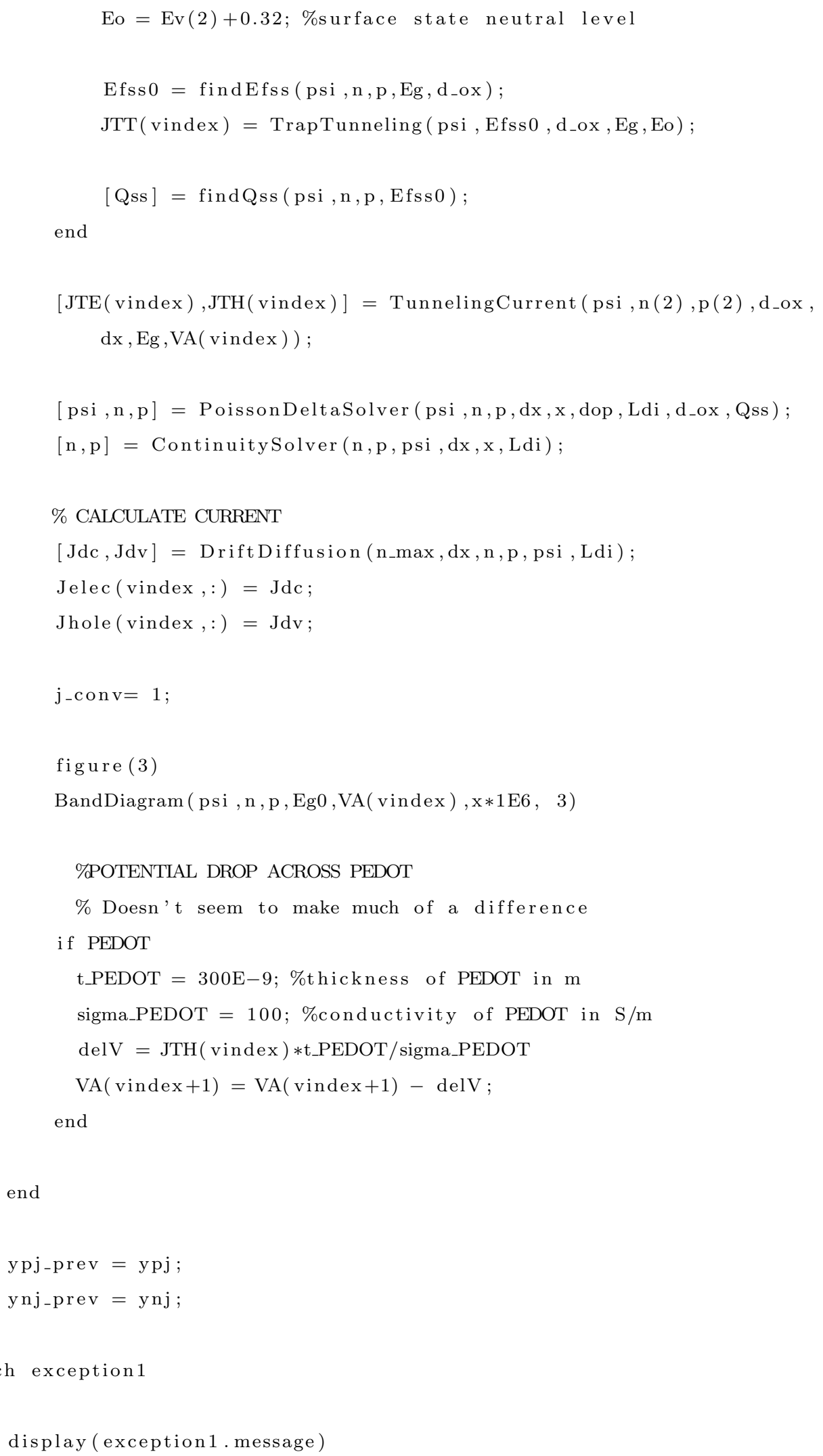


global q kb eps_Si eps_ox T Eg_Si ni Vt Nc Nv TAUNO TAUP0 mun0 mup0 h_bar delta_acc MAX_TER Ec_offset Ev_offset SiBarrier PEDOT Npedot E1offset E2offset E3offset E4offset BWMO BW_BP sigmaMO sigmaBP;

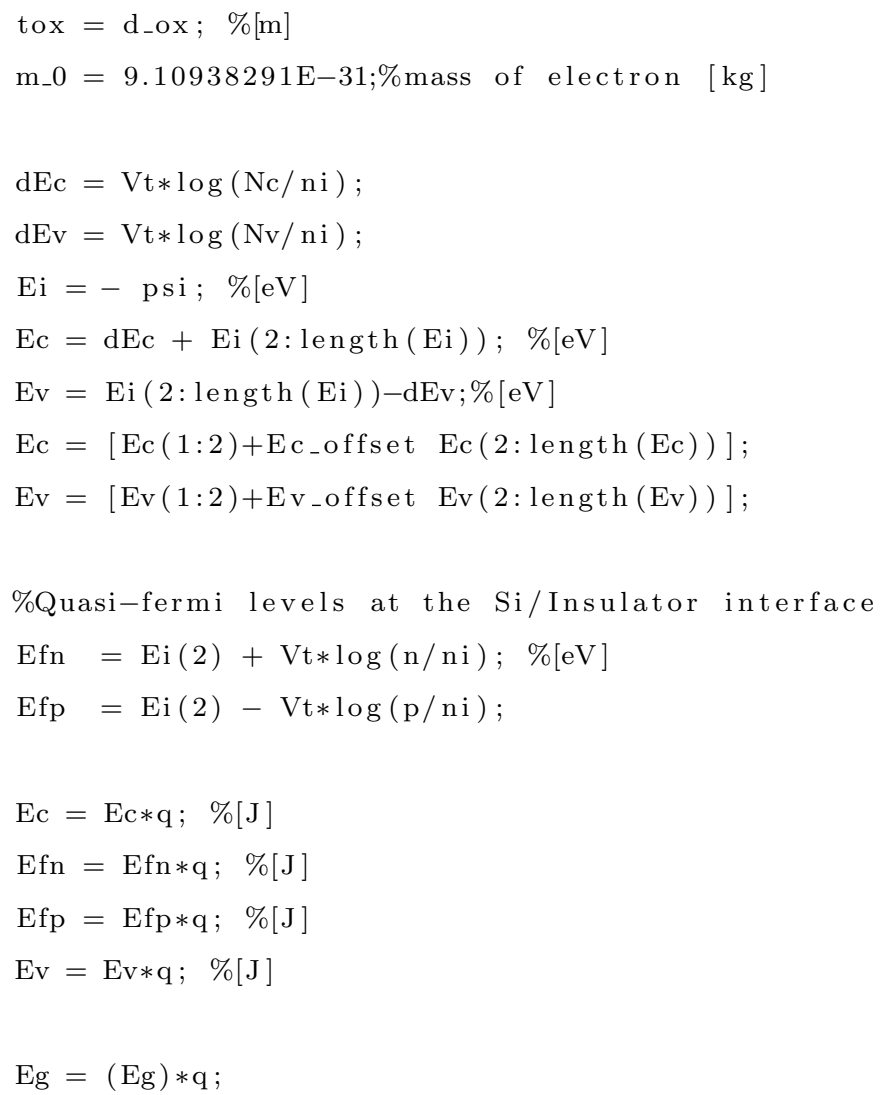




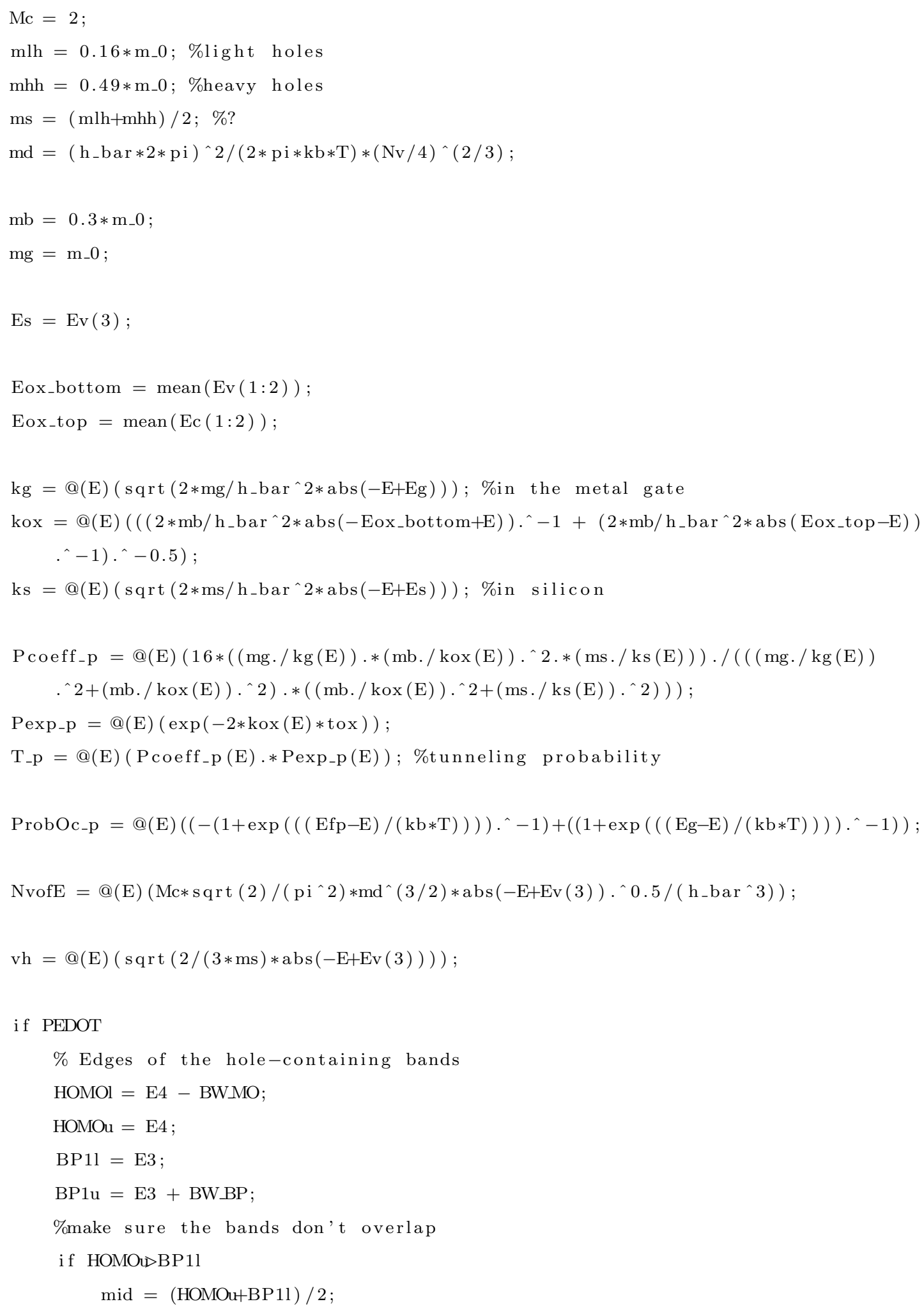




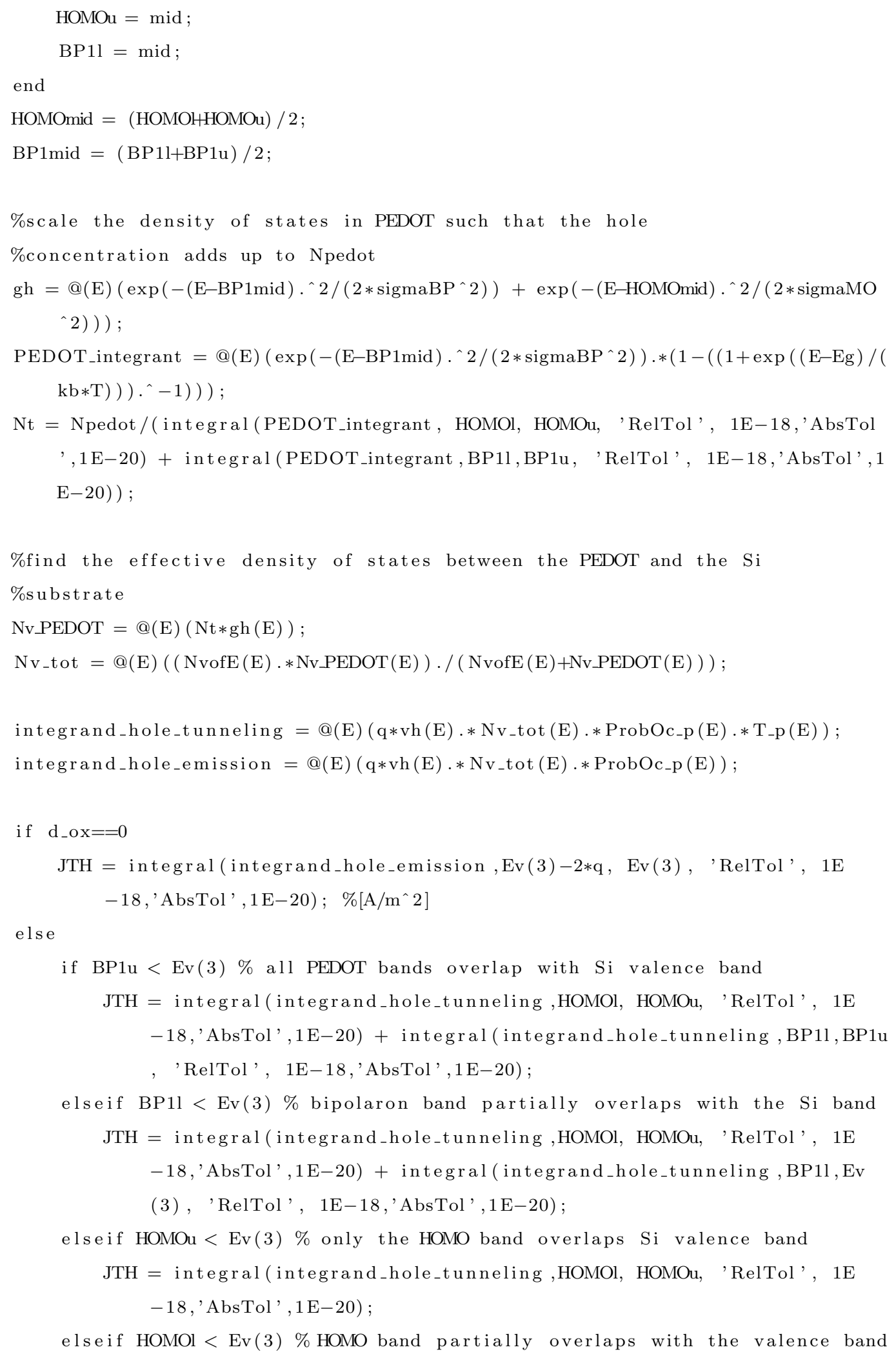




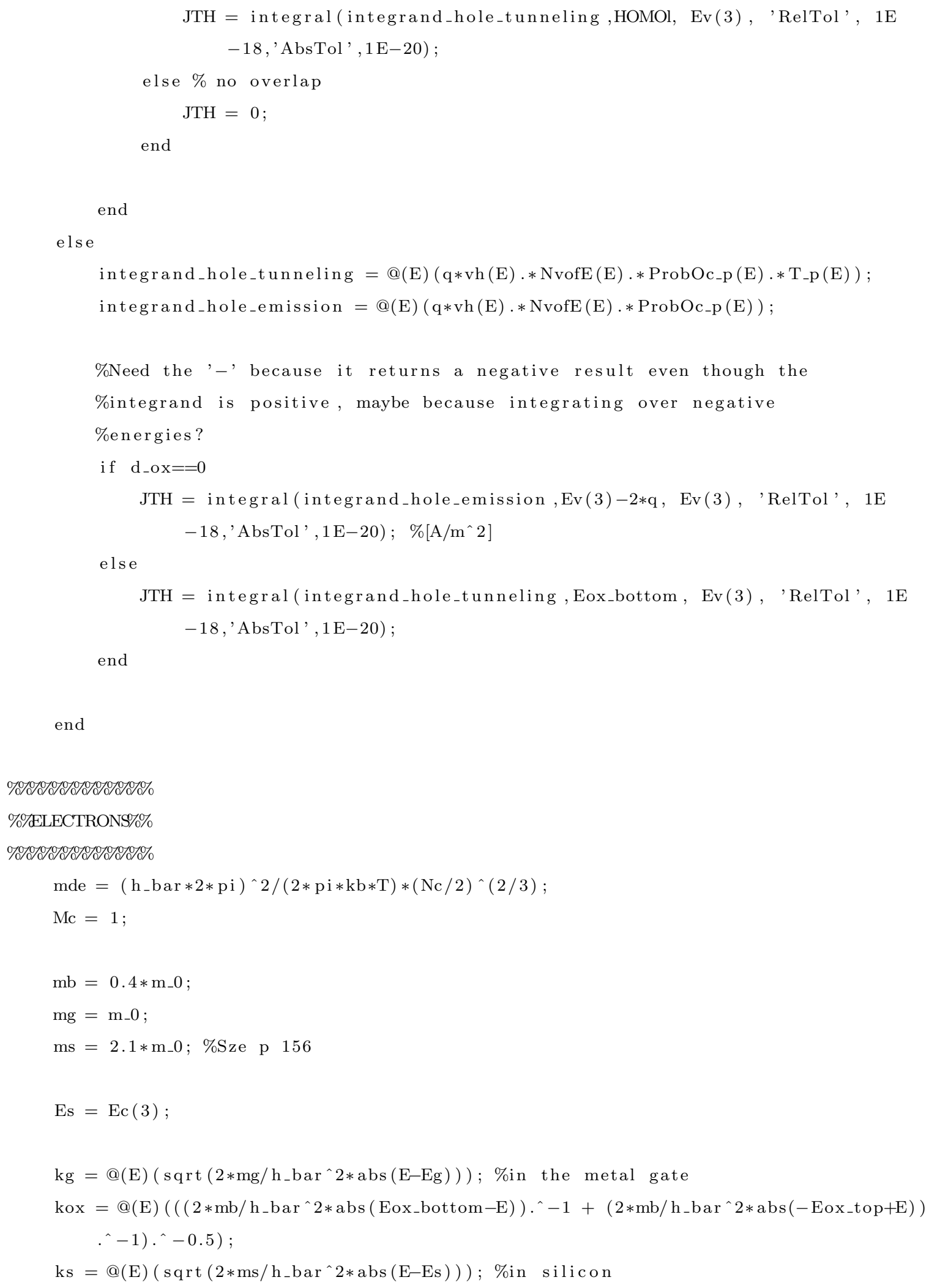




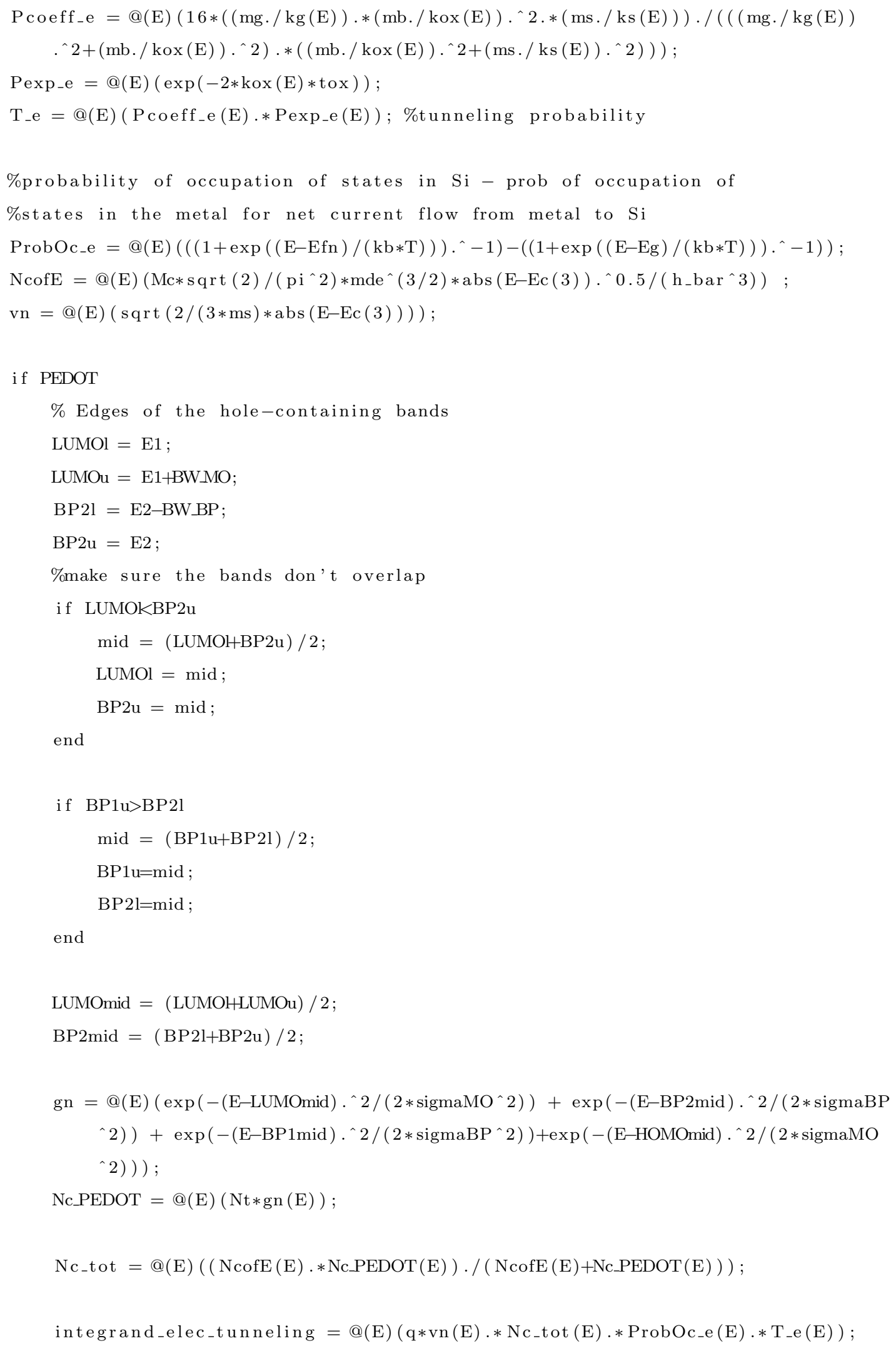




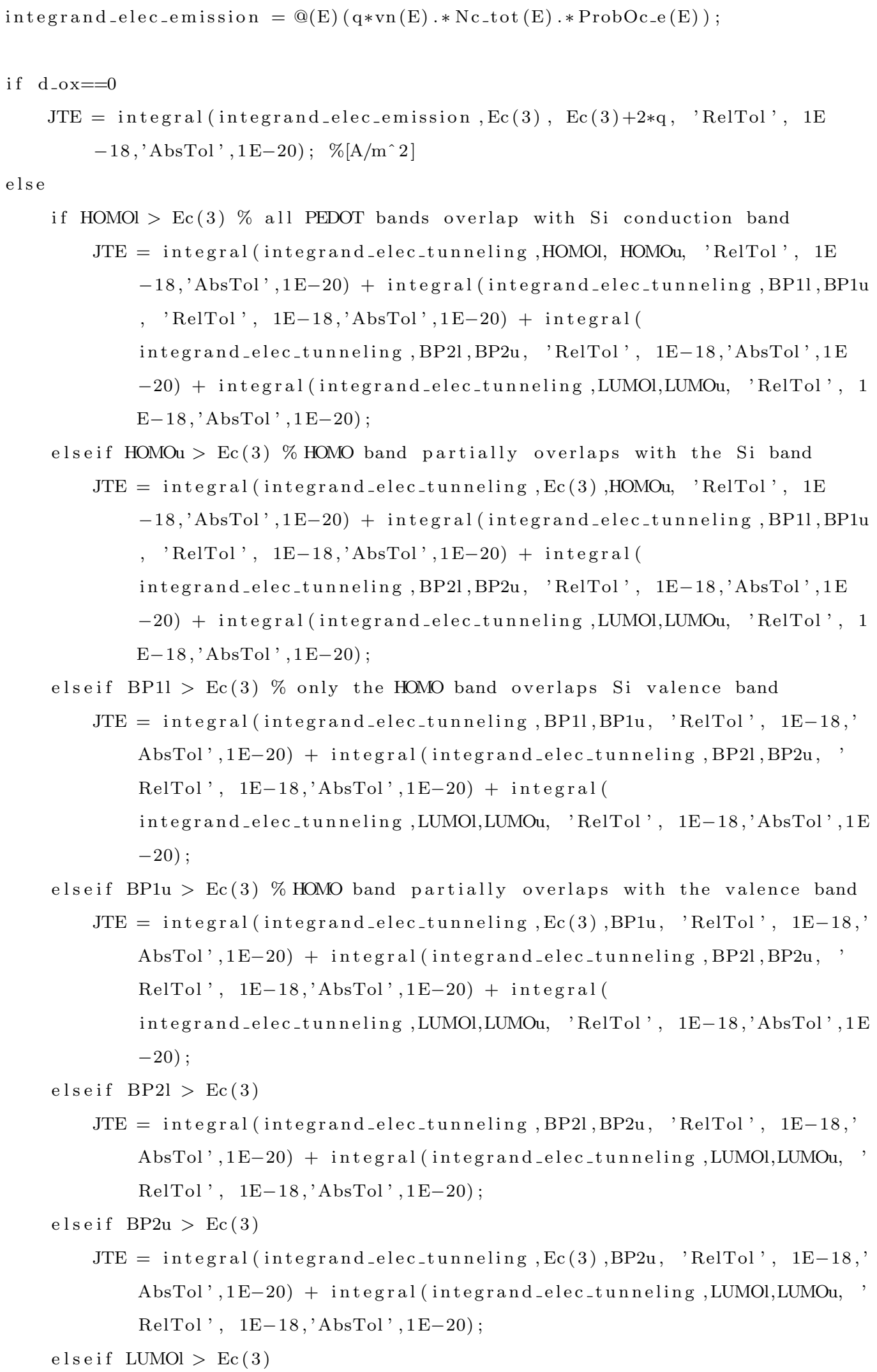




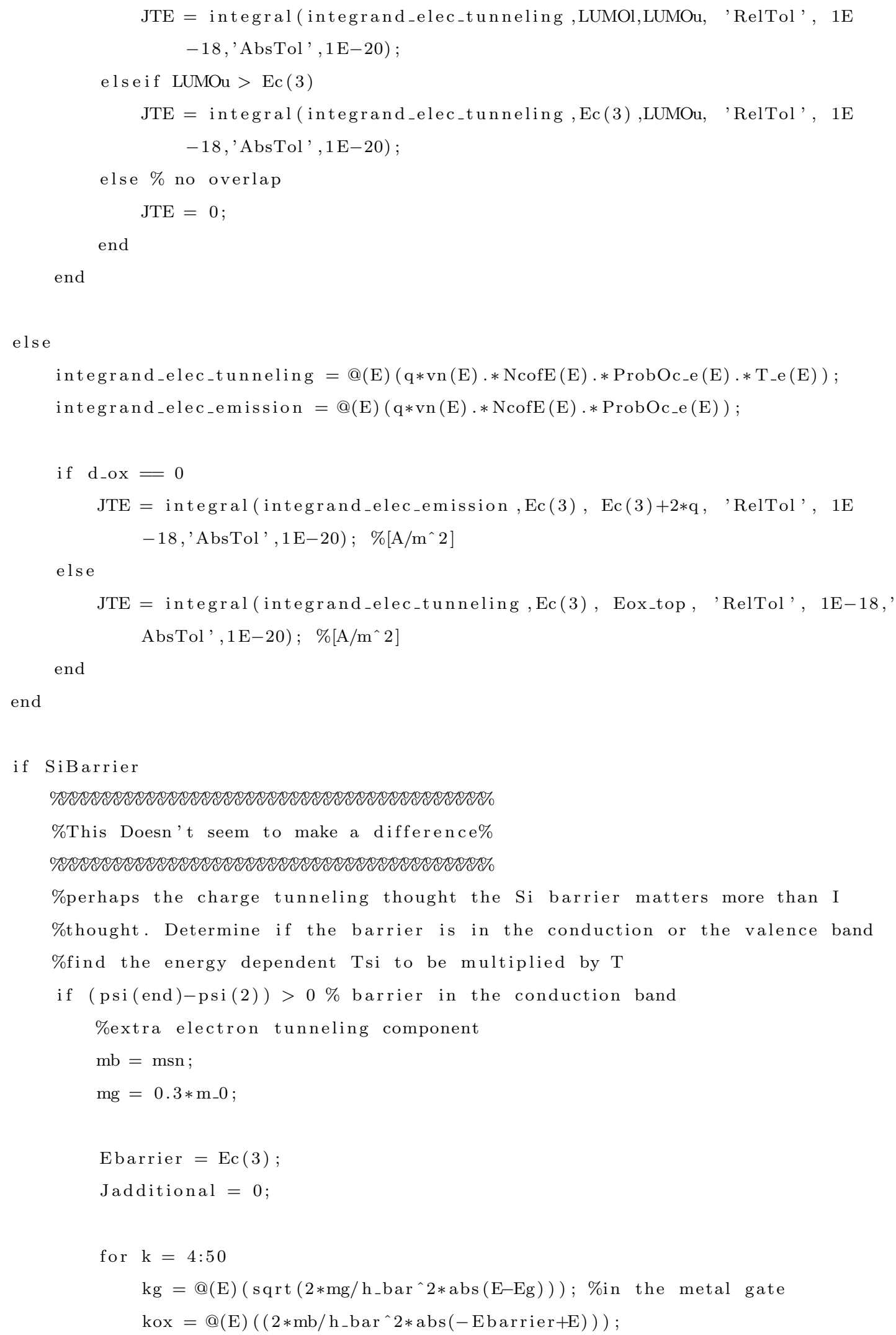




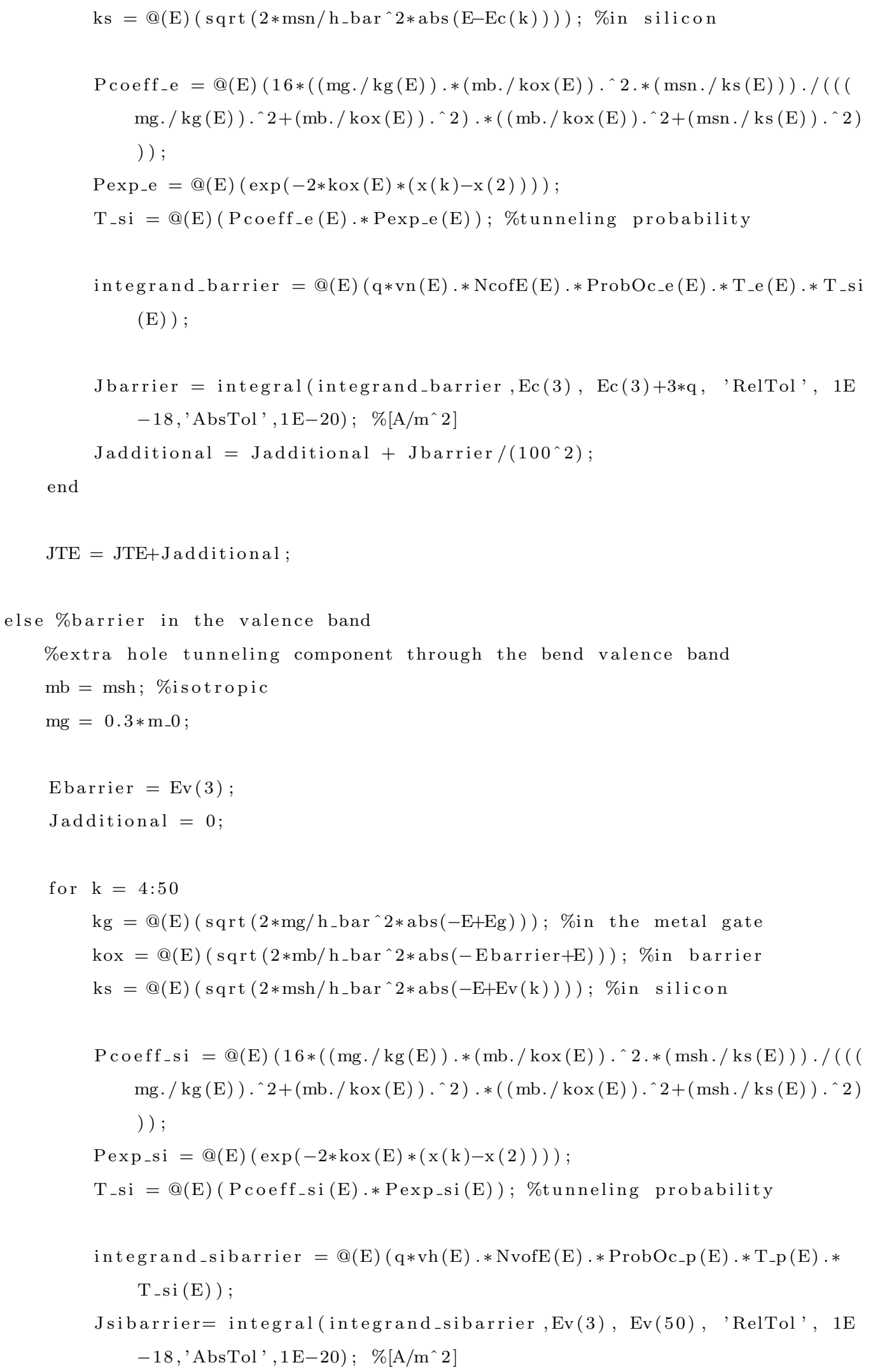


$\mathrm{Jadditional}=\mathrm{Jadditional}+\mathrm{J}$ sibarrier $; \%\left[\mathrm{~A} / \mathrm{cm}^{\wedge} 2\right]$

end

$\mathrm{JTH}=\mathrm{JTH}+\mathrm{Jadditional} ;$

end

end

end

function [psi, n,p, delta] = PoissonDeltaSolver $(\mathrm{psi}, \mathrm{n}, \mathrm{p}, \mathrm{dx}, \mathrm{x}, \mathrm{dop}$, Ldi, d_ox, Qss $)$

global q kb eps_Si eps_ox T Eg_Si ni Vt Nc Nv TAUN0 TAUP0 mun0 mup0 h_bar delta_acc MAX_ITER eps_o Dit;

\%Uses Boltzman statistics

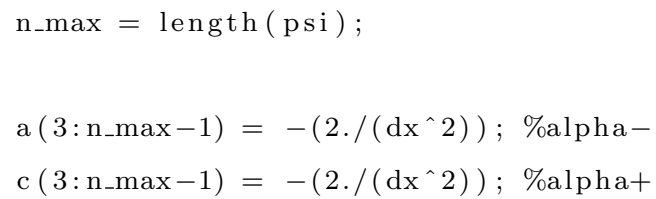




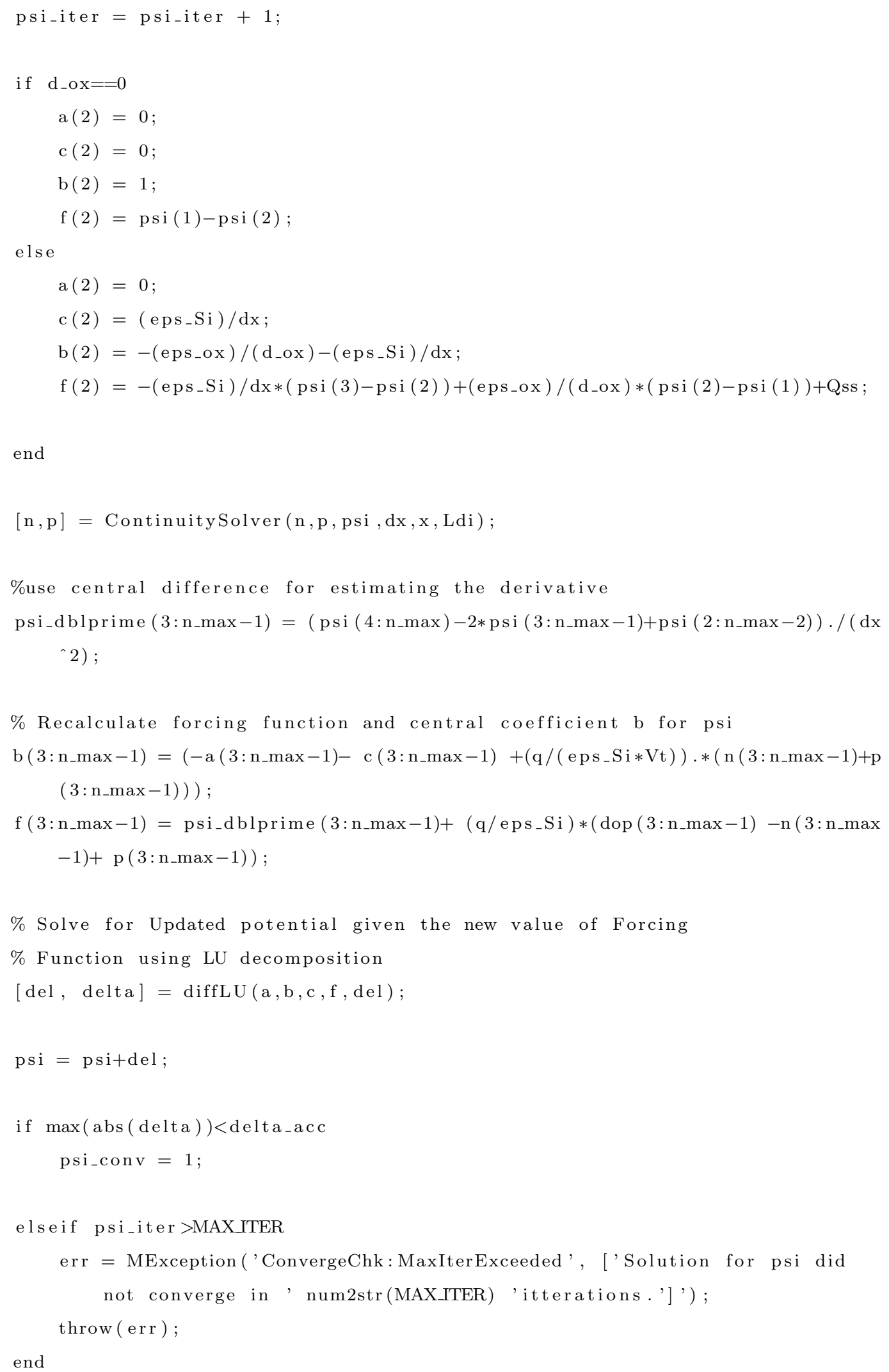


end

function [psi,n,p,delta] = InitPoissonDeltaSolver(psi,n,p,dx,dop,d_ox, Qss)

\%Uses Boltzman statistics

global q kb eps_Si eps_ox T Eg_Si ni Vt Nc Nv TAUNO TAUP0 mun0 mup0 h_bar delta_acc MAX_ITER eps_o Dit;

n_max $=$ length $($ psi $) ;$

$\mathrm{a}\left(3: \mathrm{n} \_\max -1\right)=-\left(2 . /\left(\mathrm{dx}^{\wedge} 2\right)\right) ; \%$ alpha -

$\mathrm{c}\left(3: \mathrm{n}_{-} \max -1\right)=-\left(2 . /\left(\mathrm{dx}^{\wedge} 2\right)\right) ; \%$ alpha+

$\%$ (B) Define the elements of the coefficient matrix and initialize the forcing $\%$ function at the ohmic contacts

$\mathrm{a}(1)=0$;

$c(1)=0$;

$\mathrm{b}(1)=1 ;$

$\mathrm{f}(1)=0$;

$\mathrm{a}\left(\mathrm{n} \_\max \right)=0$;

$\mathrm{c}\left(\mathrm{n} \_\max \right)=0$;

$\mathrm{b}\left(\mathrm{n} \_\max \right)=1$;

$\mathrm{f}\left(\mathrm{n} \_\max \right)=0$;

$\operatorname{del}=\operatorname{zeros}(\operatorname{size}(\operatorname{psi}))$

psi_conv $=0$;

psi_iter $=0$;

while ( psi_conv)

psi_iter $=$ psi_iter +1 ;

if $d_{-}-\mathrm{x}==0$

$$
\begin{aligned}
& \mathrm{a}(2)=0 ; \\
& \mathrm{c}(2)=0 ; \\
& \mathrm{b}(2)=1 ;
\end{aligned}
$$




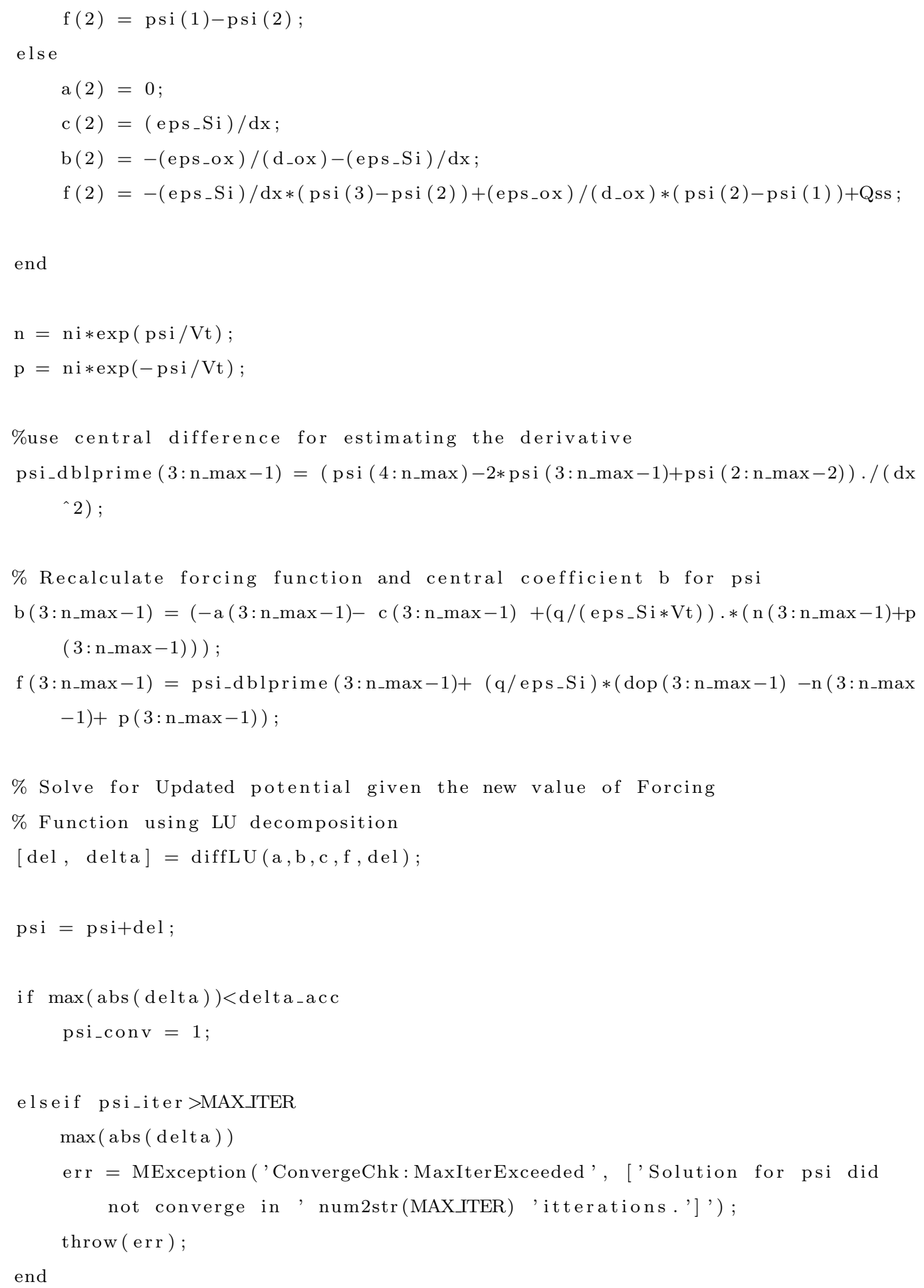


function [n,p, deltan, deltap $]=$ ContinuitySolver (n,p, psi, dx, $x$, Ldi)

global q kb eps_Si eps_ox T Eg_Si ni Vt Nc Nv TAUNO TAUP0 mun0 mup0 h_bar delta_acc MAXITER G SRH;

\% Solve Continuity Equation for Electron and Holes using LU Decomposition

\% Uses Scharfetter-Gummel discretization

\% Based on "1D Drift Diffusion simulator for modeling pn-junction Diode" by

\% Pinakpani Nayak with typos and errors fixed

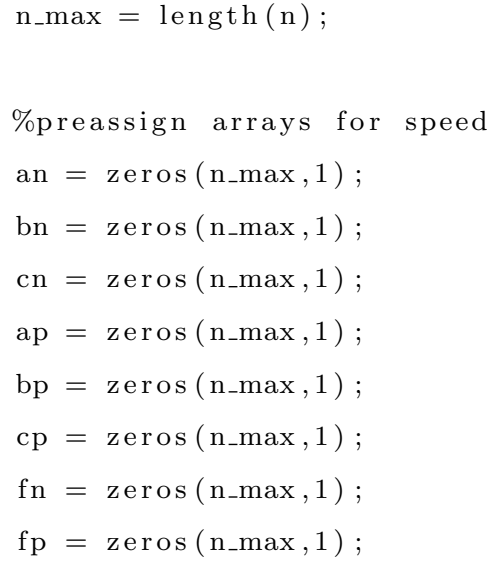

\%(A) Define the elements of the coefficient matrix and initialize the forcing $\%$ function at the ohmic contacts for ELECTRON and HOLE Continuity Eqns

$\%$ The first element in $n$ and $p$ arrays doesn't matter, leave it the same as $\%$ it was
$\operatorname{an}(1)=0$
$\% \mathrm{Co}-$ ef for electron at Anode
$\operatorname{bn}(1)=1$;
\%Co-ef for electron at Anode
$\operatorname{cn}(1)=0$;
$\%$ Co-ef for electron at Anode
$\operatorname{ap}(1)=0$
$\% \mathrm{Co}-$ ef for hole
at Anode
$\mathrm{bp}(1)=1$;
$\% \mathrm{Co}-$ ef for hole
at Anode
$\operatorname{cp}(1)=0$;
$\% \mathrm{Co}-$ ef for hole
at Anode
$\mathrm{fn}(1)=\mathrm{n}(1)$;
$\mathrm{fp}(1)=\mathrm{p}(1)$;
an $(2)=0 ;$
$\%$ Co-ef for electron at Anode
$\operatorname{bn}(2)=1$;
$\%$ Co-ef for electron at Anode
$\operatorname{cn}(2)=0$;
$\% \mathrm{Co}-$ ef for electron at Anode
$\operatorname{ap}(2)=0 ;$
$\% \mathrm{Co}-$ ef for hole at Anode 


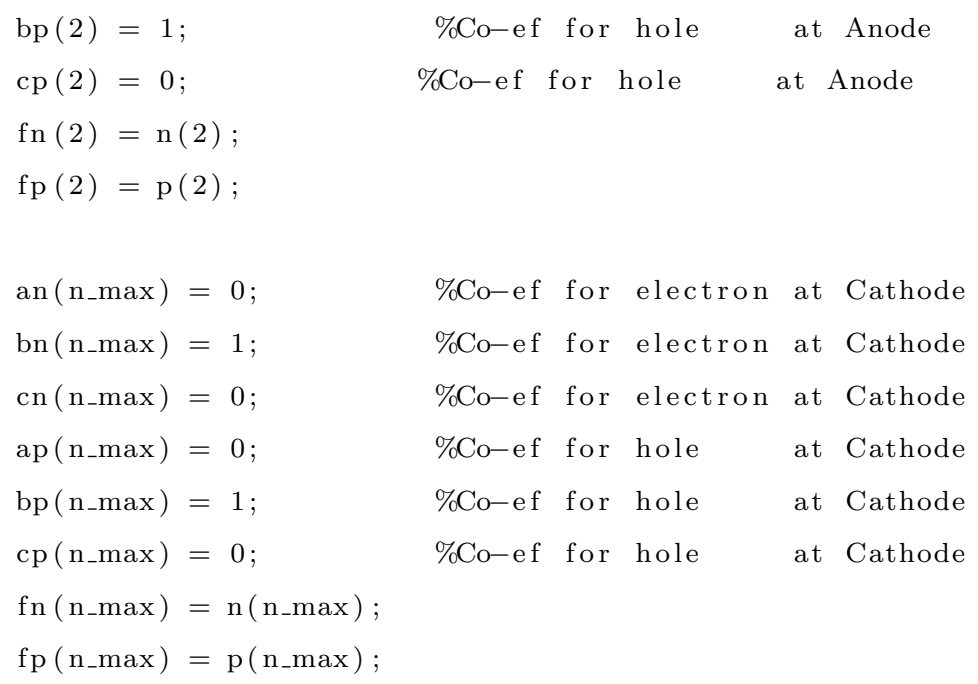

$\%$ Forcing Function for ELECTRON and HOLE Continuity eqns

fn $\left(3: n_{-} \max -1\right)=(-\mathrm{ni}) \cdot / \mathrm{D}+\mathrm{G}$;

fp $(3:$ n_max -1$)=(-\mathrm{ni}) \cdot / \mathrm{D}+\mathrm{G}$;

else 


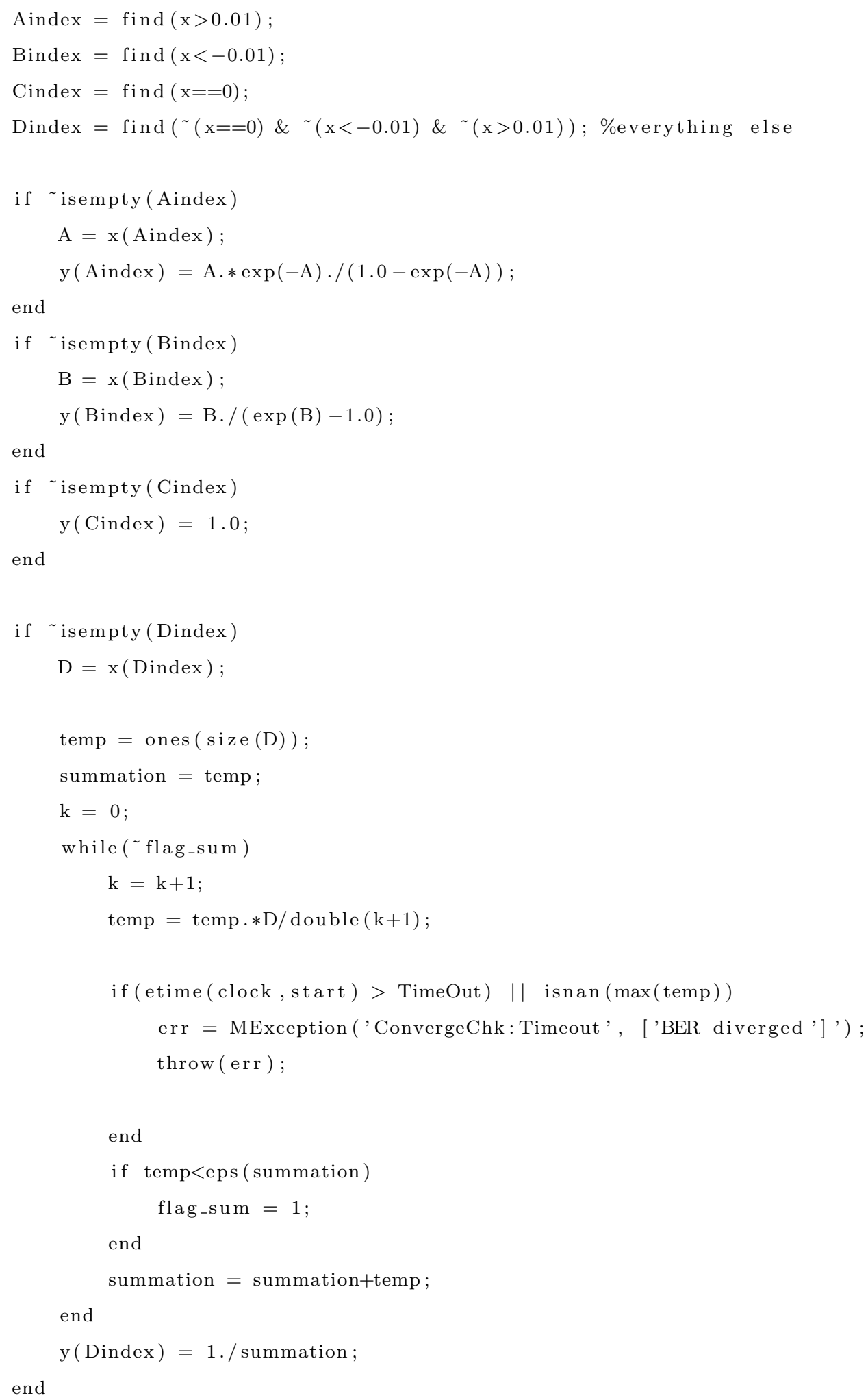


global q kb eps_Si eps_ox T Eg_Si ni Vt Nc Nv TAUNO TAUP0 mun0 mup0 h_bar delta_acc MAXITER Ec_offset Ev_offset;

$\mathrm{dEc}=\mathrm{Vt} * \log (\mathrm{Nc} / \mathrm{ni}) ; \%$ Difference between midband potential and conduction band edge

Ei $=-p s i$

$\mathrm{Ec}=\mathrm{dEc}+\mathrm{Ei}$

$\operatorname{Efn}=\operatorname{Ei}(2: \operatorname{length}(\mathrm{Ei}))+\mathrm{Vt} * \log (\mathrm{n}(2:$ end $) / \mathrm{ni}) ;$

$\operatorname{Efp}=\operatorname{Ei}(2: \operatorname{length}(\mathrm{Ei}))-\mathrm{Vt} * \log (\mathrm{p}(2:$ end $) / \mathrm{ni}) ;$

$\mathrm{Ev}=\mathrm{Ec}-\mathrm{Eg}_{-} \mathrm{Si}$

end

function [] = BandDiagram (psi,n,p,Eg0,Va, x, fig )

\%plot the band diagram

global q kb eps_Si eps_ox T Eg_Si ni Vt Nc Nv TAUNO TAUP0 mun0 mup0 h_bar delta_acc MAXITER Ec_offset Ev_offset;

$[\mathrm{Ei}, \mathrm{Ec}, \mathrm{Efn}, \mathrm{Efp}, \mathrm{Ev}]=\operatorname{psitoE}(\mathrm{psi}, \mathrm{n}, \mathrm{p}) ;$

$\mathrm{Eg}=\mathrm{Eg} 0-\mathrm{Va}$

$\mathrm{xmin}=-0.2 ;$

$\operatorname{xmax}=5 ;$

$\mathrm{ymin}=-2$;

$\operatorname{ymax}=1 ;$

xgate $=$ linspace $(-0.1 * \max (\mathrm{x}), 0,2) ;$

$\%$ for display purposes only just to make the oxide layer visible

$\mathrm{x}(2$ : end $)=\mathrm{x}(2$ : end $)-\mathrm{x}(2) ;$

$\mathrm{x}(2)=\operatorname{xmax} * 0.1$

$\mathrm{x}(3$ : end $)=\mathrm{x}(3$ : end $)+\mathrm{x}(2) ;$

figure (fig)

$\operatorname{plot}([\mathrm{x}(1: 2) \mathrm{x}(2:$ length (Ec) ) $], \quad[$ Ec $(1: 2)+$ Ec_offset Ec(2:length(Ec)) ], 'black', , LineWidth',2); \% for $\mathrm{SiO} 2$ insulator

hold on;

plot $\left([\mathrm{x}(1: 2) \mathrm{x}(2:\right.$ length $(\mathrm{Ev}))], \quad\left[\mathrm{Ev}(1: 2)+\mathrm{Ev}_{\text {_offset }} \operatorname{Ev}(2:\right.$ length (Ev)) ], 'black , ,' LineWidth', 2); \% for SiO2 insulator

hold on;

plot (x (1: length (Ei)), Ei (1: length (Ei)),'--black', 'LineWidth', 2) ;

hold on; 


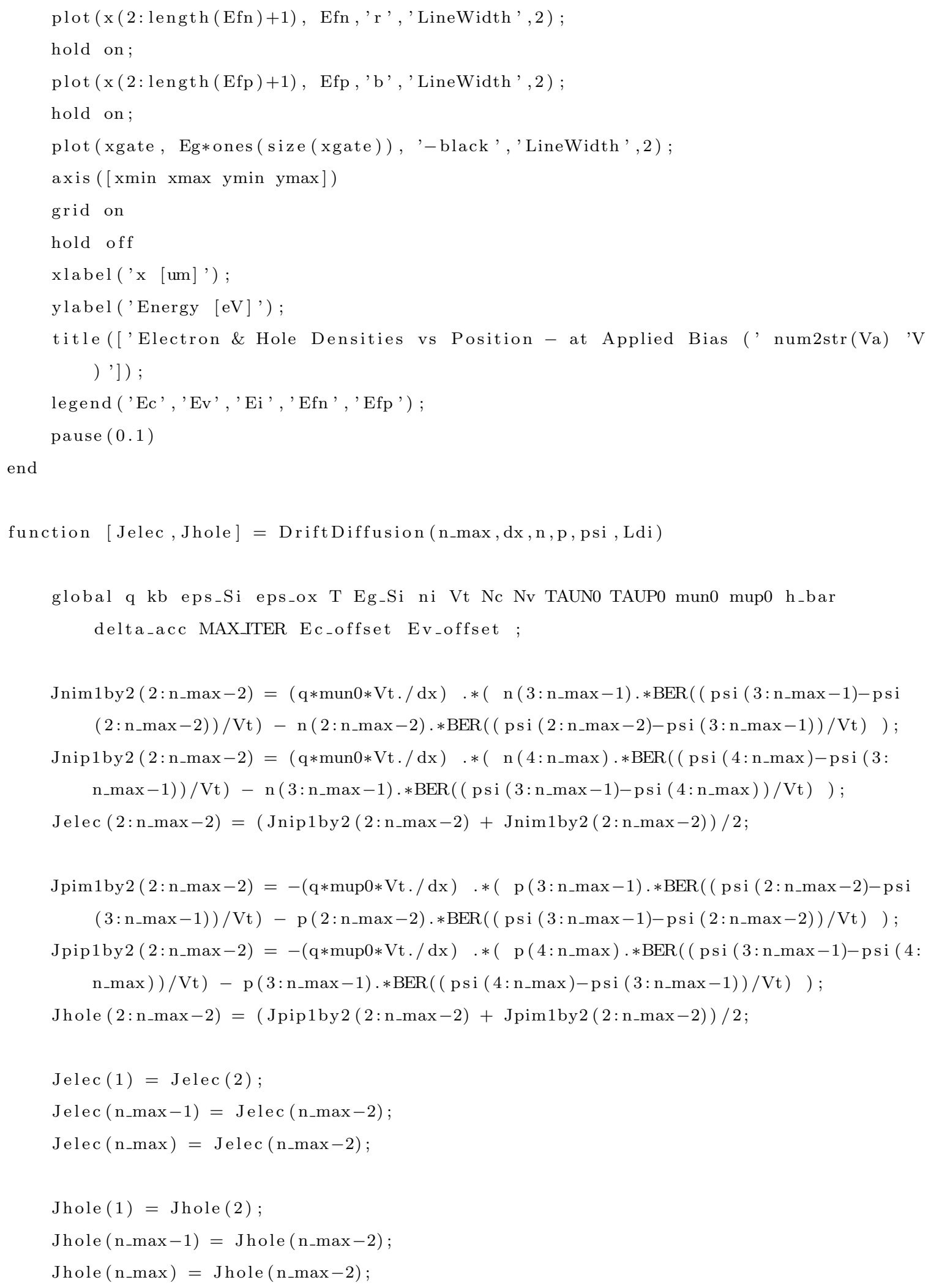




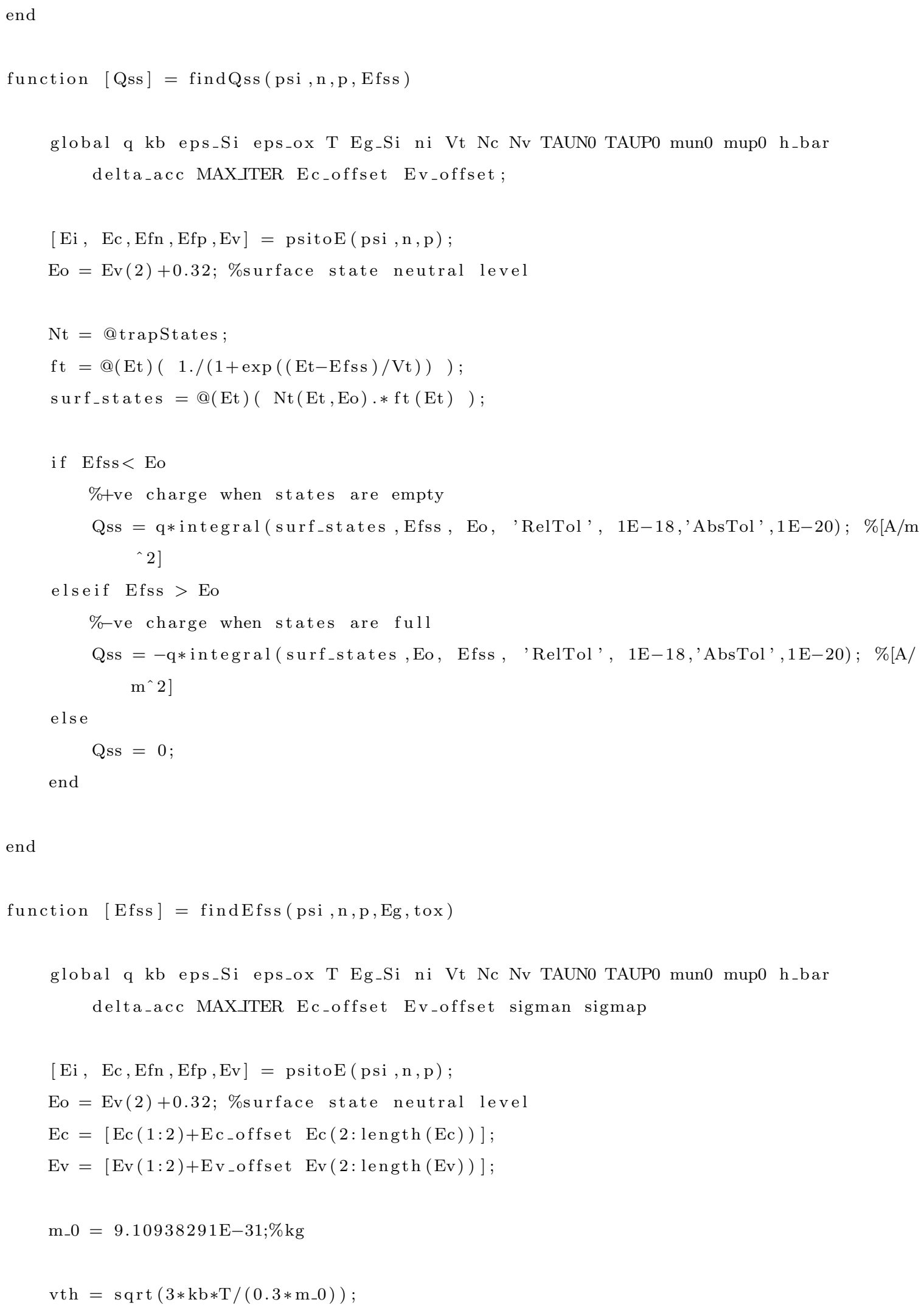




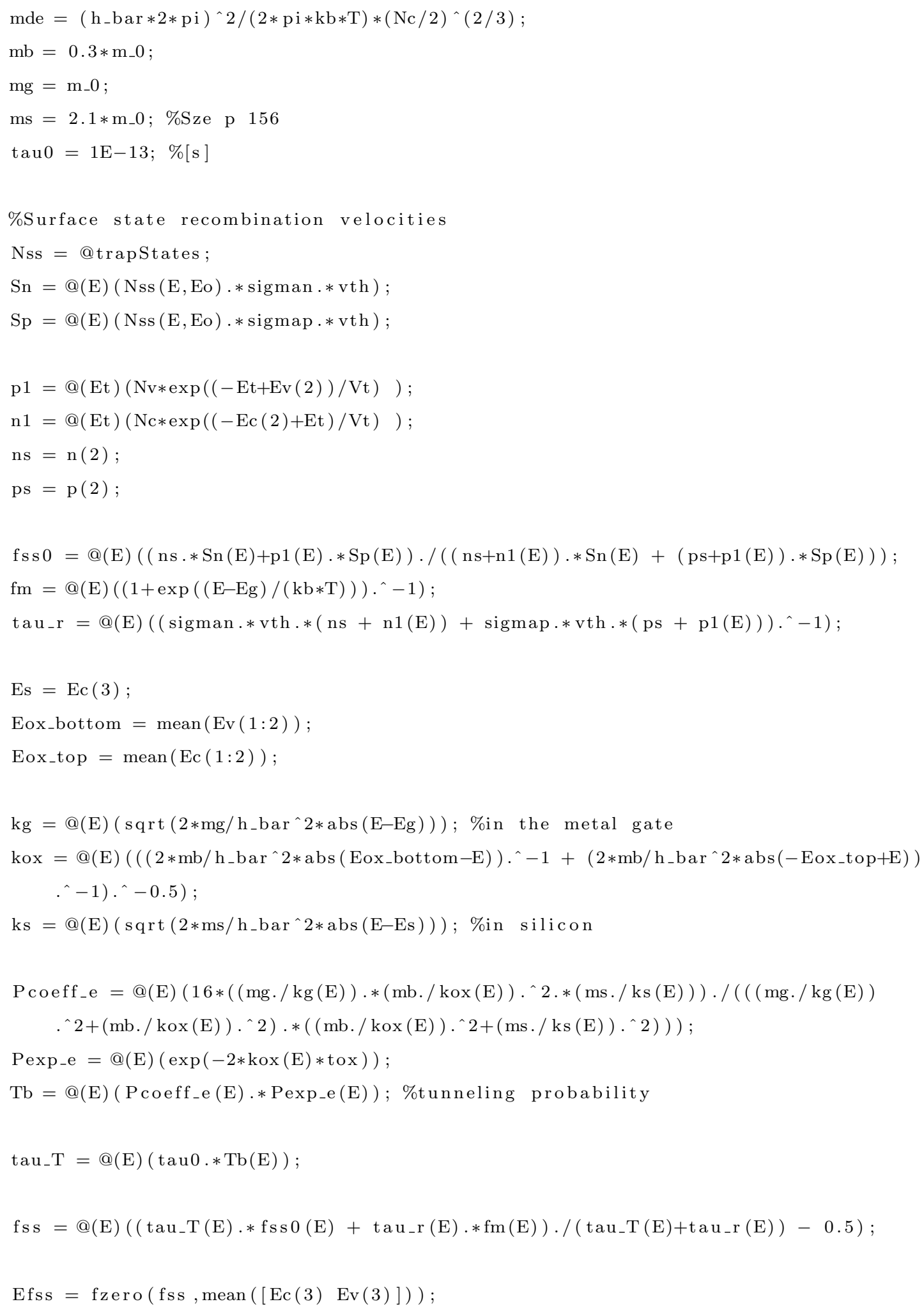




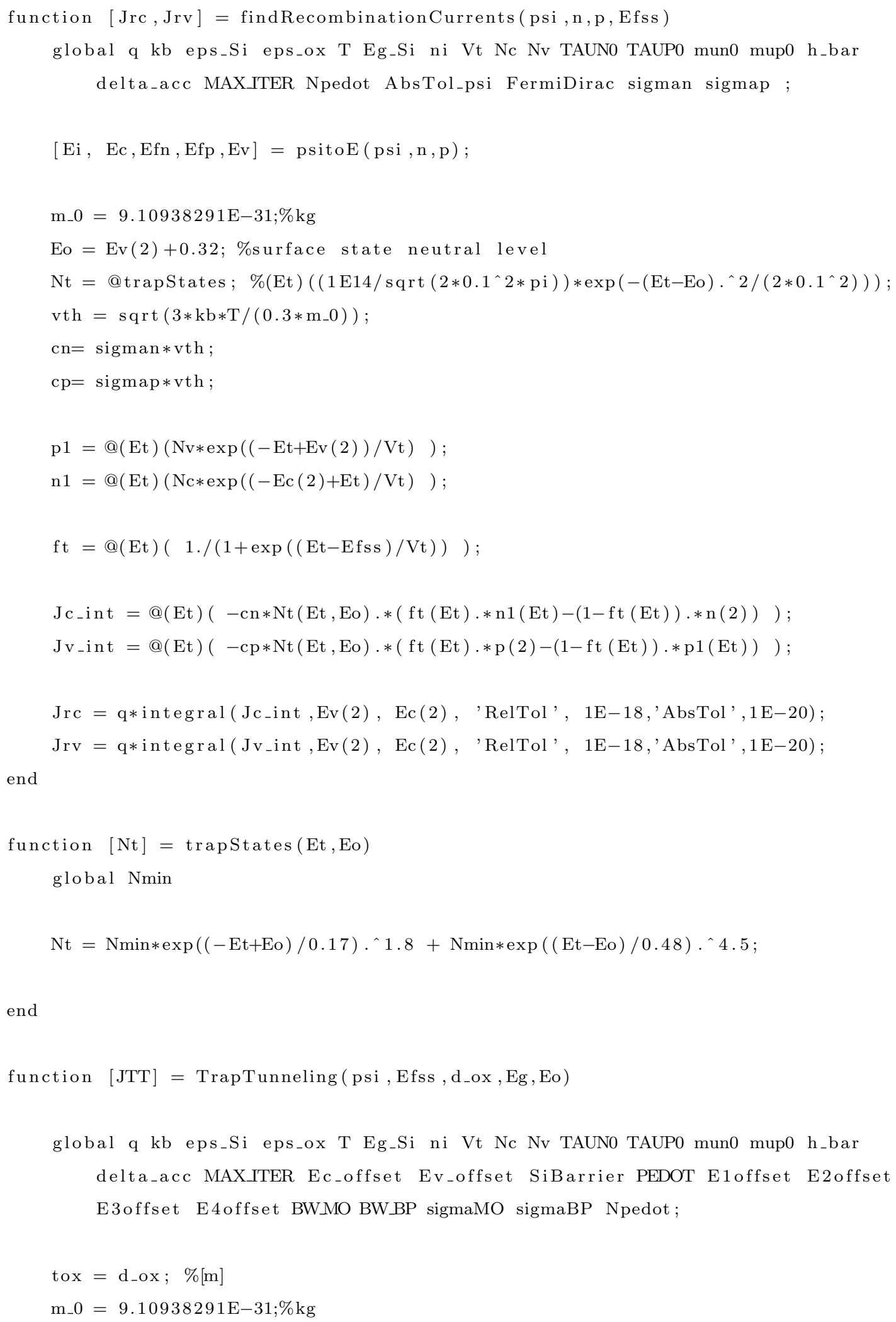




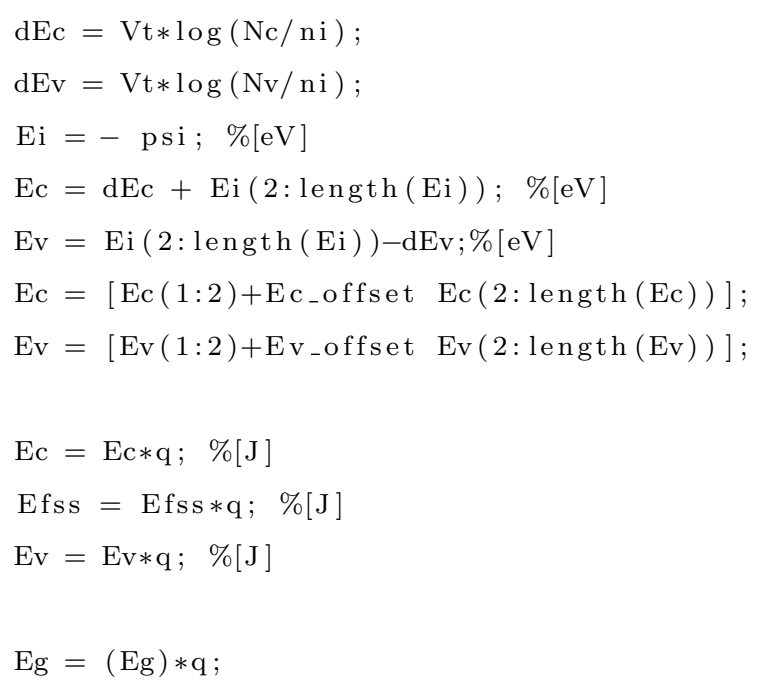

$\%$ for PEDOT

$$
\begin{aligned}
& \mathrm{E} 1=\mathrm{Eg}+\mathrm{E} 1 \text { offset } ; \\
& \mathrm{E} 2=\mathrm{Eg}+\mathrm{E} 2 \text { offset } ; \\
& \mathrm{E} 3=\mathrm{Eg}+\mathrm{E} 3 \text { offset } ; \\
& \mathrm{E} 4=\mathrm{Eg}+\mathrm{E} 4 \text { offset } ;
\end{aligned}
$$

Eox_bottom $=\operatorname{mean}(\operatorname{Ev}(1: 2))$;

Eox_top $=\operatorname{mean}(\operatorname{Ec}(1: 2))$;

\%\%\%\%\%,

\%SURFACE TRAPS\% $\%$

$\% \% \% \% \% \% \% \% \% \% \% \%$

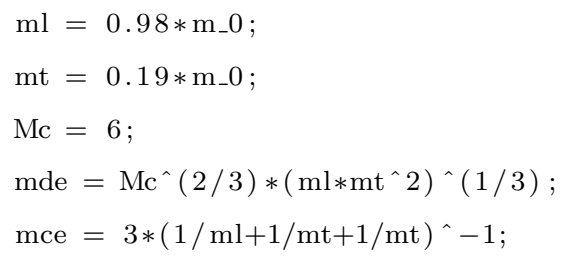




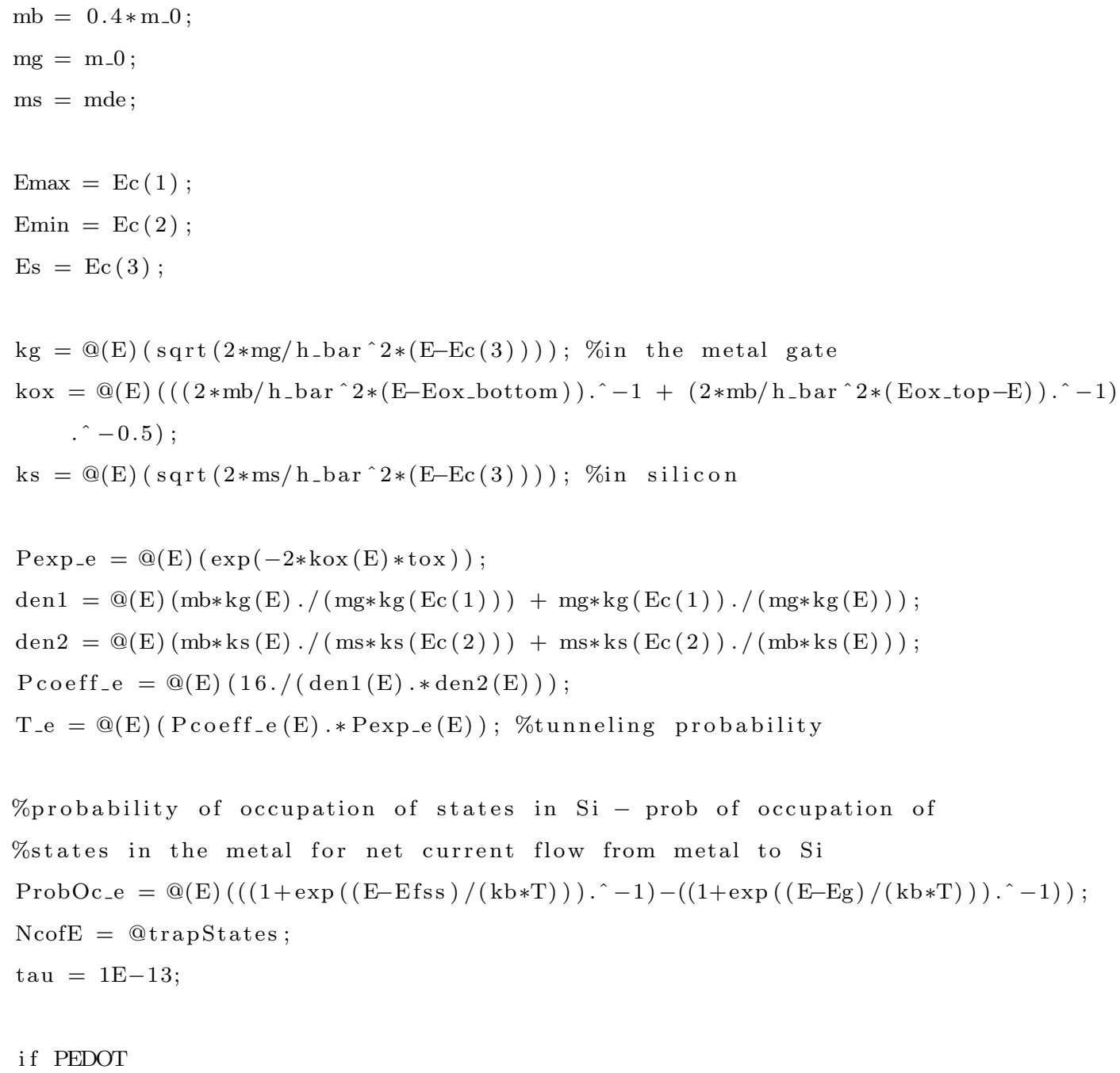




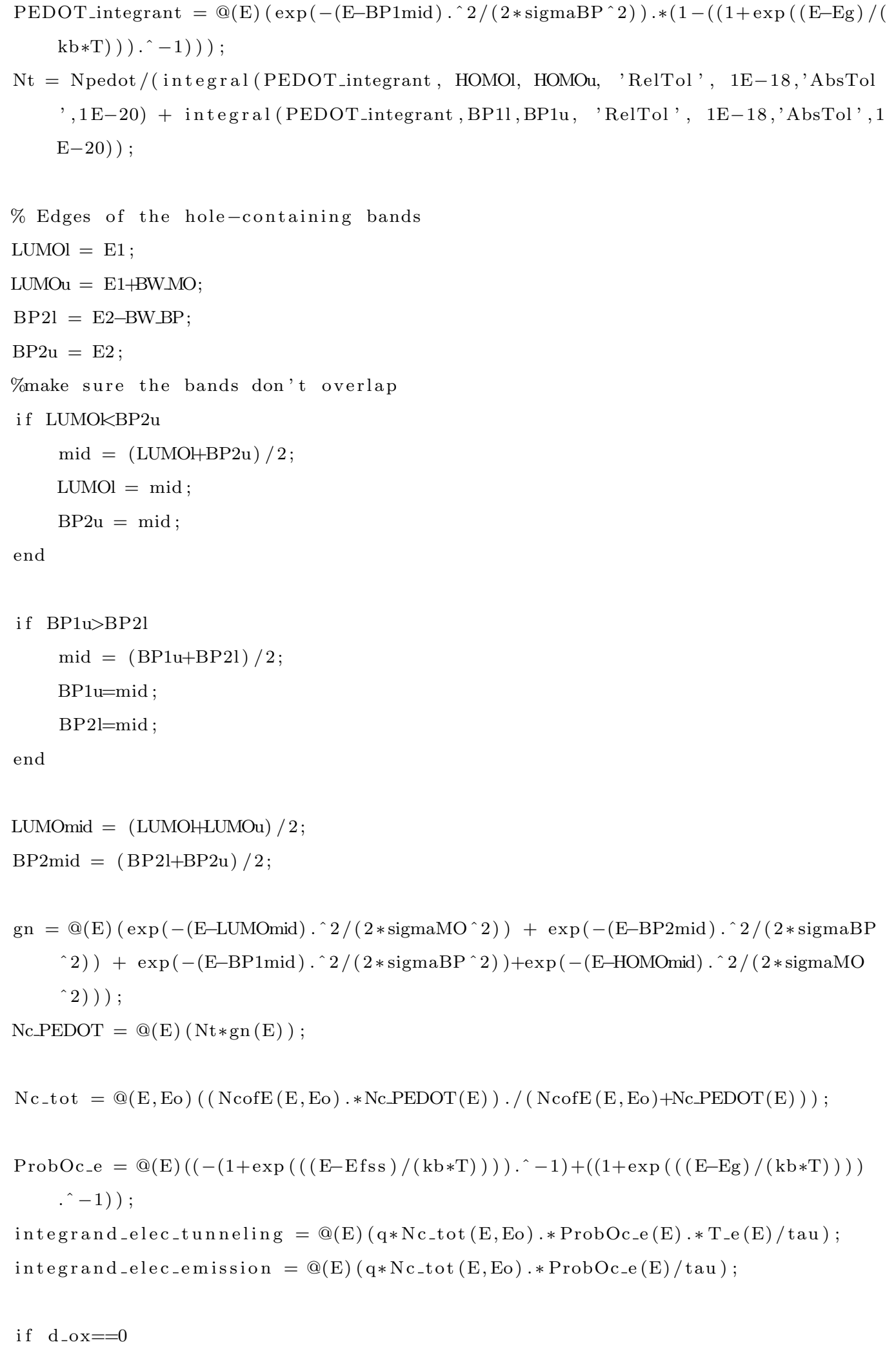


$\mathrm{JTT}=$ integral (integrand_elec_emission,Ec(3), Ec(3)+2*q, 'RelTol', $1 \mathrm{E}$ -18, 'AbsTol ', 1E-20); \%[A/m^2]

else

if HOMOl $>$ Ec(3) \% all PEDOT bands overlap with Si conduction band

JTT = integral (integrand_elec_tunneling, HOMOl, HOMOu, 'RelTol', $1 \mathrm{E}$

-18 ,'AbsTol', 1E-20) + integral (integrand_elec_tunneling, BP1l,BP1u

, 'RelTol', 1E-18,'AbsTol', 1E-20) + integral(

integrand_elec_tunneling, BP2l,BP2u, 'RelTol', 1E-18, 'AbsTol ', 1E

-20) + integral (integrand_elec_tunneling,LUMOl,LUMOu, 'RelTol' , 1

$\mathrm{E}-18$,'AbsTol' , 1E-20);

elseif HOMOu > Ec(3) \% HOMO band partially overlaps with the Si band

JTT = integral (integrand_elec_tunneling, Ec(3),HOMOu, 'RelTol', 1E

-18 ,'AbsTol', 1E-20) + integral (integrand_elec_tunneling, BP1l,BP1u

, 'RelTol', 1E-18,'AbsTol', 1E-20) + integral(

integrand_elec_tunneling, BP2l,BP2u, 'RelTol', 1E-18, 'AbsTol', $1 \mathrm{E}$

$-20)+$ integral (integrand_elec_tunneling,LUMOl,LUMOu, 'RelTol ', 1

$\mathrm{E}-18$,'AbsTol' , 1E-20);

elseif BP1l > Ec(3) \% only the HOMO band overlaps Si valence band

JTT = integral (integrand_elec_tunneling,BP1l,BP1u, 'RelTol ', 1E-18,'

AbsTol', 1E-20) + integral (integrand_elec_tunneling, BP2l,BP2u, ,

RelTol', 1E-18,'AbsTol',1E-20) + integral (

integrand_elec_tunneling, LUMOl,LUMOu, 'RelTol' , 1E-18,'AbsTol ',1E

$-20)$;

elseif BP1u > Ec(3) \% HOMO band partially overlaps with the valence band

$\mathrm{JTT}=$ integral (integrand_elec_tunneling,Ec(3),BP1u, 'RelTol ', 1E-18,'

AbsTol',1E-20) + integral (integrand_elec_tunneling, BP2l,BP2u, ,

RelTol', 1E-18,'AbsTol', 1E-20) + integral(

integrand_elec_tunneling, LUMOl,LUMOu, 'RelTol ', 1E-18,'AbsTol ',1E

$-20)$;

elseif $\mathrm{BP} 2 \mathrm{l}>\mathrm{Ec}(3)$

JTT = integral (integrand_elec_tunneling, BP2l,BP2u, 'RelTol ', 1E-18,'

AbsTol',1E-20) + integral (integrand_elec_tunneling,LUMOl,LUMOu, ,

RelTol ', 1E-18,'AbsTol' ,1E-20);

elseif $\mathrm{BP} 2 \mathrm{u}>\operatorname{Ec}(3)$

JTT = integral (integrand_elec_tunneling,Ec(3),BP2u, 'RelTol ', 1E-18,'

AbsTol',1E-20) + integral (integrand_elec_tunneling, LUMOl,LUMOu, ,

RelTol ', 1E-18,'AbsTol', 1E-20);

elseif $\mathrm{LUMOl}>\operatorname{Ec}(3)$

JTT = integral (integrand_elec_tunneling, LUMOl,LUMOu, 'RelTol', 1E

-18, 'AbsTol' , 1E-20);

els e if $\mathrm{LUMOu}>\operatorname{Ec}(3)$ 


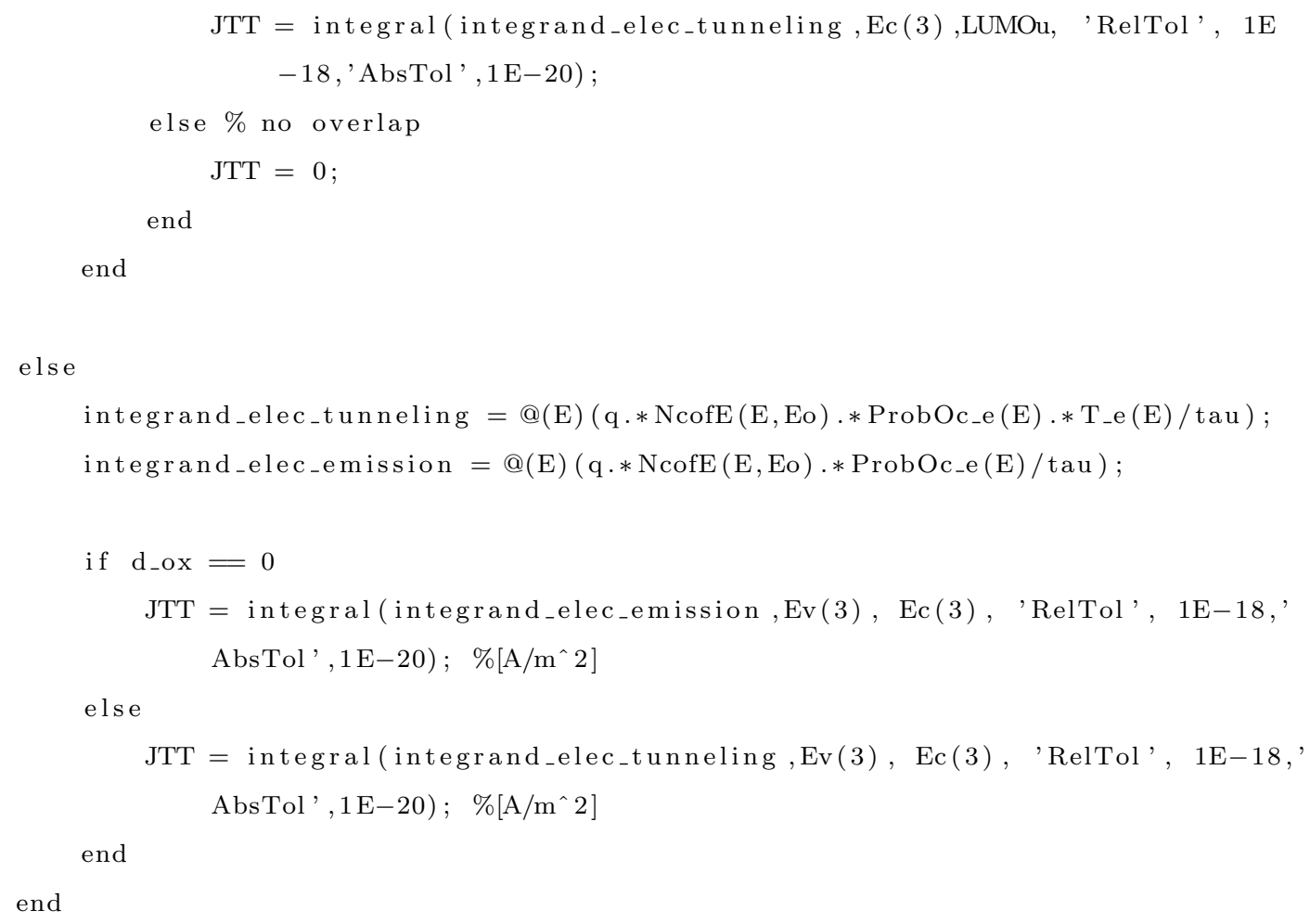

